Copyright

by

Thomas Henry Osiecki

2007 
The Dissertation Committee for Thomas Henry Osiecki certifies that this is the approved version of the following dissertation:

\section{A Search for Sterile Neutrinos in MINOS}

Committee:

Karol Lang, Supervisor

David Lambert

Sacha Kopp

Jack Ritchie

Roy Schwitters 


\title{
A Search for Sterile Neutrinos in MINOS
}

by

Thomas Henry Osiecki, B.S.

\author{
Dissertation \\ Presented to the Faculty of the Graduate School of \\ The University of Texas at Austin \\ in Partial Fulfillment \\ of the Requirements \\ for the Degree of \\ Doctor of Philosophy
}

The University of Texas at Austin

December 2007 
I dedicate this thesis to my ever-loving parents, my wonderful sister and Katie, the object of my truest affections. 


\section{Acknowledgments}

My time in graduate school has been an incredibly satisfying experience, and I have to thank my colleagues and friends that have truly helped me grow as a physicist and a person.

I first have to thank Karol Lang for literally transforming me from student to serious scientist. Few people have an advisor that really cares about framing a student's thought process in the proper fashion. Karol's focus on precisely and logically answering questions about the physical world, and especially the attention to detail have taught me things that I will carry with me in every project I undertake.

Sacha Kopp was responsible for bringing me to Texas and I thank him for having the faith to recruit me to such an incredible program. Sacha should never underestimate the impact he has had on the way I go about solving problems.

Dylan Miracle and Kevin Lee have been the greatest of friends. Our love of physics coupled with many wonderful times will always make me grateful to have met the both of you. I have no doubt that this friendship will be lifelong.

To all the members of the Neutral Current group, I thank you for the valuable experience I have gained. I especially want to thank Alfons Weber, whose logical way of thinking could not be more rational or precise, and his support throughout my tenure in the group. Brian Rebel, you are an excellent physicist and the countless hours you spent on the neutral current group working to help me graduate on time will never be forgotten. You were instrumental in my graduating. Jason Koskinen, 
I've always had fun with you and your input to the analysis helped me graduate. I cannot forget Tobi or Phil for being with me from the beginning, struggling through the many interesting problems we had to solve.

Other MINOS collaborators that I have to thank include Bob Zwaska, you are a great friend and I have learned so much about the accelerator world from you. Robert Hatcher, your patience with my software issues is unparalleled. MINOS could not run without you. Peter Shanahan, thank you for always being available and teaching me so much while I was at Fermilab. David Petyt, thank you for answering all my questions and helping me to understand the details of our analysis.

Last, my parents, sister, and Katie Hand have given me infinite support throughout this experience. I was able to conquer the hardest moments only because of these special people.

The University of Texas at Austin

December 2007 


\title{
A Search for Sterile Neutrinos in MINOS
}

\author{
Publication No.
}

Thomas Henry Osiecki, Ph.D.

The University of Texas at Austin, 2007

Supervisor: Karol Lang

MINOS, the Main Injector Neutrino Oscillation Search, is a long baseline neutrino oscillation experiment based at Fermilab National Accelerator Laboratory. The experiment uses a neutrino beam, which is measured $1 \mathrm{~km}$ downstream from its origin in the Near detector at Fermilab and then $735 \mathrm{~km}$ later in the Far detector at the Soudan mine. By comparing these two measurements, MINOS can attain a very high precision for parameters in the atmospheric sector of neutrino oscillations. In addition to precisely determining $\Delta m_{23}^{2}$ and $\theta_{23}$ through the disappearance of $\nu_{\mu}$, MINOS is able to measure $\nu_{\mu} \longrightarrow \nu_{\text {sterile }}$ by looking for a deficit in the number of neutral current interactions seen in the Far detector. In this thesis, we present the results of a search for sterile neutrinos in MINOS. 


\section{Contents}

Acknowledgments $\quad$ v

Abstract vii

$\begin{array}{lll}\text { Chapter } 1 & \text { Neutrinos } & 1\end{array}$

1.1 Introduction . . . . . . . . . . . . . . . . 1

1.2 Neutrino Oscillation Theory . . . . . . . . . . . . . . . . . . 4

1.3 Evidence for Neutrino Oscillations . . . . . . . . . . . . 8

1.3.1 Atmospheric Neutrino Anomaly . . . . . . . . . . . . . 8

1.3.2 The Solar Neutrino Deficit _. . . . . . . . . . . . 10

1.3.3 Reactor Experiments . . . . . . . . . . . . . . . . . . 14

1.3.4 Long-baseline Accelerator Experiments . . . . . . . . . . . . 16

1.3 .5 Summary . . . . . . . . . . . . . . . 20

$\begin{array}{lll}\text { Chapter } 2 & \text { Sterile Neutrinos } & 21\end{array}$

2.1 Introduction . . . . . . . . . . . . . . . . . . . . 21

2.2 Theoretical Considerations of Sterile Neutrinos . . . . . . . . . . . 21

2.3 Experimental Limits on the Existence of Sterile Neutrinos . . . . . . 23

2.4 Sterile Neutrinos in Astrophysics and Cosmology . . . . . . . . . . . 32

2.5 Searching for Sterile Neutrinos in MINOS . . . . . . . . . . . 35 
3.1 Overview . . . . . . . . . . . . . . . . . 37

3.2 Neutrino Beam . . . . . . . . . . . . . . . . . . . . . . . . 38

3.3 The MINOS Detectors . . . . . . . . . . . . . . . . . . . 42

3.3.1 Particle Detection . . . . . . . . . . . . . . . . 42

$3.3 .2 \quad$ Scintillator . . . . . . . . . . . . . . . 43

3.3.3 Photomultiplier Tubes . . . . . . . . . . . . . . . . 44

3.3.4 Steel and Magnetic Field . . . . . . . . . . . . . 45

3.4 The MINOS Spectrometers . . . . . . . . . . . . . . . . . 45

3.4 .1 Near Detector . . . . . . . . . . . . . . . 45

3.4 .2 Far Detector . . . . . . . . . . . . . . . 49

3.4 .3 Calibration Detector . . . . . . . . . . . . . 51

3.5 Light Injection System . . . . . . . . . . . . . . . . . . 52

3.6 Summary . . . . . . . . . . . . . . . . . . . . 52

$\begin{array}{lll}\text { Chapter } 4 & \text { Event Reconstruction } & 53\end{array}$

4.1 MINOS Monte Carlo . . . . . . . . . . . . . . . . . . . . . 53

4.2 Reconstruction Software . . . . . . . . . . . . . . . 54

4.2.1 First Stage of Signal Processing . . . . . . . . . . . . . 54

4.2.2 Second Stage of Signal Processing _ . . . . . . . . . . . . 54

4.2 .3 Event Isolation . . . . . . . . . . . . . . . . . . . . 55

4.2 .4 Cluster Formation . . . . . . . . . . . . . . . . 67

4.2 .5 Shower Formation . . . . . . . . . . . . . . 67

4.2 .6 Track Formation . . . . . . . . . . . . . 67

4.2 .7 Track Fitting . . . . . . . . . . . . . . . 68

4.2 .8 Event Formation . . . . . . . . . . . . . . . . . . 68

4.3 Summary . . . . . . . . . . . . . . . . . . 68 
5.1 Selecting Quality Beam . . . . . . . . . . . . 70

5.2 Fiducial Volume Containment . . . . . . . . . . . . . . . . 72

5.3 Data Cleaning . . . . . . . . . . . . . . . . 73

5.4 Charged Current Event Selection _. . . . . . . . . . . . 76

5.5 Neutrino Flux Tuning . . . . . . . . . . . . . . . . . . . 77

5.6 Neutral Current Selection Variables . . . . . . . . . . . . . . . . . . 81

5.7 Near Detector Charged Current and Neutral Current Energy Spectra 86

5.8 Summary . . . . . . . . . . . . . . . . . . . . . . . . 92

$\begin{array}{lll}\text { Chapter } 6 & \text { Reconstruction Validation Studies } & 93\end{array}$

6.1 Introduction . . . . . . . . . . . . . . . . . . . . 93

6.2 Detector Position Studies _. . . . . . . . . . . . . . . . . 94

6.3 Intensity Studies . . . . . . . . . . . . . . . . . 109

6.4 Batch Studies . . . . . . . . . . . . . . . 136

6.5 Conclusions . . . . . . . . . . . . . . . . . . . . . . . 141

Chapter 7 NuMI Beam Neutrinos in the Far Detector 142

7.1 Blinding Procedure . . . . . . . . . . . . . . . . . 143

7.2 Main Sources of Background _. . . . . . . . . . . . . . 143

7.3 Fiducial Volume Containment . . . . . . . . . . . . . . . . . . . 144

7.4 Data Cleaning . . . . . . . . . . . . . . . . . 146

7.4.1 Removal of Light Injection Events . . . . . . . . . . . . 146

7.4 .2 Singles Noise Removal . . . . . . . . . . . . . . . . . . 147

7.4.3 Removal of Cosmic Ray Muons . . . . . . . . . . . . . . . . . 148

7.4 .4 Event Timing . . . . . . . . . . . . . . . . . . . . . . . 149

7.5 Effects of Data Cleaning Criteria . . . . . . . . . . . . 150

7.5.1 Beam Neutrino Losses . . . . . . . . . . . . . . . . . . . 150 
7.5.2 Efficiency of Data Cleaning . . . . . . . . . . . . . . . 150

7.6 Energy Spectra in the Far Detector . . . . . . . . . . . . . . . 152

7.7 Summary . . . . . . . . . . . . . . . . . . 153

$\begin{array}{lll}\text { Chapter } 8 & \text { Extrapolation from the Near to Far Detector } & 154\end{array}$

8.1 Marquardt-Style Minimization . . . . . . . . . . . . . . 155

8.2 Systematic Parameters used in the NDFit . . . . . . . . . . . 157

8.2 .1 Beam Systematics . . . . . . . . . . . . . . . . 158

8.2 .2 Cross-section Systematics . . . . . . . . . . . . . 159

8.2 .3 Reconstruction Systematics . . . . . . . . . . . . . . . 161

8.2.4 Parameters used in Near detector Fit . . . . . . . . . . . 162

8.3 Systematic Studies . . . . . . . . . . . . . . . . . . 168

8.3 .1 Systematic Study I . . . . . . . . . . . . . . . 173

8.3 .2 Systematic Study II . . . . . . . . . . . . . . . 177

8.3 .3 Systematic Study III . . . . . . . . . . . . . . . . . . . 180

8.3 .4 Systematic Study IV . . . . . . . . . . . . . . . . . . 184

8.3.5 Systematic Study V . . . . . . . . . . . . . . . . 188

8.4 Summary of Systematic Studies . . . . . . . . . . . . . . . . . . 191

8.5 Mock Data Challenge . . . . . . . . . . . . . . . . . . . 191

8.5.1 Mock Data Challenge Set I Results . . . . . . . . . . . . . . . 192

8.5.2 Mock Data Challenge Set II Results . . . . . . . . . . . . 196

8.6 Conclusions . . . . . . . . . . . . . . . . . . . 200

$\begin{array}{lll}\text { Chapter } 9 & \nu_{\mu} \rightarrow \nu_{\text {sterile }} \text { Oscillation Search } & 201\end{array}$

9.1 Introduction . . . . . . . . . . . . . . . . . . . . . . . 201

9.2 Data Set and Cross-checks . . . . . . . . . . . . . . . . . . 201

9.2.1 Quantities as a function of time ............ 203

9.2 .2 Timing ......................... 206 
9.2.3 Fiducial Volume Distributions . . . . . . . . . . . . . . . 207

9.3 Near Detector Fit and Far Detector Prediction . . . . . . . . . . . 210

9.4 Measuring the sterile fraction . . . . . . . . . . . . . . 215

9.5 Conclusions . . . . . . . . . . . . . . . . . . . . 221

Appendix A Example of Slicing Algorithm 222

A.1 Conclusions . . . . . . . . . . . . . . . . . . . . . 224

$\begin{array}{lll}\text { Appendix B Systematic Parameters used in NDFit } & 234\end{array}$

Appendix C Far Detector Energy Spectra Composition 245

$\begin{array}{lr}\text { Bibliography } & 245\end{array}$

$\begin{array}{lr}\text { Bibliography } & 252\end{array}$

$\begin{array}{ll}\text { Vita } & 262\end{array}$ 


\section{Chapter 1}

\section{Neutrinos}

\subsection{Introduction}

The neutrino was first proposed in 1930 by Wolfgang Pauli [1] to explain an anomaly observed in $\beta$ decay. At the time $\beta$ decay was believed to be a two body problem and only an electron (or positron) should be emitted. Through kinematics, one expected a discrete energy for the electron, but what experimentalists observed was that the emitted electron had a continuous energy spectrum associated with it. It seemed that conservation of energy was violated. To remedy this situation Pauli proposed a hypothetical third particle which was neutral, of very light mass, spin $1 / 2$ and had a low interaction rate in order to save energy conservation.

Enrico Fermi coined the term "neutrino" and incorporated it into his theory of nuclear $\beta$ decay [2]. The success of Fermi's theory gave solid theoretical credence to the neutrino, but it was not actually discovered until 1956 by Reines and Cowan at Savannah River Plant in Augusta, Georgia [3]. The neutrino that they discovered was the electron neutrino, $\bar{\nu}_{e}$, and it is now known that there exist three flavors of neutrinos, each having a partner with one of the three known leptons: e, $\mu$, and $\tau$. In addition to this, LEP has measured the width of the $\mathrm{Z}$ Boson to be consistent 
Table 1.1: Current knowledge of the neutrino flavor eigenstate masses

\begin{tabular}{|c|c|}
\hline Neutrino Flavor & Mass \\
\hline$\nu_{e}$ & $<2 \mathrm{eV}^{2} / \mathrm{c}^{4}$ \\
\hline$\nu_{\mu}$ & $<0.19 \mathrm{MeV}^{2} / \mathrm{c}^{4}$ \\
\hline$\nu_{\tau}$ & $<18.2 \mathrm{MeV}^{2} / \mathrm{c}^{4}$ \\
\hline
\end{tabular}

with the existence of three neutrino flavors whose masses are less than half the mass of the Z Boson [4]. The exact result for the number of neutrino flavors from LEP is $2.984 \pm 0.008$. The muon neutrino was first observed in 1962 at Brookhaven National Laboratory [5] by the collaboration of Melvin Schwartz, Leon Lederman and Jack Steinberger. The tau neutrino was observed by the DONUT collaboration in 2000 [6] at Fermi National Accelerator Laboratory (Fermilab). At present only experimental limits exist on the masses of neutrino flavor eigenstates, which are summarized in Table 1.1 [7].

In the Standard Model of particle physics neutrinos interact solely via the weak force and are assumed to be massless. Two separate modes of interaction exist for the neutrino. The neutral current interaction is:

$$
\nu_{l}+N \rightarrow \nu_{l}+N^{\prime} \quad l=e, \mu, \tau
$$

where a neutrino $\nu_{l}$ interacts with the particle $\mathrm{N}$, the neutrino looses some energy but continues to propagate afterwards, and $\mathrm{N}^{\prime}$ is the resulting hadronic system. It should be noted that one cannot determine the flavor of the neutrino in a neutral current interaction. The second interaction is the charged current:

$$
\nu_{l}+N \rightarrow l^{-}+N^{\prime} \quad l=e, \mu, \tau
$$

where a neutrino interacts with a particle $\mathrm{N}$ and a charged lepton that corresponds to the neutrino's flavor is produced. As before, $\mathrm{N}^{\prime}$ is the hadronic system from the 


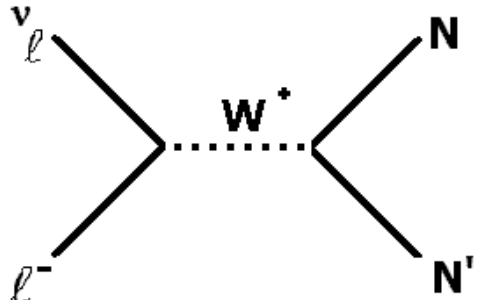

Charged Current $\ell=\mathbf{e}, \mu, \tau$

(a) $\mathrm{CC}$

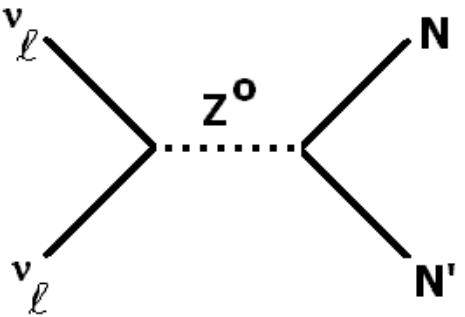

Neutral Current

$\ell=\mathbf{e}, \mu, \tau$

(b) $\mathrm{NC}$

Figure 1.1: Feynman diagrams for charged current and neutral current neutrino interactions.

interaction. In this particular case, the neutrino deposits all of its energy and the original flavor can be identified through the lepton. Both of these processes are shown via Feynman diagrams in Figure 1.1.

In the past decade compelling evidence for neutrino flavor oscillations has been accepted by the particle physics community. It was Pontecorvo who in 1957 first suggested neutrino and anti-neutrino oscillations [14] which later evolved into a theory in which neutrinos with non-zero mass could oscillate between flavors [15]. It was the Super-Kamiokande experiment [21] that first discovered neutrino oscillations through their result showing a deficit in the number of muon neutrinos coming from atmospheric neutrino interactions as a function of zenith angle. This is known as the Atmospheric Neutrino Anomaly. The Sudbury Neutrino Observatory (SNO) made another confirmation for neutrino oscillations, when they solved the Solar Neutrino Deficit problem [22] introduced by Ray Davis' [16] experiment by measuring all three types of neutrinos from the sun via their neutral current interactions. It is now accepted irrefutably that neutrinos do indeed oscillate.

In the following sections of this chapter, we briefly describe the theoretical basis of neutrino oscillations and the experimental evidence associated with the 
phenomena. In the second chapter we elaborate more on the hypothetical sterile neutrino for which we search.

\subsection{Neutrino Oscillation Theory}

Neutrinos are detected in their weak flavor eigenstates. This does not imply that they are necessarily parallel to their mass eigenstates, and so we can write the weak flavor eigenstates as a linear combination of mass eigenstates. Note that a consequence of neutrino oscillations is a non-zero neutrino mass. One can take this into consideration by writing

$$
\left|\nu_{\alpha}\right\rangle=\sum_{i} U_{\alpha i}\left|\nu_{i}\right\rangle
$$

where $\left|\nu_{\alpha}\right\rangle$ is a neutrino with definite flavor, $\alpha=e, \mu, \tau$, and $\left|\nu_{i}\right\rangle$ is a neutrino mass eigenstate with $\mathrm{i}=1,2,3 . \quad U_{\alpha i}$ represents the Pontecorvo-Maki-Nakagawa-Sakata matrix [32], which is also referred to as the PMNS matrix. Note that the PMNS matrix is $n \times n$ where $n$ is the number of neutrino flavors. The matrix elements for three neutrinos are defined as

$$
U=\left(\begin{array}{ccc}
U_{e 1} & U_{e 2} & U_{e 3} \\
U_{\mu 1} & U_{\mu 2} & U_{\mu 3} \\
U_{\tau 1} & U_{\tau 2} & U_{\tau 3}
\end{array}\right)
$$

which has the form

$$
U=\left(\begin{array}{ccc}
c_{12} c_{13} & s_{12} c_{13} & s_{13} e^{-i \delta} \\
-s_{12} c_{23}-c_{12} s_{23} s_{13} e^{i \delta} & c_{12} c_{23}-s_{12} s_{23} s_{13} e^{i \delta} & s_{23} c_{13} \\
s_{12} s_{23}-c_{12} c_{23} s_{13} e^{i \delta} & -c_{12} s_{23}-s_{12} c_{23} s_{13} e^{i \delta} & c_{23} s_{13}
\end{array}\right)
$$


where $s_{x y}=\sin \theta_{x y}$ and $c_{x y}=\cos \theta_{x y}$, and the phase $\delta$ is non-zero only if neutrino oscillations violate $\mathrm{CP}$ symmetry. This matrix can be expanded into $U=$ $U_{\text {atmospheric }} \times U_{\nu_{e} \text { appearance }} \times U_{\text {solar }}$, such that

$$
U=\left(\begin{array}{ccc}
1 & 0 & 0 \\
0 & c_{23} & s_{23} \\
0 & -s_{23} & c_{23}
\end{array}\right) \times\left(\begin{array}{ccc}
c_{13} & 0 & s_{13} e^{-i \delta} \\
0 & 1 & 0 \\
-s_{13} e^{i \delta} & 0 & c_{13}
\end{array}\right) \times\left(\begin{array}{ccc}
c_{12} & s_{12} & 0 \\
-s_{12} & c_{12} & 0 \\
0 & 0 & 1
\end{array}\right)
$$

Equation 1.3 is expanded as a product of three matrices for physical reasons. Each of the three matrices can be associated with experimental searches for neutrino oscillations. The first matrix depends on $\theta_{23}$, which is the angle measured by atmospheric and long baseline neutrino experiments. This includes experiments such as Super-Kamiokande and MINOS. The second matrix depends on $\theta_{13}$, which the Chooz experiment has measured to be very small, meaning that this term is approximately the identity matrix. The third matrix depends on $\theta_{12}$, which can be measured by solar neutrino experiments and long baseline reactor experiments such as SNO and KamLAND. An interesting consequence of the middle term being close to the identity matrix is that the first and third terms are de-coupled from each other and it is possible to analyze their results using only a two neutrino model. We discuss this more later.

It is not currently known whether neutrinos are their own anti-particles, otherwise defined as a Majorana particle. If this happens to be the case, then the PMNS matrix needs to be multiplied by another matrix given by

$$
\left(\begin{array}{ccc}
e^{i \alpha_{1} / 2} & 0 & 0 \\
0 & e^{i \alpha_{2} / 2} & 0 \\
0 & 0 & 1
\end{array}\right)
$$

The phases $\alpha_{1}, \alpha_{2}$ are non-zero only if neutrinos are Majorana, otherwise it reduces 
to the identity matrix.

Since $\left|\nu_{i}\right\rangle$ are mass eigenstates, from quantum mechanics one can describe their propagation by plane wave solutions of the Schrödinger equation:

$$
\left|\nu_{i}(t)\right\rangle=e^{-i\left(E_{i} t-\overrightarrow{p_{i}} \vec{x}\right)}\left|\nu_{i}(0)\right\rangle
$$

where $c=\hbar=1, E_{i}$ is the energy of the mass eigenstate $\mathrm{i}, \mathrm{t}$ is the time from the start of the propagation, $\overrightarrow{p_{i}}$ is the three dimensional momentum and $\vec{x}$ is the current position of the particle relative to its starting position.

In the ultra relativistic limit $\left|\vec{p}_{i}\right|=p_{i}>>m_{i}$, and assuming all the $\left|\nu_{i}\right\rangle$ components have a common energy E, one can use Taylor expansions to approximate

$$
E=\sqrt{p_{i}^{2}+m_{i}^{2}} \approx p_{i}+\frac{m_{i}^{2}}{2 p_{i}} \approx E+\frac{m_{i}^{2}}{2 E}
$$

This approximation is valid for practically all experiments. The Lorentz factor $(\gamma$ $\left.=\frac{E}{m}\right)$ is usually greater than $10^{6}$, which justifies our relativistic approximation. We can use this approximation and the fact that $c t \approx L$ and substitute into Equation 1.5 which then becomes

$$
\left|\nu_{i}(L)\right\rangle=e^{i m_{i}^{2} L / 2 E}\left|\nu_{i}(0)\right\rangle
$$

A more rigorous treatment of neutrinos as wave packets and hence avoiding the assumption of a common energy $\mathrm{E}$ for all $\left|\nu_{i}\right\rangle$ leads to the same result [33].

Since each mass eigenstate will have a different mass, they each propagate at different speeds. And since we assumed that the mass eigenstates are combinations of flavor eigenstates, the speed differences cause interference between flavor components. This means that it is possible to observe a neutrino with a different flavor than it was originally created with. The probability that a neutrino of flavor $\alpha$ will later be observed having flavor $\beta$ is 


$$
P_{\alpha \rightarrow \beta}=\left|\left\langle\nu_{\beta} \mid \nu_{\alpha}(t)\right\rangle\right|^{2}=\left|\sum_{i} U_{\alpha i}^{*} U_{\beta i} e^{i m_{i}^{2} L / 2 E}\right|^{2}
$$

which can be expanded to read

$$
\begin{aligned}
P_{\alpha \rightarrow \beta}=\delta_{\alpha \beta} & -4 \sum_{i>j} \operatorname{Re}\left(U_{\alpha i}^{*} U_{\beta i} U_{\alpha j} U_{\beta j}^{*}\right) \sin ^{2}\left(\frac{\Delta m_{i j}^{2} L}{4 E}\right) \\
& +2 \sum_{i>j} \operatorname{Im}\left(U_{\alpha i}^{*} U_{\beta i} U_{\alpha j} U_{\beta j}^{*}\right) \sin ^{2}\left(\frac{\Delta m_{i j}^{2} L}{4 E}\right)
\end{aligned}
$$

where $\Delta m_{i j}^{2}=m_{i}^{2}-m_{j}^{2}$.

Recent neutrino oscillation data implies that two of the neutrino mass eigenstates are degenerate in comparison with the third. In a three neutrino model, the differences in mass squared can be related by $\Delta m_{12}^{2}+\Delta m_{23}^{2}=\Delta m_{13}^{2}$. The full hierarchy of the masses $m_{1}, m_{2}$ and $m_{3}$ is presently not known since the sign of $\Delta m^{2}$ has not yet been determined. As will be discussed in the following section, several experiments have made measurements of $\Delta m^{2}$. Super-Kamiokande [21] and SNO [22] have measured two different $\Delta m^{2}$ 's. SNO gave a result with $\left|\Delta m_{12}^{2}\right| \approx 8 \times 10^{-5} \mathrm{eV}^{2}$ and Super-Kamiokande gave a result of $\left|\Delta m_{23}^{2}\right| \approx 2 \times 10^{-3} \mathrm{eV}^{2}$. We can choose the separation between $m_{1}$ and $m_{2}$ to be the solar neutrino measurement from SNO. Hence,

$$
\left|\Delta m_{12}^{2}\right| \ll\left|\Delta m_{13}^{2}\right| \approx\left|\Delta m_{23}^{2}\right|
$$

The Chooz result [27] suggests that $\theta_{13}$ is quite small and so the atmospheric $\left(\Delta m_{23}^{2}=\Delta m_{\text {atm }}^{2}\right)$ and the solar $\left(\Delta m_{12}^{2}=\Delta m_{\text {sol }}^{2}\right)$ difference in mass squared are de-coupled from each other. Therefore instead of using the general oscillation probability Equation 1.6, one can simply use a two neutrino oscillation approach to analyze most experiments. 
In a two neutrino approach the original PMNS mixing matrix reduces to

$$
U=\left(\begin{array}{cc}
\cos \theta & \sin \theta \\
-\sin \theta & \cos \theta
\end{array}\right)
$$

and following precisely the same arguments outlined above, we find that the oscillation probability becomes

$$
P_{\alpha \rightarrow \beta, \alpha \neq \beta}=\sin ^{2} 2 \theta \sin ^{2}\left(1.27 \frac{\Delta m^{2} L}{E_{\nu}}\left[\frac{e V^{2} k m}{G e V}\right]\right)
$$

where the factor of 1.27 comes from our choice of unit inputs to Equation 1.8, which are chosen since typical neutrino energies in MINOS are in the GeV range and the distance the neutrinos travel is $735 \mathrm{~km}$.

\subsection{Evidence for Neutrino Oscillations}

\subsubsection{Atmospheric Neutrino Anomaly}

Atmospheric neutrinos are produced in the decay of pions and kaons created by primary cosmic ray interactions in the upper atmosphere. The typical reaction is shown pictorially in Figure 1.2 and the following formulas.

$$
\begin{array}{r}
p+N \rightarrow \pi^{ \pm}+X \\
\pi^{ \pm} \rightarrow \mu^{ \pm}+\nu_{\mu}\left(\bar{\nu}_{\mu}\right) \\
\mu^{ \pm} \rightarrow e^{ \pm}+\nu_{e}\left(\bar{\nu}_{e}\right)+\nu_{\mu}\left(\bar{\nu}_{\mu}\right)
\end{array}
$$

As shown by the above reactions, the approximate ratio of $\nu_{\mu} / \nu_{e} \approx 2$. The SuperKamiokande experiment is a detector located $1000 \mathrm{~m}$ underground in the Mozumi Mine, consisting of 50000 tons of pure water surrounded by 11200 photomultiplier 


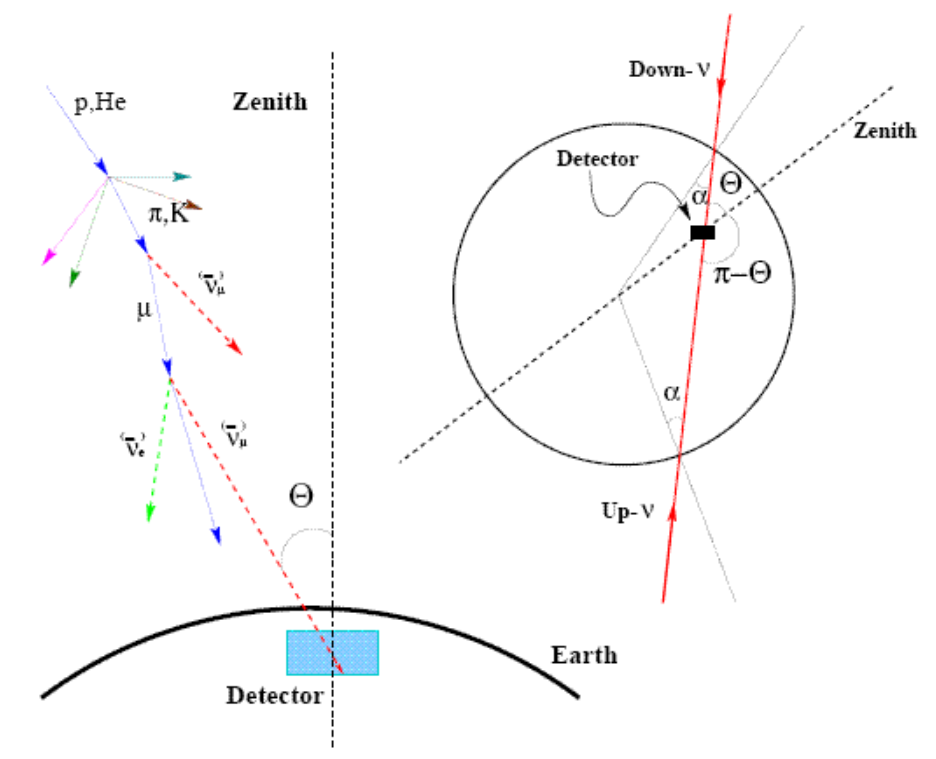

Figure 1.2: The image represents the typical scheme that creates atmospheric neutrinos. A proton or some heavier ion will interaction in the upper atmosphere of the Earth and a shower of particles will eventually decay into neutrinos which interact in a detector given by the box in the left figure. The right image is a more detailed picture of the Earth and how neutrinos can interact from any direction in the detector.

tubes. It is a cylindrical structure that is $41.4 \mathrm{~m}$ tall and $39.3 \mathrm{~m}$ across and it detects neutrino interactions through the production of Cherenkov radiation. The pattern of hits resulting from the Cherenkov cone provides information on the direction and flavor of the neutrino. Super-Kamiokande measured the ratio of $\nu_{\mu} / \nu_{e}$ and found a deficit compared to the expected value of $\approx 2$. This result was the discovery of neutrino oscillations [21]. The experiment found that this deficit had an interesting correlation with the zenith angle of the neutrino interaction. It appeared that the deficit occurred for upward going neutrinos that had to travel through the opposite side of the Earth to enter the bottom of the Super-Kamiokande detector. Muon neutrinos that had a longer distance to travel seemed to be disappearing. The data 

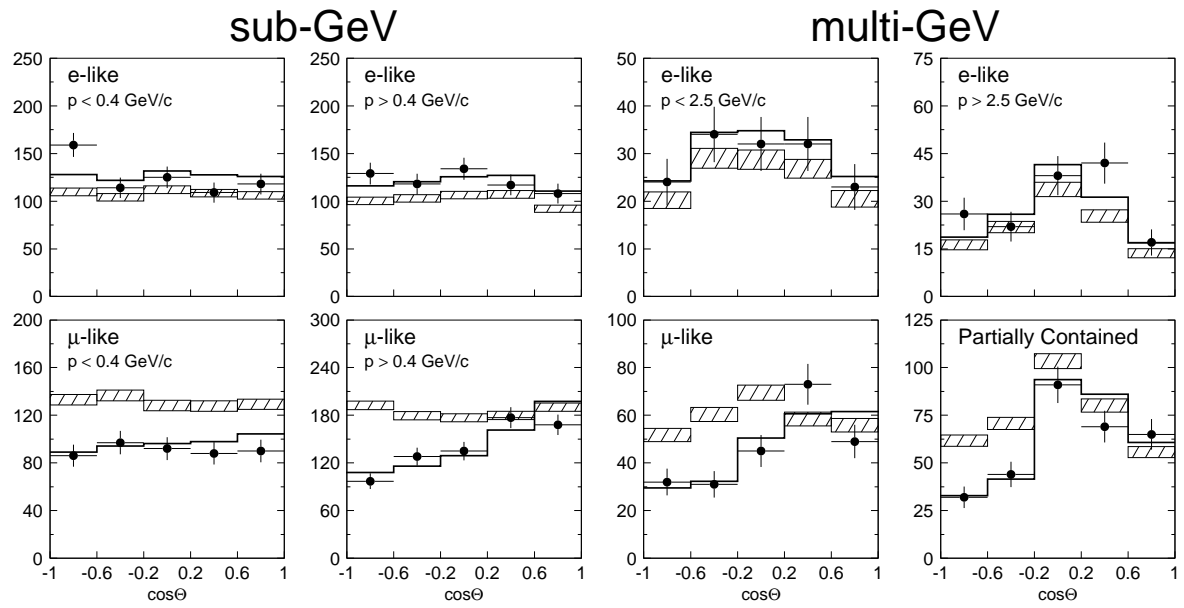

Figure 1.3: Zenith angle distributions of $\mu$-like and $e$-like events for sub-GeV and multi-GeV data sets. Upward-going particles have $\cos \Theta<0$ and downward-going particles have $\cos \Theta>0$. Sub-GeV data are shown separately for $p<400 \mathrm{MeV} / c$ and $p>400 \mathrm{MeV} / c$. Multi-GeV e-like distributions are shown for $p<2.5 \mathrm{GeV} / c$ and $p>2.5 \mathrm{GeV} / c$ and the multi-GeV $\mu$-like are shown separately for $\mathrm{FC}$ and $\mathrm{PC}$ events. The hatched region shows the Monte Carlo expectation for no oscillations normalized to the data live-time with statistical errors. The bold line is the best-fit expectation for $\nu_{\mu} \leftrightarrow \nu_{\tau}$ oscillations with the overall flux normalization fitted as a free parameter [21].

was analyzed with a neutrino oscillation hypothesis and the deficit results are shown in Figure 1.3.

\subsubsection{The Solar Neutrino Deficit}

It was many decades before physicists began to accept that neutrino oscillations occur in nature. The first mystery leading to acceptance was the Solar Neutrino Deficit. Although much work has been done to understand the inner structure of the sun by people like Lane [8], von Weizsacker [9], Gamow [10], Bethe [11], and Oke [12] it was John Bahcall that contributed unique predictions for the Standard Solar Model [13]. This model is the currently accepted theory about the processes 
within the sun. According to this theory, the dominant fusion process occurring in the sun is

$$
4 p \rightarrow^{4} \mathrm{He}+2 \nu_{e}+2 e^{+}
$$

which occurs through the proton-proton chain of reactions given in Figure 1.4.

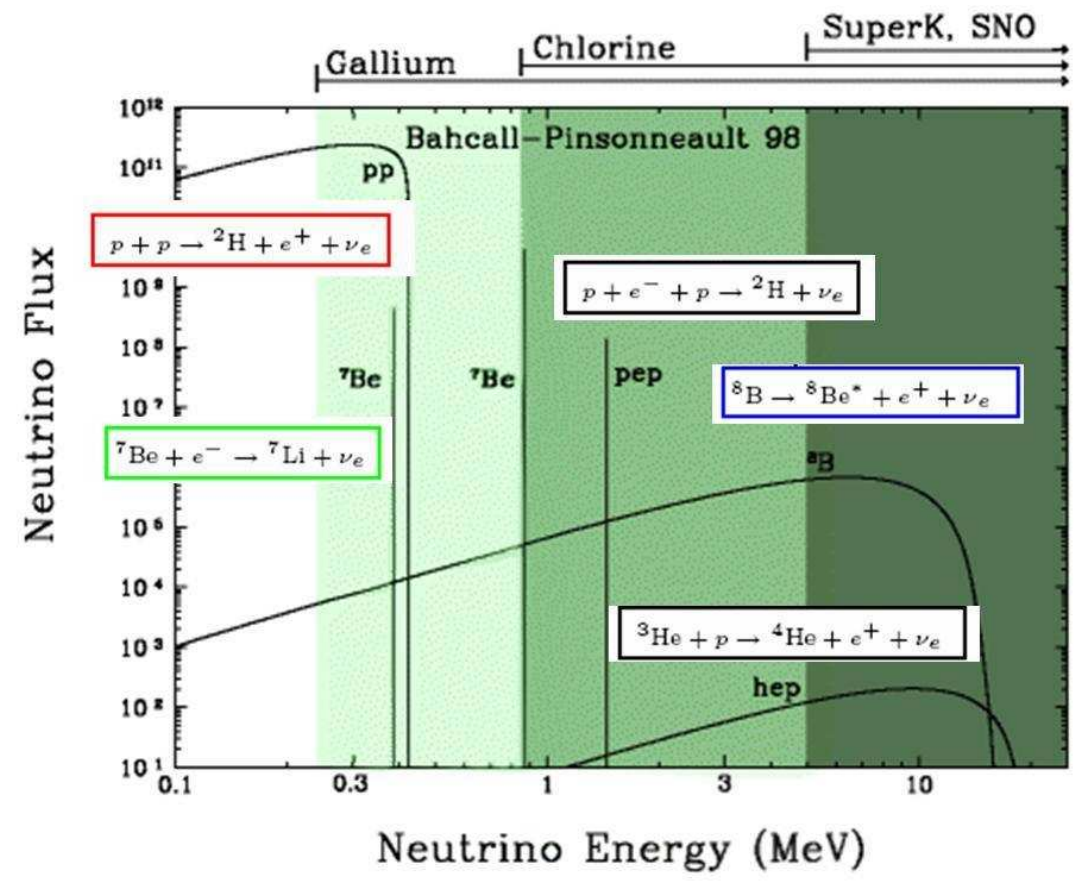

Figure 1.4: The Proton-Proton Chain of processes in the Sun

A unique test for this model was to measure the flux of neutrinos coming from the sun. Since Bahcall's model has an estimated uncertainty on the order of a few percent, the flux of electron neutrinos is an obvious test. Ray Davis' solar neutrino experiment in Homestake mine was the first experiment to test the validity of the Standard Solar Model [16] by measuring the flux of electron neutrinos from the sun. 
Davis' experiment was a 100000 gallon tank of industrial solvent based on chlorine, called perchlorethylene, $\mathrm{C}_{2} \mathrm{Cl}_{4}$. It was built 4800 feet underground in the Homestake Gold Mine in Lead, South Dakota, and ran continuously from 1970 to 1994. A large target deep underground was chosen due to the low probability of neutrino interaction and to shield the detector from other backgrounds. The experiment detected neutrinos through inverse $\beta$ decay:

$$
\nu_{e}+{ }^{37} \mathrm{Cl} \rightarrow e^{-}+{ }^{36} \mathrm{Ar}
$$

Once a month the tank was purged with helium to remove the argon atoms which were counted by detecting the decay back to chlorine with a half-life of 35 days. The experiment measured approximately one-third of the neutrinos that Bahcall's model predicted. Specifically the measured $\nu_{e}$ flux [16] was $2.56 \pm 0.16 \pm 0.16 \mathrm{SNU}$ compared to the predicted flux of $7.6_{-1.1}^{+1.3}$ SNU. One SNU (Solar Neutrino Unit) equals one neutrino interaction per second per $10^{36}$ atoms.

The scientific community could not reach a firm conclusion to the Solar Neutrino Deficit problem. Both Bahcall and Davis checked their work very intensely and claimed that no errors had been made. Numerous other experiments, such as Kamiokande in Japan [17], SAGE in the Soviet Union [18], GALLEX in Italy [19], and Super-Kamiokande [20] attempted to measure the electron neutrino flux from the sun and all came up with a deficit. It was finally SNO that solved the problem and was the first experiment to indirectly observe solar neutrinos changing flavor [22].

SNO was a water Cherenkov detector located 6800 feet underground at the Inco Limited's Creighton mine in Sudbury, Ontario, Canada. The detector was a tank consisting of 1 kton of ultra-pure heavy water $\left(D_{2} O\right)$ surrounded by over 9000 photomultiplier tubes that turned on in May 1999 and ended data taking in November 2006. Solar neutrinos were detected through three different processes: 


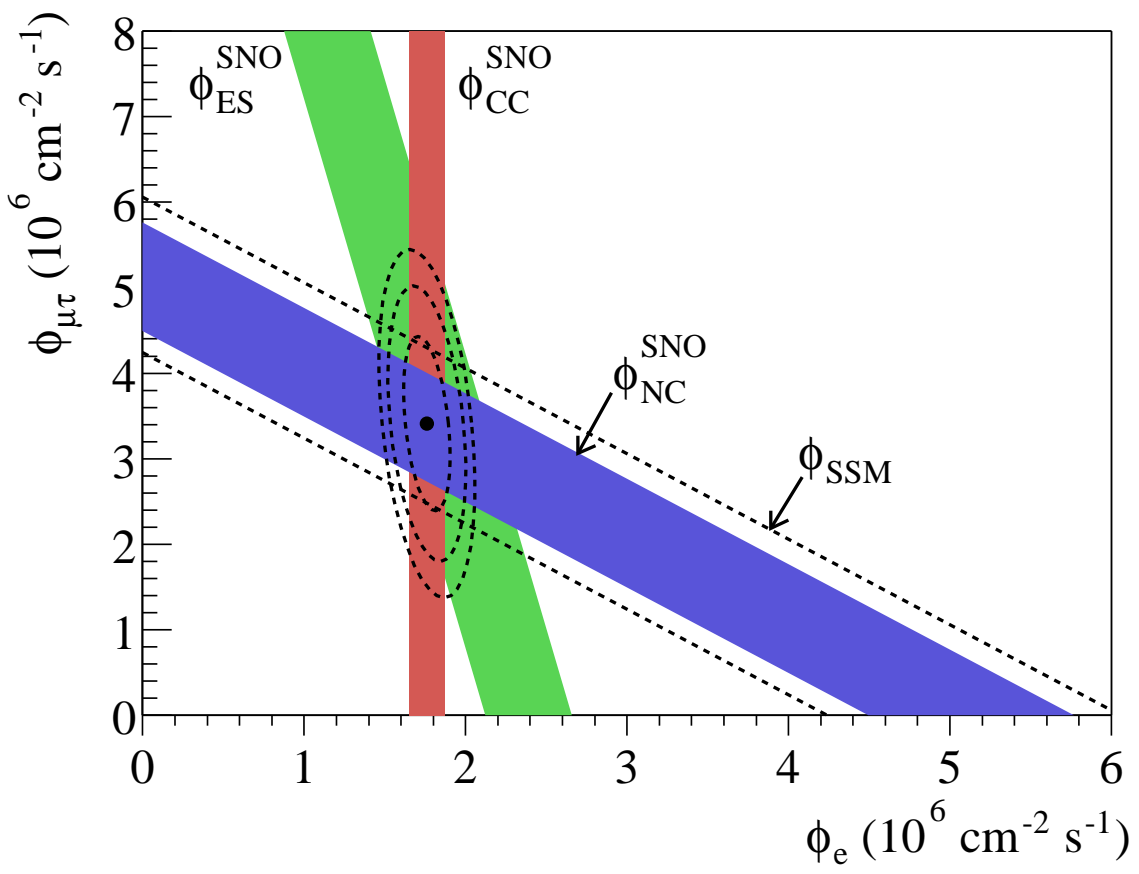

Figure 1.5: Flux ${ }^{8} \mathrm{~B}$ solar neutrinos which are $\mu$ or $\tau$ flavor vs flux of electron neutrinos deduced from the three neutrino reactions in SNO. the diagonal bands show the total ${ }^{8} \mathrm{~B}$ flux as predicted by the Standard Solar Model (dashed lines) and that measured with the NC reaction in SNO (solid band). The intercepts of these bands with the axes represent the $\pm 1 \sigma$ errors. The bands intersect at the fit values for the $\phi_{e}$ and $\phi_{\mu \tau}$, indicating that the combined flux results are consistent with neutrino flavor transformation assuming no distortion in the ${ }^{8} \mathrm{~B}$ neutrino energy spectrum [22].

$$
\begin{array}{ll}
\nu_{e}+\mathrm{d} \rightarrow \mathrm{p}+\mathrm{p}+\mathrm{e}^{-} & \text {Charged Current (CC), } \\
\nu_{x}+\mathrm{d} \rightarrow \mathrm{p}+\mathrm{n}+\nu_{x} & \text { Neutral Current (NC), } \\
\nu_{x}+\mathrm{e}^{-} \rightarrow \nu_{x}+e^{-} & \text {Elastic Scattering (ES). }
\end{array}
$$

The advantage that SNO possessed was the ability to simultaneously observe the disappearance of electron neutrinos and the appearance of other neutrino flavors through the neutral current interaction which is sensitive to all flavors. The mea- 
sured flux is shown in Figure 1.5. The combined results of SNO match that of the Standard Solar Model very well. By being able to measure neutrino flavors other than the electron type, SNO was able to add to the body of evidence that neutrinos do indeed oscillate.

\subsubsection{Reactor Experiments}

One of the by-products from a nuclear reactor is a high flux of neutrinos. For this reason, neutrino experiments can be built near reactors to take advantage of this free neutrino source. There were a number of early reactor experiments that found null results for oscillation scenarios. In 1979, F. Reines conducted another experiment again near Savannah River nuclear plant. He undertook the measurement of the ratio between neutral current and charged current interactions with anti-neutrinos on deuterium. The result was different from theoretical predictions and an explanation based on oscillations of neutrinos was considered [23], though the evidence for this explanation at the time was still inconclusive.

Several experiments then began a search for neutrino oscillations near nuclear plants. In 1984 two teams, Gosgen in Switzerland and Bugey in France undertook this search. Bugey saw oscillations [24], while Gosgen [26] did not. Bugey later retracted their result and finally gave a limit on oscillations [25].

The Chooz experiment was located about $1 \mathrm{~km}$ from the Chooz nuclear power plant and was designed to confirm solar observations. The detector was a 5 ton Gadolinium doped liquid scintillator detector. It detected $\bar{\nu}_{e}$ through inverse $\beta$ decay. The flux was compared with Monte Carlo and no evidence for oscillations were found, but Chooz's important contribution was to show that $\theta_{13}$ is very small [27]. Their result is shown in Figure 1.6 and the axes on the plot are $\Delta m_{23}^{2}$ and $\sin ^{2} 2 \theta_{13}$. Applying the results of Super-Kamiokande to Chooz's result of $\sin ^{2} 2 \theta_{13} \geq$ 0.17 at $90 \%$ CL gives a best limit of $\sin \theta_{13} \leq 0.035$ [28]. 


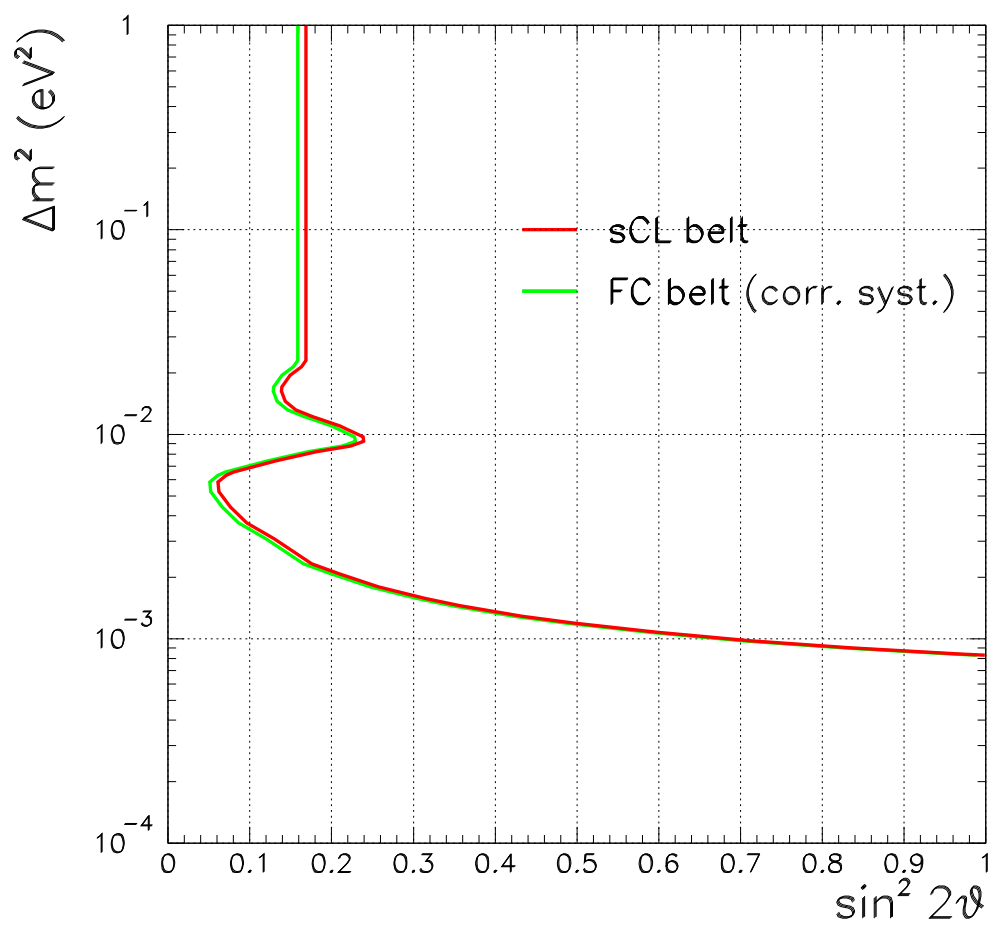

Figure 1.6: Exclusion plot at 90\% CL for the oscillation parameters from the Chooz experiment. The FC contour is obtained with taking systematic errors into account. $\vartheta$ is equal to $\theta_{13}$. [27]

KamLAND, or Kamioka Liquid scintillator Anti-Neutrino Detector, is another reactor experiment designed to confirm solar neutrino oscillation experiments. The experiment detected $\overline{\nu_{e}}$ from 53 different Japanese nuclear power stations at distances that varied between 150 and $210 \mathrm{~km} . \overline{\nu_{e}}$ are detected through charged current interactions. KamLAND's latest result [29] shows a clear distortion in their energy spectrum indicating a disappearance of $\overline{\nu_{e}}$. This spectrum and the corresponding contour are shown in Figure 1.7. Their final results give $\Delta m_{12}^{2}=$ $7.9_{-0.5}^{+0.6} \times 10^{-5} \mathrm{eV}^{2}$ and $\tan ^{2} \theta_{12}=0.40_{-0.07}^{+0.10}$. 


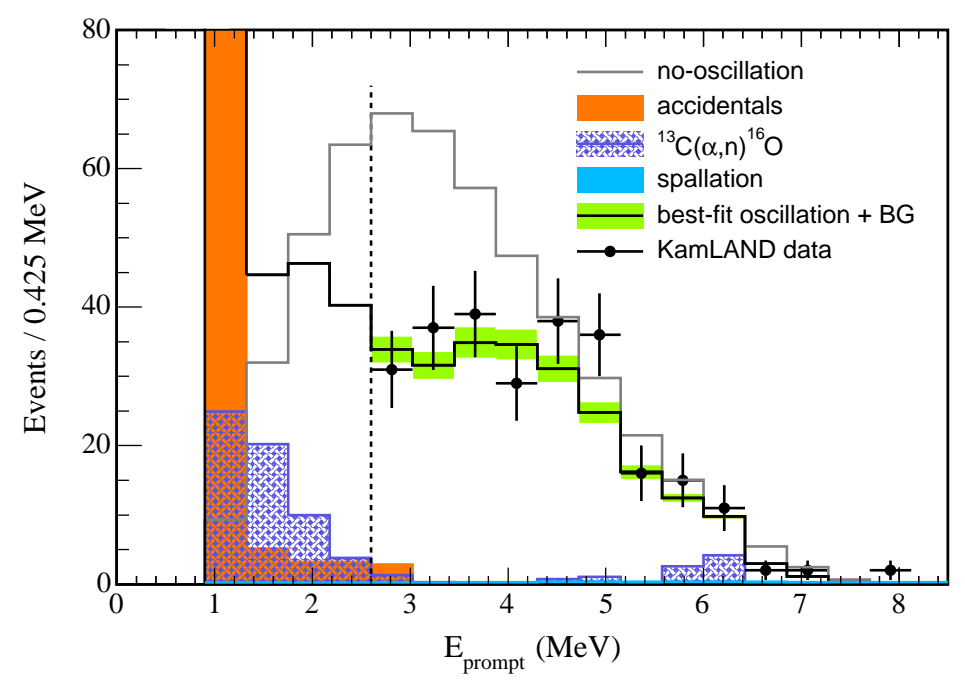

Figure 1.7: Neutrino oscillation results from KamLAND. The grey line in the top plot is the predicted energy spectrum in the absence of oscillations. The black dots are data with error bars and the solid black line is the best fit with a neutrino oscillation hypothesis. The deficit of neutrino events is clearly visible. The other colored components of the spectrum are backgrounds [29].

\subsubsection{Long-baseline Accelerator Experiments}

Long baseline experiments are designed to measure oscillation parameters to as high precision as possible. One usually first measures the neutrinos before oscillation, or near their production point and then at a sufficiently far distance allowing the neutrinos to oscillate. One of the advantages of very long baseline experiments is that the multiple node pattern may be detectable over the entire allowed range of $\Delta m^{2}$.

$\mathrm{K} 2 \mathrm{~K}$ in Japan was the first of these types of experiments with a baseline of $250 \mathrm{~km}$. The beam was produced at KEK and was directed towards the SuperKamiokande detector. There was a detector near the origin point that was a 1 kton Cherenkov detector. By comparing the energy spectra at the near detector and predicting what is expected in the Super-Kamiokande detector, one can measure 

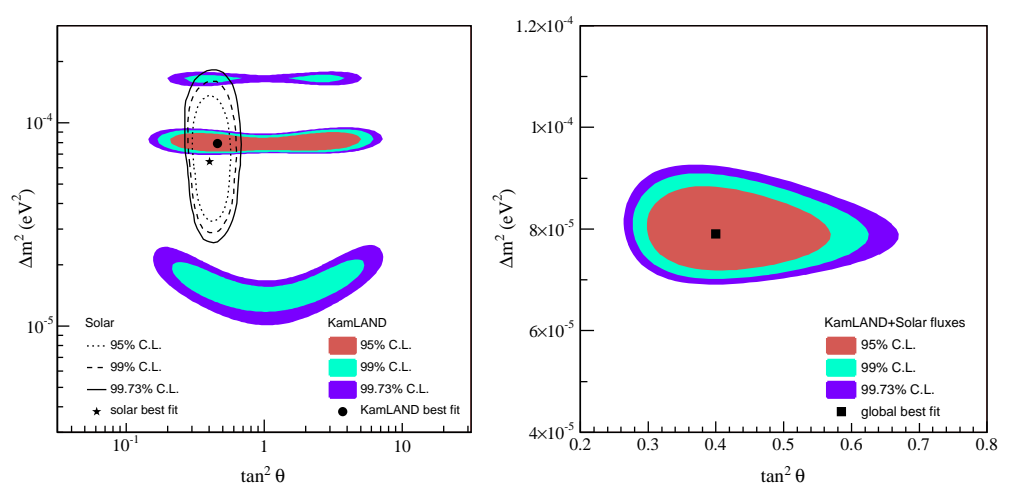

Figure 1.8: The left plot is the neutrino oscillation parameter allowed region from KamLAND anti-neutrino data (shaded regions) and solar neutrino experiments (lines). The right plot is the result of a combined two-neutrino oscillation analysis of KamLAND and the observed solar neutrino fluxes under the assumption of CPT invariance. the fit gives $\Delta m^{2}=7.9_{-0.5}^{+0.6} \times 10^{-5} \mathrm{eV}^{2}$ and $\tan ^{2} \theta=0.40_{-0.07}^{+0.10}$ including the allowed 1 -sigma parameter range [29].

the oscillation parameters. Super-Kamiokande observed 107 events compared to a Monte Carlo prediction of $151_{-10}^{+12}$ [30]. The energy spectrum and corresponding contour reported by K2K are shown in Figure 1.9.

MINOS is the second long baseline experiment based at Fermilab National Accelerator Laboratory [84]. The Main Injector at Fermilab uses $120 \mathrm{GeV}$ protons to produce a high intensity muon neutrino beam. The Near detector at Fermilab measures the $\nu_{\mu}$ energy spectrum before oscillations to make a prediction for the Far detector, located $735 \mathrm{~km}$ away in Soudan, MN. Details about the MINOS detectors will be elaborated in the third chapter of this work.

MINOS was designed to look at the same region in parameter space as Super-Kamiokande, the atmospheric neutrino sector. MINOS recently updated their first results that clearly show $\nu_{\mu} \rightarrow \nu_{\tau}$ oscillations and have made the most precise measurement of $\Delta m_{32}^{2}$ to date. The latest MINOS results give $\left|\Delta m_{32}^{2}\right|=$ $\left(2.38_{-0.16}^{+0.20}\right) \times 10^{-3} \mathrm{eV}^{2} / \mathrm{c}^{4}(68 \% \mathrm{CL})$ and $\sin ^{2} 2 \theta_{23}>0.84(90 \% \mathrm{CL})$ [31]. The Far 

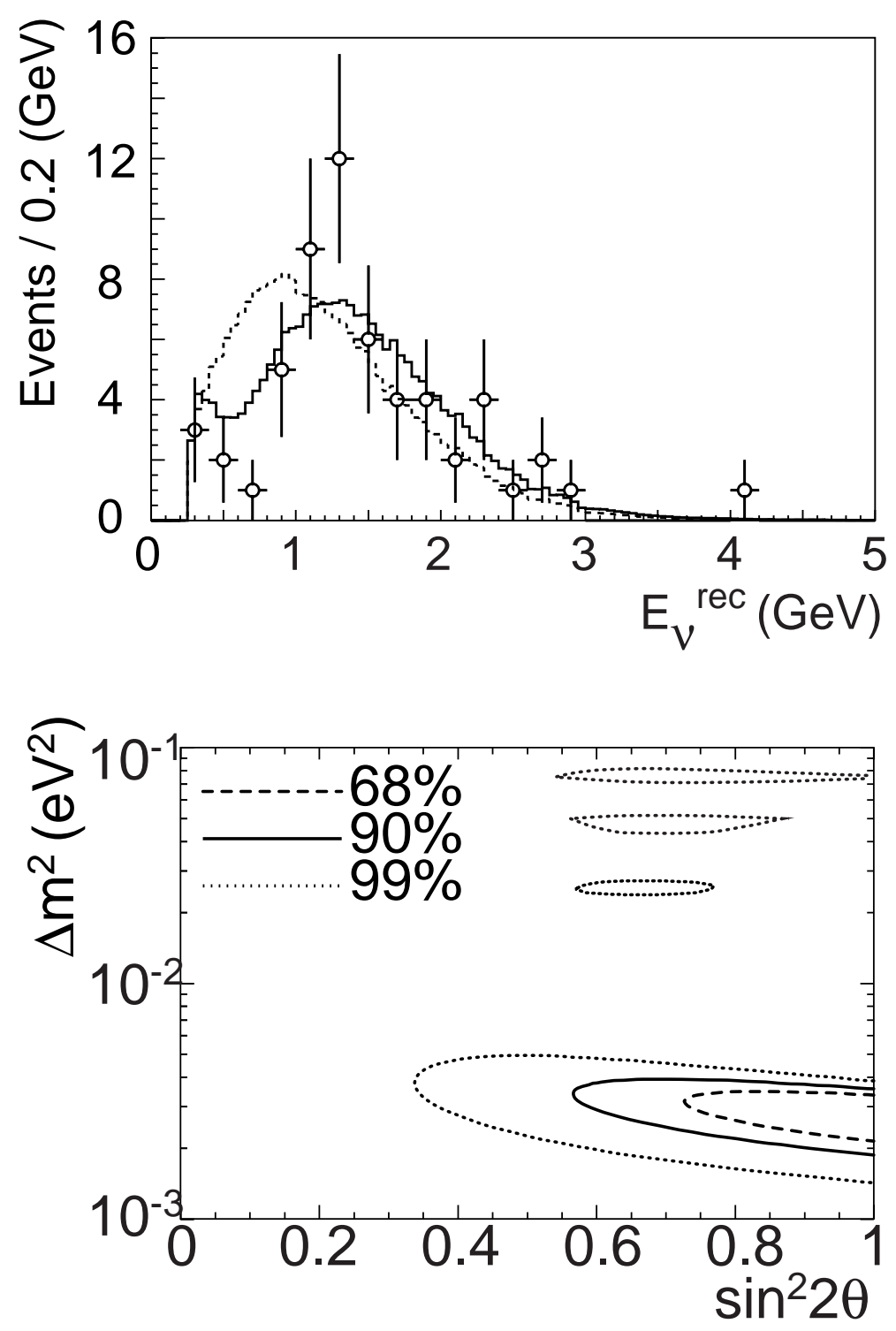

Figure 1.9: The top plot is the reconstructed $E_{\nu}$ distribution for the SK 1-ring $\mu$ like sample. Points with error bars are data. The solid line is the best fit spectrum. The dashed line is the expected spectrum without oscillation. These histograms are normalized by the number of events observed (57). The bottom plot is the resulting contour of allowed parameter space [30]. 

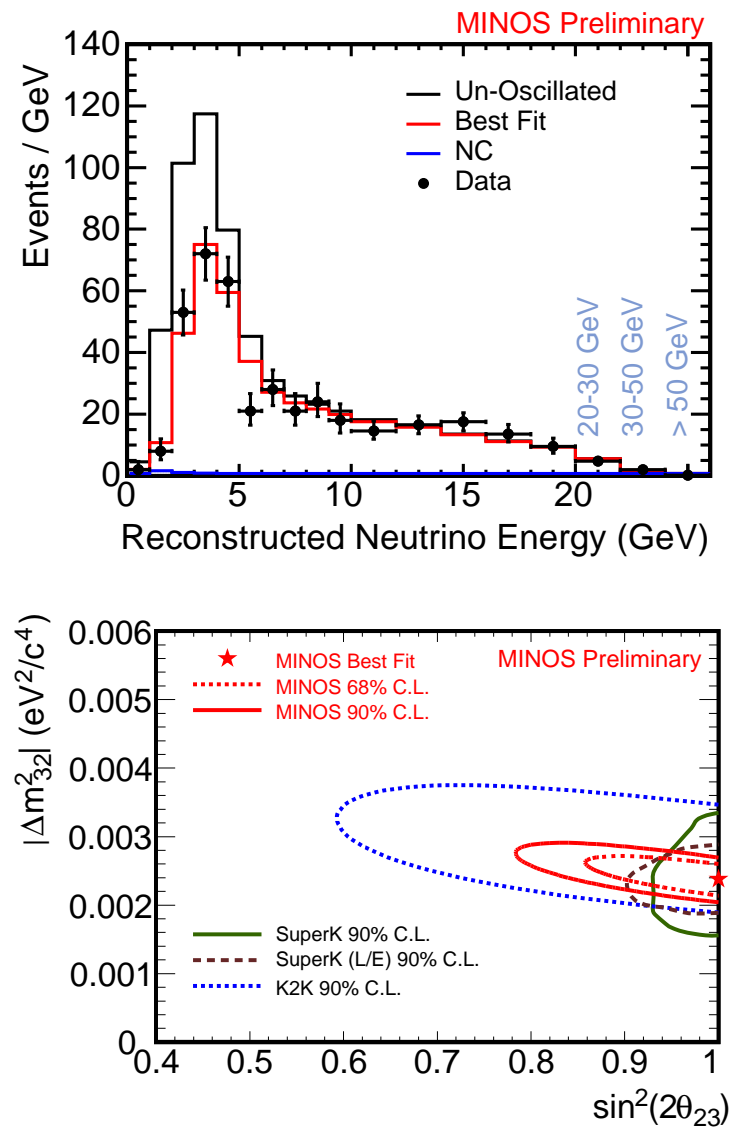

Figure 1.10: Recent MINOS oscillation results. The top figure shows the Far detector energy spectra. The black points are the data, the black line is the unoscillated Monte Carlo, and the red is the oscillation hypothesis best fit from the primary analysis. The small blue component is the neutral current background to the charged current spectra. The bottom figure is the parameter space showing Super-Kamiokande and K2K's results with the MINOS 68 and 90\% C.L. contour superimposed. [31] 
detector energy spectrum and contour are shown in Figure 1.10.

\subsubsection{Summary}

Neutrino oscillations are a very exciting and active field of research today in particle physics. The phenomenon is firmly established through the experimental results that have been discussed. It is without question that neutrinos oscillate, though there are still many outstanding questions in neutrino oscillation physics. How small is $\theta_{13}$ ? What is the sign of $\Delta m_{a t m}^{2}$ ? Are there neutrino to anti-neutrino oscillations occurring? Are neutrinos Majorana or Dirac particles?

Of particular interest to not only particle physics, but astrophysics and cosmology, is the possibility of the existence of sterile neutrinos. This would imply at least a fourth neutrino that contributes to neutrino oscillations. It is the sterile neutrino that we search for in this work and elaborate on in Chapter 2. 


\section{Chapter 2}

\section{Sterile Neutrinos}

\subsection{Introduction}

Briefly outlined in this chapter is the theoretical basis of incorporating sterile neutrinos into the Standard Model and how a sterile neutrino could have a wide range of masses. Despite the sterile neutrino's status as a hypothetical unobserved particle, there is a rich collection of theoretical works that would explain many physical phenomena should they be shown to exist. This is especially true in astrophysics and cosmology. Experimental limits on sterile neutrinos and ways to search for them are discussed. We end by elaborating on MINOS' sensitivity to sterile neutrinos and how we will search for them.

\subsection{Theoretical Considerations of Sterile Neutrinos}

The name sterile neutrino was suggested by Bruno Pontecorvo who hypothesized the existence of a right-handed neutrino [34]. It is a hypothetical neutrino that does not interact via any of the fundamental interactions of the Standard Model. Many models introduce sterile neutrinos to generate the three neutrino masses utilizing the see-saw mechanism [35]. This mechanism has a lagrangian described by 


$$
\mathscr{L}=\mathscr{L}_{S M}+\bar{\nu}_{a}\left(i \gamma^{\mu} \partial_{\mu}\right) \nu_{a}-y_{\alpha a} H \bar{L}_{\alpha} \nu_{\alpha}-\frac{M_{a}}{2} \bar{\nu}_{a}^{c} \nu_{a}+\text { h.c. }
$$

where $\mathscr{L}_{S M}$ is the Standard Model lagrangian which includes $n$ neutrino singlets $\nu_{a}(a=1, \ldots, n)$ with Yukawa couplings $y_{\alpha a} . \mathrm{H}$ is the Higgs doublet and $L_{\alpha}(\alpha=$ $e, \mu, \tau)$ are the lepton doublets. Current interest in sterile neutrinos stems from the fact that $n=2$ singlets are sufficient to explain the neutrino masses from atmospheric and solar neutrino experiments, but more are needed to explain LSND [36], r-process nucleosynthesis [37], pulsar kicks [38, 39], dark matter [41, 42, 43, 44] and the formation of supermassive black holes [45]. A sterile neutrino hypothesis can explain these phenomena.

Of physical interest is the scale of the right-handed Majorana masses $M_{a}$, which correspond to a sterile neutrino hypothesis, since they are currently unknown. The scale is possibly greater than the electroweak scale [35], or may be as low as a few eV [36, 44], but the possibilities span many orders of magnitude. The see-saw mechanism explains the small active neutrino masses in the presence of Yukawa couplings of order one, but requires $M_{a}$ to be larger than the electroweak scale. The outcome is that the masses of the three known neutrinos are suppressed by $\langle H\rangle / M_{a}$ where $\langle H\rangle$ is the expectation value from a spontaneously broken Higgs field.

Questions on the mass scale of sterile neutrinos can also be asked by looking at cosmology, a field where sterile neutrinos can provide interesting results. With a high-scale mass see-saw model, the baryon asymmetry can be generated from the out of equilibrium decays of heavy neutrinos [50]. For the low-scale mass see-saw model, the matter-antimatter asymmetry can be produced by neutrino oscillations [51]. While many see-saw models assume that sterile neutrinos have very large masses, making them unobservable, it is worthwhile to consider light $\left(M_{a}<100 \mathrm{eV}\right)$ sterile neutrinos not only because of the above arguments, but because they can explain certain experimental results. 


\subsection{Experimental Limits on the Existence of Sterile Neu- trinos}

Ultimately, truth ascribed to the number of theories that could support sterile neutrinos has to be verified by experiments. As elaborated above, there exist a wide range of mass possibilities for sterile neutrinos. Depending on what the mass actually is, they can be searched for in a number of different experiments. We discuss experimental results on the lighter mass scales first because these measurements are more active and current.

Lighter sterile neutrinos, with masses below $100 \mathrm{eV}$, can be discovered by one of many neutrino oscillation experiments [54]. Currently the LSND (Liquid Scintillator Neutrino Detector) collaboration has reported a signal that would imply at least a fourth species of neutrino [55], which in combination with other experiments may imply the possibility of two sterile neutrinos [56]. This result also supports the possibility that sterile neutrino decays, rather than neutrino oscillations, explain the LSND result [57]. It should be noted that the KARMEN experiment used $\bar{\nu}_{\mu}$ from $\mu^{+}$decay at rest to search for $\bar{\nu}_{\mu} \rightarrow \bar{\nu}_{e}$ oscillations as did LSND, and measured 15 candidates that passed the conditions for the $\bar{\nu}_{e}$ signature, in agreement with their background expectation of $15.8 \pm 0.5$ events, yielding no indication for oscillations [60].

LSND was a liquid scintillator detector located at the Los Alamos Neutron Science Center that conducted a search for $\bar{\nu}_{\mu} \rightarrow \bar{\nu}_{e}$ oscillations using $\bar{\nu}_{\mu}$ from $\mu^{+}$ decay at rest. The collaboration observed a total excess of $87.9 \pm 22.4 \pm 6.0$ events consistent with $\bar{\nu}_{e} p \rightarrow e^{+} n$ scattering above their expected background. This excess corresponds to an oscillation probability of $(0.264 \pm 0.067 \pm 0.045) \%$. This data suggest that neutrino oscillations occur in the $0.2-19 \mathrm{eV}^{2} / c^{4} \Delta m^{2}$ range, indicating a neutrino mass greater than $0.4 \mathrm{eV} / c^{2}$. The spectrum of excess $\bar{\nu}_{e}$ events used to extract a signal reported by LSND and the $90 \%$ CL contour for $\left(\sin ^{2} 2 \theta, \Delta m^{2}\right)$ is 
shown in Figure 2.1.

The interest in the LSND result suggested an experiment to verify LSND be constructed. It was also suggested that this experiment should have the same sensitivity as LSND, but done independently. This experiment is called MiniBoonNE (Booster Neutrino Experiment) [58]. A $\nu_{\mu}$ neutrino beam at the Fermilab Booster is produced from $8 \mathrm{GeV}$ protons incident on a $71 \mathrm{~cm}$ long by $1 \mathrm{~cm}$ diameter beryllium target. The beam is directed at a detector filled with 800 tons of mineral oil and lined with 1280 photomultiplier tubes. It should be noted that though MiniBooNE is very similar in design and even looks at the same $L / E_{\nu}$ region as LSND, it initially used neutrinos instead of anti-neutrinos for its first results in March 2007. Since January 2007 MiniBooNE has switched their beam to anti-neutrinos.

The $\nu_{\mu}$ energy spectrum measured by MiniBooNE peaks at $700 \mathrm{MeV}$ and extends to approximately $3000 \mathrm{MeV}$. Integrating over the neutrino flux, interactions in MiniBooNE are mostly charged current quasi-elastic (CCQE) scattering (39\%), neutral current (NC) elastic scattering (16\%), charged current (CC) single pion production (29\%), and NC single pion production (12\%). Multi-pion and deepinelastic scattering contributions are $<5 \%$.

MiniBooNE presented their first results in late March 2007 and reported no evidence for $\nu_{\mu} \rightarrow \nu_{e}$ oscillations in the LSND region, refuting a simple two neutrino oscillation interpretation of the LSND results [59]. The predicted and measured energy spectrum is shown in Figure 2.2 and the allowed regions are in Figure 2.3. MiniBooNE observed 380 events between $475<E_{\nu}^{Q E}<1250$ with a prediction of $358 \pm 19$ (stat) \pm 35 (sys) events. This corresponds to a $0.55 \sigma$ excess over a no oscillations background. In parameter space the measured values were $\sin ^{2}(2 \theta)=1.1 \times 10^{-3}$ and $\Delta m^{2}=4.1 \mathrm{eV}^{2} / c^{4}$. 

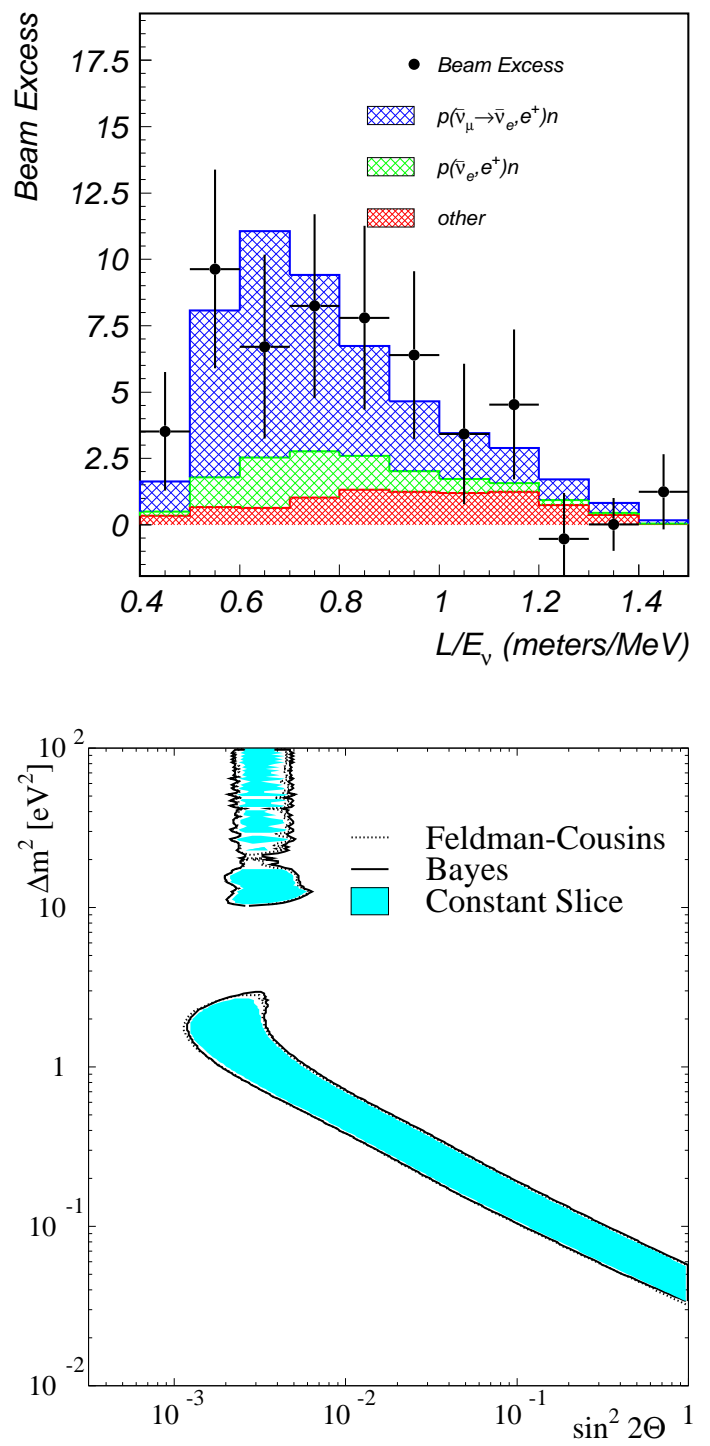

Figure 2.1: The top plot is the $L / E_{\nu}$ distribution for electron neutrino events in the LSND detector. The beam excess implies oscillation from $\nu_{\mu} \rightarrow \nu_{e}$. The bottom plots is the LSND favored regions in the $\left(\sin ^{2} 2 \theta, \Delta m^{2}\right)$ plane at $90 \% \mathrm{CL}$. The Feldman-Cousins, Bayesian, and constant-slice methods all give about the same result [55]. 
MiniBooNE performed a blind analysis in which the presumed signal region of their data was hidden until the rest of the data was understood. Once this was condition was satisfied and the analysis cuts were set, the oscillation analysis was performed on the full data set. Originally, the full two neutrino oscillation fit was done in the range $300<E_{\nu}^{Q E}<3000 \mathrm{MeV}$ and, with no information on the fit parameters revealed, the sum of the predicted background and simulated best fit signal was compared to data in several variables, returning only the $\chi^{2}$. While agreement was good in most of the comparisons, the energy spectrum had a $\chi^{2}$ probability of only $1 \%$. Thus the MiniBooNE collaboration further investigated their backgrounds, focusing on the lowest energies where $\nu_{\mu}$ induced backgrounds are large. Part of the study included the unsigned bin-by-bin fractional discrepancies in the energy spectrum. This gave the collaboration suspicion about the low energy region since it specifically has a large deviation, as seen in Figure 2.2. They finally concluded that while there were no specific problems with the background estimates, it was found that raising the minimum $E_{\nu}^{Q E}$ of the fit region to $475 \mathrm{MeV}$ greatly reduced a number of backgrounds with little impact on the fit's sensitivity to oscillations. Thus the oscillation fits were performed in the energy range 475 $<E_{\nu}^{Q E}<3000 \mathrm{MeV}$. As seen in Figure 2.2, the cause of this low $\chi^{2}$ probability is a significant excess of data events below $475 \mathrm{GeV}$ [59]. Currently, more advanced analyses of their data are being undertaken by the collaboration to understand this excess. 


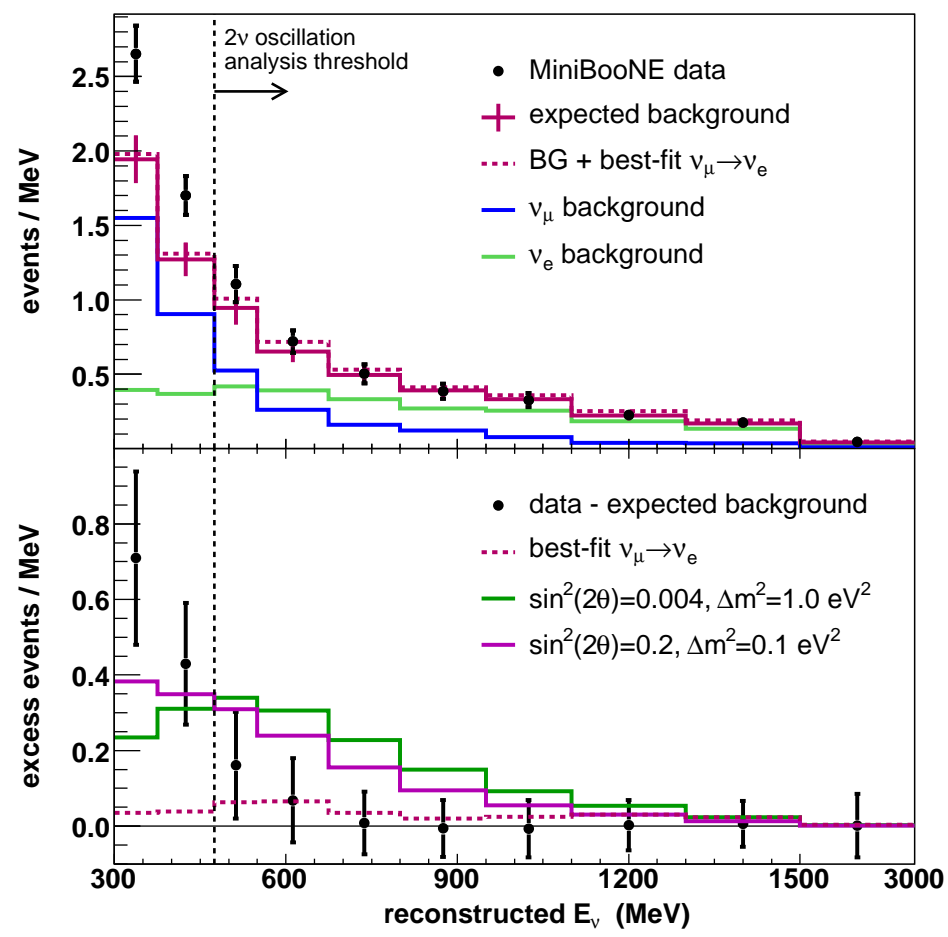

Figure 2.2: The MiniBooNE oscillation results. The top plot shows the number of candidate $\nu_{e}$ events as a function of $E_{\nu}^{Q E}$. The points represent the data with statistical error, while the histogram is the expected background with systematic errors from all sources. The vertical dashed line indicates the threshold used in the two-neutrino oscillation analysis. Also shown are the best-fit oscillation spectrum (dashed histogram) and the background contributions from $\nu_{\mu}$ and $\nu_{e}$ events. The bottom plot shows the number of events with the predicted background subtracted as a function of $E_{\nu}^{Q E}$, where the points represent the data with total errors and the two histograms correspond to LSND solutions at high and low $\Delta m^{2}[59]$ 


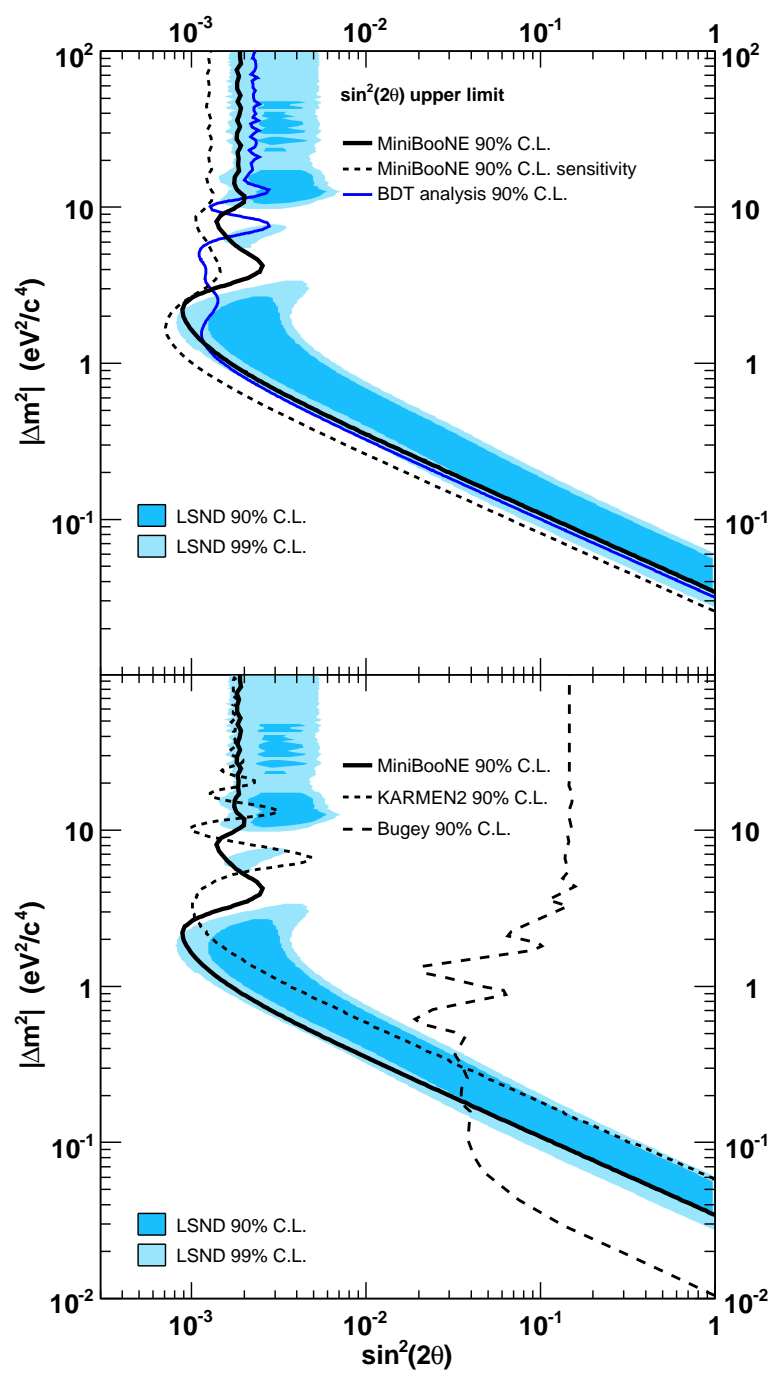

Figure 2.3: The MiniBooNE allowed oscillation parameter regions [59]. The top plot shows the $90 \%$ CL limit (thick solid curve) and sensitivity (dashed curve) for events with $475<E_{\nu}^{Q E}<3000 \mathrm{MeV}$ within a two neutrino oscillation model. Also shown is the limit from the boosted decision tree analysis (thin solid curve) for events with $300<E_{\nu}^{Q E}<3000 \mathrm{MeV}$. The bottom plot shows the limit from the KARMEN [60] and Bugey [61] experiments. The shaded areas show the $90 \%$ and $99 \%$ CL allowed regions from the LSND experiment. 
Another experimental result with respect to sterile neutrinos is given by the Super-Kamiokande Experiment. As was discussed in the first chapter, SuperKamiokande provided the first direct evidence that neutrinos do indeed oscillate [21]. Super-Kamiokande specifically interpreted their results at the time as due to $\nu_{\mu} \rightarrow \nu_{\tau}$ oscillations, although their data did not distinguish whether the muon neutrinos were oscillating into tau neutrinos or sterile neutrinos, as both hypotheses fit the data. They used three complementary data samples to study the differences in the zenith angle distribution due to neutral currents and matter effects. The collaboration has produced a result [62] that finds no evidence favoring only sterile neutrinos, and reject the hypothesis at the $99 \%$ confidence level. These results are quantified in Figure 2.4.

Even if the Super-Kamiokande data does not specifically favor oscillations into only $\nu_{s}$, one may ask the question whether some combination of both $\nu_{\tau}$ and $\nu_{s}$ is taking place. Super-Kamiokande analyzed their results in a two neutrino scheme because $\theta_{13}$ is small and the $\Delta m_{\text {atm }}^{2}$ and $\Delta m_{\text {sol }}^{2}$ are essentially decoupled from each other, which means only $\nu_{\mu} \longleftrightarrow \nu_{\tau}$ oscillations in the absence of a sterile neutrino. But how does one account for the fact that a sterile neutrino could exist? The solution is to keep a two neutrino scheme with $\nu_{\mu} \longleftrightarrow \nu_{x}$ where $\nu_{x}=\left(\cos \xi \nu_{\tau}+\sin \xi \nu_{s}\right)$ and $f_{\text {sterile }}=\sin ^{2} \xi$. The manner of looking at this prescription of $\tau$ and sterile admixture was defined in [64]. One can write the relative oscillation probabilities as

$$
\begin{aligned}
& P\left(\nu_{\mu} \rightarrow \nu_{\mu}\right)=1.0-\sin ^{2}(2 \theta) \sin ^{2}\left(1.27 L \Delta m_{\text {atm }}^{2} / E_{\nu}\right) \\
& P\left(\nu_{\mu} \rightarrow \nu_{\tau}\right)=\left(1.0-f_{\text {sterile }}\right) \sin ^{2}(2 \theta) \sin ^{2}\left(1.27 L \Delta m_{\text {atm }}^{2} / E_{\nu}\right) \\
& P\left(\nu_{\mu} \rightarrow \nu_{s}\right)=f_{\text {sterile }} \sin ^{2}(2 \theta) \sin ^{2}\left(1.27 L \Delta m_{\text {atm }}^{2} / E_{\nu}\right)
\end{aligned}
$$



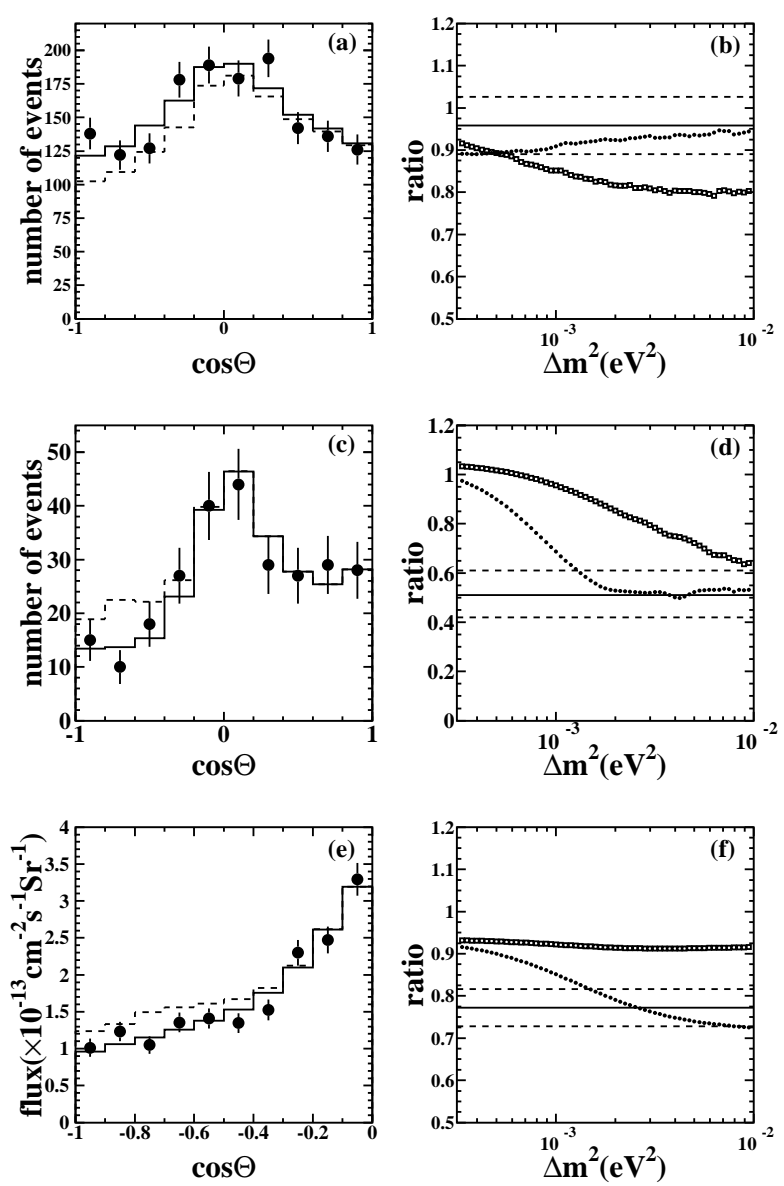

Figure 2.4: (a,c,e) Zenith angle distributions of atmospheric neutrino events satisfying cuts described in the text: (a) multi-ring sample, (c) partially contained sample, and (e) upward through-going muon sample. The black dots indicate the data and statistical errors. The solid line indicates the prediction for $\nu_{\mu} \leftrightarrow \nu_{\tau}$, and the dashed for $\nu_{\mu} \leftrightarrow \nu_{s}$, with $\left(\Delta m^{2}, \sin ^{2} 2 \theta\right)=\left(3.2 \times 10^{-3} \mathrm{eV}^{2}, 1\right)$. The two predictions are normalized by a common factor so that the number of the observed events and the predicted number of events for $\nu_{\mu} \leftrightarrow \nu_{\tau}$ are identical. (b,d,f) Expected value of the corresponding test ratio as a function of $\Delta m^{2}$. The solid horizontal lines indicates the measured value from the Super-Kamiokande data with statistical uncertainty indicated by dashed lines. Black dots indicate the prediction for $\nu_{\mu} \leftrightarrow \nu_{\tau}$, and empty squares for $\nu_{\mu} \leftrightarrow \nu_{s}$, in both cases for maximal mixing [62]. 


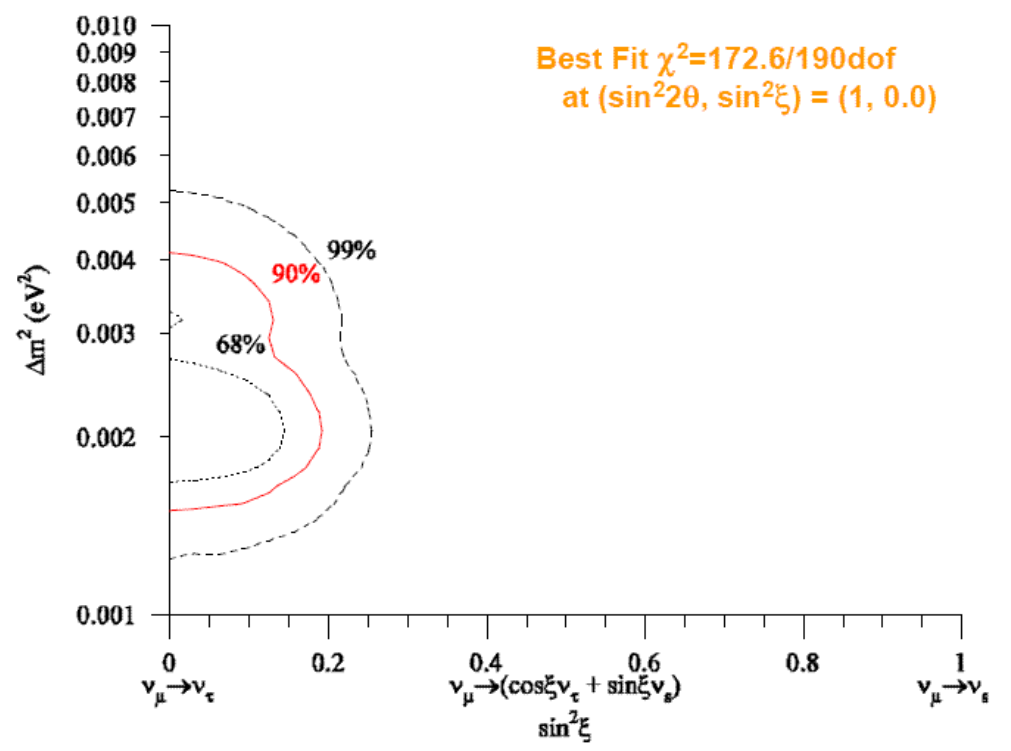

Figure 2.5: Latest Super-Kamiokande results on $f_{\text {sterile }}$ vs $\Delta m^{2}$ [63].

One then fits their data for three parameters, $\Delta m^{2}, \sin ^{2}(2 \theta)$, and $f_{\text {sterile. }}$. The physical meaning of $f_{\text {sterile }}$ is the fraction of $\nu_{\mu}$ neutrinos that oscillate into $\nu_{s}$ rather than $\nu_{\tau}$. A non-zero value would indicate the existence of a sterile neutrino. The latest value is given by the Super-Kamiokande collaboration in Figure 2.5 which has a best fit of $f_{\text {sterile }}=0[63]$.

Currently, all experimental searches for sterile neutrinos have come up with a null result. The only exception is the LSND result which is highly disfavored due to the latest MiniBooNE results. There is still a chance that MiniBooNE maybe able to explain their excess of events at low energies with some sterile neutrino prescription. These highly circulated results have been in the $<100 \mathrm{eV}$ mass range for sterile neutrinos, while searches for higher mass ranges may yet yield more results. 


\subsection{Sterile Neutrinos in Astrophysics and Cosmology}

Astrophysical and cosmological observations can also help with understanding sterile neutrinos. The hypothesis of a light $\left(M_{a}<100 \mathrm{eV}\right)$ sterile neutrino, consistent with the LSND result, agrees with existing bounds on the big-bang nucleosynthesis [52, 53] and large-scale structure. This is especially true if the mixing lepton asymmetry of the universe is larger than the baryon asymmetry [70].

More importantly, sterile neutrinos are good candidates for cosmological dark matter [41, 42, 43, 44]. According to the lagrangian in Equation 2.1, the production of relic sterile neutrinos via the Dodelson-Widrow mechanism [41] allows the correct amount to account for all dark matter if one of the Majorana masses is of the order of a keV. The mass and mixing angle of sterile neutrinos are subject to x-ray limits on the photons from the decays of relic sterile neutrinos [74] in addition to the Lyman- $\alpha$ bound [75].

Hypothetical relic sterile neutrinos from the Big Bang can decay into lighter ones and x-ray photons [76] which can be detected by x-ray telescopes. The flux of these x-rays depends on the sterile neutrino abundance. If all the dark matter is due to sterile neutrinos, $\Omega_{s} \approx 0.2$, then the limit on the mass and the mixing angle of sterile neutrinos is given by the dashed line in Figure 2.6. The interactions given in Equation 2.1 cannot produce such sterile neutrinos unless there is a large lepton asymmetry in the universe [44]. A model-independent bound [73] based on this scenario is shown as a solid (purple) region in Figure 2.6. It is based on the flux limit from [74] and the analytical fit to the numerical calculation of the sterile neutrino production by [77].

The x-ray photons from sterile neutrino decays in the early universe could have affected star formation. Though these x-rays are not sufficient to reionize the universe, they can catalyze the production of molecular hydrogen and speed up star formation [46], which could cause the reionization. Molecular hydrogen is a cooling 


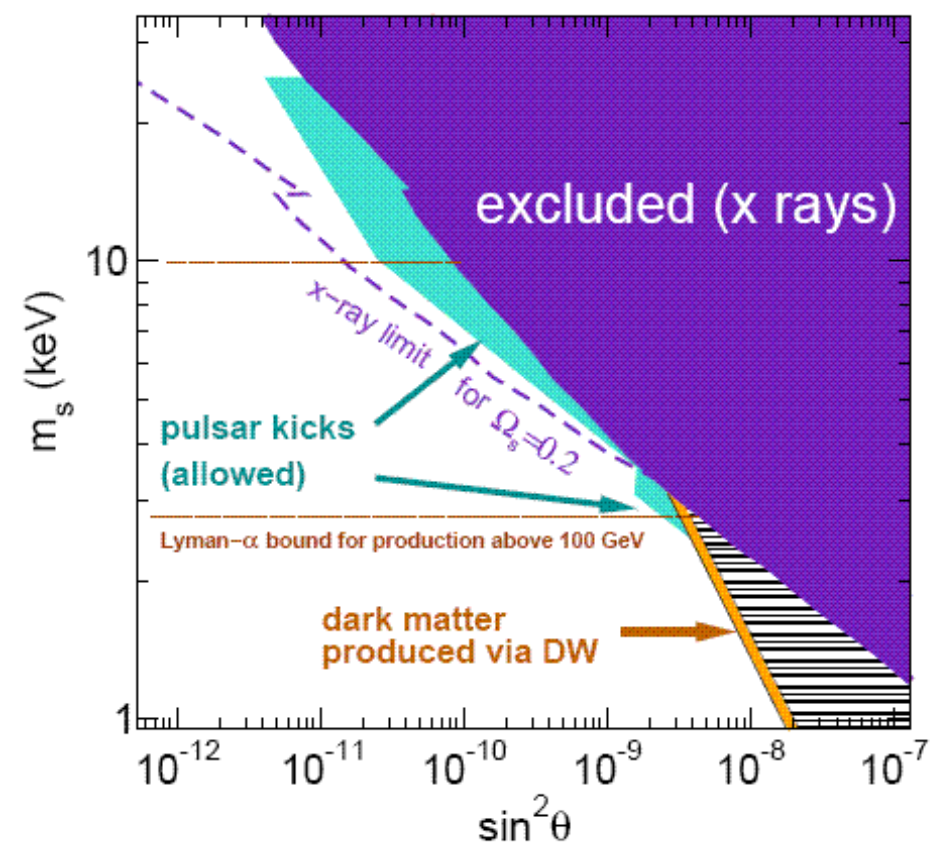

Figure 2.6: The allowed astrophysical and cosmological bounds for sterile neutrinos. The $\mathrm{y}$-axis is the mass of the sterile neutrino while $\sin \theta$ on the $\mathrm{x}$-axis is defined as $\frac{y\langle H\rangle}{m_{s}}$ where $\mathrm{y}$ is the Yukawa coupling, $\langle H\rangle$ is the expectation value of a spontaneously broken Higgs field. Sterile neutrinos with masses 2-25 keV can explain pulsar kicks if the mixing angles are large enough. In the region marked excluded (x-rays), the relic sterile neutrinos produced in neutrino oscillations via the Dodelson-Widrow (DW) mechanism would have a density inconsistent with the existing x-ray bounds. If the sterile neutrinos constitute all the dark matter, their masses and mixings should fall below the dashed line: a large lepton asymmetry [43] or a new production mechanism $[72,73]$ is required. The lyman- $\alpha$ bound for dark-matter sterile neutrinos produced at temperatures $\mathrm{T}>100 \mathrm{GeV}$ is $m_{s}>2.7 \mathrm{keV}$ [73]. The cosmological and the $\mathrm{x}$-ray bounds do not apply if the universe was never reheated above $\mathrm{T} \approx$ $\mathrm{MeV}$ [71]. Plot taken from [69]. 
agent necessary for the collapse of primordial gas clouds that gave birth to the first stars. The fraction of molecular hydrogen must exceed a certain minimal value for the star formation to begin. The ionization fraction determines the rate of molecular hydrogen production. If dark matter is made up of sterile neutrinos, their decays produce a sufficient flux of photons to increase the ionization fraction by as much as two orders of magnitude [46].

Decays of these relic sterile neutrinos could produce an observable signature in the 21-cm background [78] which can be studied by Low Frequency Array (LOFAR), the 21 Centimeter Array (21CMA), the Mileura Wide-field Array (MWA) and the Square Kilometer Array (SKA).

Observations of neutrinos from SN1987A set limits on the amount of energy that sterile neutrinos can take out of the supernova, but they are still consistent with them carrying away as much as half of the total energy of the supernova. More analysis shows that the emission of sterile neutrinos from a cooling newly born neutron star would be anisotropic due to the star's magnetic field [79, 80]. The anisotropy of this emission can result in recoil velocity of the neutron star as high as $10^{3} \mathrm{~km} / \mathrm{s}$, hence they give the recoiling neutron star a momentum large enough to explain pulsar kicks for the neutrino emission anisotropy as small as a few percent $[79,80]$. This mechanism can be the explanation of observed pulsar velocities [81]. The range of masses and mixing angles required to explain the pulsar kicks is shown in 2.6.

Though the existence of a sterile neutrino is still speculative, it is clear that the astrophysical and cosmological interest in sterile neutrinos is great and hints of their existence would help solve many outstanding problems in these fields. 


\subsection{Searching for Sterile Neutrinos in MINOS}

MINOS has the capability to set a limit on $f_{\text {sterile }}$, the fraction of sterile neutrinos that contribute to atmospheric oscillations. As described in the previous section, we follow the definition used by Super-Kamiokande in that we define $\nu_{\mu} \longleftrightarrow \nu_{x}$ where $\nu_{x}=\left(\cos \xi \nu_{\tau}+\sin \xi \nu_{s}\right)$ and $f_{\text {sterile }}=\sin ^{2} \xi$ defined by [64].

MINOS makes it's measurement of $\Delta m^{2}$ and $\sin ^{2}(2 \theta)$ by observing a disappearance in charged current $\nu_{\mu}$ 's interactions at the Far detector. This is justified because $\nu_{\mu}$ 's that oscillate into sterile neutrinos will not interact by definition, and if they oscillate into $\nu_{\tau}$ 's, interactions will be heavily suppressed due to the $\tau$ production threshold and phase space suppression associated with the heavy $\tau$. Charged current events alone do not constrain $f_{\text {sterile }}$.

Neutral current events are used in MINOS to measure $f_{\text {sterile. The neutral }}$ current cross section is the same for $\nu_{\mu}$ and $\nu_{\tau}$, hence if $\nu_{\mu}$ 's only oscillate into $\nu_{\tau}$ 's, then one expects no deficit in the neutral current energy spectrum at the Far detector. If there exists a sterile component to atmospheric oscillations, one will be able to measure it by looking for a deficit in the number of neutral current interactions compared with the predicted number.

We perform a measurement of neutral current visible energies in order to deduce $f_{\text {sterile }}$ in MINOS. A outlook on the projected sensitivity of MINOS to $f_{\text {sterile }}$ is shown in Figure 2.7. The contours in Figure 2.7 were generated by simulating $2.5,6.5$, and $13.0 \times 10^{20}$ protons on target worth of data in the MINOS Far Detector with input values of $f_{\text {sterile }}=0, \Delta m^{2}=0.0027 \mathrm{eV}^{2} / \mathrm{c}^{4}$, and $\sin ^{2}(2 \theta)=1.0$. The top plot is the resulting 90\% CL and the bottom plot is $99 \% \mathrm{CL}$. The more protons on target MINOS receives, the smaller the error on $f_{\text {sterile. }}$ One can also compare the plot to Super-Kamiokane's measurement of $f_{\text {sterile }}$ shown in Figure 2.5. MINOS can be competitive with Super-Kamiokande's result when the total protons on target reaches at least $13.0 \times 10^{20}$. The measurement is an important analysis 
because MINOS uses a neutrino beam produced at an accelerator (Fermilab) and thus would be an independent measurement of $f_{\text {sterile }}$ in the atmospheric sector. In the next chapter we discuss the MINOS detectors and the technology used to measure neutrino interactions.
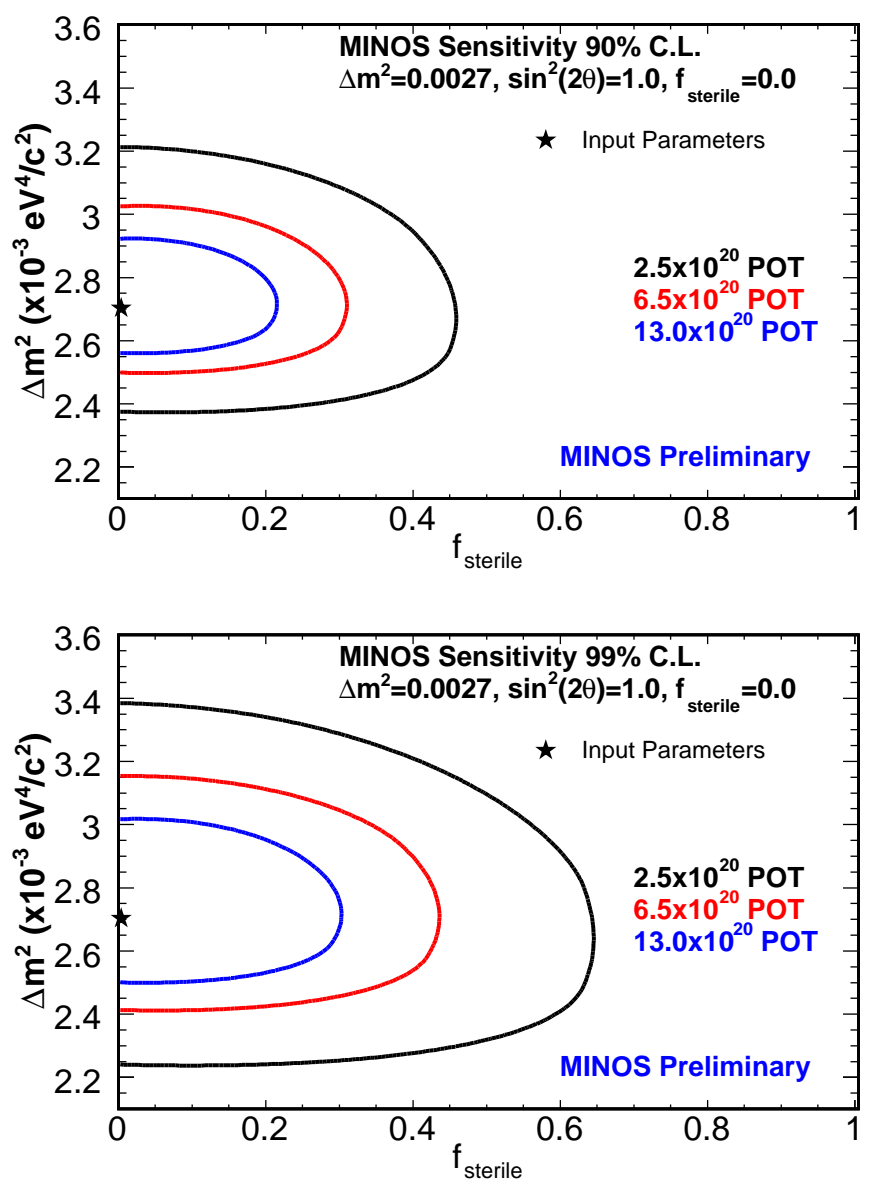

Figure 2.7: The top plot is the 90\% C.L. projected MINOS sensitivity for three different values of POT. The bottom plot is the projected $99 \%$ C.L. for three different values of POT. The input values are $\Delta m^{2}=0.0027 \mathrm{eV}^{2} / \mathrm{c}^{4}, \sin ^{2}(2 \theta)=1.0$ and $f_{\text {sterile }}=0.0$, which is given by the star in each plot. 


\section{Chapter 3}

\section{The MINOS Experiment}

\subsection{Overview}

The MINOS experiment uses a neutrino beam produced at Fermilab National Accelerator Laboratory (Fermilab) and two steel-scintillator sampling calorimeter detectors designed to measure neutrino interactions. The main purpose of MINOS is to accurately measure the oscillation parameters $\Delta m_{a t m}^{2}$ and $\sin ^{2}\left(2 \theta_{a t m}\right)$ in the region reported by Super-Kamiokande. The first detector, the Near detector, is located $1040 \mathrm{~m}$ from the creation of the neutrino beam and will measure the energy spectrum of $\nu_{\mu}$ 's before they have a chance to oscillate. Neutrinos travel 735 $\mathrm{km}$ to the Soudan Mine in Soudan, Minnesota, where the Far detector is located, and the oscillated energy spectrum is measured. See Figure 3.1 for a map of the neutrino's path from Fermilab to Soudan. By using the Near detector to predict what one expects in the Far detector, MINOS is able to accurately measure the atmospheric oscillation parameters using charged current interactions and the fraction of neutrinos that oscillation into sterile neutrinos, $f_{\text {sterile }}$, using neutral current interactions. The neutrino beam line is described in detail in the NuMI technical design report [83]. The MINOS experiment has a separate technical design report 


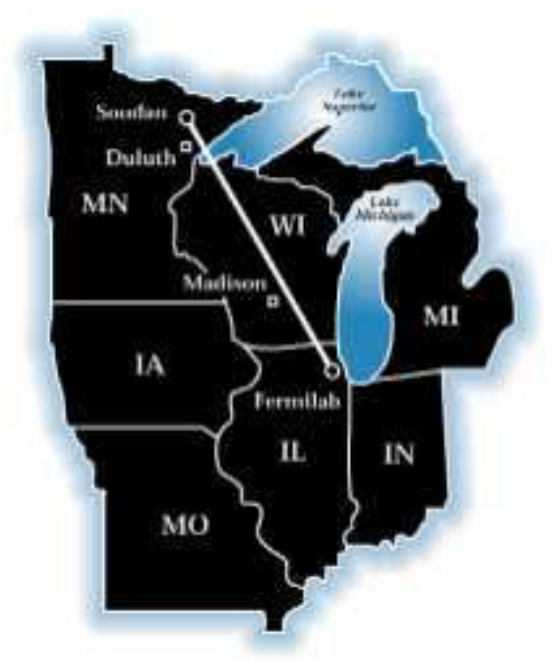

Figure 3.1: A geographical picture of the MINOS experiment. Neutrinos are produced at Fermilab and go through the Near detector, measuring the unoscillated energy spectrum, then the neutrinos travel $735 \mathrm{~km}$ to Soudan mine to be detected, in their oscillated form, at the Far detector.

that documents the details of the MINOS detectors and construction [84].

We begin by discussing the creation of the neutrino beam. Next we talk about calorimetry and detector technology since it is similar between the Near and Far detectors. Last we have two sections describing the differences between the Near and Far detectors, and brief outline on the Calibration detector.

\subsection{Neutrino Beam}

The "Neutrinos at the Main Injector" (NuMI) beam line was constructed at Fermi National Accelerator Laboratory to deliver a powerful muon neutrino beam to the MINOS experiment. Protons with an energy of $120 \mathrm{GeV}$ from the Main Injector are extracted with a designed spill duration of $8.6 \mu$ sec toward a target hall. The beamline is designed for $4 \times 10^{13}$ protons per pulse (ppp). The protons are then focused onto a graphite target that is $6.4 \times 15.0 \times 940.0 \mathrm{~mm}^{3}$ in size. The designed 
Table 3.1: Different beam configurations obtained by setting the target position and magnetic horn current. The protons on target indicate the amount of data used for each configuration in this work.

\begin{tabular}{|c|c|c|c|}
\hline $\begin{array}{c}\text { Beam } \\
\text { Configuration }\end{array}$ & $\begin{array}{c}\text { Target } \\
\text { Position }(\mathrm{cm})\end{array}$ & $\begin{array}{c}\text { Horn } \\
\text { Current }(\mathrm{kA})\end{array}$ & $\begin{array}{c}\text { Protons On } \\
\text { Target } \times 10^{18}\end{array}$ \\
\hline \hline L010z170i & 10 & -170 & 1.44 \\
\hline L010z185i & 10 & -185 & 18.1 \\
\hline L010z200i & 10 & -200 & 1.35 \\
\hline L100z200i & 100 & -200 & 1.13 \\
\hline L250z200i & 250 & -200 & 1.55 \\
\hline
\end{tabular}

repetition rate is every 1.9 seconds which yields approximately $4 \times 10^{20}$ protons on target per year. The resulting particles produced in the target are focused by two magnetic horns, which are double-parabolic magnetic lenses. The horns can produce a maximum $30 \mathrm{kG}$ toroidal magnetic field which focuses charged pions and kaons toward a $675 \mathrm{~m}$ long evacuated decay pipe.

In the decay pipe, the pions and kaons decay into muons and neutrinos. Surviving hadrons are measured in a hadron monitor, which is a ionization chamber, at the end of the decay pipe and range out their energy in a absorber which consists of a water-cooled aluminum core, next a layer of steel block, and an outer later of concrete. The muons range out in the rock after the absorber, but intensity measurements are made through the use of three muon monitors, which are also ionization chambers, housed in three alcoves in the rock. The resulting neutrinos easily penetrate the rock and travel to the Near detector and onward to the Far detector in the Soudan Mine, Minnesota. The beam composition is $93 \% \nu_{\mu}, 6 \% \bar{\nu}_{\mu}$, $1 \% \nu_{e}+\bar{\nu}_{e}$. A schematic of this entire process is illustrated in Figure 3.2.

A unique aspect about the NuMI beamline is that by varying the position of the target and the second magnetic horn, one can produce various neutrino energy spectra that are measured in the detectors. This is because pions and kaons of dif- 


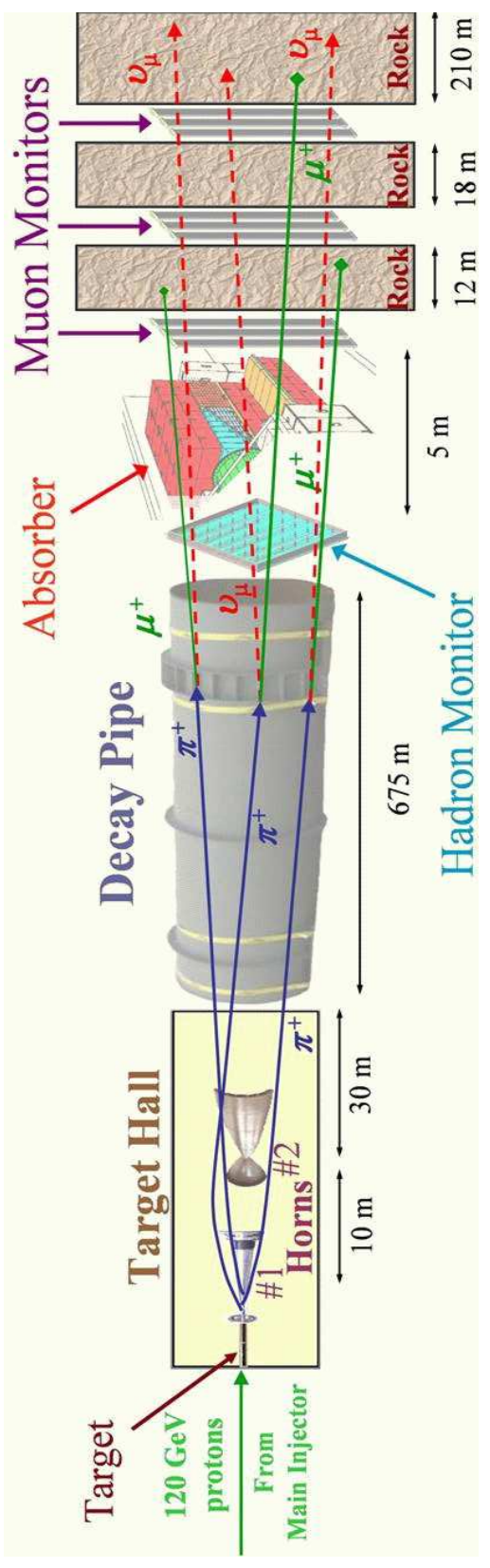

Figure 3.2: The layout of the NuMI neutrino beam production. Protons from the Main Injector hit a graphite target whose produced particles are focused down into the decay pipe. The pions and kaons decay into muons, which get absorbed downstream in rock, and neutrinos which continue onto the MINOS detectors. Figure produced by Robert Zwaska [85]. 
ferent momenta can be selected and focusing into the decay volume. An example of some different spectra are illustrated in Figure 3.3. This feature has been important in understanding beam systematics. Having multiple spectra gives one more information in which to understand our beam. These issues will be discussed later. The primary beam configuration has the target position at $10 \mathrm{~cm}$ and magnetic horn current kept at $-185 \mathrm{kA}$ due to the fact that the Far detector predicted spectrum will be peaked near $3 \mathrm{GeV}$, which maximizes MINOS' sensitivity to the oscillation parameters measured by Super-Kamiokande and K2K [84]. A Table outlining the various beam configurations used throughout this work are in Table 3.1.

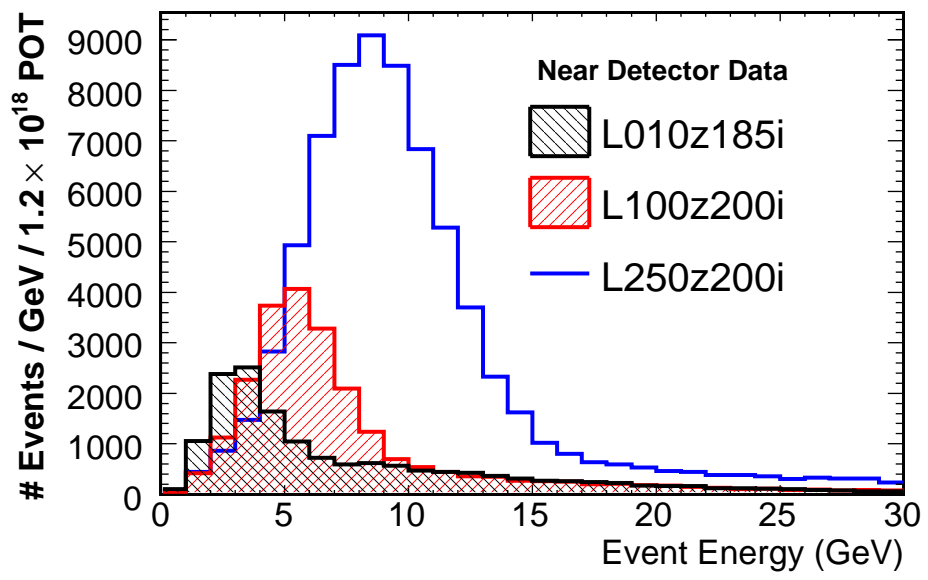

Figure 3.3: Three different beam configurations measured in the MINOS Near detector. The black line filled with top left to bottom right hashing has the target position at $10 \mathrm{~cm}$ and horn current at $185 \mathrm{kA}$, the red line with the bottom left to top right hashing has the target position at $100 \mathrm{~cm}$ and horn current at $200 \mathrm{kA}$, and the solid blue line has the target position at $250 \mathrm{~cm}$ and horn current at $200 \mathrm{kA}$. All three spectra are normalized to each other by total protons on target. 


\subsection{The MINOS Detectors}

\subsubsection{Particle Detection}

The MINOS detectors were designed to functionally have the same neutrino interaction detection schemes in order to minimize systematics between the two detectors. The basic unit of each detector is known as a "plane". Each detector plane is composed of $1 \mathrm{~cm}$ thick plastic scintillator of polystyrene, followed immediately by a $2.54 \mathrm{~cm}$ thick steel plane and an air gap for a total pitch of $5.94 \mathrm{~cm}$.

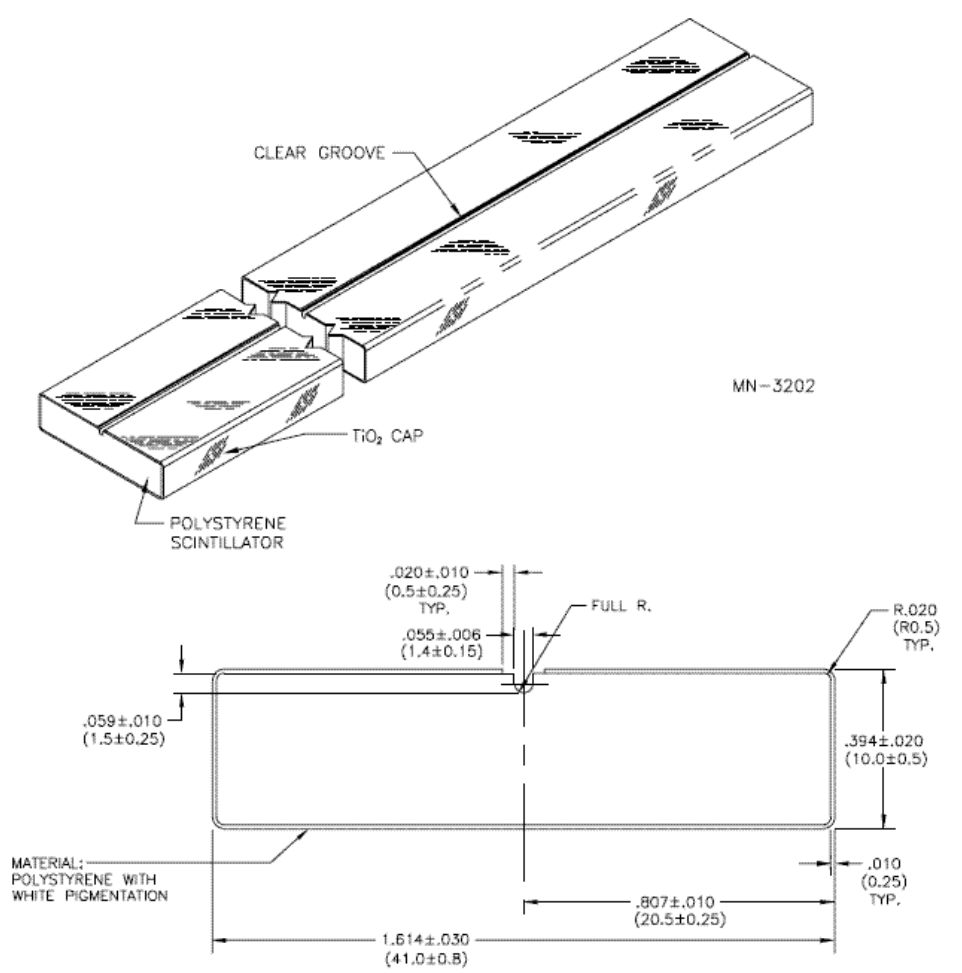

Figure 3.4: A diagram of a MINOS scintillator strip

Most of the mass of each detector is steel while the scintillator is the active detector element. Charged particles passing through the detector produce scintillation light which is captured on wavelength shifting fibers and read out by multi-anode 


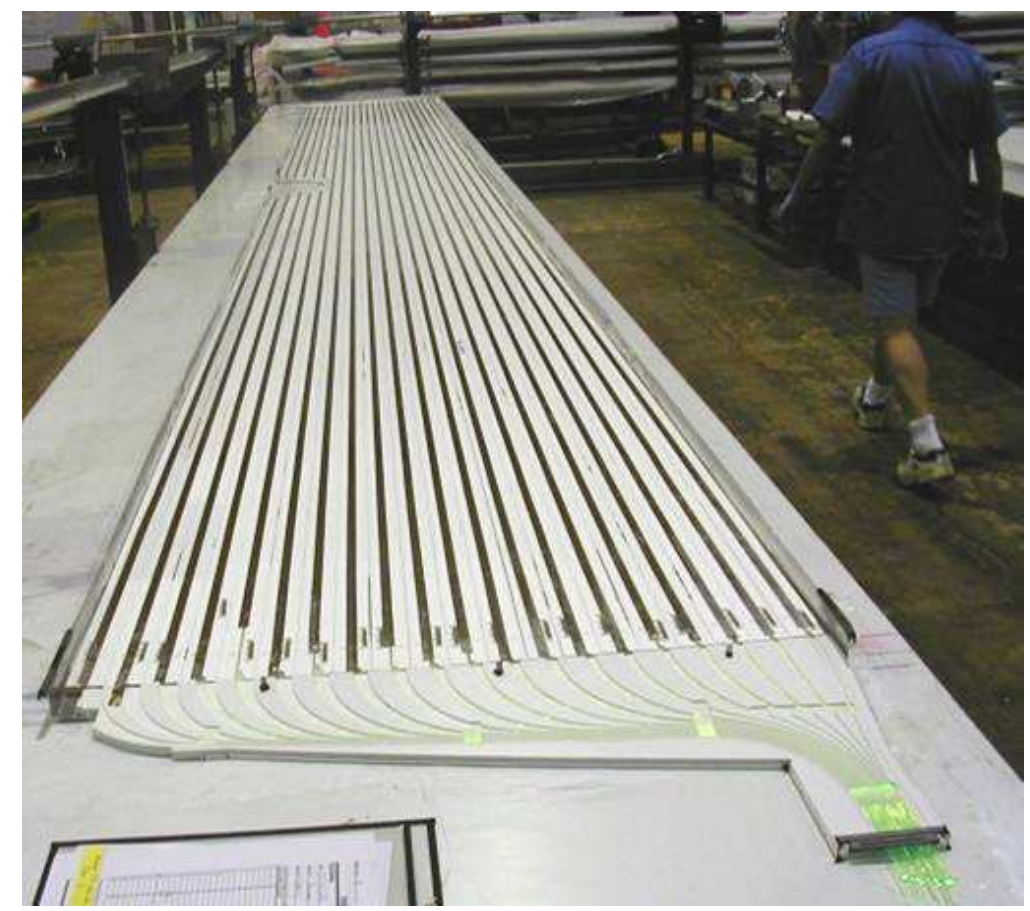

Figure 3.5: A diagram of a MINOS scintillator module

photomultiplier tubes.

\subsubsection{Scintillator}

The actual scintillator planes are segmented into strips that are $4.1 \mathrm{~cm}$ in width which are each read out by optical fibers to a multi-anode photomultiplier tube. The $4.1 \mathrm{~cm}$ wide scintillator strips are packaged into modules of 20 or 28 strips each. The end pieces of each module extend beyond the edge of the steel plane and contain fiber optics connections. Every other plane's scintillator strips are rotated by 90 degrees with respect to the previous plane in order to allow $(x, y)$ coordinates and thus three dimensional reconstruction of events. A diagram of a MINOS strip is shown in Figure 3.4 and the packaging of these strips into a module is shown in Figure 3.5. 
The scintillator strips are composed of Dow STYRON 664 polystyrene doped with PPO (1\% by weight) and POPOP ( $0.03 \%$ by weight) fluors and were produced by Itasca Plastics. These strips were surrounded by a co-extruded reflective layer of $85 \%$ polystyrene and $15 \% \mathrm{TiO}_{2}$ by weight. A $1.2 \mathrm{~mm}$ diameter wavelength shifting fiber is glued into each scintillator strip. A $2.0 \mathrm{~mm}$ deep groove is co-extruded along the length of each strip for this purpose. These grooves were then covered with aluminized mylar to maximize light collection.

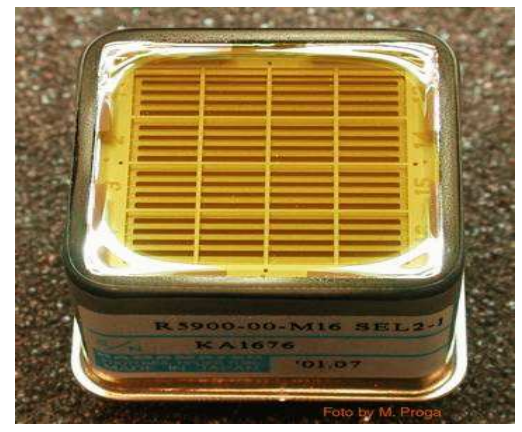

(a) Far Detector M16

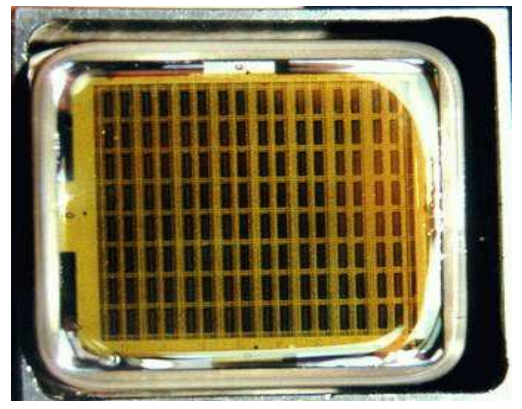

(b) Near Detector M64

Figure 3.6: The left picture is a Far detector M16 Hamamatsu photomultiplier tube, and the right is the Near detector M64 photomultiplier tube.

\subsubsection{Photomultiplier Tubes}

MINOS utilizes multi-anode photomultiplier tubes to convert scintillation light into an electrical signal to be further processed by the data acquisition system. We use Hamamatsu model R5900-M16 (M16) in the Far detector and model R5900-M64 (M64) in the Near detector. This corresponds to 16 and 64 pixels respectively and each pixel can have up to eight fibers from eight different scintillator strips. This allows MINOS to economically read out information from thousands of individual scintillator strips. The photomultiplier tubes were extensively tested and characterized $[86,87]$ on dedicated test stands. 


\subsubsection{Steel and Magnetic Field}

The steel of the MINOS detectors was produced by Olympic Steel Co., and was produced to have a very small Carbon content to increase magnetic permeability. In addition, another requirement was low radioactivity to keep the noise rate low in the detectors.

The Near detector has a toroidal magnetic field coil. The coil has 48 turns which breaks down into $18 \mathrm{~m}$ long central and return sections. The coil conductors are 1.5 inch by 1.1 inch aluminum with a central cooling channel for low conductivity water. The 40000 Amp-turn coil was designed to produce a toroidal magnetic field for muon momentum measurements. The average toroidal field at a radius of $1 \mathrm{~m}$ is $1.2 \mathrm{~T}$.

The Far Detector also has a magnetic field to allow curvature measurements of muon momentum. The Far Detector uses a water cooled coil with a total of $15 \mathrm{kA}$ turns for each supermodule. The average toroidal field, at a radius of $2 \mathrm{~m}$ is $1.5 \mathrm{~T}$.

\subsection{The MINOS Spectrometers}

\subsubsection{Near Detector}

The Near detector's purpose is to measure the energy spectrum of neutrinos before they have a chance to oscillate. Using this information, one can make a prediction for what to expect at the Far detector in the absence of oscillations. Analysis to discover discrepancies between data and Monte Carlo will be undertaken with the Near detector. Understanding and accounting for these differences will minimize systematic errors in the Far detector.

The Near detector weighs 0.98 kton and contains 282 steel planes that hang on a rail support structure, of which 153 have scintillator modules. It is located 98 $m$ underground at Fermilab to minimize overhead backgrounds. Excluding the air 


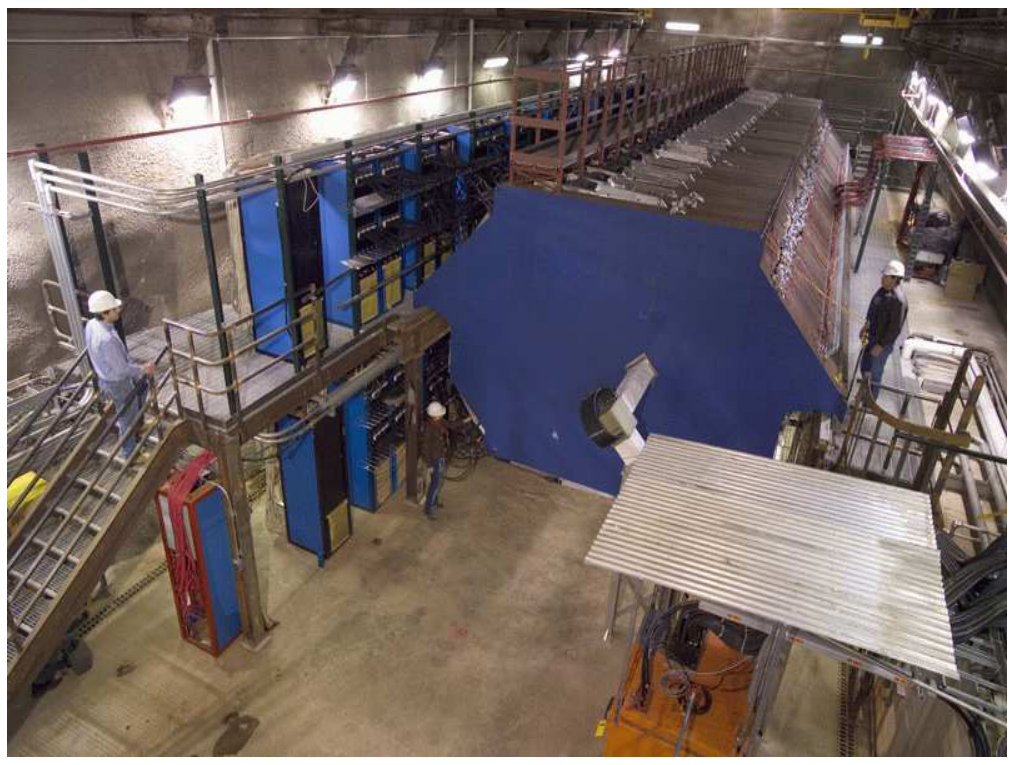

Figure 3.7: A picture of the Near Detector at Fermilab

gaps, the total length of the detector is $16.6 \mathrm{~m}$.

The Near detector is functionally divided into four sections, each having a different purpose. The first 20 planes are known as the veto section. Interactions with a vertex in this region are used to reject background events, predominantly rock muons which are muons that penetrate the front face of the detector that came from neutrinos that interact in the rock surrounding the detector. The following 40 planes are the target section. This area contains vertices of neutrino interactions that will be used in the various analyses. It is important for the interactions to be fiducially contained in a region where the energy does not leak out and thus bias our measurements. A fiducial volume requirement will be discussed when we elaborate on how one selects various neutrino interactions. The next 60 planes is the hadron shower section, which allows hadronic showers from neutrino interactions to fully develop. The last 160 planes is the muon spectrometer section, which measures the momenta of muons from charged current interactions. A picture of the Near 

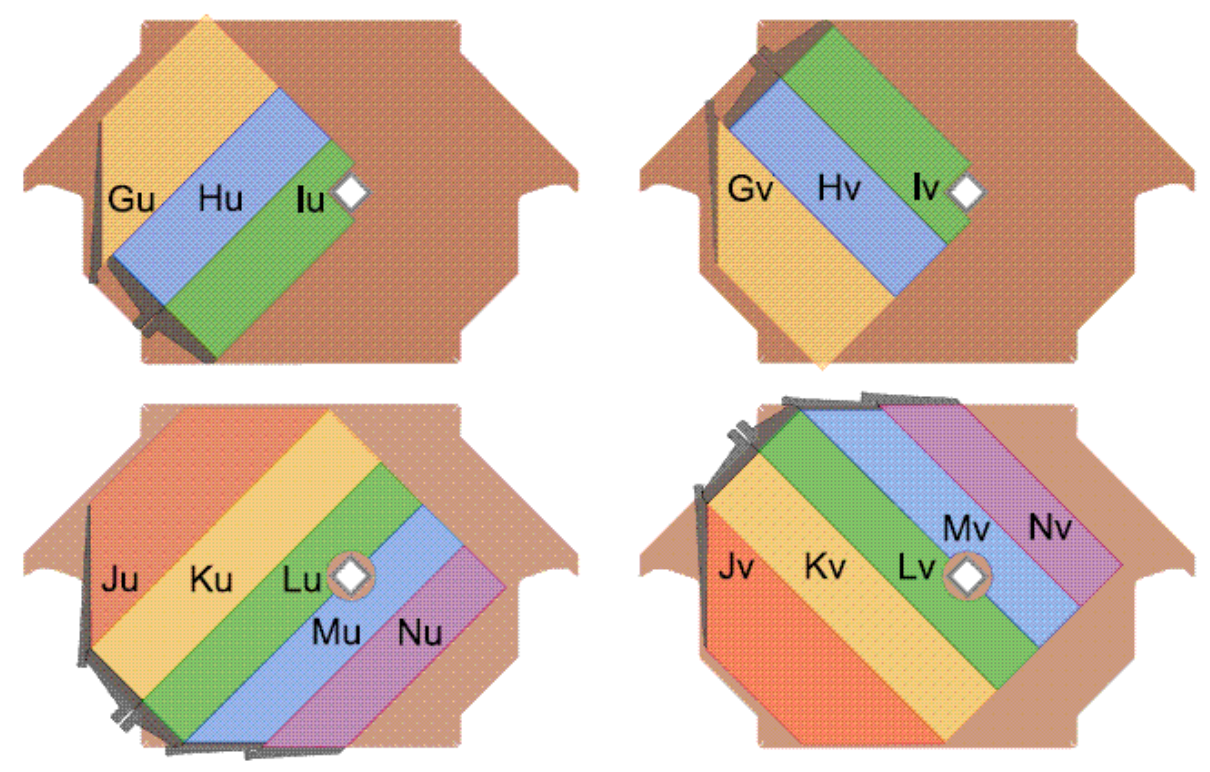

Figure 3.8: A layout of the Near detector planes. The top two plots show U and V partially instrumented planes, each with three scintillator modules. The bottom two plots show $\mathrm{U}$ and $\mathrm{V}$ fully instrumented planes, each with five scintillator modules.

detector is shown in Figure 3.7.

Each Near detector plane has scintillator modules on it, but the coverage of each plane is different. There are two types of planes in the detector design: fully instrumented and partially instrumented. Also as was mentioned earlier, every other plane has it's scintillator strips rotated by 90 degrees with respect to the previous plane to allow three dimensional reconstruction, these are each referred to as $\mathrm{U}$ and V planes respectively. This is illustrated by the Near detector plane layout in Figure 3.8 .

Most of the detector uses the partially instrumented planes, but every fifth plane in the detector is fully instrumented. The reason for this is that the neutrino beam interactions occur within a very small region and the fully instrumented planes allow MINOS to have better tracking ability for muons which exit the partially 
covered planes. Partially instrumented planes have a total of 64 scintillator strips while fully instrumented have 96 .

The Near detector has readout of each scintillator module coming only from one end, unlike the Far detector in which readout is from both ends. The event rate in the Near detector is very high and the beam intensities from the Main Injector can produce one or more events in the Near detector in each RF bucket during the spill. In order to reconstruct each event properly it is necessary to associate every event with a particular RF bucket. This requires very fast electronics, capable of digitizing at the $53 \mathrm{MHz}$ RF frequency from the Main Injector at Fermilab. Also, since the spill may last up to $\approx 10 \mu s$, it is desirable that there be no deadtime during the spill, so every neutrino event can be recorded. The Far detector has a lower event rate than the Near detector and it would not be advantageous to put fast electronics there. Since the Near detector has half as many channels and is only read out from one end, there was a significant cost advantage to having fast electronics only in the Near detector. Details on the Near detector electronics can be found in [88].

The fibers extending from the scintillator planes run to electronics racks where they are connected to a multi-anode photomultiplier tube. Each partially instrumented plane in the Near detector uses one photomultiplier tube, whereas two fully instrumented planes share one. By having two fully instrumented planes share one tube, one minimizes the amount of hardware, but an electrical summing technique is developed for the muon spectrometer region. The signal from four adjacent pixels is summed and read out by a single electronics channel. Since these adjacent pixels are not connected to adjacent strips it is possible to resolve the four-fold ambiguity in strip space. This is known as multiplexing. 


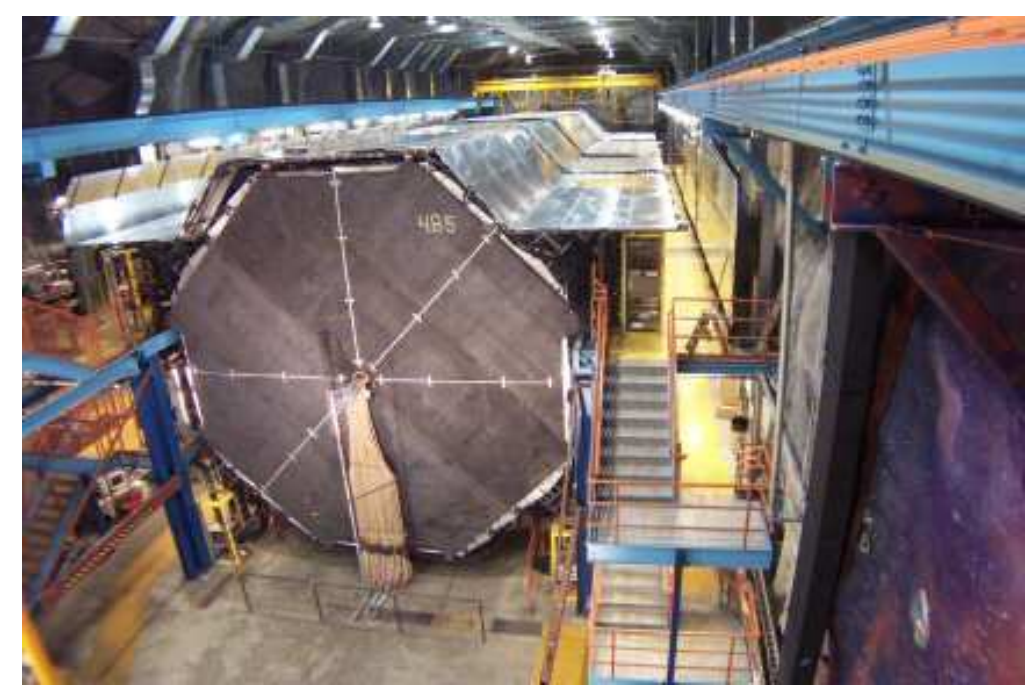

Figure 3.9: A picture of the Far Detector at Soudan Mine, Minnesota.

\subsubsection{Far Detector}

The main purpose of the MINOS Far detector is to measure the oscillated neutrino energy spectrum so that it can be compared with an unoscillated prediction from the Near detector. It is a 5.4 kton magnetized iron calorimeter located at $705 \mathrm{~m}$ underground in the Soudan Underground Laboratory in Soudan, MN. The Far detector is split into two supermodules, which are functionally identical. This was done so that the first half can immediately start taking atmospheric neutrino data. Each supermodule is $8 \mathrm{~m}$ in diameter and taken together have a length of $31 \mathrm{~m}$. There are a total of 486 steel planes, allowing 249 for the first and 237 for the second supermodule. There is a $1.5 \mathrm{~m}$ long gap between the supermodules to allow space for magnetic coils. A picture of the Far detector is shown in Figure 3.9.

Each scintillator plane is made up of 192 strips and as in the Near detector, the orientation of the strips alternates by ninety degrees to allow three dimensional reconstruction. Light is transported from both ends of each strip. These ends are coupled through clear fibers and to multipixel photomultiplier tubes. The Far 
detector photomultiplier tubes have 16 pixels and an example is shown in Figure 3.6. A layout of each scintillator plane is shown in Figure 3.10. Eight fibers from eight strips spaced roughly $1 \mathrm{~m}$ apart on a detector plane are coupled to each pixel. The resultant eight-fold ambiguity can be resolved in software by utilizing the fact that the exact arrangement of which fiber is coupled to which pixel is somewhat different at the two ends. Timing and pulse height information from adjoining planes will be used to provide an independent ambiguity resolution. This allows one to identify which strip was actually hit by a particle traversing the detector. The optical matching is done in steel boxes that house the photomultiplier tubes. Each box contains three tubes and reads out one side of two planes which corresponds to $2 \times 192$ strip ends.

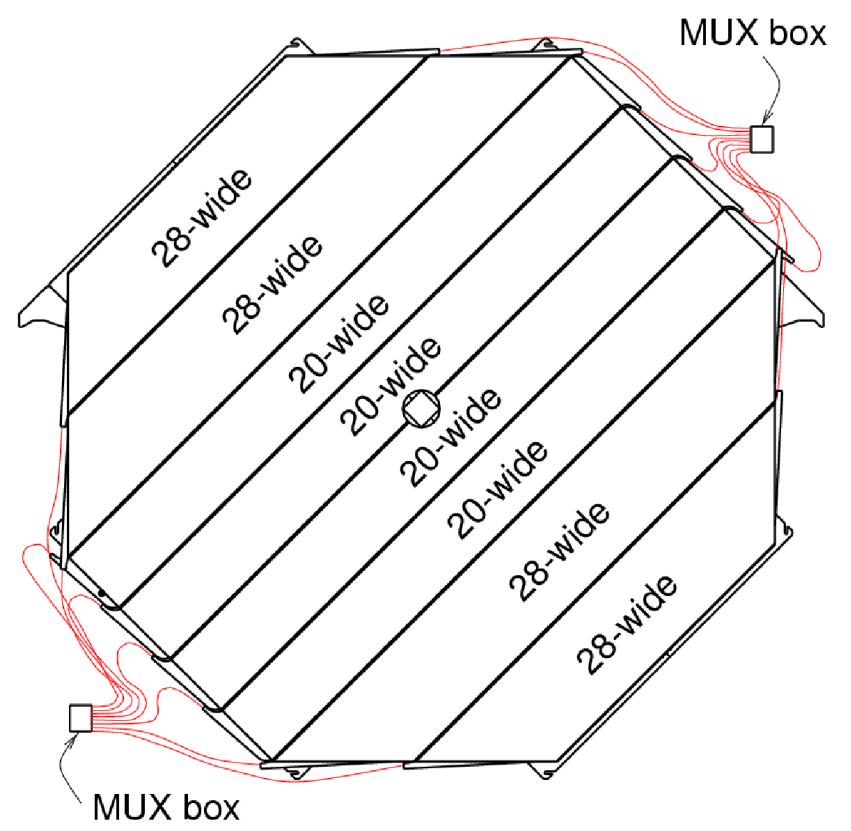

Figure 3.10: A layout of a Far detector plane and its corresponding scintillator modules. 
Due to spontaneous light emission rates in the wavelength shifting fibers MINOS has implemented a low level trigger [84] known as the "2/36 trigger". It requires coincidence of two dynode triggers in a 36 plane window. A higher level trigger is known as the "spill trigger". When a neutrino beam spill occurs, a signal from the beam extraction magnet at Fermilab is sent to the Near detector which is timestamped and sent to the Far detector which causes a continuous readout of a $100 \mu$ s window. This trigger is vital to eliminating backgrounds in the Far detector that mimic low energy neutral current events. Chapter 8 describes the selection of neutrinos in the Far detector and the elimination of this background.

The Far detector also has a veto shield positioned overhead and on the cavern walls on either side of the Far detector. This veto shield has been crucial for the atmospheric neutrino analysis to eliminate cosmic ray backgrounds.

\subsubsection{Calibration Detector}

The Calibration Detector (CalDet) was a 12 ton model of the MINOS Near and Far detectors that was exposed to test beams in the CERN PS East Hall during several runs in 2001-2004 [89]. The data was collected to determine the response of the MINOS detectors to electrons, muons and hadrons so that a neutrinos's energy can be accurately measured $[90,91]$. It also provided a benchmark for comparison and tuning of Monte Carlo simulations. Also, CalDet helped compare the different electronics used in the Near and Far Detectors for systematic studies [92].

Since the ultimate MINOS observable is the visible energy of neutrino interactions, CalDet was instrumental in ensuring that energies are properly measured and calibrated. Through analysis of CalDet data, the measured hadronic shower resolution for the MINOS detectors was found to be [90]

$$
\frac{(56.6 \pm 0.6) \%}{\sqrt{E[\mathrm{GeV}]}} \oplus(4.2 \pm 1.4) \% \quad \text { (protons) }
$$




$$
\frac{(56.1 \pm 0.3) \%}{\sqrt{E[\mathrm{GeV}]}} \oplus(2.1 \pm 1.5) \% \quad \text { (pions) }
$$

and the electromagnetic shower resolution was measured to be [91]

$$
\frac{(21.42 \pm 0.06) \%}{\sqrt{E[\mathrm{GeV}]}} \oplus(4.1 \pm 0.2) \% \quad \text { (electrons) }
$$

\subsection{Light Injection System}

The MINOS Light Injection (LI) system measures and compensates for the individual gains of each one of the optical readout channels, identifying drifts in response on a channel by channel basis and linearizing the response of photomultiplier tubes and electronics. The system uses pulsed UV LEDs to inject light in the detector's optical path and then compares the output to an independent measure of the light injected.

When the LI system is operating in the Far detector, flashes of light are occasionally recorded during beam spills. These flashes can be reconstructed as events and must be eliminated as a source of background. This issue is discussed in Chapter 8 .

\subsection{Summary}

We have outlined the important components of the MINOS detection system, including the scintillator technology, photomultiplier tubes, and the detectors themselves. In the next chapter we discuss the software used to reconstruct neutrino events from signals in the detectors. 


\section{Chapter 4}

\section{Event Reconstruction}

Measurement of physical quantities associated with neutrino interactions requires reconstruction of these quantities from individual scintillator signals in the MINOS detectors. Also, simulations are paramount to our understanding of the detectors and data. A brief introduction to the Monte Carlo is initially discussed followed by a concise description of the reconstruction software. Substantially more time will be spent discussing the event isolation algorithm where the author has most significantly contributed.

\subsection{MINOS Monte Carlo}

The event generation is done through the NEUGEN neutrino event generator [93]. NEUGEN is a neutrino event generator and cross section library that simulates neutrino-nucleus interactions over the energy range $100 \mathrm{MeV}-100 \mathrm{GeV}$. The primary NEUGEN interaction models of importance for this analysis are quasi-elastic, resonance production, and deep-inelastic scattering. Quasi-elastic scattering is treated with a Fermi Gas model of Pauli blocking. The resonance model is from ReinSeghal [94] and the DIS model is a modified leading order QCD model with new 
scaling variables to better describe data in the DIS/resonance overlap region [95].

The Monte Carlo has a number of parameters associated with it that can be used to tune our simulation to the data. In Chapter 8 systematic studies are performed where the simulation parameters are changed by their $\pm 1 \sigma$ values to see the effect on our measurement of the oscillation parameters.

\subsection{Reconstruction Software}

MINOS has a well defined reconstruction software chain to extract physics quantities about individual neutrino interactions. We now discuss the main sequences of the chain starting with the output from the data acquisition system which is out of the scope of this work.

\subsubsection{First Stage of Signal Processing}

A "digit" is defined as any individually recorded signal in any channel in either MINOS detectors. The software for this element of the reconstruction is quite simple, in that it records the plane and strip in which the signal occurred, in addition to the time of occurrence and pulse height in ADC counts.

\subsubsection{Second Stage of Signal Processing}

Because multiple digits can occur in a physical scintillator strip in either detector, they must be separated to account for what could be hits from different neutrino interactions. The individual digits are sorted by plane, strip position, and time. For each physical strip in the detector the number of digits is stepped through in time to look for large time gaps between individual digits. If the time difference between a digit in question and the next digit is less than $60 \mathrm{~ns}$, one continues to step through the rest of the digits. Once the next digit is more than $60 \mathrm{~ns}$ away from the previous digit, a "strip" is formed and the next digit begins the new strip. The total length 


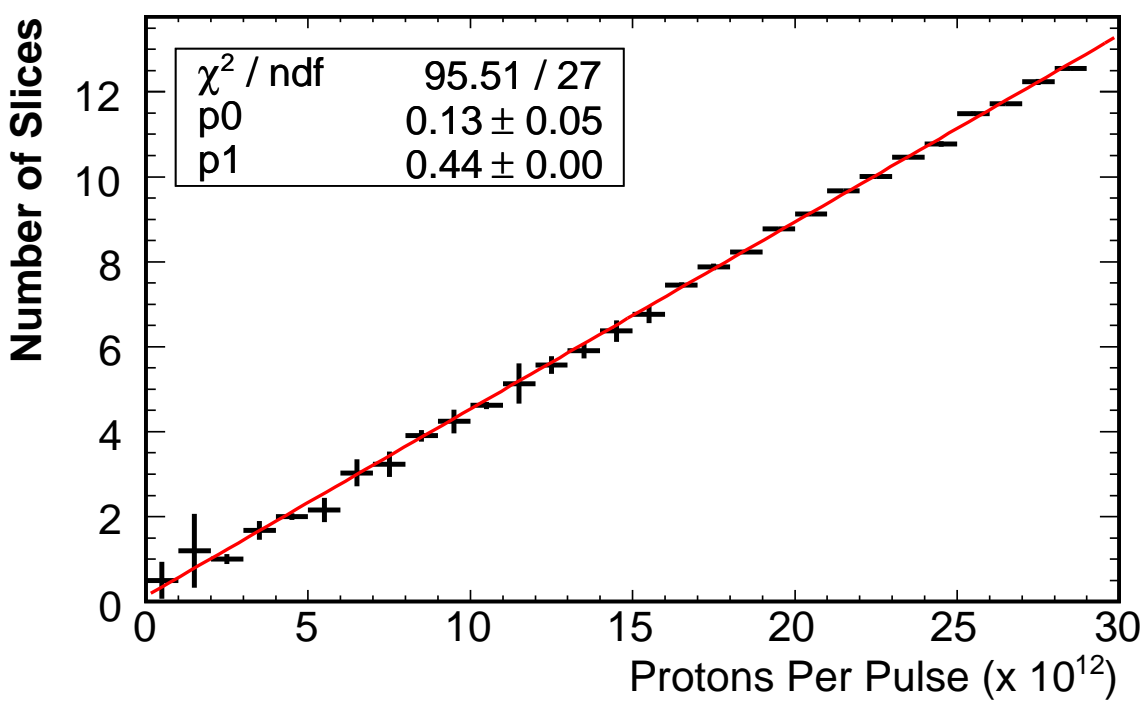

Figure 4.1: A plot of beam intensity in units of protons per pulse versus the average number of reconstructed slices. The histogram is fit with a straight line.

in time of all the digits in any strip cannot be more than $120 \mathrm{~ns}$ in order to ensure the total time duration is sensible.

\subsubsection{Event Isolation}

With the construction of the NuMI beamline, Fermilab has the world's most intense neutrino beam. The number of neutrinos reaching the MINOS detectors will depend on the number of protons on target in a single spill which is typically at least $2.5 \times 10^{13}$ protons per pulse $(\mathrm{ppp})$, and has so far achieved a record of $4.0 \times 10^{13}$ ppp. With the difficulty of neutrino physics in the past being statistics, MINOS is certainly an achievement. Coupled to this benefit is the logistical problems associated with so many neutrino interactions in the Near detector. With such a high number of neutrinos interacting in the MINOS Near detector, it is essential to isolate the separate neutrino interactions as best as one can in order to pass them down the reconstruction chain to shower finding, tracking, and finally event formation. This 
algorithm, developed and tested by the author is called the Slicer Algorithm, and the application of this algorithm is called "slicing". The Slicer produces "slices", which are just a collection of strips grouped together.

Figure 4.1 is a plot of the average number of slices reconstructed as a function of beam intensity. Notice the linear relationship between the number of slices and beam intensity. The main challenge of the slicing algorithm is to verify that the actual process of slicing does not bias our energy spectrum in the Near detector. The way to do this is to compare reconstructed physics quantities as a function of intensity. MINOS has taken data at lower intensities to compare to data at our nominal higher intensities. At lower intensities fewer interactions and less activity are expected in the detector. This makes for a cleaner reconstruction environment since the slicing algorithm has fewer interactions to separate. Since there is less activity we do expect differences between spectra at lower and higher intensities. This is not an issue as long as we can show that these differences are similar between our data and Monte Carlo. We undertook detailed intensity studies in Chapter 6 in order to show that the slicer does not bias comparisons between data and Monte Carlo.

To illustrate the problem at hand with slicing, the transverse $(u), z$, and $\mathrm{t}$ coordinates of all the strips in a typical Near detector beam spill with sixteen interactions is shown in Figure 4.2. The top plot is the spatial coordinates, while the bottom is the timing distribution of strips. It is apparent that reconstruction must separate each interaction before any physical quantities can be extracted. The spatial positions by themselves are not enough to accomplish this task. The timing distribution of all the strips, as shown in the bottom plot has peaks in time that correspond to isolated activity in the detector. Appendix A shows the results of slicing the particular beam spill shown in Figure 4.2 with some detail on the individual slices. 

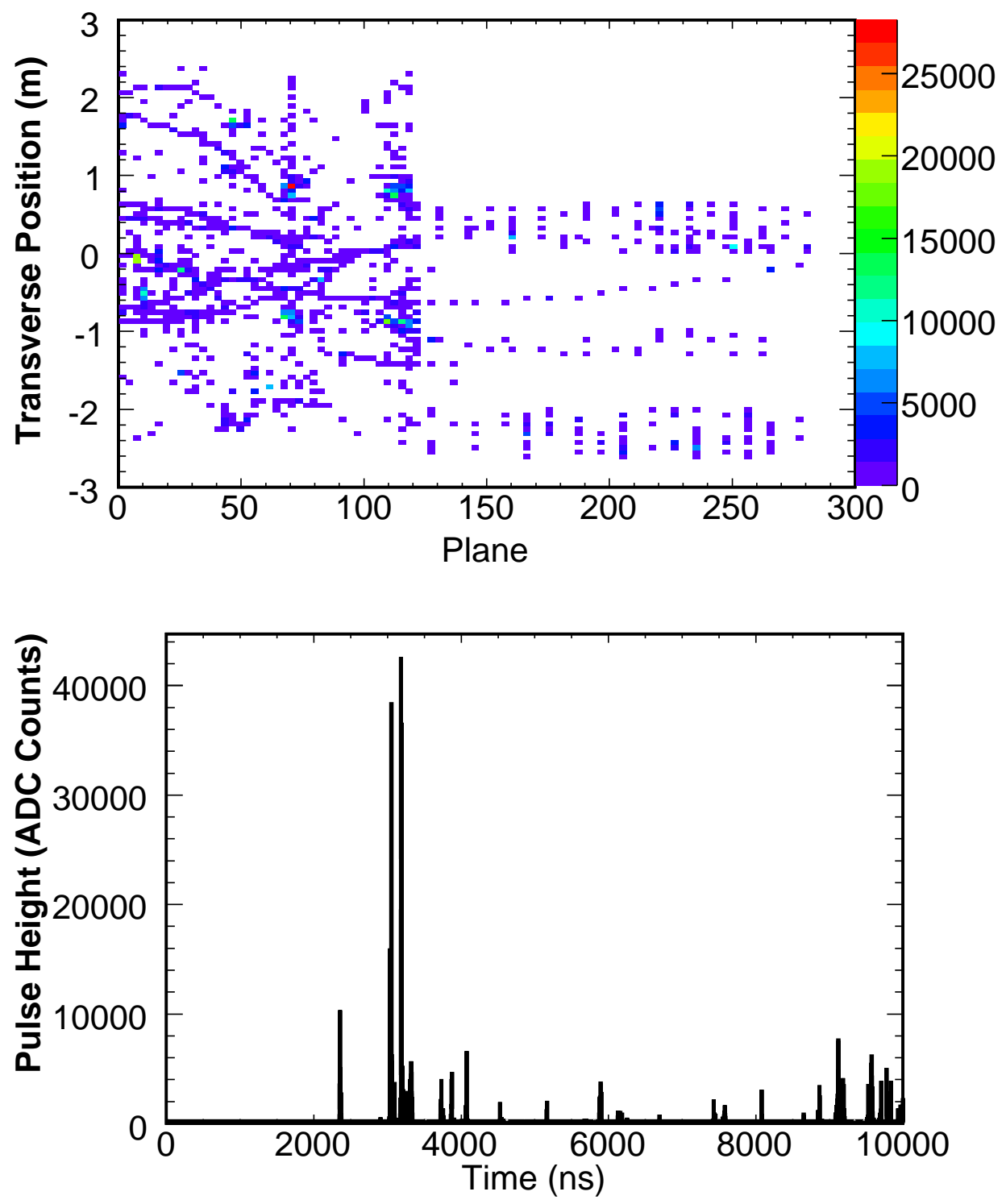

Figure 4.2: The top plot is a typical Near detector spill with an intensity of $2.42 \times 10^{13}$ protons per pulse. Each point is a individual strip in the detector and the color denotes pulse height in ADC counts. The z-axis is the plane number and the y-axis is the transverse position $(\mathrm{m})$ in the detector. The bottom plot is the pulse height weighted timing distribution of all the strip hits in the detector. Each spike corresponds to some activity or interaction. 


\section{First Stage of Slicing}

The initial stage of slicing deals only with strips that have a pulse height greater than 2.0 photoelectrons and are not in the spectrometer section of the Near detector. The pulse height requirement removes strips from the timing distribution of which $>99 \%$ are detector noise, cross-talk from photomultiplier tubes, and low pulse height strips from neutrino interactions, which pollute the timing distribution. Removing these strips allows the peaks in the timing distribution to be more distinctly separated in time. The reason for not initially including spectrometer strips is because interactions wholly contained in the spectrometer are not reconstructed properly due to only having one in five planes instrumented. Figure 4.3 shows the effect of these initial cuts on the timing distribution. The black histogram is a distribution of all strips in a certain time window and in red the strips that had a pulse height of at least 2 photoelectrons and not in the spectrometer.

\section{Second Stage of Slicing}

The timing distribution of strips with a pulse height of at least 2 photoelectrons and not in the spectrometer is used to step through the strips as they are ordered in time. This stage starts at the first strip in the timing distribution and requires that the next strip in time be no more than 20 ns away. If the next strip is more than this, a new slice is created and the process is started again with the current hit. After this is completed, the initial list of slices is created.

\section{Third Stage of Slicing}

The next phase of slicing deal with adding strips into slices that came from the spectrometer section and the low pulse height hits ( $<2$ photoelectrons) that were dropped by the first stage. The top plot in Figure 4.4 shows the z-position in plane number versus timing in nanoseconds. The black points are strips from the initial 


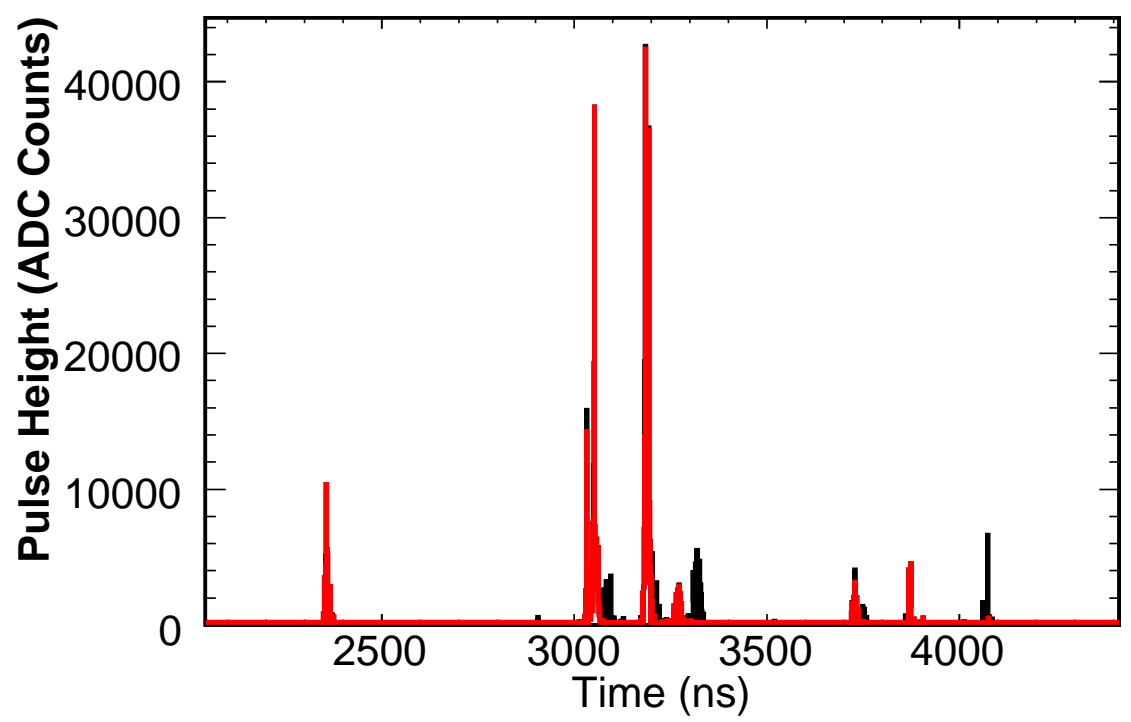

Figure 4.3: The timing distribution of a selection of hits in a $10 \mu$ s neutrino spill. The black hits are all hits that occurred in the time interval, and the red is the same distribution with strips removed that had a pulse height less than 2 photoelectrons and in the spectrometer section.

list of slices. The red points at longitudinal positions greater than plane 121, which is the border between the calorimeter and spectrometer sections of the Near detector, are strips that need to be added into the existing slices. The red hits that penetrate deep into the spectrometer are muons from charged current $\nu_{\mu}$ interactions. If a strip in the spectrometer is within $-40<t_{\text {slicestart }}<100 \mathrm{~ns}$ of the start time of a slice, it is added into that slice. After this is complete, the list of slices is updated with these new strips from the spectrometer.

Strips with a pulse height of less than 2 photoelectrons are then added into the appropriate slice in time. In order to accomplish this, a list of the start times for each of the slices created in the second stage is constructed. This is simply defined as the time of the earliest strip in the slice. If a low pulse height hit happens to be within the range of $\left[t_{\text {slicestart }}, t_{\text {sliceend }}\right]$ it is added to the slice. The strips added 

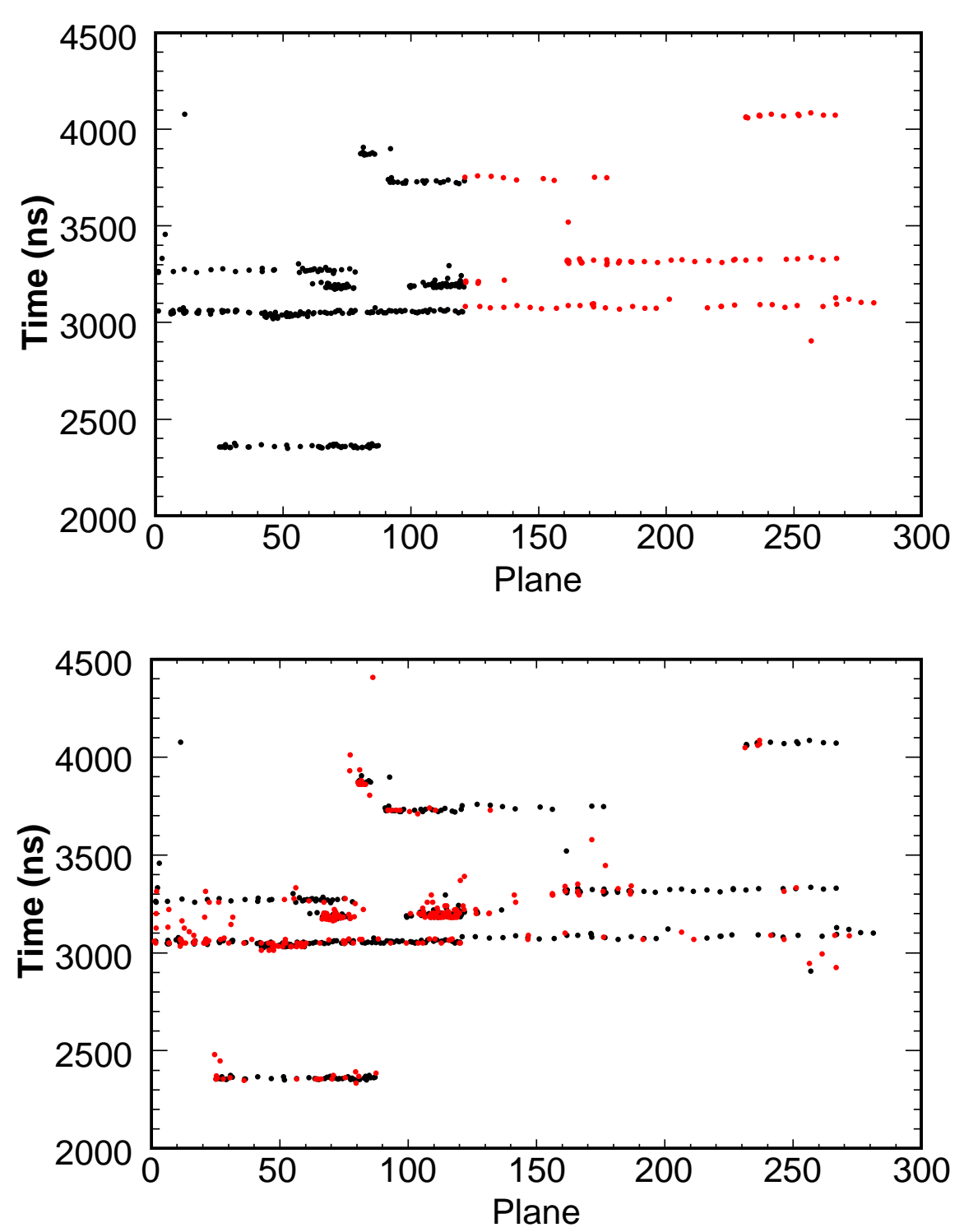

Figure 4.4: Both plots show the distribution of hits in the detector with the $\mathrm{x}$-axis being the $\mathrm{Z}$ position (in Planes Number) and the $\mathrm{y}$-axis is the time of the hit in nanoseconds. The top plot shows hits in the spectrometer section in red that are added into existing slices in black and the bottom plots shows the remaining low energy ( $<2$ photoelectrons) hits in red added into the existing slices in black. 
in this way are shown in the bottom plot of Figure 4.4. The black hits are hits in existing slices and the red are low pulse height strips that are added back in.

\section{Fourth Stage of Slicing}

The last phase of slicing simply eliminates slices that have a total pulse height $<2000$ ADC counts. This energy is roughly equal to about $200 \mathrm{MeV}$, of which anything with less energy will be poorly reconstructed. For more detail on all the slices in this example see Appendix A.

\section{Slicing Figure of Merit Variables}

Initial validation of the standard reconstruction slicer algorithm was done by the use of figure of merit variables. There are two variables that help define a measure of the performance of the slicer algorithm. They are called the completeness and the purity for a slice.

Using Monte Carlo, for every slice, one loops over all the strips in a slice to see what fraction of the strips came from what neutrino, which we get from truth information from the Monte Carlo. When the neutrino that deposited the most energy in the slice is found we define it to be the "Most Likely Neutrino" (MLN). For a particular "Most Likely Neutrino" one can find from truth, the total amount of energy deposited in the entire detector $E_{M L N, t o t a l}$, and also one can calculate how

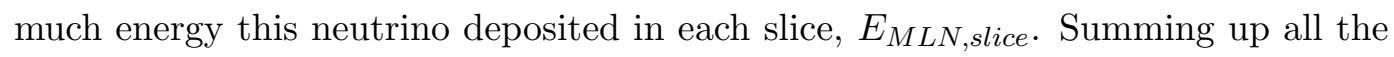
total energy in the slice, we define $E_{\text {slice,total }}$. Using these definitions, we can define 


$$
\begin{gathered}
\text { Completeness }=\frac{E_{M L N, \text { slice }}}{E_{M L N, \text { total }}} \\
\text { Purity }=\frac{E_{M L N, \text { slice }}}{E_{\text {slice }, \text { total }}}
\end{gathered}
$$

If a slice happens to be $90 \%$ complete and $80 \%$ pure, this means that $90 \%$ of a slice's energy belongs to a particular neutrino and that neutrino has $10 \%$ of its energy elsewhere in the detector. The fact that it is $80 \%$ pure means that $20 \%$ of the slice's energy came from other neutrinos. The ultimate goal is for all slices to be $100 \%$ complete and pure. We show the distribution of completeness and purity from Monte Carlo in Figure 4.5. The top plot shows that a majority of slices are of very high completeness, yet there exists slicing errors that cause a low completeness portion of the spectrum. Removing low completeness events is discussed in our selection of neutrino interactions in Chapter 5 where application of this removal criteria lowers the number of low completeness events from $3.6 \%$ to $0.6 \%$. The bottom plot shows slices of a high purity, although there exists some pollution from spurious hits and overlaps of more than one event in time and spatial position.

As discussed earlier, one of the advantages of having different proton intensities for each beam configuration is that one can try to understand the Slicer's performance as a function of beam intensity. More importantly, by understanding the functionality of beam intensity, one can understand any bias the slicer may introduce. We discuss the results of these studies in Chapter 6 and show that any effect that slicing may have on the data is reproduced in Monte Carlo. 

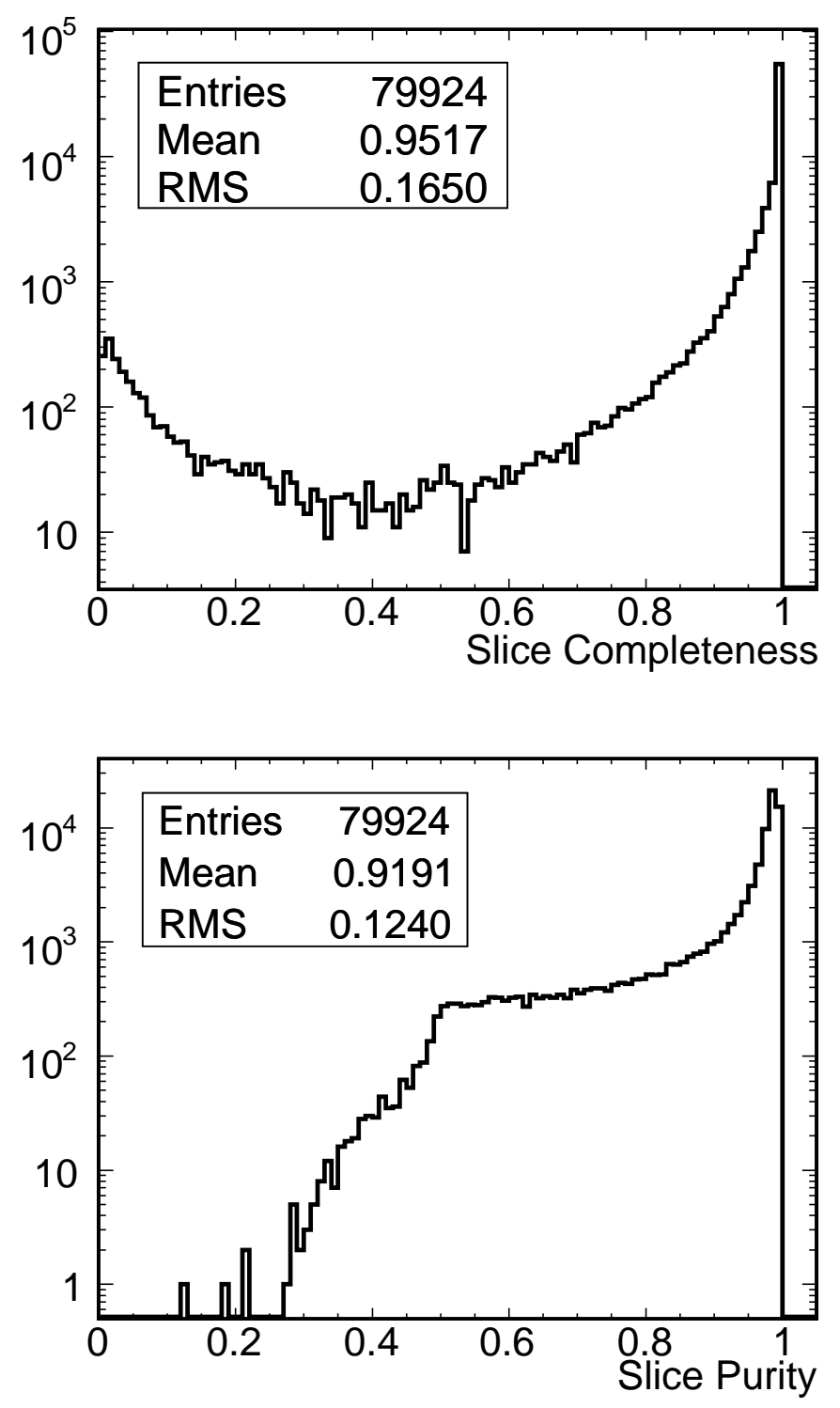

Figure 4.5: The top plot is the completeness of slices, while the bottom plot is the purity of all slices from application of the Slicer algorithm in the Near detector. This information can only be obtained from Monte Carlo. 


\section{Justification of Slicing Time Step}

Timing is the most useful quantity in slicing and since the algorithm initially steps through the timing distribution to find slices, the time step of $20 \mathrm{~ns}$ must be justified. Since neutrino interactions last on the order of $\approx 100 \mathrm{~ns}$, several tests were performed for different time steps of 20,60, 100, and 140 ns on Monte Carlo simulation. There were two figures of merit for this study. The first is the overall efficiency of finding charged current and neutral current events. The second is the amount of low completeness events in the charged current and neutral current spectra. The principle here is to maximize the number of high completeness events (Completeness $>50 \%$ ) and minimize the number of low completeness events (Completenes $<50 \%$ ).

Figure 4.6 shows the resulting charged current and neutral current energy spectra for timing steps of 20,60,100, and $140 \mathrm{~ns}$. Also the dashed line is the low completeness component of the energy spectra. A detailed zoom of the low completeness component is plotted in Figure 4.7. The y-axis label is less than one because the plots were each normalized by 1 divided by the total protons on target. One can conclude from these figures that 20 ns allows the highest efficiency with the smallest increase in low completeness events. The reason for this is because longer time steps increases the probability that more than one neutrino interaction will be in a slice. Downstream reconstruction software is not as efficient in separating multiple interactions, hence we have a loss in the number of charged current and neutral current events. 

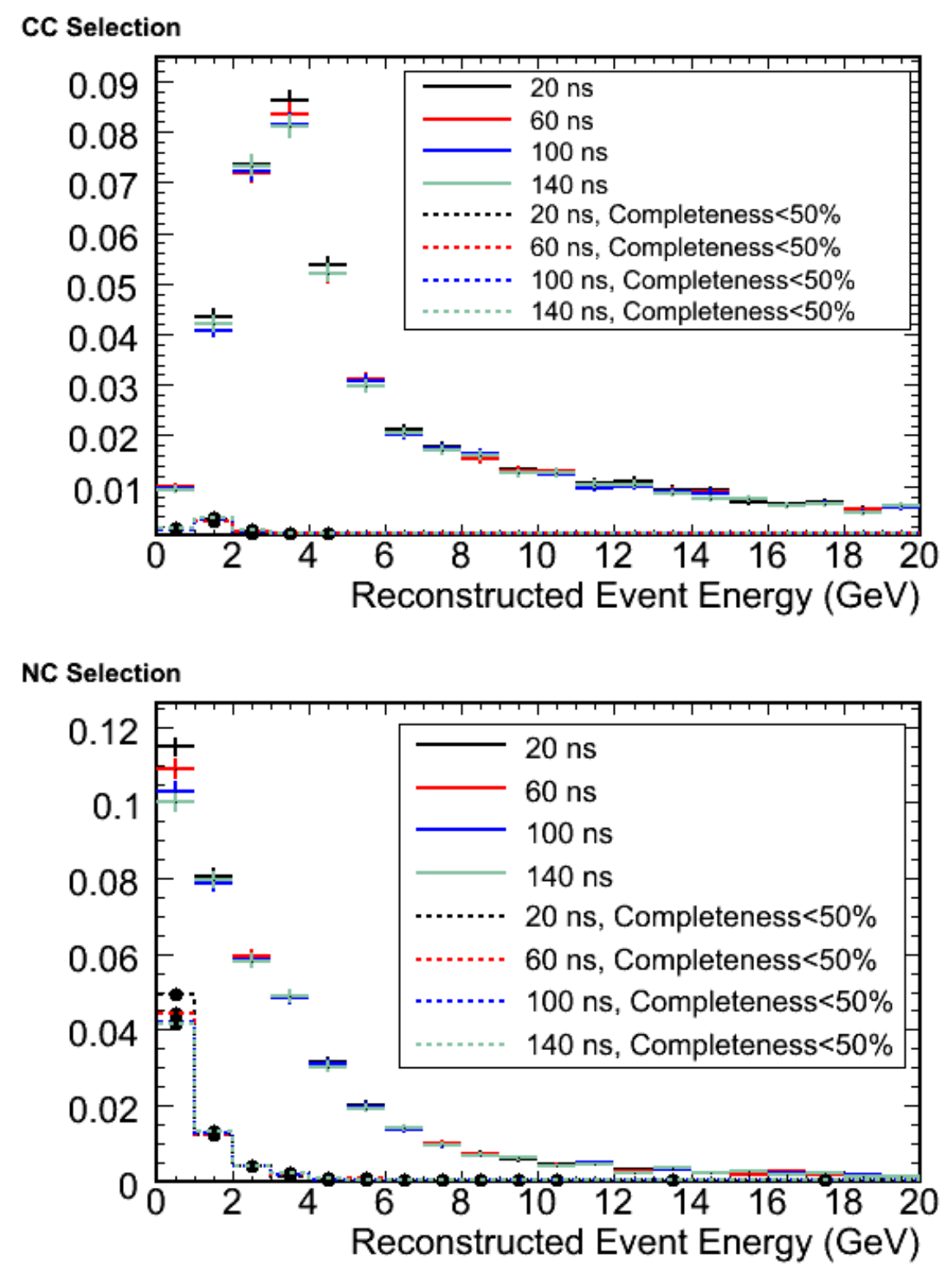

Figure 4.6: The charged current and neutral current energy spectra for different timing steps in the Slicing algorithm. The solid lines are the entire energy spectrum, while the dashed lines are the component of events that have a completeness less than $50 \%$. 

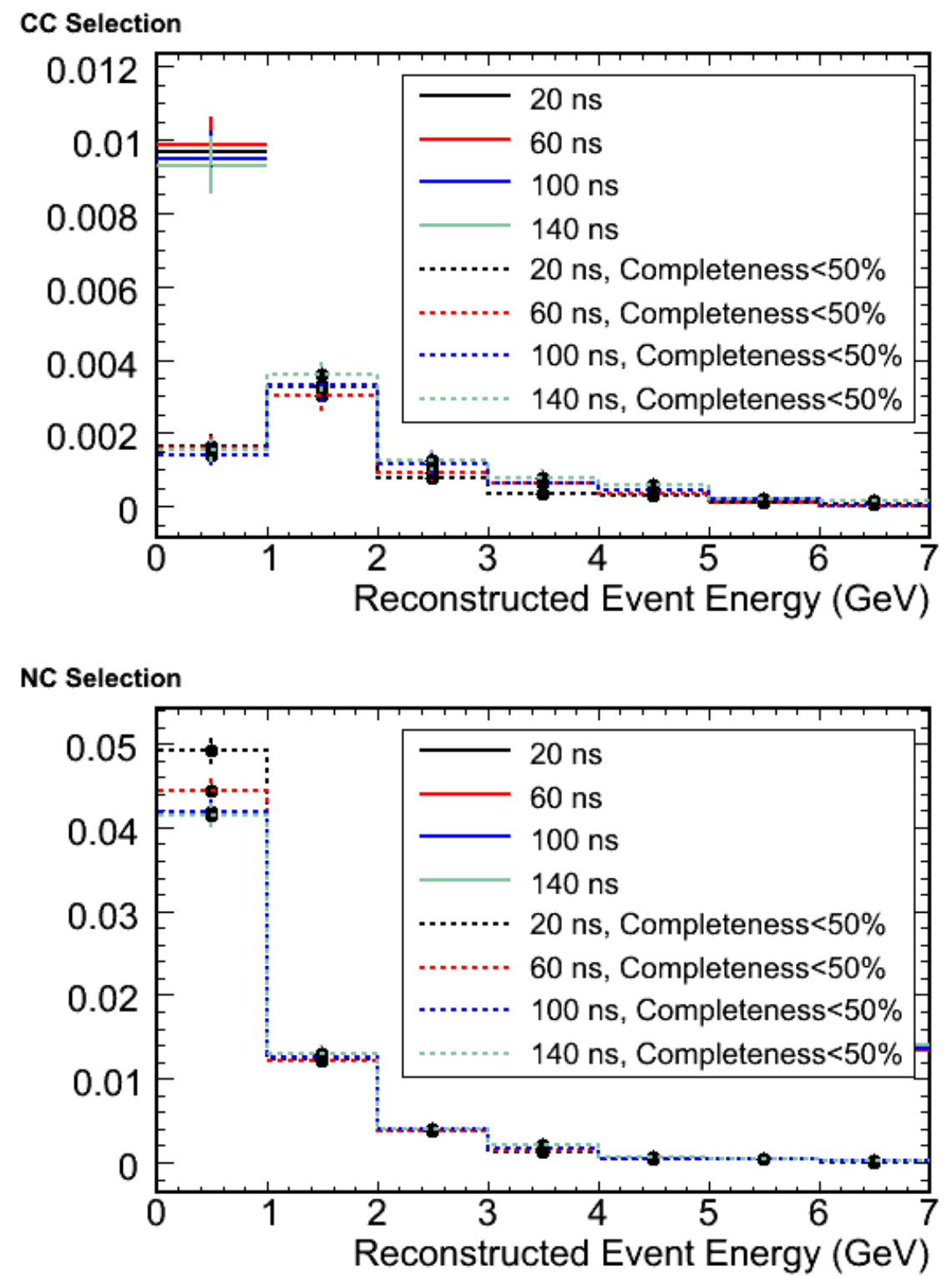

Figure 4.7: A zoom in on the low completeness portion of the charged current and neutral current energy spectra for different time steps in the Slicing algorithm. 


\subsubsection{Cluster Formation}

After we have a final list of slices, we try to identify "clusters" in each slice. A cluster is a object that consists of strips that are spatially adjacent and are defined to be two dimensional objects. These are later used to form three dimensional showers.

This algorithm constructs a neighbor map, which holds for each strip in each slice, the number of neighbor strips within $60 \mathrm{~ns}$ and no more than 3 physical strips away in length. After this point, one applies the algorithm to U and V (orthogonal) views separately, hence the two dimensional nature of a cluster. Topological criteria are then applied to all the cluster in each view combining them if they lie within some constraints.

\subsubsection{Shower Formation}

The shower formation stage consists of two parts. The first part known as the subshower formation uses the two dimensional clusters from the previous algorithm to form sub-showers, before they are passed onto the final three dimensional shower forming stage. This initial subshower formation is used to exploit the pulse height structure of the shower and to provide access to this reconstructed information at the event level. The second part combines clusters to reconstruct the hadronic (or electromagnetic) shower and its energy.

\subsubsection{Track Formation}

All the strips and clusters in a slice are passed to the track finder. First small tracklike clusters are attempted to be joined to construct a track. A Hough-transform is used to map $(\mathrm{u}, \mathrm{z})$ and $(\mathrm{v}, \mathrm{z})$ space into a $(\mathrm{r}, \theta)$ space. In this polar coordinate space, track-like sets of hits will be pronounced as peaks. By identifying these peaks, one can identify tracks. 


\subsubsection{Track Fitting}

After a track is found, it is fitted using a Kalman filter. A Kalman filter is a recursive filter that estimates the state of a dynamic system from a series of incomplete and noisy measurements. The result of this step is to provide the charge sign of the track and the momentum from curvature using the magnetic field that both Near and Far detectors have.

\subsubsection{Event Formation}

In this final step, one takes the full list of showers and tracks and combines them to form events. The spatial separation and topological criteria are used to find showers and tracks that are compatible. This formation stage is the best estimate of the energy depositions caused by a neutrino interaction. charged current $\nu_{\mu}$ events will typically have a reconstructed $\mu$ track and a shower that corresponds to its hadronic activity. A neutral current event will typically contain only a shower or a shower with a track deeply imbedded inside of it.

\subsection{Summary}

We have outlined the main aspects of the reconstruction software. Individual signals in the detector are first formed into "digits" and then further processed into "strips" which are the basic units of our energy signal. These strips are then processed with the slicing algorithm to further separate localized interactions within the Near detector only. These slices are then passed onto track and shower finding algorithms to locate particle tracks and hadronic or electromagnetic showers in the detector. Finally, we form neutrino events by comparing the shower and track lists.

In the next chapter we use this information to properly select charged current and neutral current events to be used our measurement of $f_{\text {sterile }}$. 


\section{Chapter 5}

\section{Event Classification in the Near}

\section{Detector}

We discuss here the selection algorithm used to categorize charged current and neutral current events in the MINOS Near detector. This sample of events will be used to resolve differences between our data and Monte Carlo simulation. The plots in this chapter are produced with the nominal beam configuration where the target position is $10 \mathrm{~cm}$ and the horn current is set at $-185 \mathrm{kA}$ unless otherwise stated. Far detector events are selected in a similar fashion but have some extra requirements that we will discuss in Chapter 7. All comparisons between data and Monte Carlo are normalized by the total protons on target used in the sample.

The chapter begins by discussing how to select quality beam spills by looking at information from the monitoring devices along the NuMI beamline. After proper beam spills are selected, fiducial volume containment criteria are applied to all events ensuring that they are properly contained in the detector so their energy does not leak out. A brief overview is given on a set of criteria used to remove low completeness events that were described in the previous chapter. We then move onto our method for selecting charged current events. Selecting charged current 
events is discussed first because ultimately we use them for tuning our neutrino flux simulation to correct for hadron production systematic errors on our target. After defining the charged current selection, a discussion of studies undertaken to tune our neutrino flux model follows. These corrections to the flux will be applied to all Monte Carlo. Last, neutral current event selection is discussed and we finish with a final selected charged current and neutral current energy spectrum in the Near detector and a summary of the application of all our selection criteria.

\subsection{Selecting Quality Beam}

Before we can select neutrino events in the Near detector we must ensure that the beam spills were recorded with stable and "good beam" conditions [96]. There are a variety of monitoring devices taking data on the NuMI beam line. We use Secondary Emission Monitors to measure the profile of the beam and Beam Position Monitors to measure the position with respect to the NuMI target [97].

The initial beam cuts applied to data were as follows:

- $0.1 \mathrm{~mm}<\sigma_{h}<1.5 \mathrm{~mm}$

- $0.1 \mathrm{~mm}<\sigma_{v}<1.7 \mathrm{~mm}$

- protons per pulse $>0.5 \times 10^{12}$ and $<50 \times 10^{12}$

- $-190 \mathrm{kA}<$ Horn Current $<-160 \mathrm{kA}$

- $-2.0 \mathrm{~mm}<$ Horizontal Position $<0.0 \mathrm{~mm}$

- $0.0 \mathrm{~mm}<$ Vertical Position $<2.0 \mathrm{~mm}$

- $\operatorname{abs}[($ data - base time $)-$ snarl time $)]<1$ second

where $\sigma_{h}$ and $\sigma_{v}$ are the horizontal and vertical width of the beam. The horizontal and vertical positions are defined with respect to the target. 

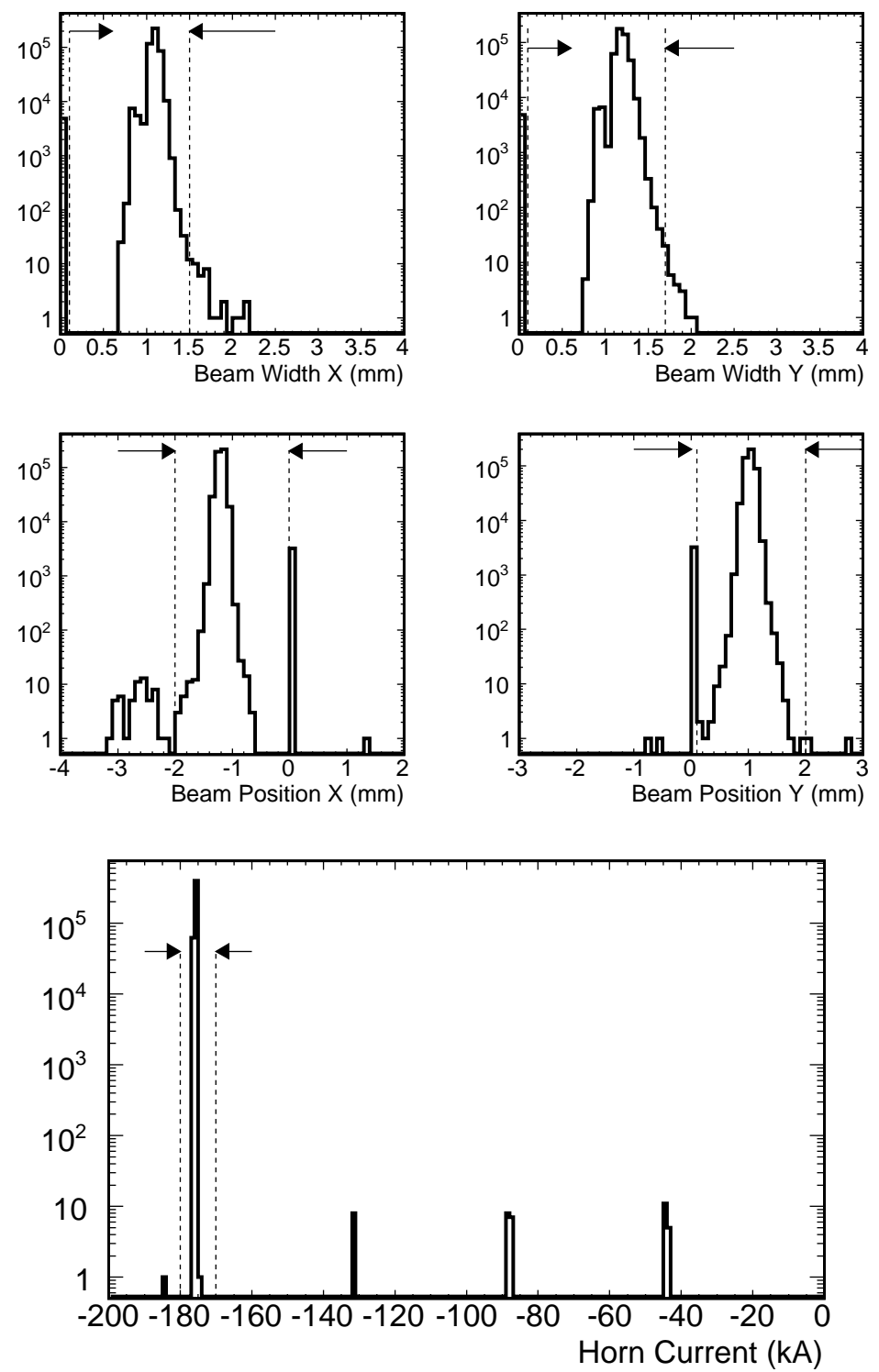

Figure 5.1: The top two plots are the horizontal, vertical beam positions and with respect to the target coordinate system. The middle plots show the horizontal and vertical beam widths and at the bottom we show the measured magnetic horn current. The dashed lines and arrows indicate what sample of beam is used. Note that the $y$-axis is on a log scale. 
The magnetic horn current has to be within the possible ranges used to produce different beam configurations. The protons per pulse can not go below $0.5 \times 10^{12}$ which are mostly test spills, and cannot be above $50 \times 10^{12}$ which is above the designed capacity of $40 \times 10^{12}$.

Last the difference between the raw spill time from the data files and the time from the beam data base cannot be more than 1 second. This is because the two times are written out separately and some clock jitter can occur, but anything more than a second difference is suspect. In Figure 5.1 we plot the beam widths, position, and horn current for a sample of beam spills. After applying these criteria, one can be assured of quality neutrino beam spills.

\subsection{Fiducial Volume Containment}

To have an accurate measurement of the energy of neutrino interactions, one has to ensure that the energy from these interactions is wholly deposited inside the detector. This is more of an issue for hadronic showers than for long $\mu$ tracks, since one can reconstruct their momentum using the magnetic field. A spatial set of fiducial volume cuts must be put in place to properly contain events. We define our fiducial volume to be from $1.728<\mathrm{Z}<4.7368 \mathrm{~m}$ longitudinally so that the neutrino interaction occurred well within the calorimeter section of the Near detector and far enough away from the start of the spectrometer so that hadronic showers are fully contained longitudinally. Also, the transverse vertex cannot be more than $50 \mathrm{~cm}$ from the edge of the partial scintillator planes. This is so no energy leaks out the edges. We show the result of these cuts in Figure 5.2. 


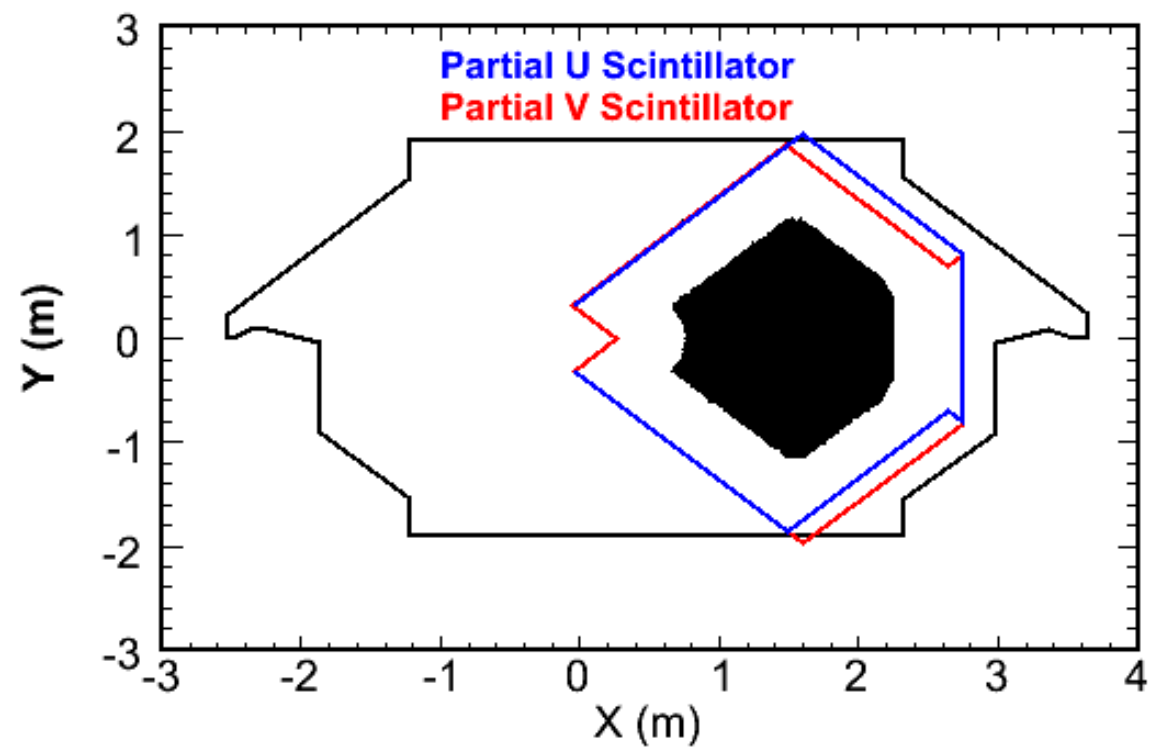

Figure 5.2: The front face of the Near detector steel plane outline. The blue outline is the partial U scintillator plane and the red is the corresponding partial V scintillator plane. The black dots are the vertices of events that were selected as being fiducially contained.

\subsection{Data Cleaning}

There are several background sources that "pollute" the first two energy bins (0$1 \mathrm{GeV}$ ) of the neutral current visible energy spectrum. They can generally be categorized into reconstruction failures from slicing, vertex failures where an event interacts outside the detector and a small bit of energy enters, and some more subtle effects such as the shower reconstruction making a separate shower out of a cluster. Also a $\mu$ track that produces a $\delta$-ray can be picked up by the shower reconstruction as a separate shower. The event reconstruction in that case fails to associate the track and the $\delta$-ray shower together. All of these errors can be categorized as low completeness events, defined in the event isolation section in Chapter 4 . We are primarily concerned with events with a completeness $<50 \%$. Since only a small fraction of the true total energy is recorded, the energy of these low completeness 
events will be systematically too low and presents a bias in our analysis. It is necessary to remove these types of events.

A set of cleaning requirements [98] have been developed. The cleaning requirements are a combination of timing and topological cuts. If two reconstructed events are caused by the same neutrino interaction, they should be close in time and position, hence using the minimum time separation $\Delta t$ and the minimum difference in $\mathrm{Z}$ vertex $\Delta z$ between two events will help to eliminate these classes of events. We show the distributions of these two quantities in Figure 5.3. The blue portion of the spectrum is the low completeness portion and one can see that it is heavily populated in regions of close time and spatial separation. In addition to these two important variables, certain topological cuts are performed to eliminate low completeness events that are caused from something leaking into the Near detector.

Very steep showers cause problems for the vertex of shower algorithms or because events that interacted outside of the detector leaked into the sparsely instrumented regions at the side of the Near detector. One can define a "steepness" variables which is defined as the total number of strips in the shower divided by the longitudinal length in planes squared.

$$
\text { Steepness }=\frac{\text { Number of strips in the shower }}{\text { Length of the Shower (Number of Planes) }}
$$

A high value for steepness means that the shower spans very few planes but has many strips. It is likely that the event is due to activity entering the detector from the side. Other variables are defined in terms of what percentage of the hits in the event are on the outer edges of the detector. These cuts also help to eliminate low completeness events. The final product of these cleaning cuts is shown in the event completeness spectrum from Monte Carlo truth in Figure 5.4. These cuts take the low completeness fraction from $3.6 \%$ to $0.6 \%$. 

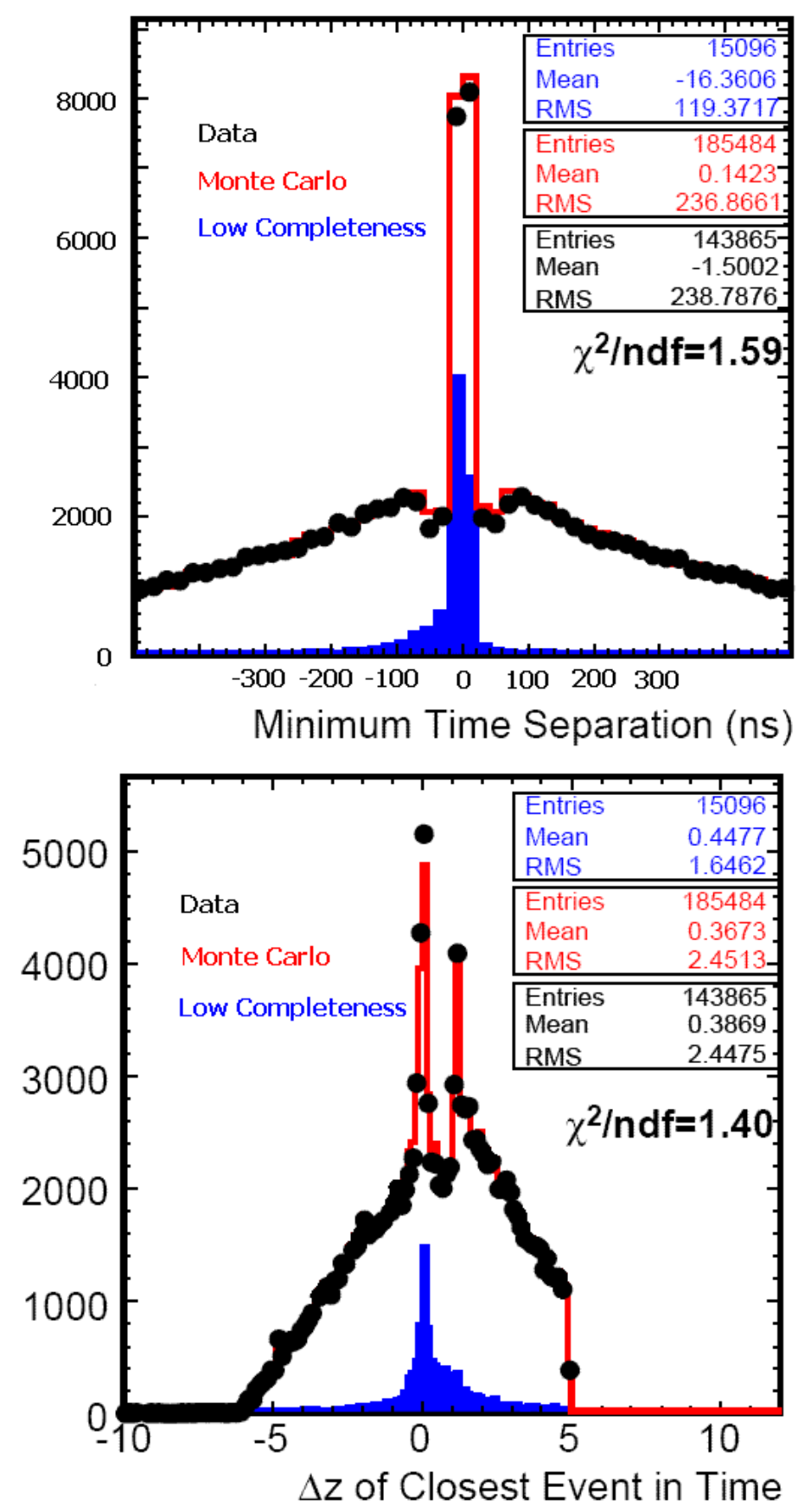

Figure 5.3: The top plot is a distribution of the minimum separation between two events in time. The bottom plot is a spatial distribution of the minimum distance in $\mathrm{Z}$ between two events. The black is data, the red is the Monte Carlo and the blue portion is the low completeness fraction of the Monte Carlo. 


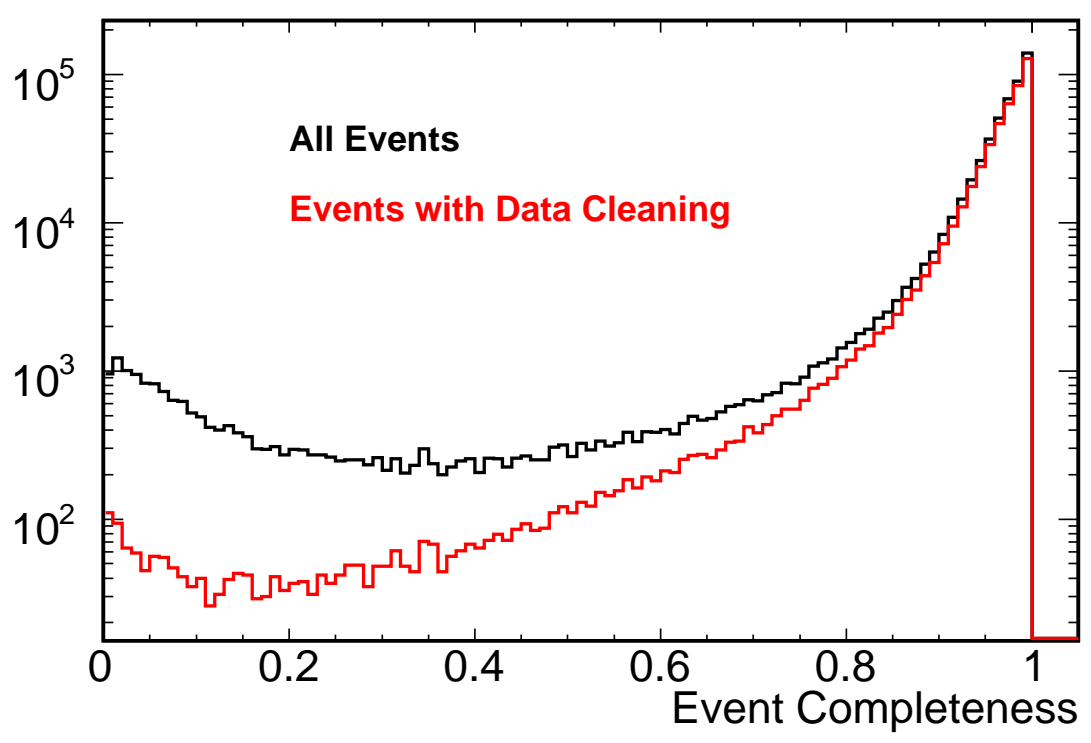

Figure 5.4: Completeness of events shown before the data cleaning cuts in black, and with the cleaning cuts in red.

\subsection{Charged Current Event Selection}

Charged current events are selected using a likeli-hood based particle identification parameter (PID). The PID is based on three event probability density functions (PDFs). The first PDF is the event length in units of number of planes. Charged current events produce a muon track which penetrates deep into either detector giving the event a long length, while neutral current events are much shorter. The second PDF is the average track pulse height per plane. If the event in question has a track, we define this variable as the total pulse height of the track in ADC counts divided by the total length of the track. Again, since charged current events produce muon tracks, this variable is expected to be peaked around the average $\frac{d E}{d x}$ of the muon, while tracks found in neutral current events will cause this variable to be broader. The third PDF is defined as the fraction of energy in an event that is contained in a reconstructed track. If an event did not have a track, this would be 
zero. By capitalizing on the existence of muon tracks in charged current events we expected this variable to have higher values than for a neutral current event, since they are more shower-like rather than track-like.

The probability that a particular event is consistent with the $\nu_{\mu}$ charged current or neutral current PDFs is given by the product of the three individual probabilities. The separate probabilities for charged current and neutral current are given by

$$
\begin{aligned}
& P_{C C}=\prod_{i=1}^{3} P D F_{C C i} \\
& P_{N C}=\prod_{i=1}^{3} P D F_{N C i}
\end{aligned}
$$

The charged current PID is then defined as

$$
P I D=\sqrt{-\log P_{N C}}-\sqrt{-\log P_{C C}}
$$

Events that are more likely to originate from $\nu_{\mu}$ charged current interactions are assigned positive values, and those that are more likely to be neutral current are assigned negative values.

Figure 5.5 shows the three PDFs and the resulting PID distribution for Near detector data and Monte Carlo. For the Near Detector a PID value $>-0.1$ is applied, while a value of $>-0.2$ for the Far is used. These values were chosen to optimize the sensitivity to oscillations [100].

\subsection{Neutrino Flux Tuning}

Incomplete knowledge of hadron production in the NuMI target gives a uncertainty on the neutrino flux. MINOS has undertaken measurements with Near detector charged current energy spectra to tune the Monte Carlo prediction [101]. The 

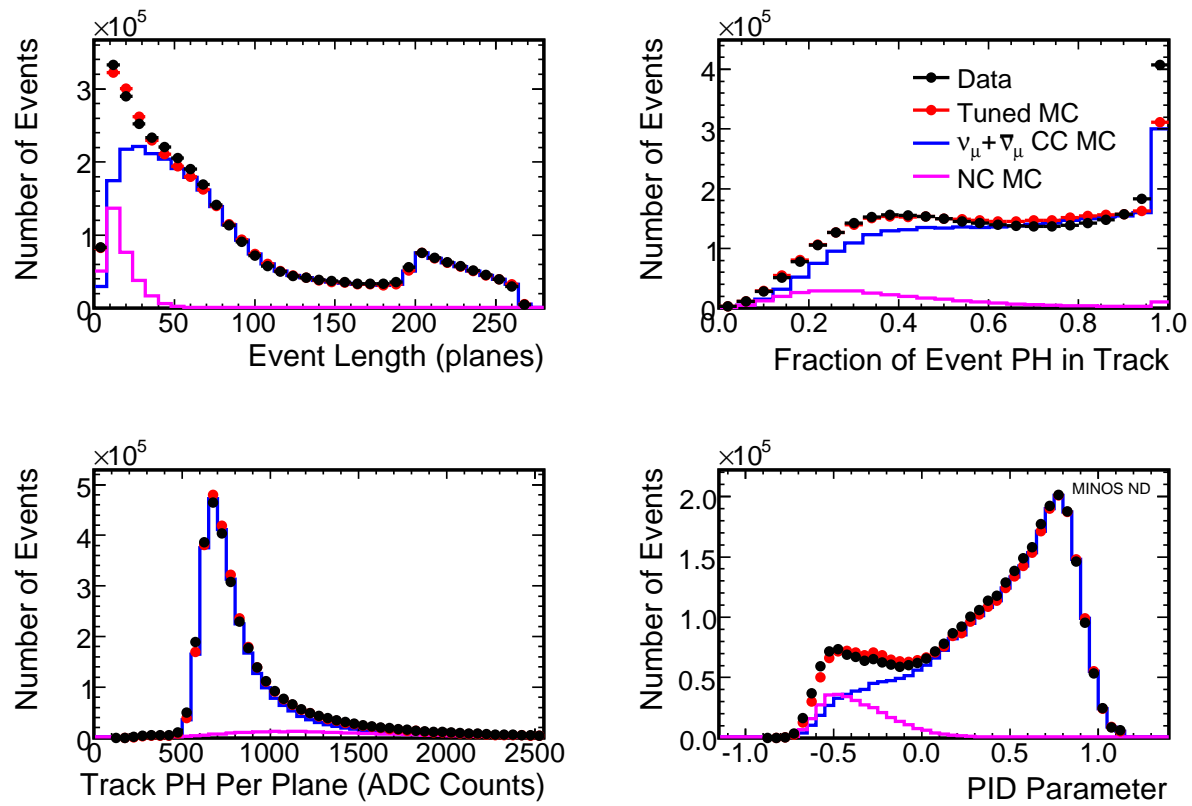

Figure 5.5: Data and tuned Monte Carlo predictions for event length, track/event pulse height fraction and average pulse height per track plane, as well as the PID variable derived from these quantities in the Near detector. The Monte Carlo neutral current and muon neutrino and anti-muon neutrino charged current breakdowns are shown. Error bars on MC distributions are statistical only. 
results of this study are individual weights given to the Monte Carlo to correct for this uncertainty. All plots following this section will include Monte Carlo with these weights.

Little experimental data of hadron production on specific materials exists. Specifically for MINOS' case, hadron production on a thick carbon target with a $120 \mathrm{GeV}$ proton beam simply does not exist in any useful form.

The uncertainty on the neutrino flux can be represented by the hadron yield $\mathrm{d}^{2} \mathrm{~N} / \mathrm{d} x_{F} \mathrm{~d} p_{T}$ as a function of the transverse momentum $p_{T}$ and the Feynman scaling variable $x_{F}$ defined as

$$
x_{F}=\frac{p_{L}}{p_{L, \max }}=\frac{2 p_{L}}{\sqrt{s}}
$$

where $p_{L}$ is the longitudinal momentum of the hadron and $\sqrt{s}$ is the center of mass energy of the interaction. These two quantities matter for this study because the magnetic horns are limited in $p_{T}$ and there exists a relationship between $x_{F}$ and neutrino energy.

Only charged current events are used because they measure the total neutrino energy, while neutral current events are only the partial energy and have little sensitivity to these parameters. Charged current events are selected as described in the previous section.

The basic idea of the study is to use an empirical function $f\left(x_{F}, p_{T}\right)$ to model $\mathrm{d}^{2} \mathrm{~N} / \mathrm{d} x_{F} \mathrm{~d} p_{T}$. In addition to $x_{F}$ and $p_{T}$ additional parameters were added to account for beam focusing and detector modeling errors which include a energy miscalibration, shower miscalibration, and a scaling on the neutral current background. These extra parameters were added in order for the fits performed to stay in physical regions of $x_{F}$ and $p_{T}$. One then uses $f\left(x_{F}, p_{T}\right)$ to reweight the Monte Carlo using a fit to the Near detector data. 

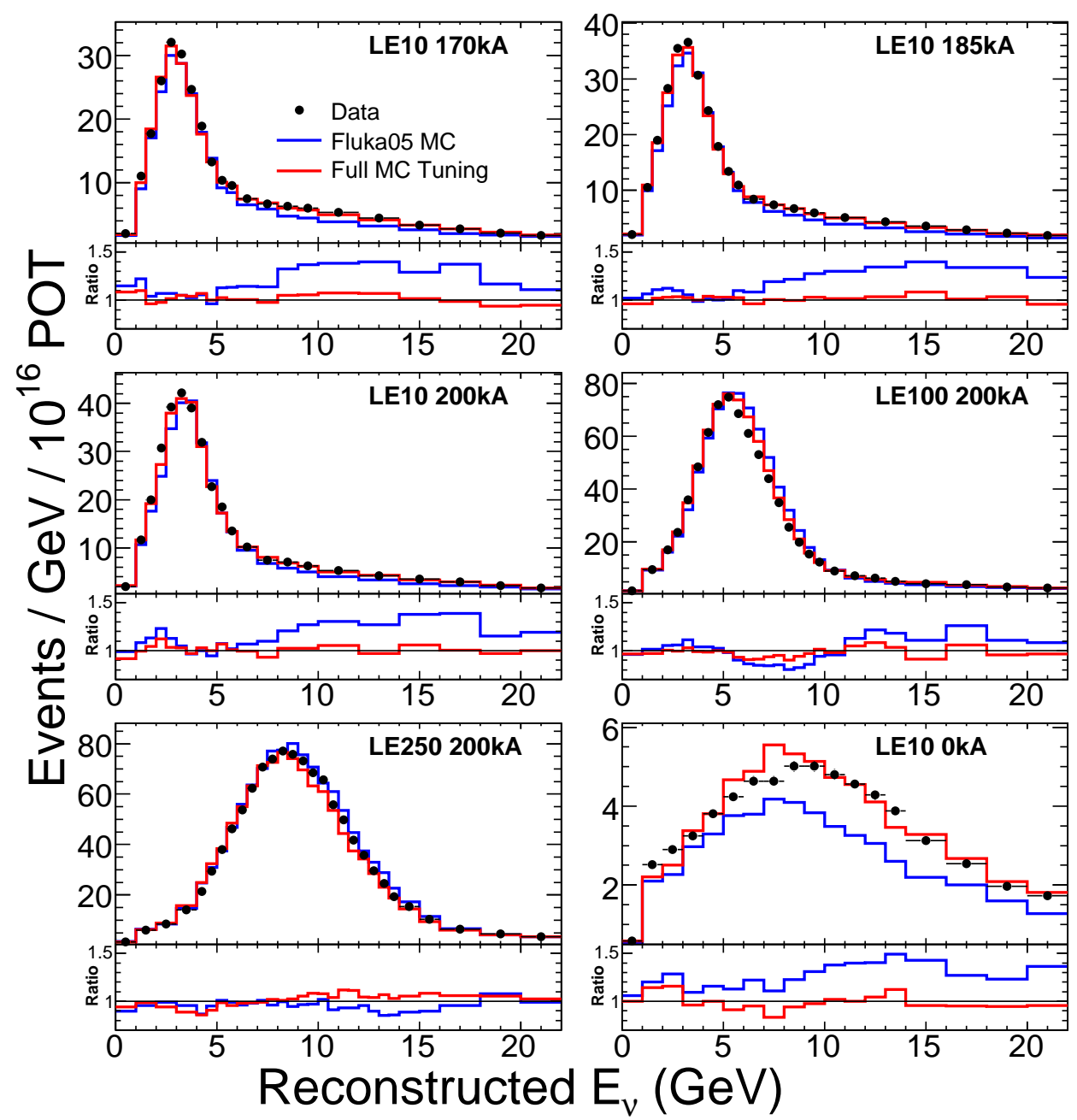

Figure 5.6: Energy spectra in the MINOS ND for six beam configurations before and after the beam tuning procedure. The NuMI target and horn current were modified to produce the different spectra: a) Target position at $10 \mathrm{~cm}$, horn current at $170 \mathrm{kA} \mathrm{b}$ ) Target position at $10 \mathrm{~cm}$, horn current at $185 \mathrm{kA} \mathrm{c}$ ) Target position at $10 \mathrm{~cm}$, horn current at $200 \mathrm{kA} \mathrm{d}$ ) Target position at $100 \mathrm{~cm}$, horn current at 200 $\mathrm{kA}$ e) Target position at $250 \mathrm{~cm}$, horn current at $200 \mathrm{kA} \mathrm{f}$ ) Target position at 10 $\mathrm{cm}$, horn current at $0 \mathrm{kA}$ The lower inset shows the ratio of data to $\mathrm{MC}$ before and after tuning. The error bars on the data points are smaller than the points. 
Table 5.1: Different beam configurations used for neutrino flux studies

\begin{tabular}{|c|c|c|c|}
\hline $\begin{array}{c}\text { Beam } \\
\text { Configuration }\end{array}$ & $\begin{array}{c}\text { Target } \\
\text { Position }(\mathrm{cm})\end{array}$ & $\begin{array}{c}\text { Magnetic Horn } \\
\text { Current }(\mathrm{kA})\end{array}$ & $\begin{array}{c}\text { Total Protons } \\
\text { On Target }\left(\times 10^{18}\right)\end{array}$ \\
\hline \hline L010z170i & 10 & -170 & 1.34 \\
\hline L010z185i & 10 & -185 & 2.85 \\
\hline L010z200i & 10 & -200 & 1.26 \\
\hline L100z200i & 100 & -200 & 1.10 \\
\hline L250z200i & 250 & -200 & 1.55 \\
\hline L010z000i & 10 & 0 & 2.69 \\
\hline
\end{tabular}

Six different beam configurations are used for this study. As described in Chapter 3 , by varying the target position and horn current, one can produce different energy spectra in the Near detector. The nominal configuration that ultimately produce our oscillation results is having the target position at $10 \mathrm{~cm}$ and the horn current at $-185 \mathrm{kA}$. The different energy spectra and corresponding total protons on target for each sample used in this study is given in Table 5.1. The results of the fit are shown in Figure 5.6.

In Chapter 8, where systematic studies are detailed, the results of this flux tuning are changed within allowed errors to see the effects of the uncertainty in hadron production on our oscillation measurements.

\subsection{Neutral Current Selection Variables}

The goal of this event selection is to maximize the efficiency and purity of a selected sample of neutral current events. Charged current and low completeness events are the two main sources of background. These selection variables remove charged current events, while only data cleaning as defined previously can remove low completeness events efficiently. Figure 5.7 shows agreement between data and Monte Carlo for the neutral current selection variables. The variables are as follows: 

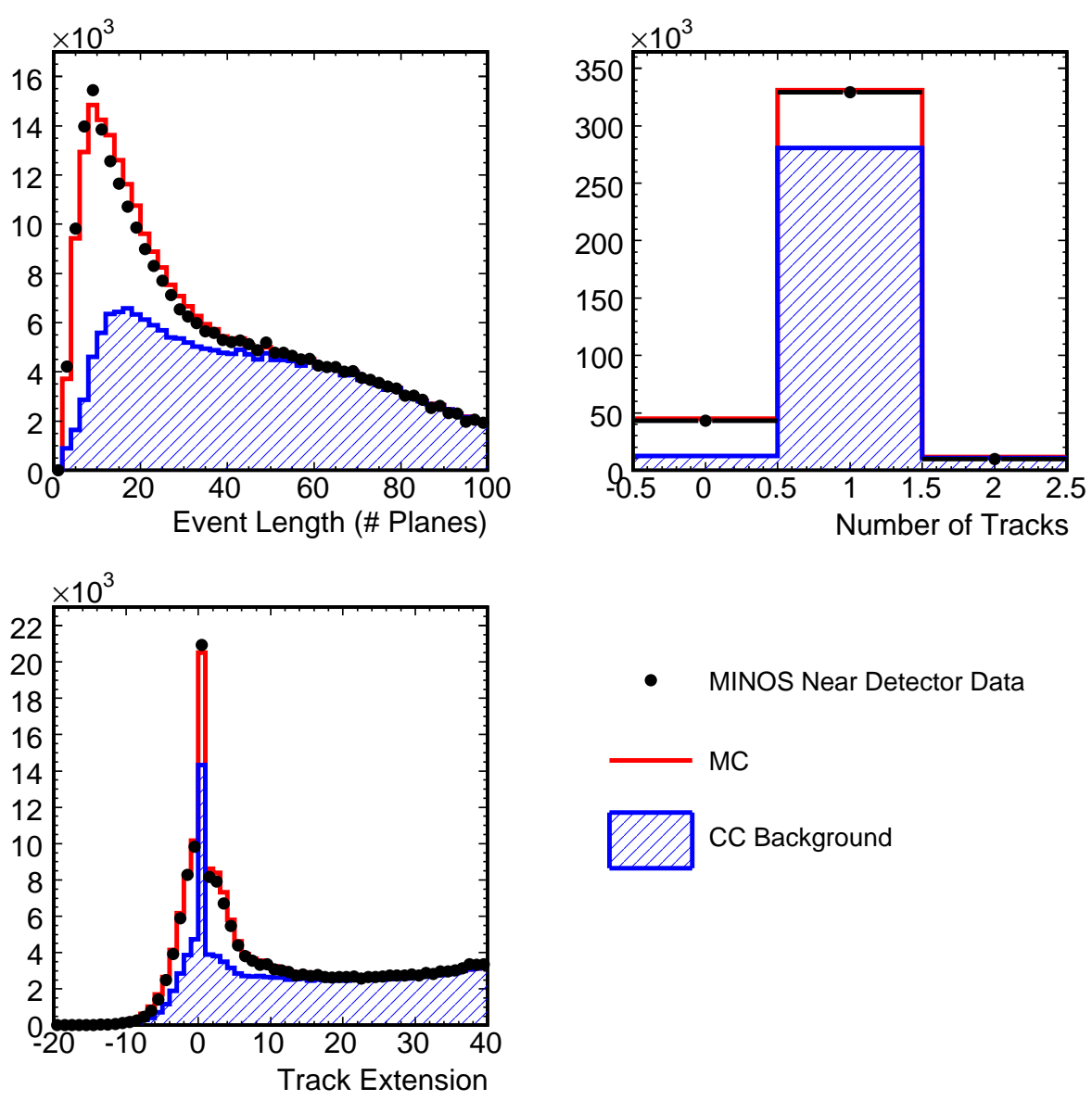

Figure 5.7: Selection Variables used for a Cut based Neutral Current event Selection.

- Event Length

- Number of Tracks Reconstructed

- Difference in track and shower lengths (Track Extension)

We select neutral current like (NC-like) events using the following criteria. Events spanning at least 60 planes are classified as charged current like (CC-like) and excluded immediately. The remaining events which have only a reconstructed shower are classified as NC-like. Those events which have both a reconstructed 
shower and track are classified as NC-like if the track is no more than 5 planes longer than the shower. The cuts applied to the selection variables are as follows:

- Event Length of less than 60 Planes

- If Event has no track classify it as NC-like

- If the Event has a track, then classify it as NC-like if the track is no more than 5 planes longer than the shower.

The first cut on event length is extremely efficient at removing charged current events. Virtually no neutral current events are lost. After this cut, one examines whether the remaining events have a reconstructed track. If they do not, then consider the event a NC-like event. True charged current and neutral current events in this category are almost indistinguishable. For events that have a track, we have an important piece of information, which is the difference in shower and track lengths. A true charged current event has a higher probability of a track that extends out of the main shower. A suitable cut at 5 eliminates an almost entirely charged current sample. The same cuts are applied to select NC-like events in the Far detector.

The particular cuts of event length $<60$ planes and track extension $<5$ planes were chosen because they maximize sensitivity to $f_{\text {sterile. }}$. The procedure was to select neutral current events allowing the event length cut to vary between $\{25,30,35,40,45,50,55,60,65\}$ and allowing the track extension cut to vary between $\{0,5,10,15,20,25,30\}$. This produces a number of different neutral current selections each having a different efficiency and purity as shown in Figure 5.8.

We define efficiency as the number of events selected as NC or CC-like divided by the true total number of neutral current or charged current events predicted from the Monte Carlo. The purity is the number of true charged current or neutral current events in a given energy bin divided by the total number of events in that bin. 

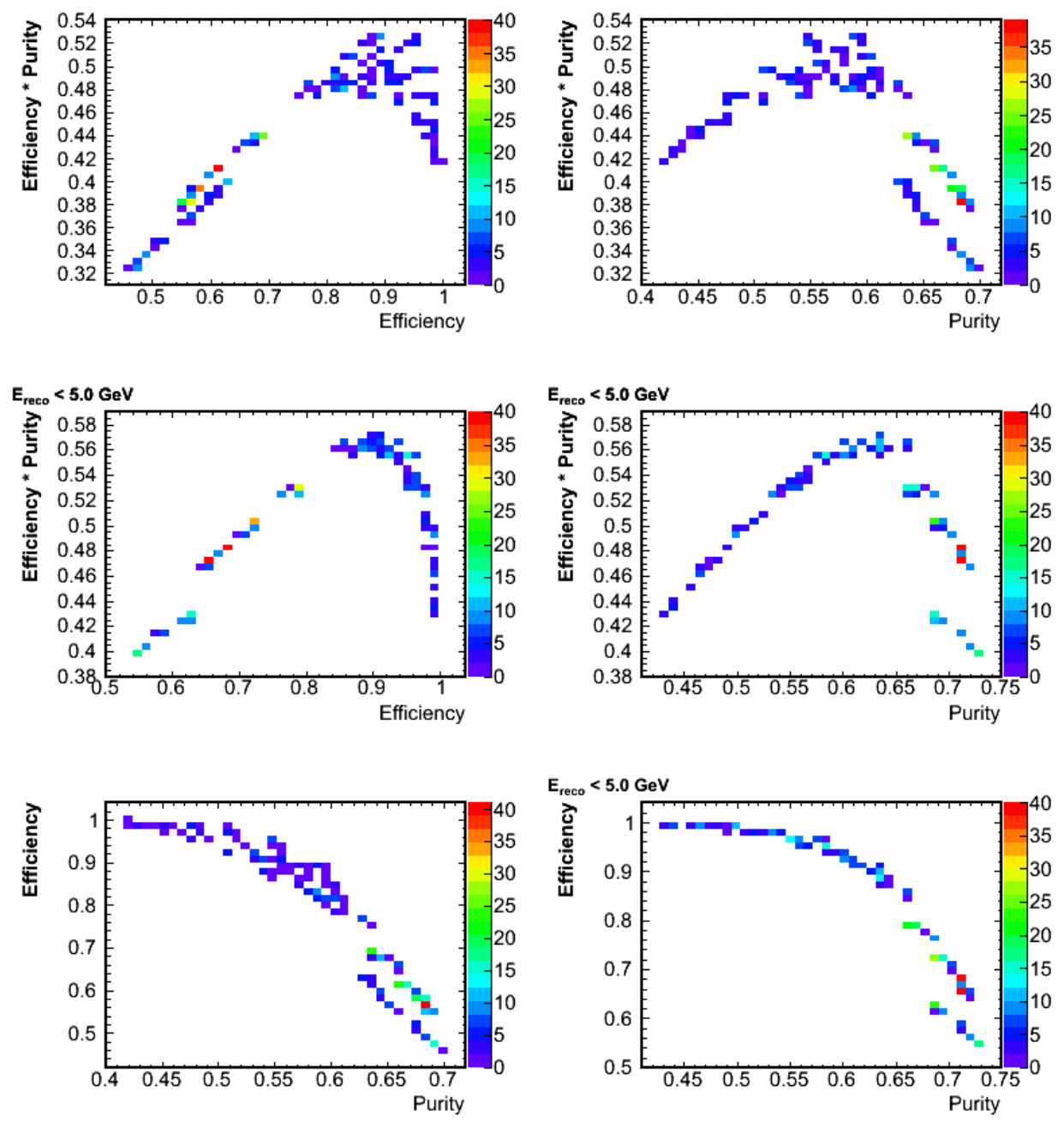

Figure 5.8: Efficiency, purity and the product of efficiency and purity vs efficiency and purity for all combinations of the Neutral Current selection.

Deviations from a purity of 1 imply that the energy bin is contaminated with either true neutral current events, in the CC-like case, or true charged current events in the NC-like case.

We used these various spectra as a "fake" data set in which we oscillated the sample using an $f_{\text {sterile }}=0.2$, and used it again as our Monte Carlo to fit for 
$f_{\text {sterile. }}$ Since this study uses a very high statistics Monte Carlo sample of $1.04 \times 10^{22}$ Protons on Target, each fit recovered the correct value of $f_{\text {sterile }}=0.2$. There were differences in the $69 \%$ Confidence Level upper bound. It was found that maximizing the product of efficiency and purity would yield the best sensitivity as illustrated by Figure 5.9, which shows the product of efficiency and purity on the x-axis and the $69 \%$ confidence level upperbound on our fit for $f_{\text {sterile }}$. We see that maximizing this product gives the lowest error on $f_{\text {sterile }}$.

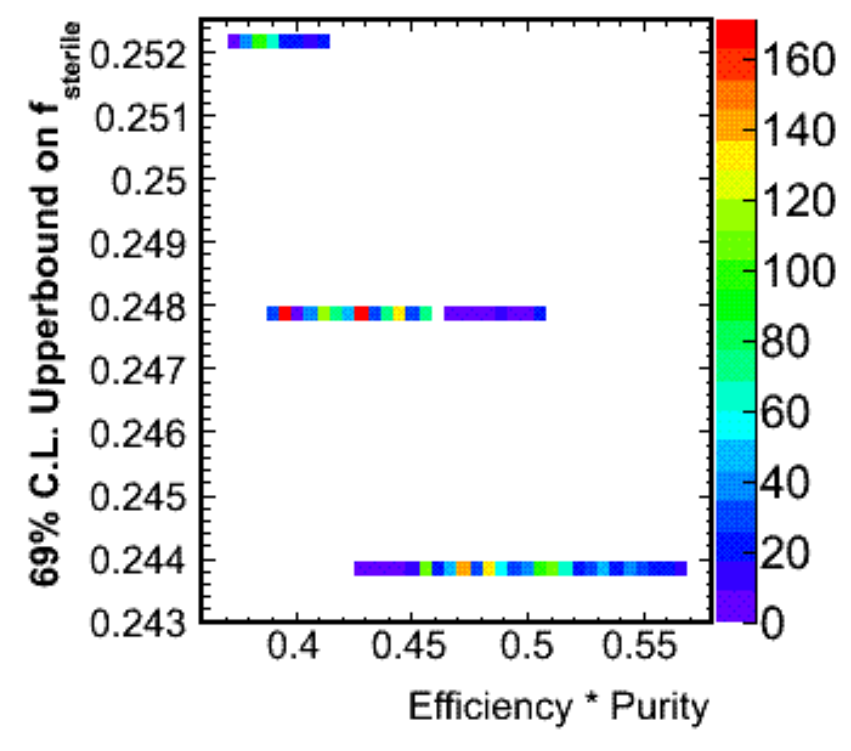

Figure 5.9: A summary of all the $69 \%$ C.L. upper bounds on a fit to $f_{\text {sterile }}$ versus the product of efficiency and purity. Clearly the error is smallest when this quantity is maximized. 


\subsection{Near Detector Charged Current and Neutral Cur- rent Energy Spectra}

In this section we show the results of the CC-like and NC-like selections used for this analysis. We show plots using the nominal beam configuration with the target position at $10 \mathrm{~cm}$ and horn current at $-185 \mathrm{kA}$ (L010z185i). Every event is first passed through the neutral current selection. If it passes this selection is it automatically labeled NC-like, otherwise it is passed through the charged current selection. If it passes that it is labeled CC-like, and if it fails, then the event is not used in the analysis.

The energy definitions of both NC-like and CC-like events are defined in terms of the energies of tracks and showers associated with the event. In certain cases, more than one shower or track can be associated with each event. In these cases we must define the primary shower and primary track in order to define and total energy for the event.

If an event has more than one shower reconstructed, the first criteria to be considered the primary shower is that the shower vertex be no more than one meter away from the event vertex. If this is satisfied, the shower with the most energy is considered the primary shower. The primary track is defined as the track with the most energy in the event.

We define the energy of charged current events as the energy of the primary shower plus the energy of the primary track given by

Charged Current Energy $(\mathrm{GeV})=$ Shower Energy $(\mathrm{GeV})+$ Track Energy $(\mathrm{GeV})$

Neutral current events physically consist of only a hadronic shower, hence we define 
the energy of neutral current events to be the energy of the primary shower:

Neutral Current Visible Energy $(\mathrm{GeV})=$ Shower Energy $(\mathrm{GeV})$.

The neutral current and charged current energy spectrum is shown in Figure 5.10. We show both the nominal Monte Carlo and the reweighted Monte Carlo with corrections from the neutrino flux tuning superimposed. Figure 5.12 show the efficiencies and purities of the NC-like and CC-like energy spectrum as a function of visible energy and true energy from the Monte Carlo.

If we examine both energy spectra in Figure 5.10 we see that we model the neutrino interactions reasonably well in our detector. The largest deviations between data and Monte Carlo exist at lower energies for both the charged current and neutral current spectra. Higher energies are typically better modeled and lie very close to the corrected Monte Carlo given by the red solid line in each spectra. In order to have a prediction for the Far detector unoscillated energy spectrum, our procedure will fit the Near detector for a set of systematic parameters in order to get agreement between data and Monte Carlo. The results of this fit will then be applied to the Far detector to gain a prediction. These issues will be discussed later in the analysis.

Notice also that the CC-like spectrum has a low background of neutral current events, while the NC-like spectrum has a higher background of charged current events. Neutral current events are easier to eliminate since CC-like events generally have well reconstructed $\mu$ tracks. The charged current background is higher in the NC-like spectrum because there do exist a class of true charged current events that have very short, poorly reconstructed tracks, and it is extremely difficult to distinguish between the two. In some cases, true neutral current events have a reconstructed track that comes from a charged pion or proton as well. Much effort has gone into eliminating the charged current background in the NC-like spectrum. 
Table 5.2: The effect of selection criteria on data and Monte Carlo

\begin{tabular}{|c|c|c|c|c|}
\hline Cut & $\begin{array}{c}\text { \# of Data } \\
\text { Events }\end{array}$ & $\begin{array}{c}\text { \% of Data } \\
\text { Events }\end{array}$ & $\begin{array}{c}\text { \# of MC } \\
\text { Events }\end{array}$ & $\begin{array}{c}\text { \% of MC } \\
\text { Events }\end{array}$ \\
\hline \hline None & 6350751 & 100 & 8372472 & 100 \\
\hline Fiducial Volume & 435119 & 6.85 & 583088 & 6.96 \\
\hline Data Cleaning & 382397 & 6.02 & 508500 & 6.07 \\
\hline
\end{tabular}

Table 5.3: The effect of selection criteria on low completeness events in the Monte Carlo

\begin{tabular}{|c|c|c|}
\hline Cut & \# of Low Completeness Events & \% of Low Completeness Events \\
\hline \hline None & 467180 & 100 \\
\hline Fiducial Volume & 21340 & 0.25 \\
\hline Data Cleaning & 3075 & 0.036 \\
\hline
\end{tabular}

Despite the numerous selection criteria developed by the Neutral Current Analysis Group, it seems unlikely that this background will be significantly reduced [102].

We document the percentage of events eliminated as a function of our selection criteria. This is shown in data and Monte Carlo in Table 5.2 and for low completeness events in the Monte Carlo in Table 5.3. Notice that the final percentages of selected events between data and Monte Carlo agree to within $0.05 \%$. 

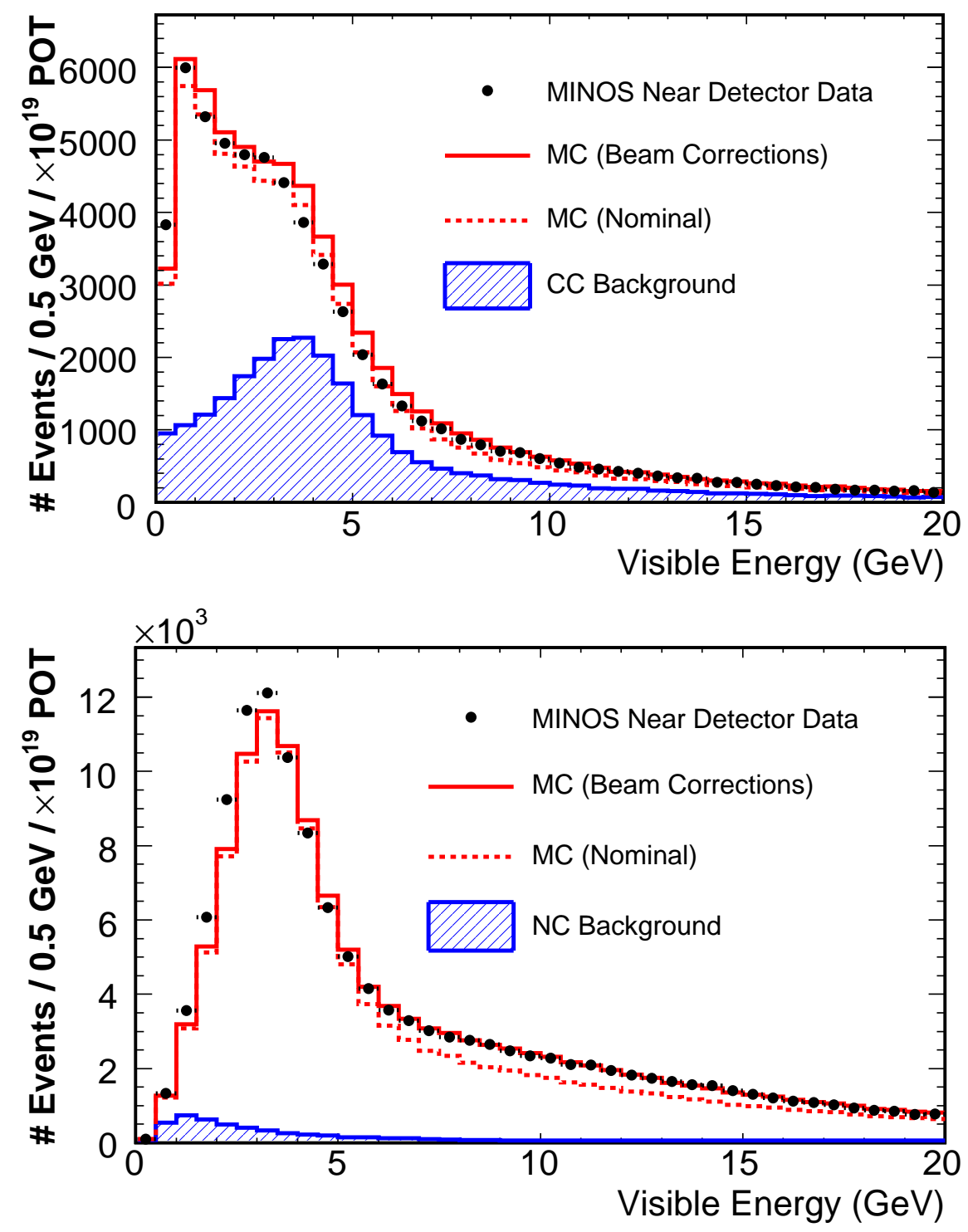

Figure 5.10: Neutral Current and Charged Current selected energy spectra. The top plot is NC and the bottom plot is CC. The black dots are data, the dashed red is the Monte Carlo without Neutrino Flux corrections and the red is with the corrections applied. The blue shaded portion of each spectrum is the corresponding $\mathrm{CC}(\mathrm{NC})$ Background in the NC (CC) spectrum. 

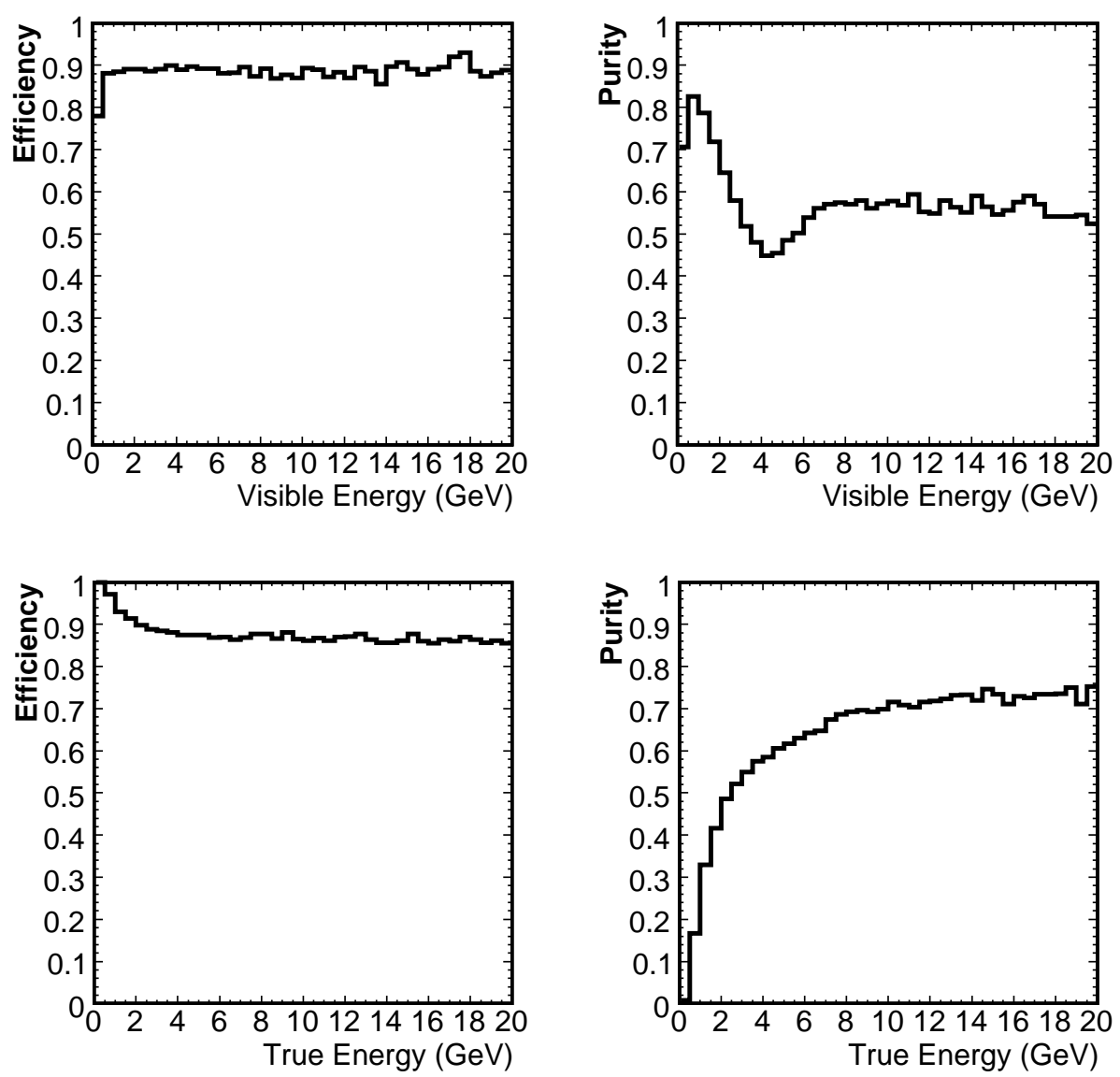

Figure 5.11: Efficiencies and Purities as a function of visible and true energy from Monte Carlo for the Neutral Current selection. 

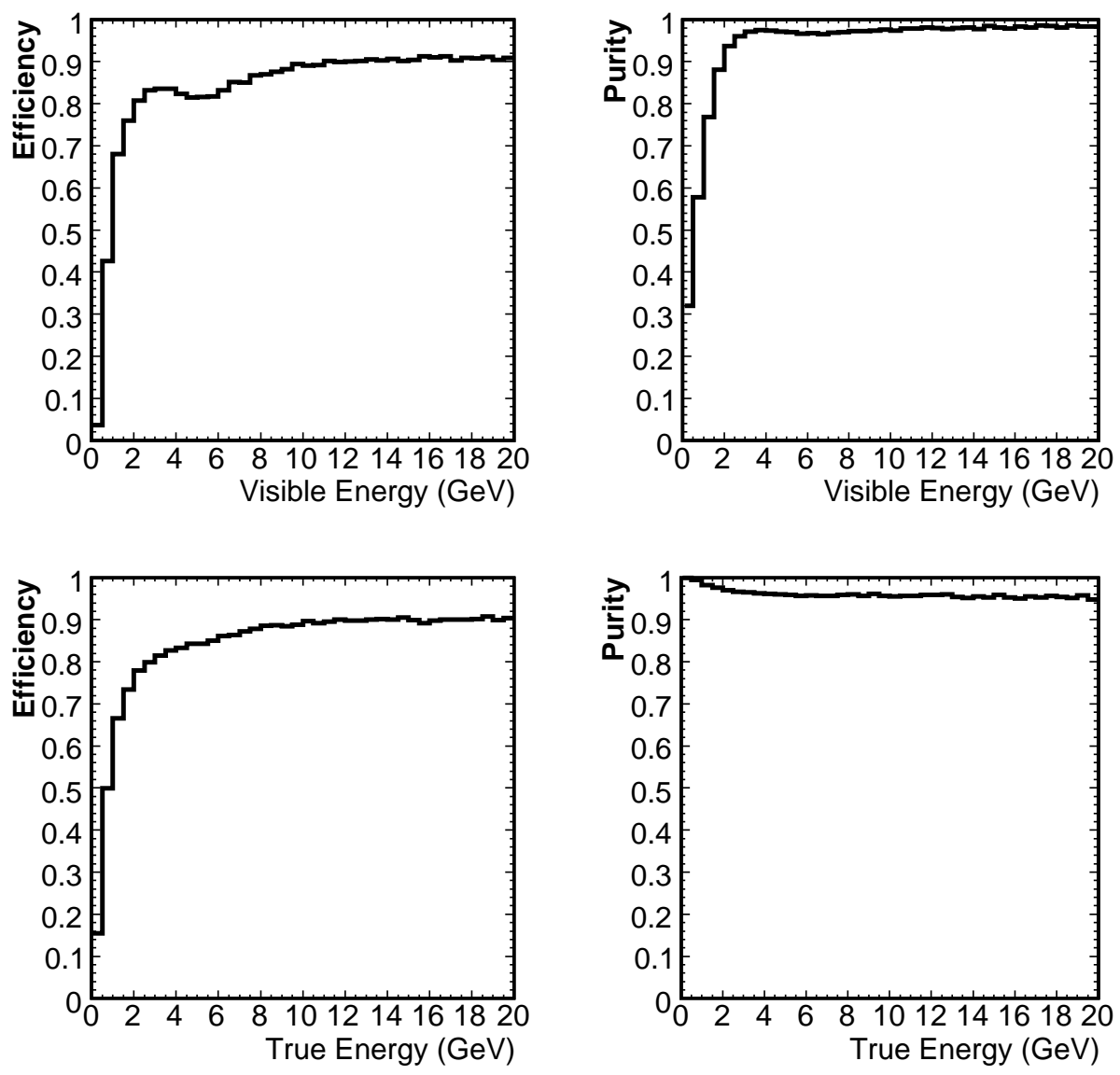

Figure 5.12: Efficiencies and Purities as a function of visible and true energy from Monte Carlo for the Charged Current selection. 


\subsection{Summary}

We have defined methods to select charged current and neutral current events in the Near detector. These same methods work exactly the same in the Far detector, except for additional criteria used to eliminate backgrounds which will be discussed. The outlined selections will be used throughout the rest of this work.

The next chapter outlines two studies undertaken to validate our understanding of the Near detector. The first deals with event whose vertices are in different parts of the detector. Any differences in reconstruction throughout the Near detector should be reproduced in Monte Carlo. Second, we undertake a study of beam intensity effects on our reconstruction. Again, we are looking to confirm that any physical changes in the detector due to intensity can be reproduced by Monte Carlo. 


\section{Chapter 6}

\section{Reconstruction Validation}

\section{Studies}

\subsection{Introduction}

Prior to making an oscillation measurement we must prove to ourselves that we understand our detectors. In particular, a simultaneous validatation of the reconstruction software and Monte Carlo simulation can be performed by looking for biases. Clearly, before the oscillation analysis is complete, it must be shown that the data is biased in an expected way, meaning that it is reproduced by our Monte Carlo simulation.

In this chapter the results of two studies are shown that test how well we model the reality of the Near detector and beam intensity issues. Due to the low amount of statistics in the Far detector, we focus on a Near detector sample of data of the nominal L010z185i. The first set of studies examines reconstructed energy spectra of events with vertices in different sections of the detector. This includes splitting the fiducial volume into longitudinal and transverse sections, in addition to different radial annuli. The second set of studies deals with the beam intensity 
issues brought up while discussing the Slicer algorithm. We present a set of variables normalized to each other by protons on target for three different beam intensities that we define later as low, medium, and high, to show that slight differences do exist between intensities. Next, we compare high intensity data to low intensity data, and similarly compare high intensity Monte Carlo to low intensity, and see if the same differences occur between intensities for data and Monte Carlo. Last we show results comparing different energy spectra from different NuMI batches. When protons are delivered to the NuMI beamline, they are received in a group of either five or six batches, which we discuss later. We wish to show that no differences exist between events from different proton batches. Each section has more detailed description of the plots.

\subsection{Detector Position Studies}

If one is properly modeling their detector then changes between energy spectra in different sections of the detector should be the same between data and Monte Carlo. To verify this, we plot the total neutrino energy, shower energy, and track energy for charged current events, and the total visible energy for neutral current events in different areas of the Near detector. We modify our original fiducial volume only slightly to compare sections of the Near detector with equal areas. The longitudinal requirement still requires event vertices be between $1.728<\mathrm{Z}<4.737$. Radially we require the event vertex be contained within a radius of $0.8 \mathrm{~m}$ around the beam center, defined to be at $\mathrm{x}=1.4885$ and $\mathrm{y}=0.1397$. This cylindrical fiducial volume allows us to easily segment the detector into equal areas.

The first set of Figures 6.1 through 6.4 shows previously listed quantities for events whose vertex is in one of four transverse quadrants, segmented from the cylindrical fiducial volume.

Let us take Figure 6.1 as an example of the plot format. The top left plot has 
four charged current total energy spectra, where each color corresponds to a different transverse quadrant. The legend shows a circle that is centered around the beam spot with a radius of $0.8 \mathrm{~m}$ and then segmented into four quadrants. The legend is positioned from the view of someone looking upstream at the Near detector. The top left shows this for data, while the top right plots the same quantities with Monte Carlo. The following middle two plots are the ratios of each of the four spectra to the total spectra. These ratios are centered at 0.25 , which one expects if the energy spectra are distributed evenly between the four quadrants. The left again is data and the right is Monte Carlo. Notice immediately that by eye one can see the data and Monte Carlo follow each other rather well. To illustrate this further, in the bottom plot we take the ratio of the corresponding color in each of the data and Monte Carlo in the middle two plots and plot the ratio of ratios. This ratio should be one if data follows Monte Carlo exactly. We also superimpose on the bottom the $\chi^{2}$ per number of degrees of freedom from the fit of the plotted ratio to the function $f(x)=1$. Notice that the $\chi^{2} /$ ndf is consistent with a good fit.

If one examines Figures 6.1 through 6.4, one notices that the spectra are very similar between quadrants. The main difference one notices, which happens to be modeled properly in Monte Carlo, is that there are less tracks reconstructed on the side of the fiducial volume where the magnetic coil exists. Tracks that curve towards the magnetic field coil have a tendency to enter the coil and exit the coil leaving a gap in the track which can cause reconstruction errors and hence an inefficiency in track finding. On the legend in the top left plot, this corresponds to the top left and bottom left quadrant, given by the green and blue colors.

Figures 6.5 through 6.8 are plotted in the same format, but instead of plotting quantities in different transverse quadrants, we plot them divided into three longitudinal segments. They are chosen such that each segment is the same longitudinal length. Since the longitudinal requirement for the fiducial volume is $1.728<$ 
$\mathrm{Z}<4.737$, we divide it into three parts where the division are at $\mathrm{Z}=[1.728,2.731$, $3.734,4.737]$. Examining all the $\chi^{2} /$ ndf on these plots draws the same conclusion as with the previous four.

Figures 6.9 through 6.12 show the fiducial volume divided into different radial annuli. There are three divisions that were chosen such that each annuli has the same area. We defined the cylindrical radius of the fiducial volume to be $\mathrm{R}<0.8 \mathrm{~m}$. If we divide this into three segments, we find the divisions at $R=[0.0,0.462,0.635$, 0.8]. Again, the figures show that data indeed follows Monte Carlo throughout the detector.

The final conclusions from the detector position studies is that the Near detector geometry is modeled very well since we see the same positional characteristics in our distributions. This also speak for the reconstruction software since there are no obvious pathologies. 

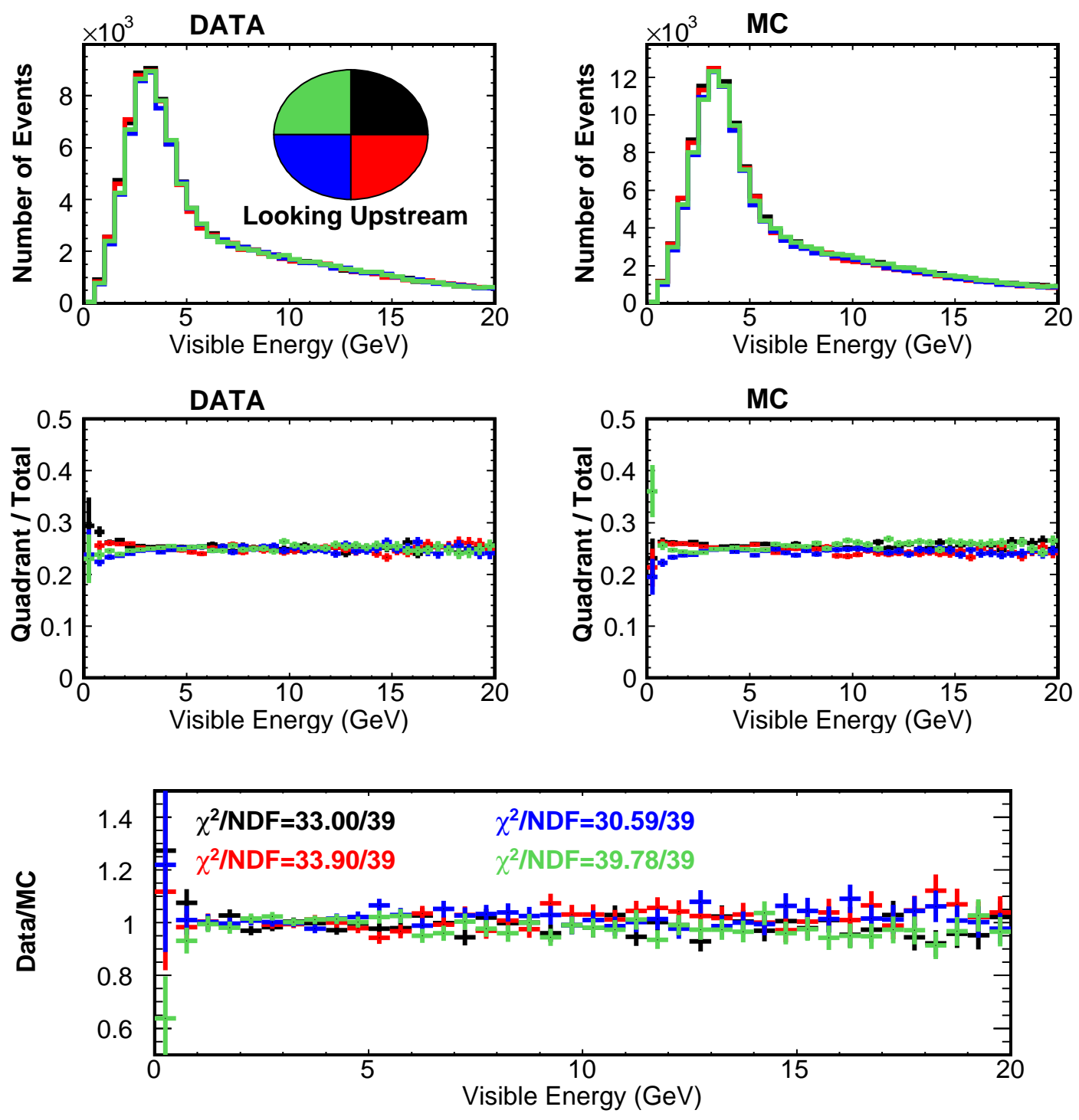

Figure 6.1: The charged current energy spectra for four different fiducial quadrants in the Near detector. The color of the spectrum corresponds to the color in the legend in the top left plot. The top left is data and the top right is Monte Carlo. The middle two plots are the ratios of each of the individual spectra divided by the total spectra. The bottom plot shows the ratio of these ratios with $\chi^{2} / \mathrm{ndf}$ superimposed from a fit to $f(x)=1$ 

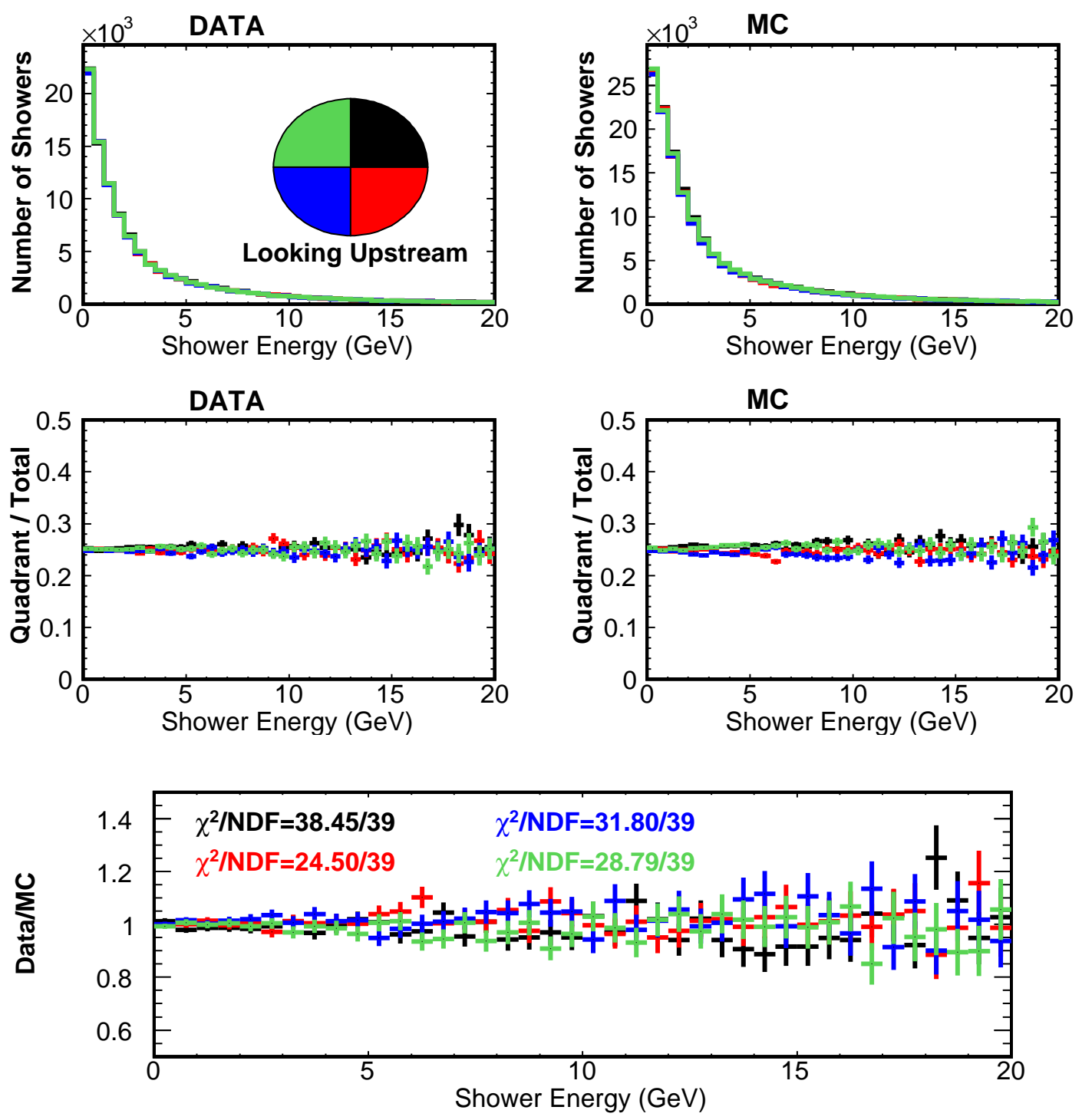

Figure 6.2: The charged current shower energy for four different fiducial quadrants in the Near detector. The color of the spectrum corresponds to the color in the legend in the top left plot. The top left is data and the top right is Monte Carlo. The middle two plots are the ratios of each of the individual spectra divided by the total spectra. The bottom plot shows the ratio of these ratios with $\chi^{2} / \mathrm{ndf}$ superimposed from a fit to $f(x)=1$ 

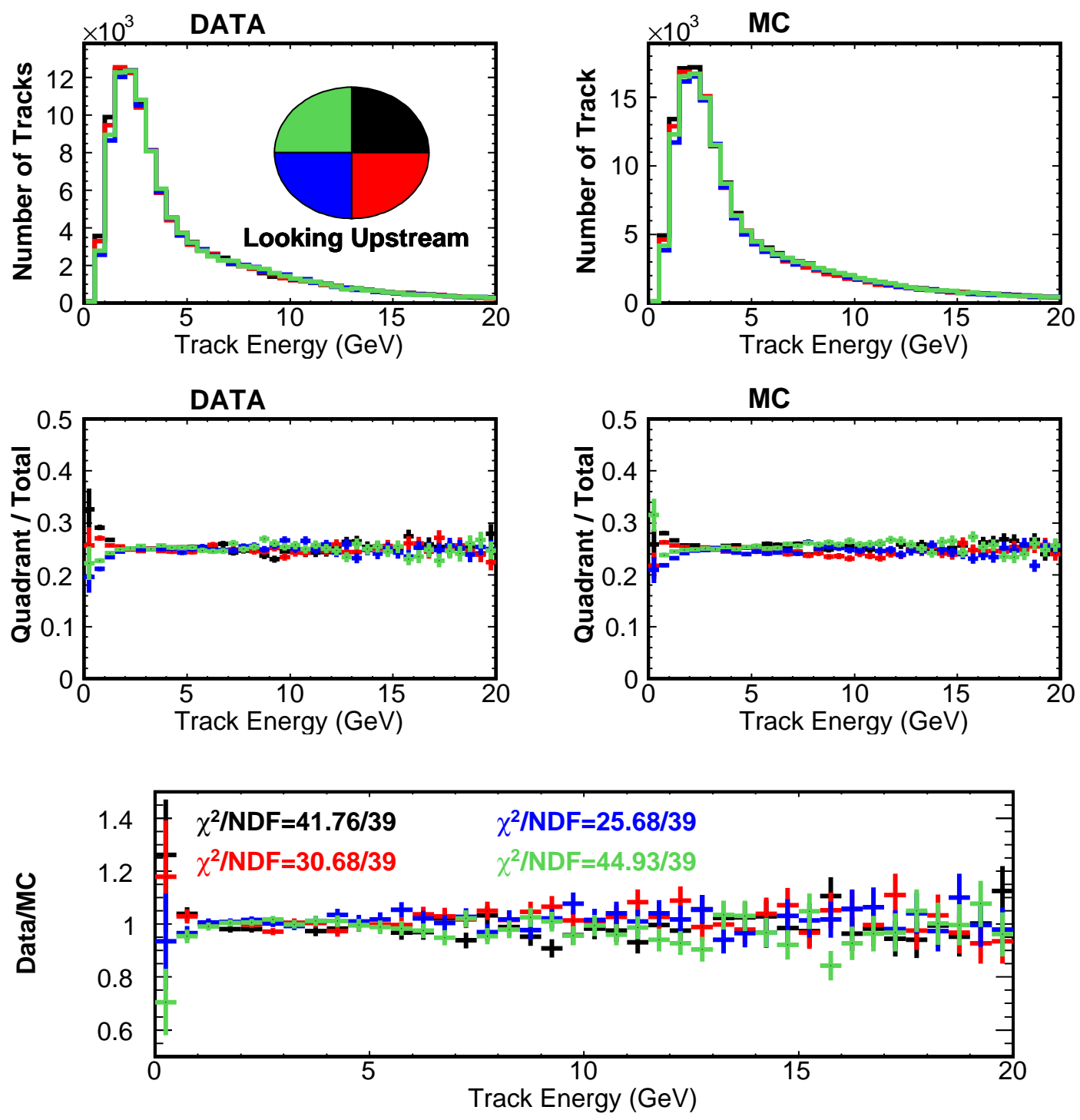

Figure 6.3: The charged current track energy for four different fiducial quadrants in the Near detector. The color of the spectrum corresponds to the color in the legend in the top left plot. The top left is data and the top right is Monte Carlo. The middle two plots are the ratios of each of the individual spectra divided by the total spectra. The bottom plot shows the ratio of these ratios with $\chi^{2} /$ ndf superimposed from a fit to $f(x)=1$ 

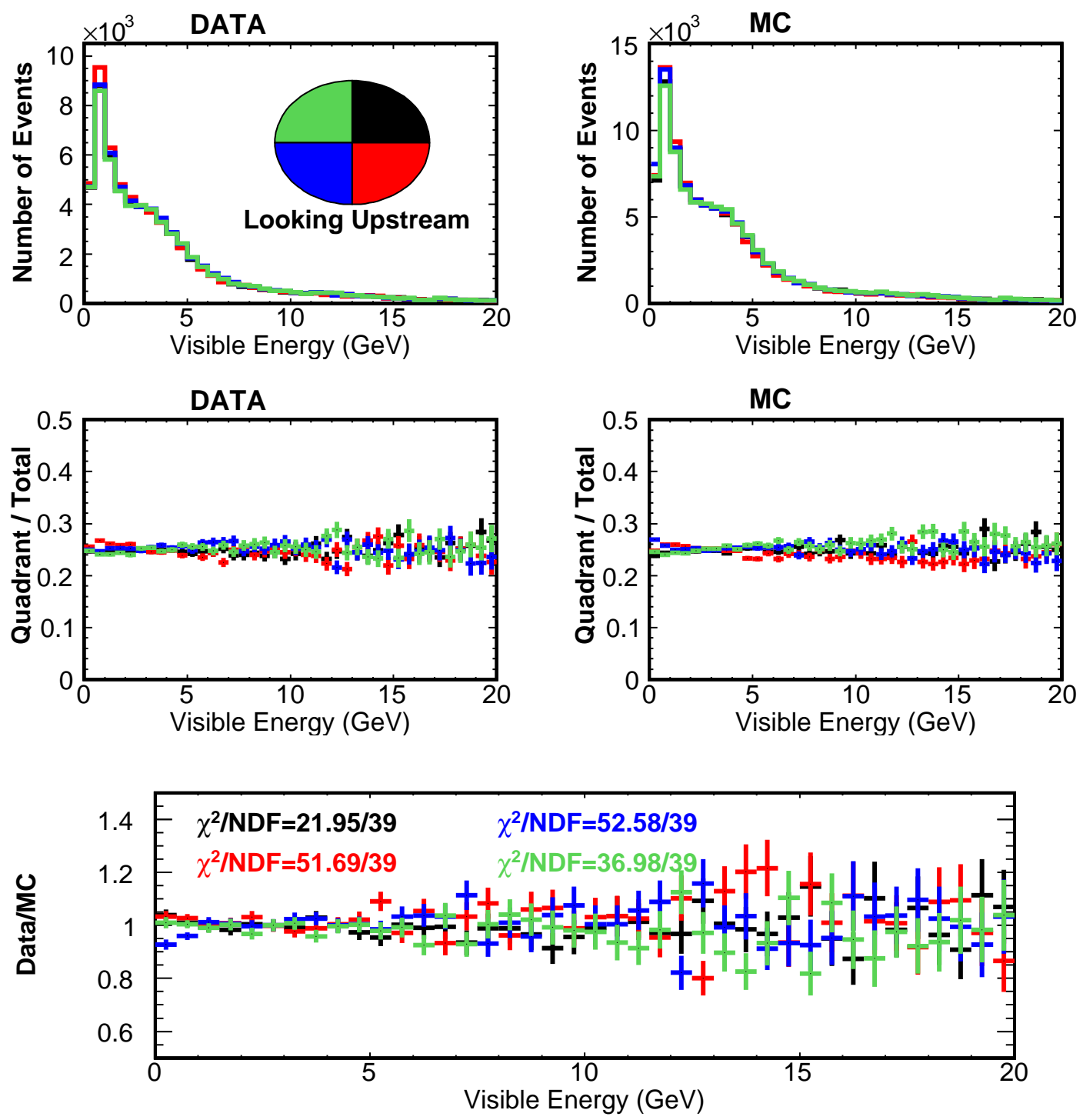

Figure 6.4: The neutral current visible energy spectra for four different fiducial quadrants in the Near detector. The color of the spectrum corresponds to the color in the legend in the top left plot. The top left is data and the top right is Monte Carlo. The middle two plots are the ratios of each of the individual spectra divided by the total spectra. The bottom plot shows the ratio of these ratios with $\chi^{2} / \mathrm{ndf}$ superimposed from a fit to $f(x)=1$ 

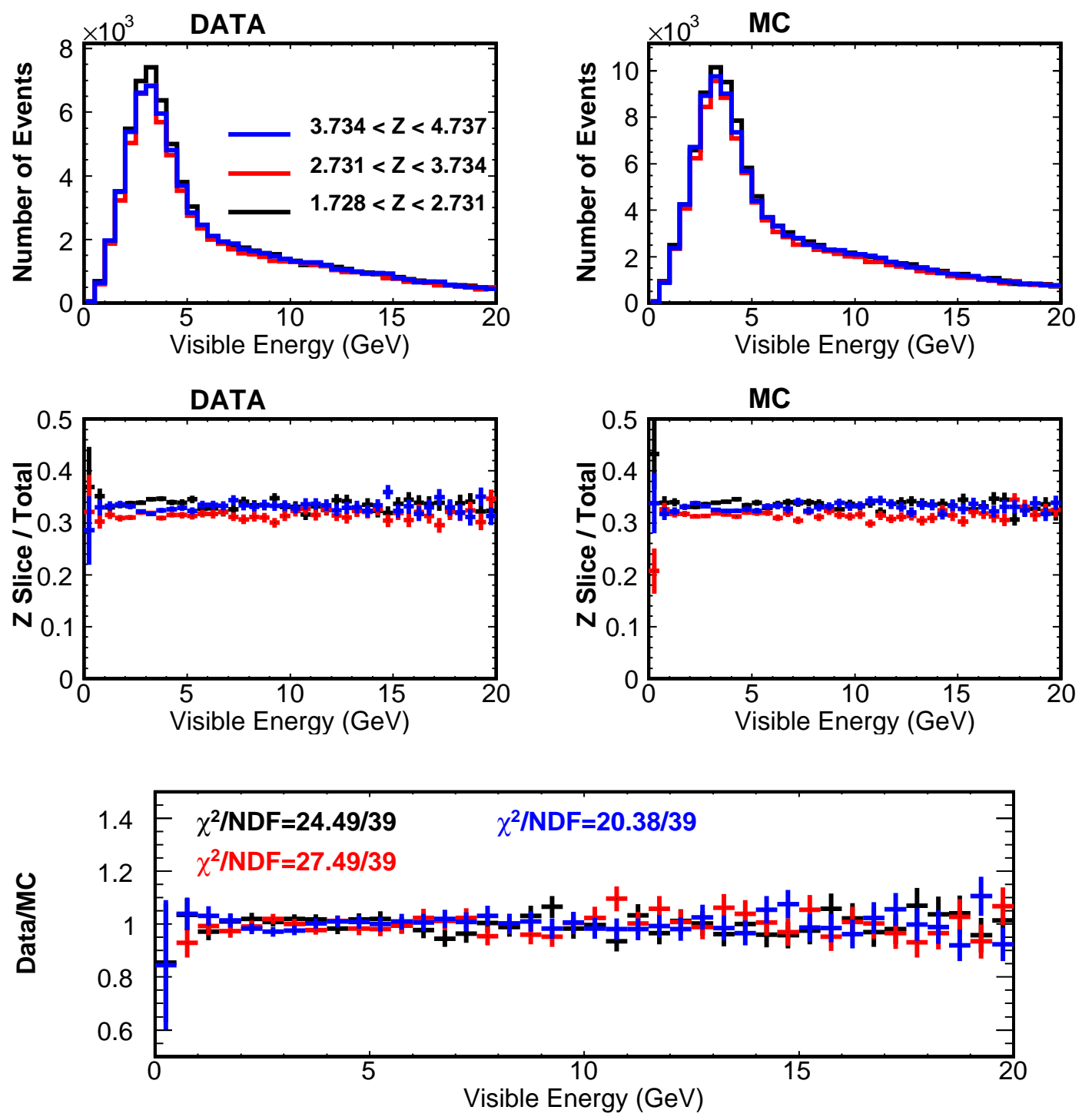

Figure 6.5: The charged current energy spectra for three different longitudinal segments in the Near detector fiducial volume. The color of the spectrum corresponds to the color in the legend in the top left plot. The top left is data and the top right is Monte Carlo. The middle two plots are the ratios of each of the individual spectra divided by the total spectra. The bottom plot shows the ratio of these ratios with $\chi^{2} /$ ndf superimposed from a fit to $f(x)=1$ 

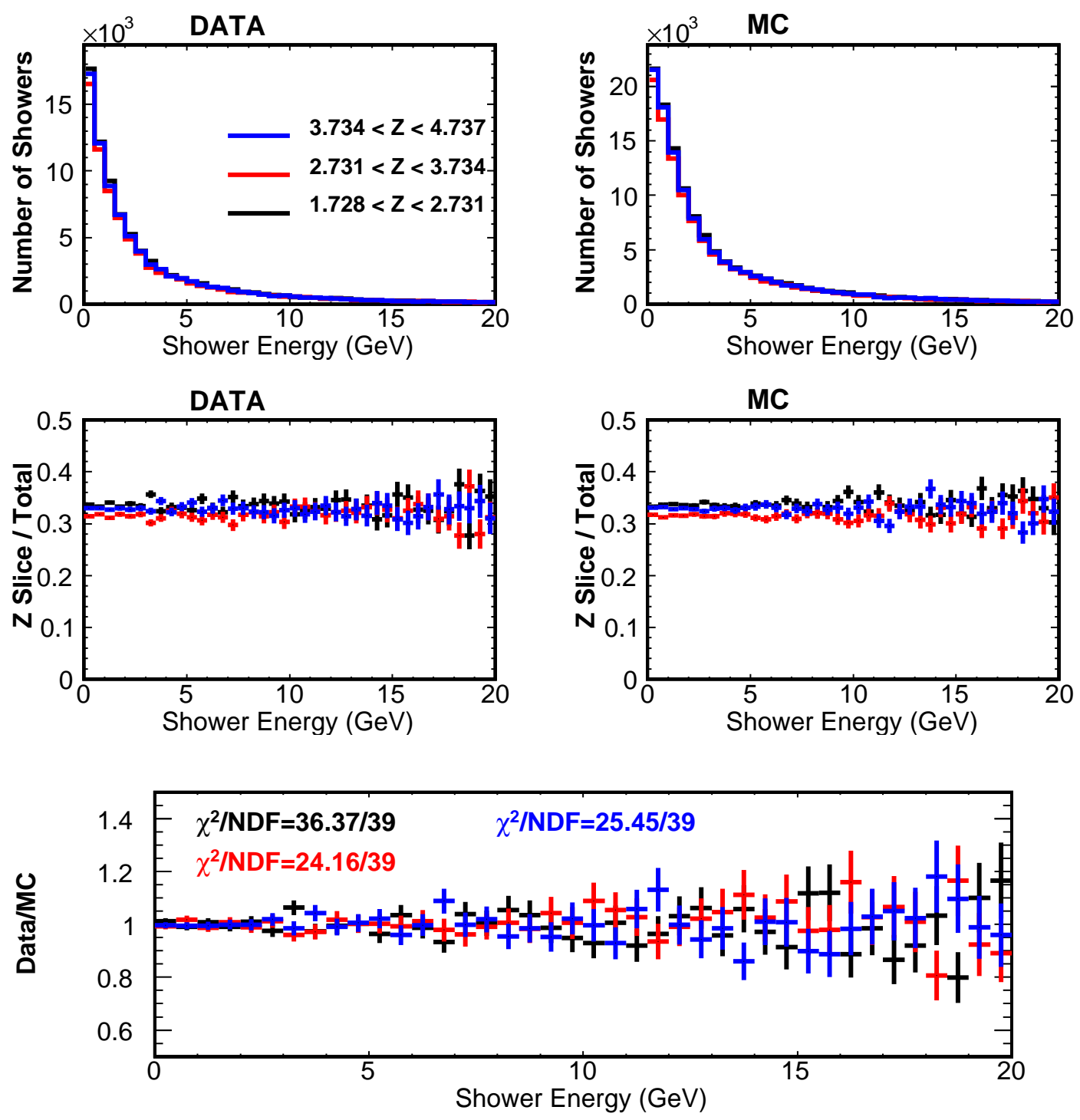

Figure 6.6: The charged current shower energy for three different longitudinal segments in the Near detector fiducial volume. The color of the spectrum corresponds to the color in the legend in the top left plot. The top left is data and the top right is Monte Carlo. The middle two plots are the ratios of each of the individual spectra divided by the total spectra. The bottom plot shows the ratio of these ratios with $\chi^{2} /$ ndf superimposed from a fit to $f(x)=1$ 

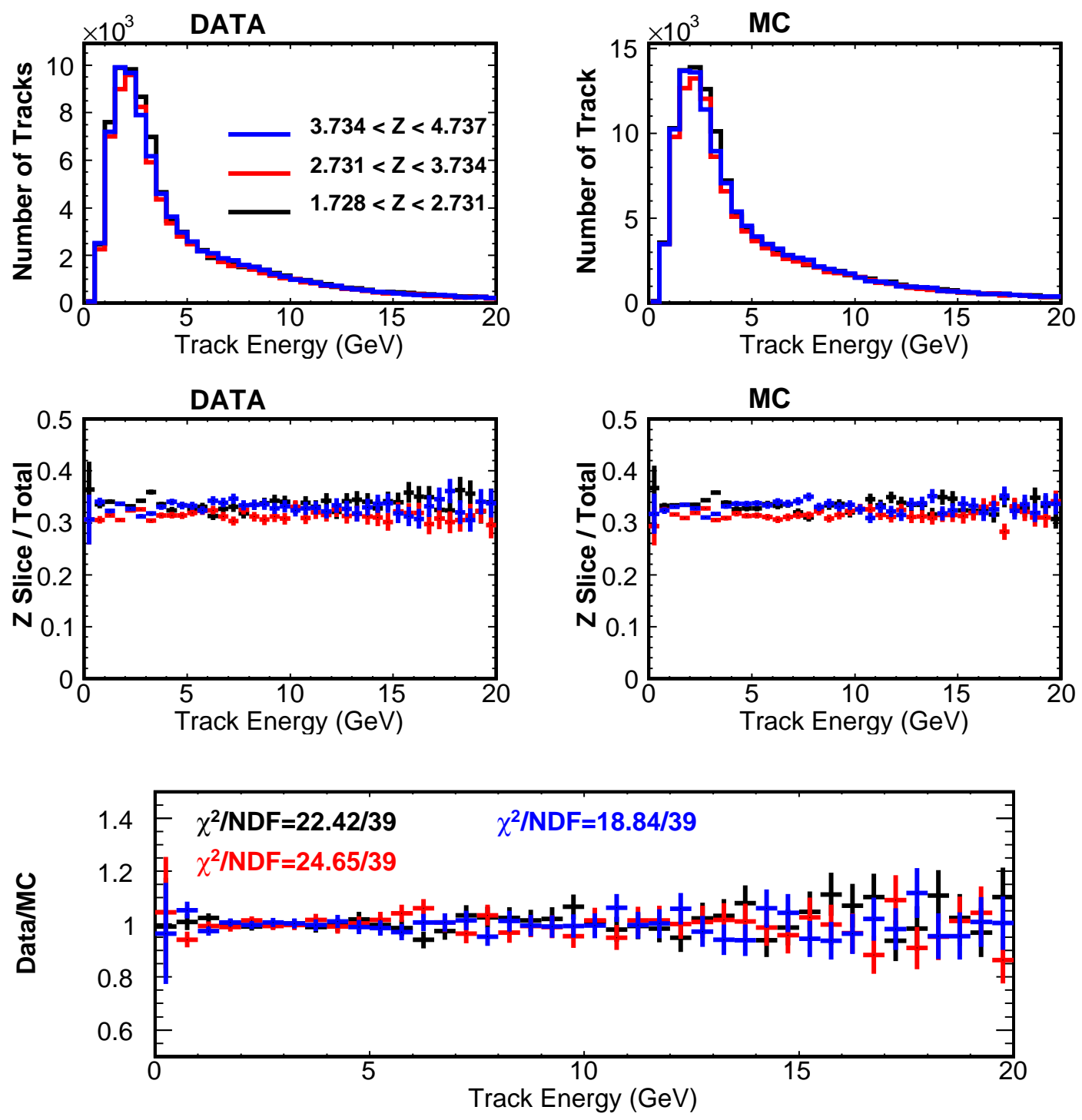

Figure 6.7: The charged current track energy for three different longitudinal segments in the Near detector fiducial volume. The color of the spectrum corresponds to the color in the legend in the top left plot. The top left is data and the top right is Monte Carlo. The middle two plots are the ratios of each of the individual spectra divided by the total spectra. The bottom plot shows the ratio of these ratios with $\chi^{2} /$ ndf superimposed from a fit to $f(x)=1$ 

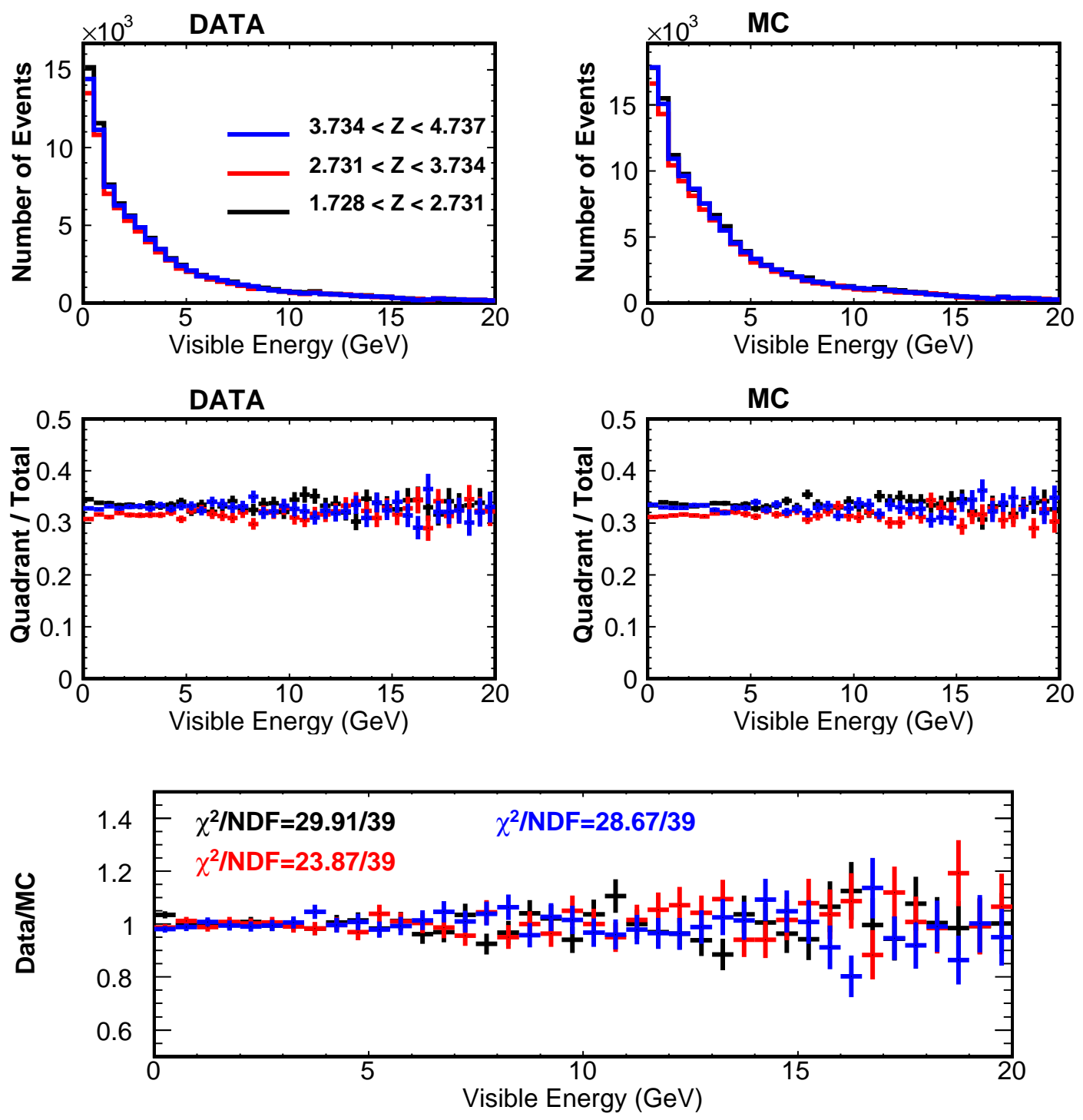

Figure 6.8: The neutral current visible energy spectra for three different longitudinal segments in the Near detector fiducial volume. The color of the spectrum corresponds to the color in the legend in the top left plot. The top left is data and the top right is Monte Carlo. The middle two plots are the ratios of each of the individual spectra divided by the total spectra. The bottom plot shows the ratio of these ratios with $\chi^{2} /$ ndf superimposed from a fit to $f(x)=1$ 

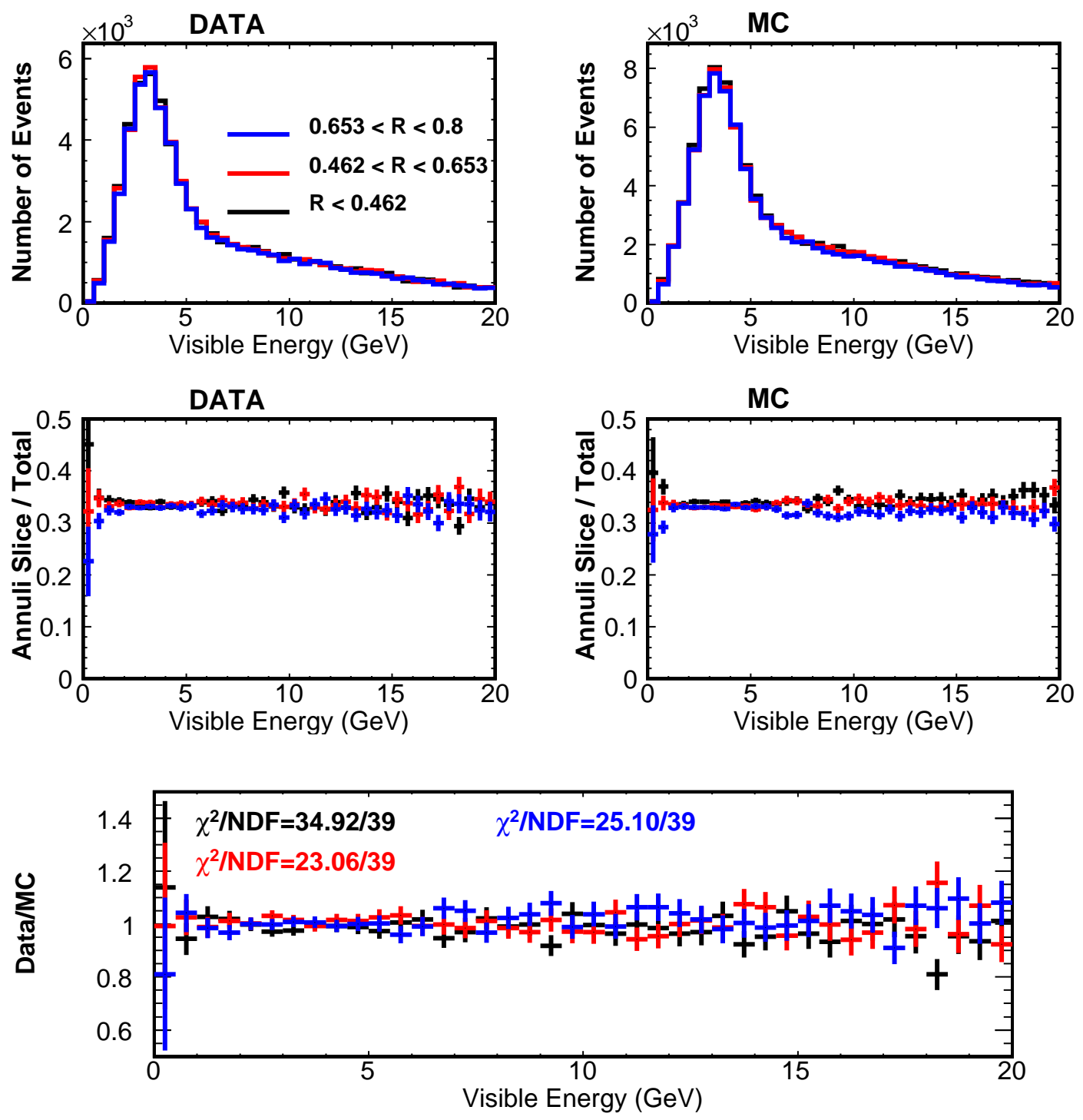

Figure 6.9: The charged current energy spectra for three radial annuli in the Near detector fiducial volume. The color of the spectrum corresponds to the color in the legend in the top left plot. The top left is data and the top right is Monte Carlo. The middle two plots are the ratios of each of the individual spectra divided by the total spectra. The bottom plot shows the ratio of these ratios with $\chi^{2} /$ ndf superimposed from a fit to $f(x)=1$ 

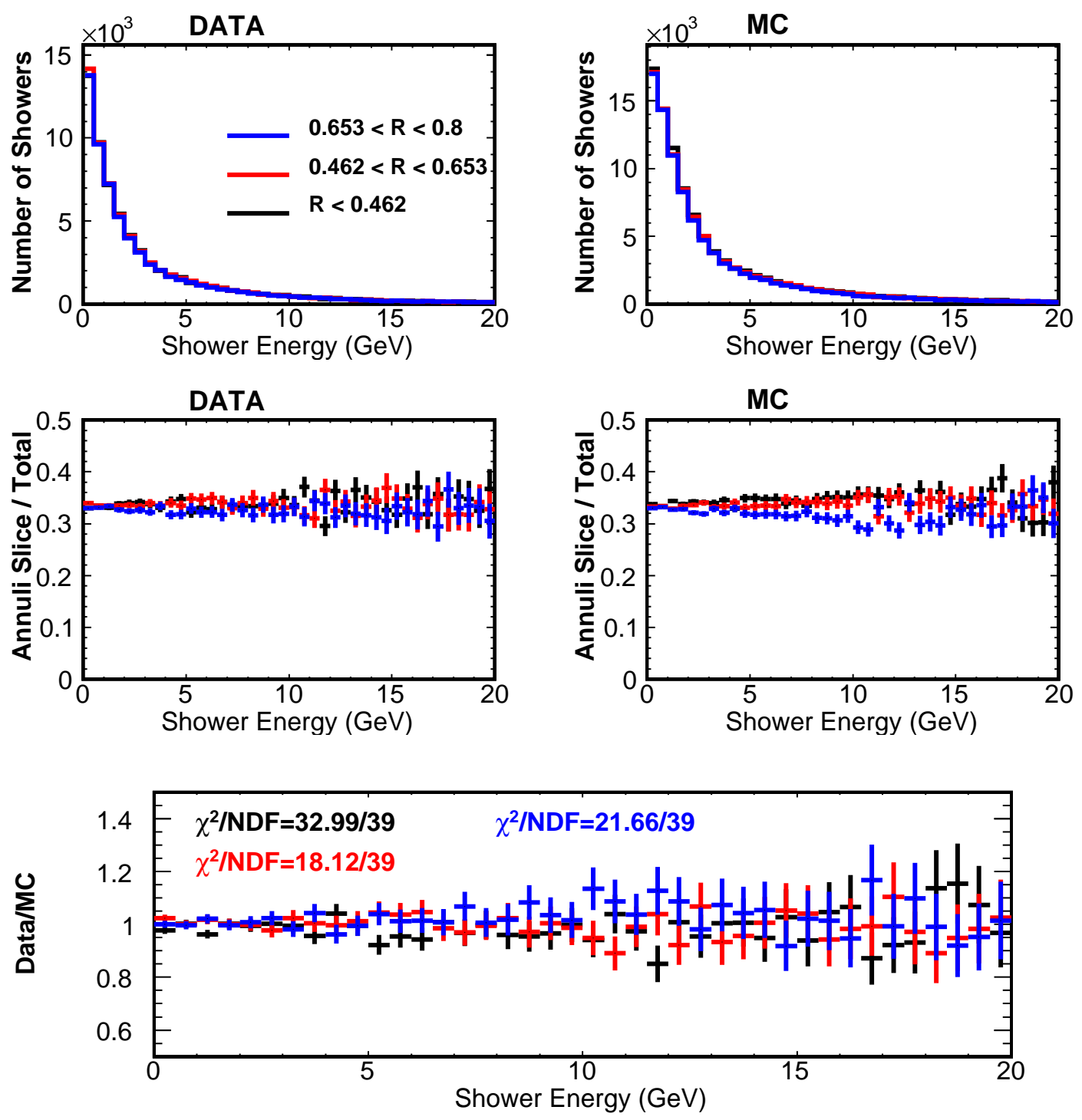

Figure 6.10: The charged current shower energy for three radial annuli in the Near detector fiducial volume. The color of the spectrum corresponds to the color in the legend in the top left plot. The top left is data and the top right is Monte Carlo. The middle two plots are the ratios of each of the individual spectra divided by the total spectra. The bottom plot shows the ratio of these ratios with $\chi^{2} /$ ndf superimposed from a fit to $f(x)=1$ 

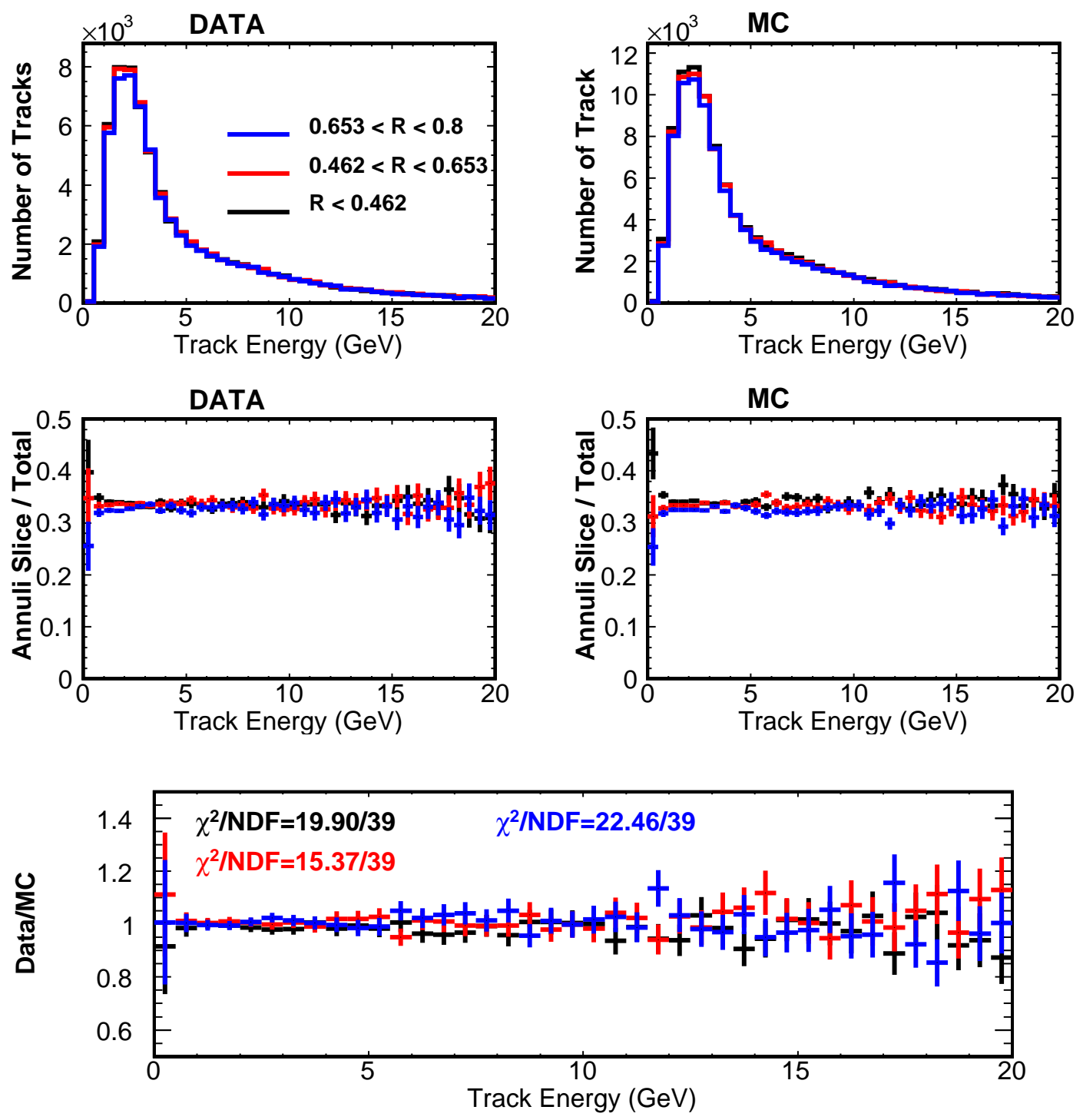

Figure 6.11: The charged current track energy for three radial annuli in the Near detector fiducial volume. The color of the spectrum corresponds to the color in the legend in the top left plot. The top left is data and the top right is Monte Carlo. The middle two plots are the ratios of each of the individual spectra divided by the total spectra. The bottom plot shows the ratio of these ratios with $\chi^{2} /$ ndf superimposed from a fit to $f(x)=1$ 

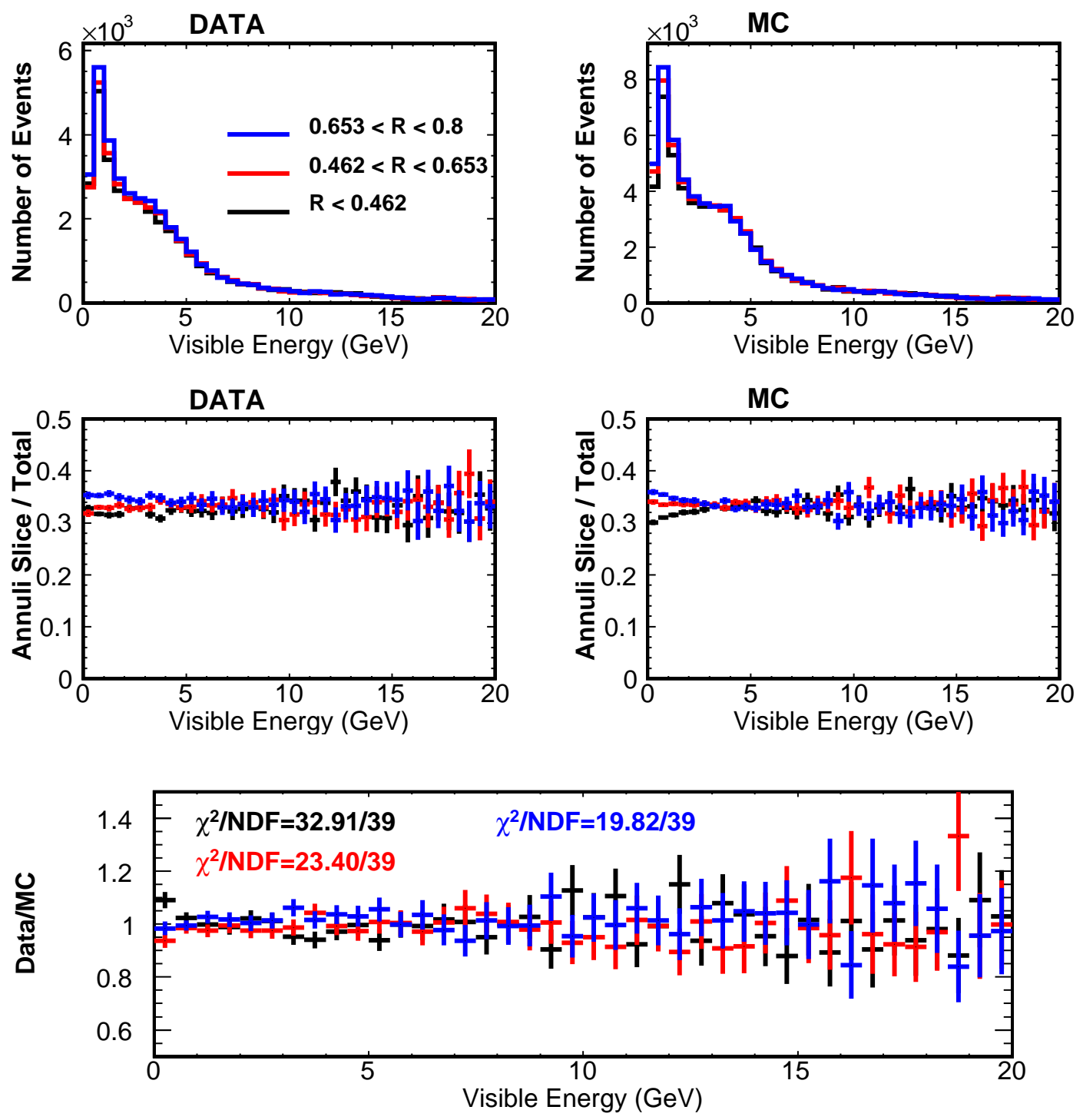

Figure 6.12: The neutral current visible energy spectra for three radial annuli in the Near detector fiducial volume. The color of the spectrum corresponds to the color in the legend in the top left plot. The top left is data and the top right is Monte Carlo. The middle two plots are the ratios of each of the individual spectra divided by the total spectra. The bottom plot shows the ratio of these ratios with $\chi^{2} / \mathrm{ndf}$ superimposed from a fit to $f(x)=1$ 


\subsection{Intensity Studies}

We present various plots illustrating the effect of the relatively high intensity NuMI beam on the event reconstruction in the MINOS Near detector. Comparisons of various classes of events taken at the nominal high intensity of $2.5 \times 10^{13}$ protons per pulse (ppp) and compared with data taken in special runs at intensities of about $2 \times 10^{12}$ (low intensity) and $6 \times 10^{12} \mathrm{ppp}$ (medium intensity). We use a total of $6.05 \times 10^{18}$ POT for the high intensity, $5.24 \times 10^{17}$ POT for the medium intensity and $2.63 \times 10^{17}$ POT for the low intensity data.

We first show in Figures 6.13 the average number of reconstructed slices and events as a function of beam intensity. These plots show a linear relationship between the two quantities. This linear relationship indicates that the Near detector is able to measure individual neutrino interactions with a negligible background.

In Figures 6.14 through 6.17 we plot several variables measured in the Near detector for the three different intensities listed above. We always normalize each spectra by total protons on target to the low intensity sample since it has the least amount of statistics.

We organize these plots into different categories of variables. Figure 6.14 deals with event specific quantities. We plot the total event energy for charged current events in units of both $\mathrm{GeV}$ and $\mathrm{ADC}$ counts, along with the reconstructed $\mathrm{y}=$ Hadronic Energy / Total Energy and event length. In Figures 6.15 through 6.16 quantities associated with reconstructed showers which include the shower energy, shower length, number of reconstructed strips, shower pulse height per plane and per strip, and the lateral widths of showers in both $U$ and V views. Figure 6.17 has track related quantities, including the track pulse height per plane, track momentum as determined from range, and track momentum from curvature. The Slight differences in all these plots are related to a slightly higher efficiency at lower intensities because the reconstruction has a cleaner environment in which to locate tracks and showers. 
In Figures 6.18 through 6.37 we show that important variables in the Near detector, though changing as a function of intensity, are well modeling by the Monte Carlo. These figures have a format similar in that to the stability studies in Figures 6.1 through 6.12 . We take Figure 6.18 as an example. The top left plots shows the energy from charged current events for Near detector data. The red spectra is at low intensity and the black is at high intensity. The top right plot is the same except it is Monte Carlo. The following two plots are the ratios of the high intensity to low intensity data in the middle left, and the same for Monte Carlo on the middle right. The bottom plot is the ratio of the previous two ratios. If data follows Monte Carlo, the bottom plot should be a flat line at one. Superimposed on this plot is $\chi^{2}$ per number of degrees of freedom for a fit between the ratio and $f(x)=1$. For all cases, the ratio is statistically consistent with being equal to one.

All the results of the intensity studies confirm that not only does our Monte Carlo model the effects of beam intensity properly, but that the slicing algorithm does not introduce any biases into our data. 

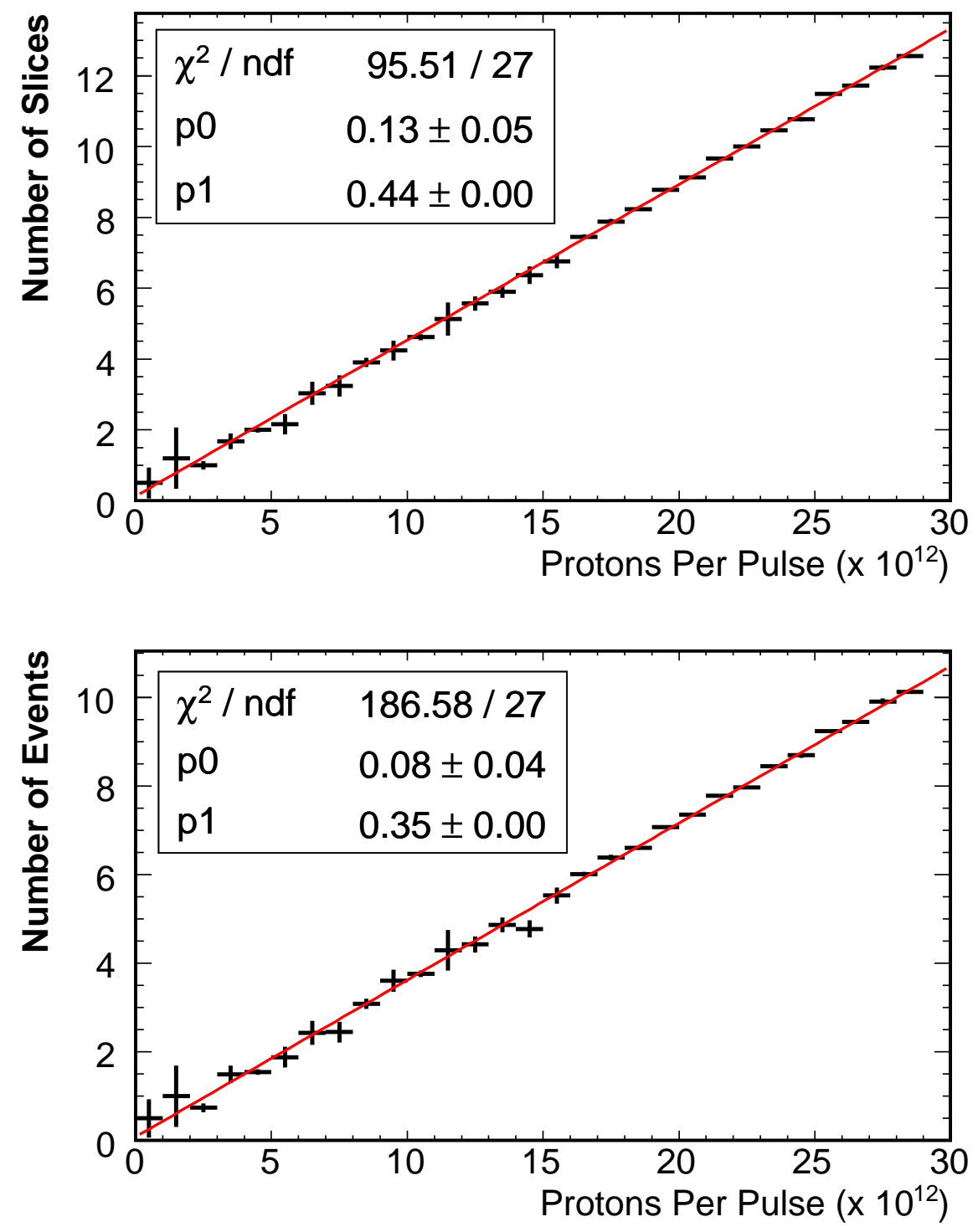

Figure 6.13: A plot of beam intensity $\left(\times 10^{12}\right.$ protons per pulse $)$ versus the average number of reconstructed slices in the top plot and the average number of reconstructed events in the bottom plot.) 


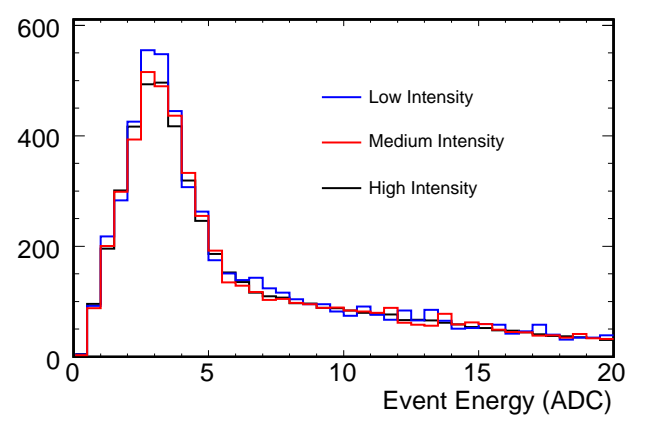

(a)

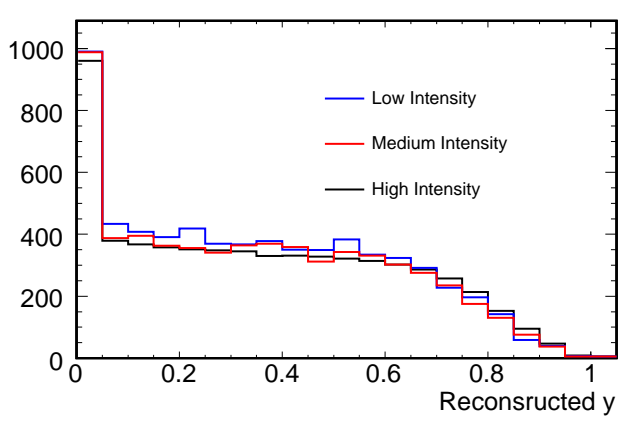

(c)

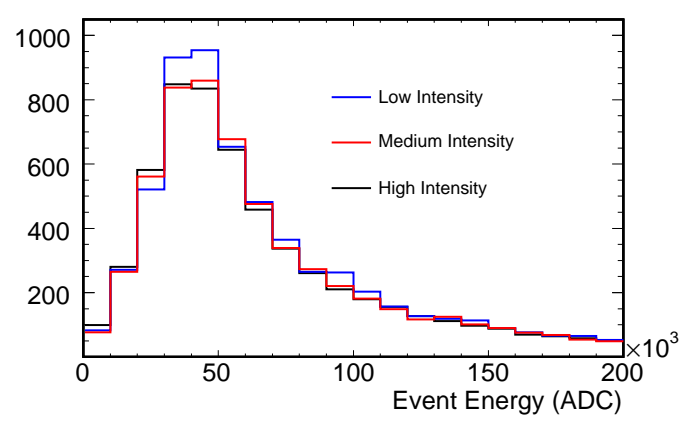

(b)

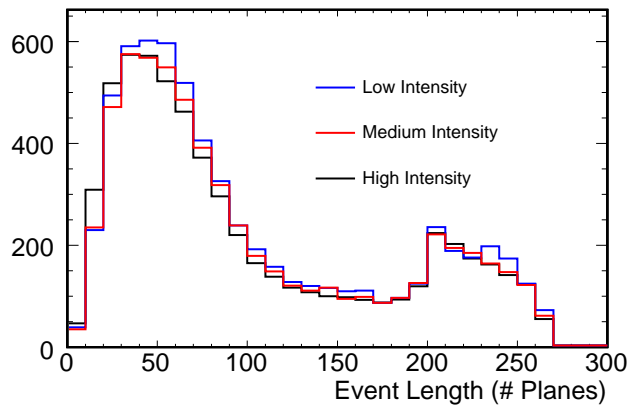

(d)

Figure 6.14: (a) The total neutrino energy for charged current events at three different beam intensities. (b) Charged current event energy in ADC counts at three different beam intensities. (c) The reconstructed y distribution for charged current events at three different beam intensities. (d) The event length in number of planes at three different beam intensities. 


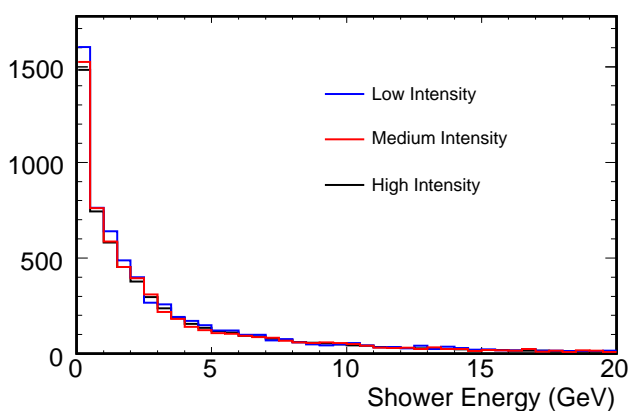

(a)

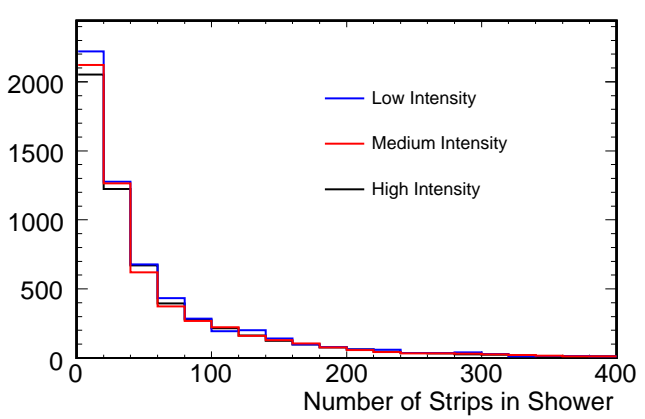

(c)

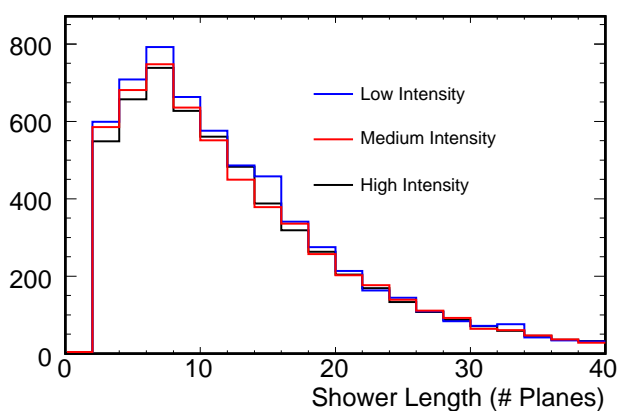

(b)

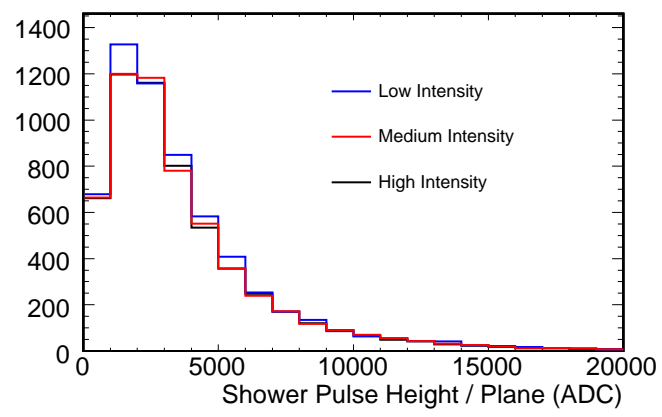

(d)

Figure 6.15: (a) Total shower energies at three different intensities. (b) The shower length in number of planes at three different beam intensities. (c) The number of reconstructed strips in a shower at three different beam intensities. (d) The average shower pulse height per plane in ADC counts for three different beam intensities. 


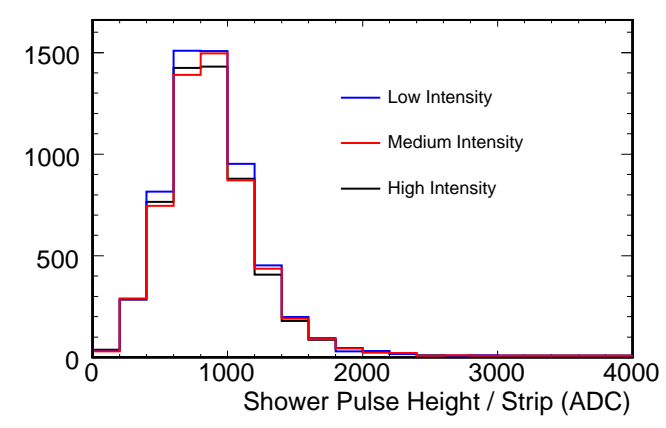

(a)

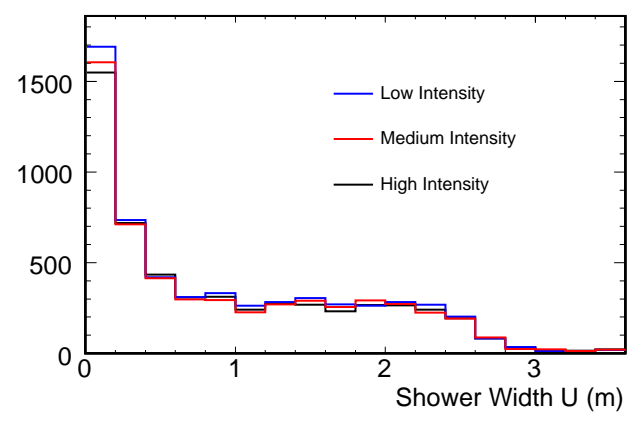

(b)

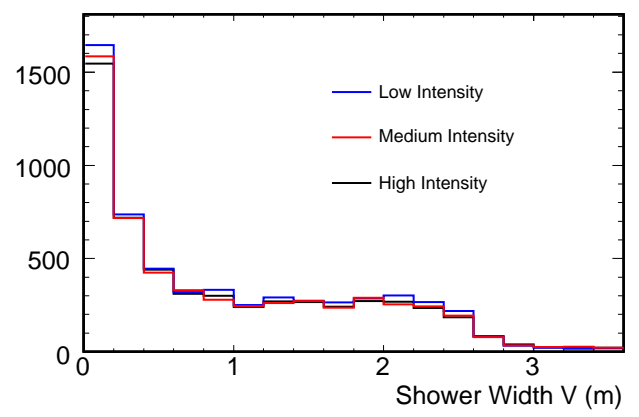

(c)

Figure 6.16: (a) The average shower pulse height per strip in ADC counts for three different beam intensities. (b) The lateral width of showers in the $\mathrm{U}$ view for three different beam intensities. (c) The lateral width of showers in the $\mathrm{V}$ view for three different beam intensities. 


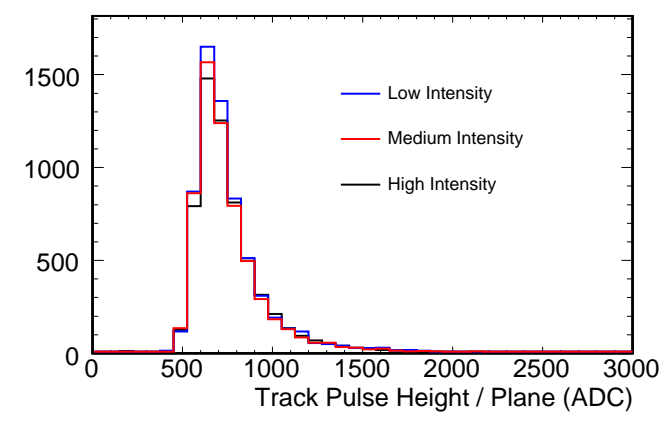

(a)

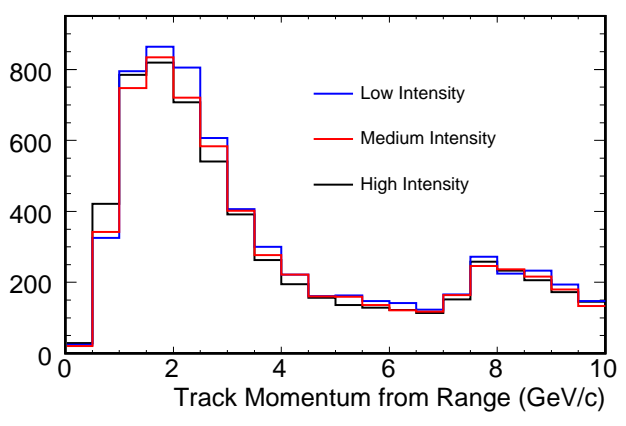

(b)

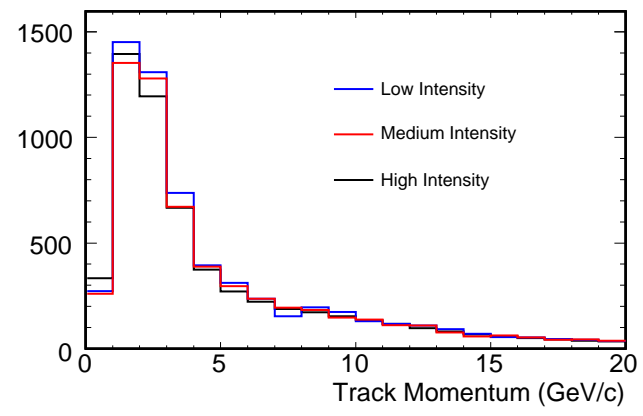

(c)

Figure 6.17: (a) The average track pulse height per plane in ADC counts for three different beam intensities. (b) The track momentum as determined from range at three different beam intensities. (c) The track momentum from curvature at three different beam intensities. 

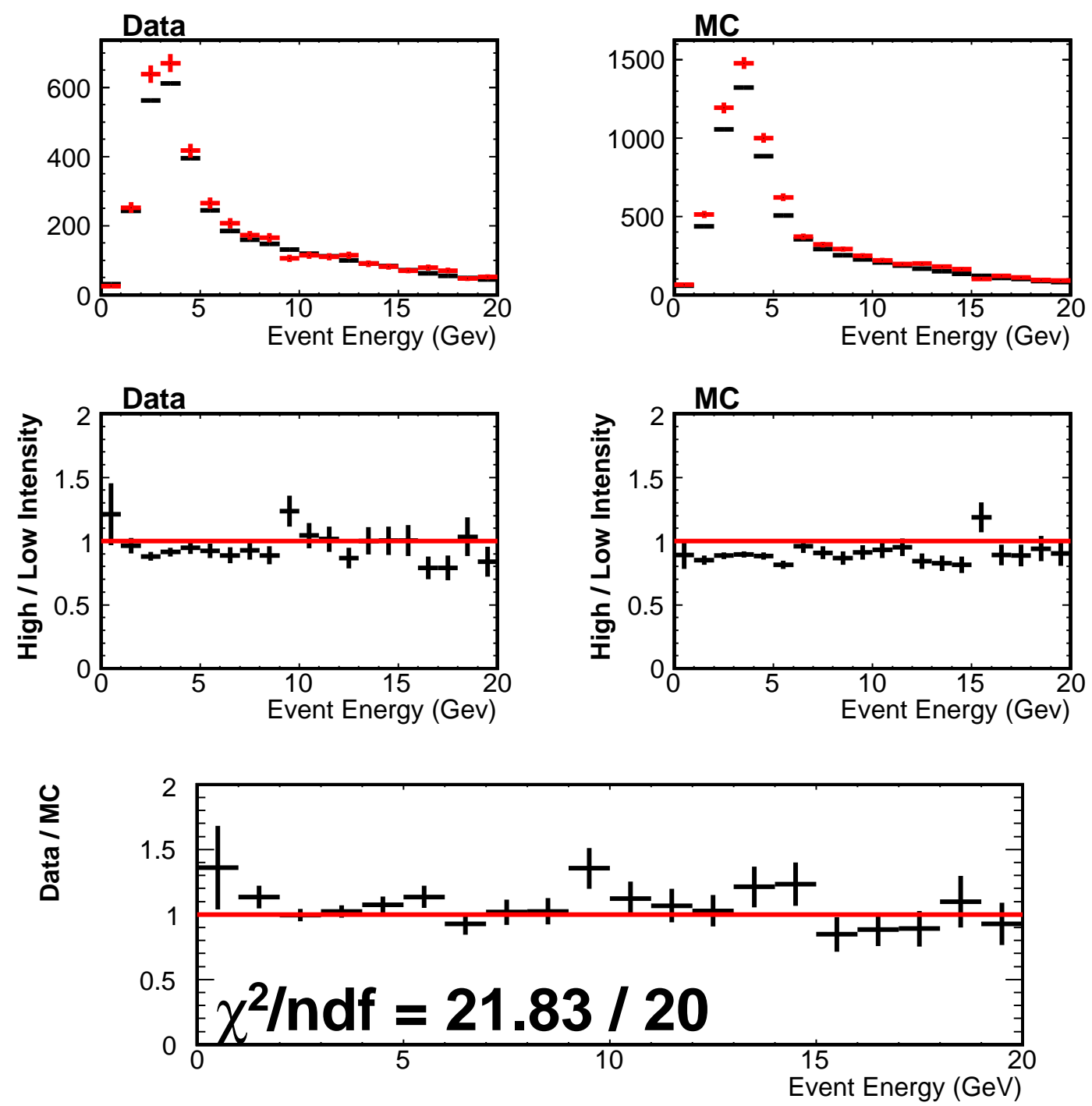

Figure 6.18: Comparison of the charged current total energy for low and high intensities. 

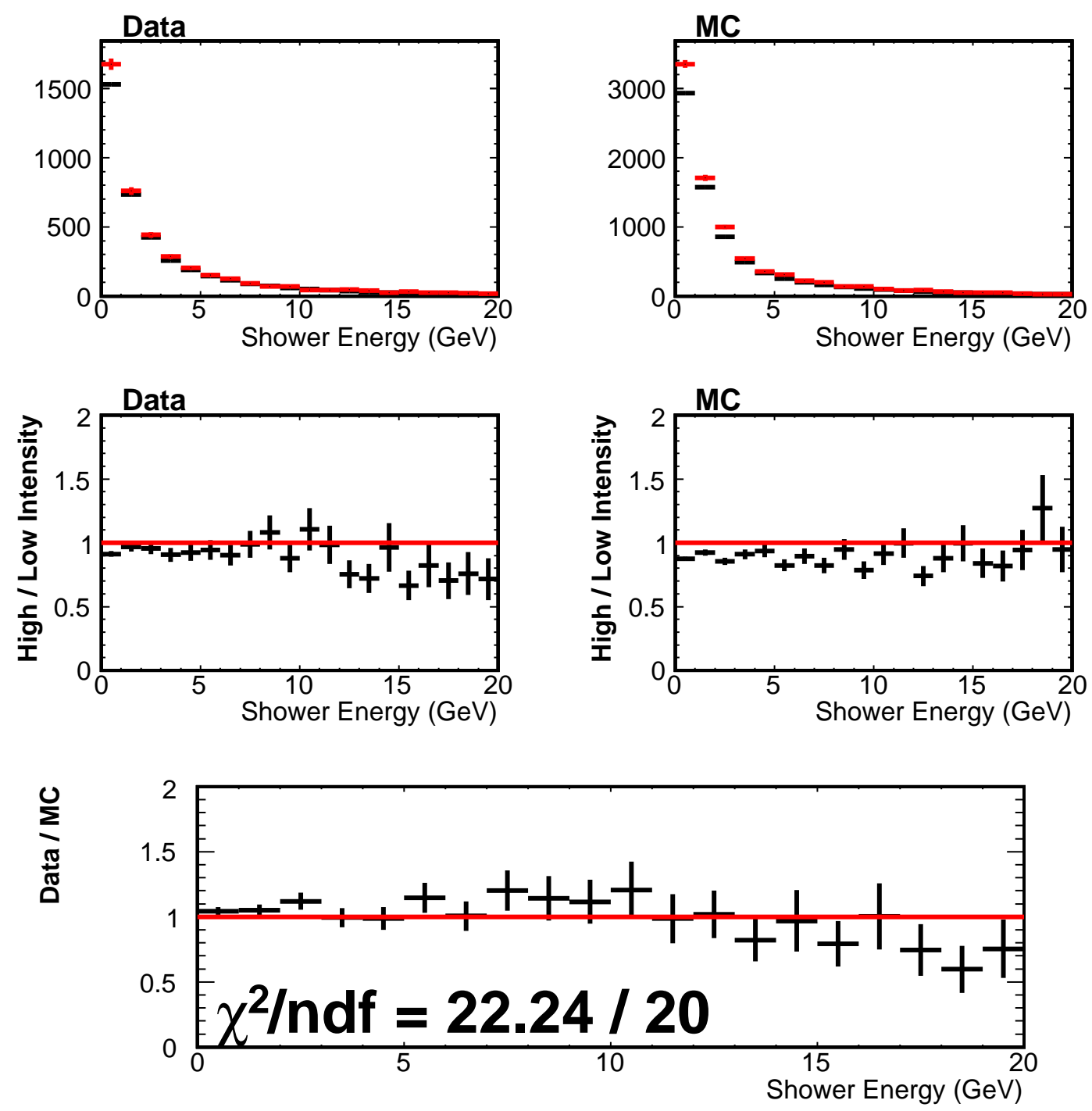

Figure 6.19: Comparison of the charged current shower energy for low and high intensities. 

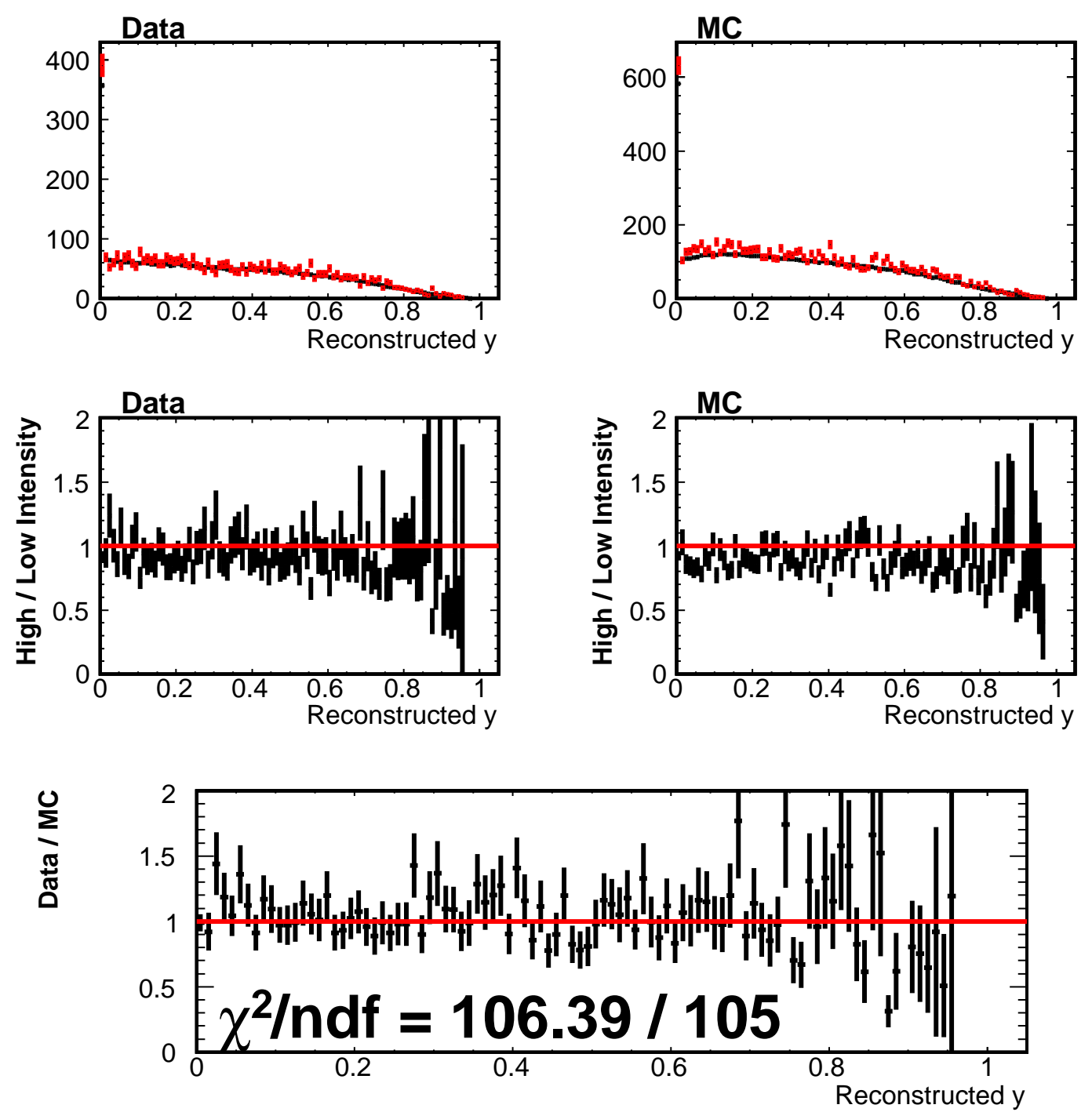

Figure 6.20: Comparison of the charged current $y_{b j}$ distribution for low and high intensities, where $y_{b j}$ is defined as the fraction of the neutrino energy deposited by hadronic contributions. 

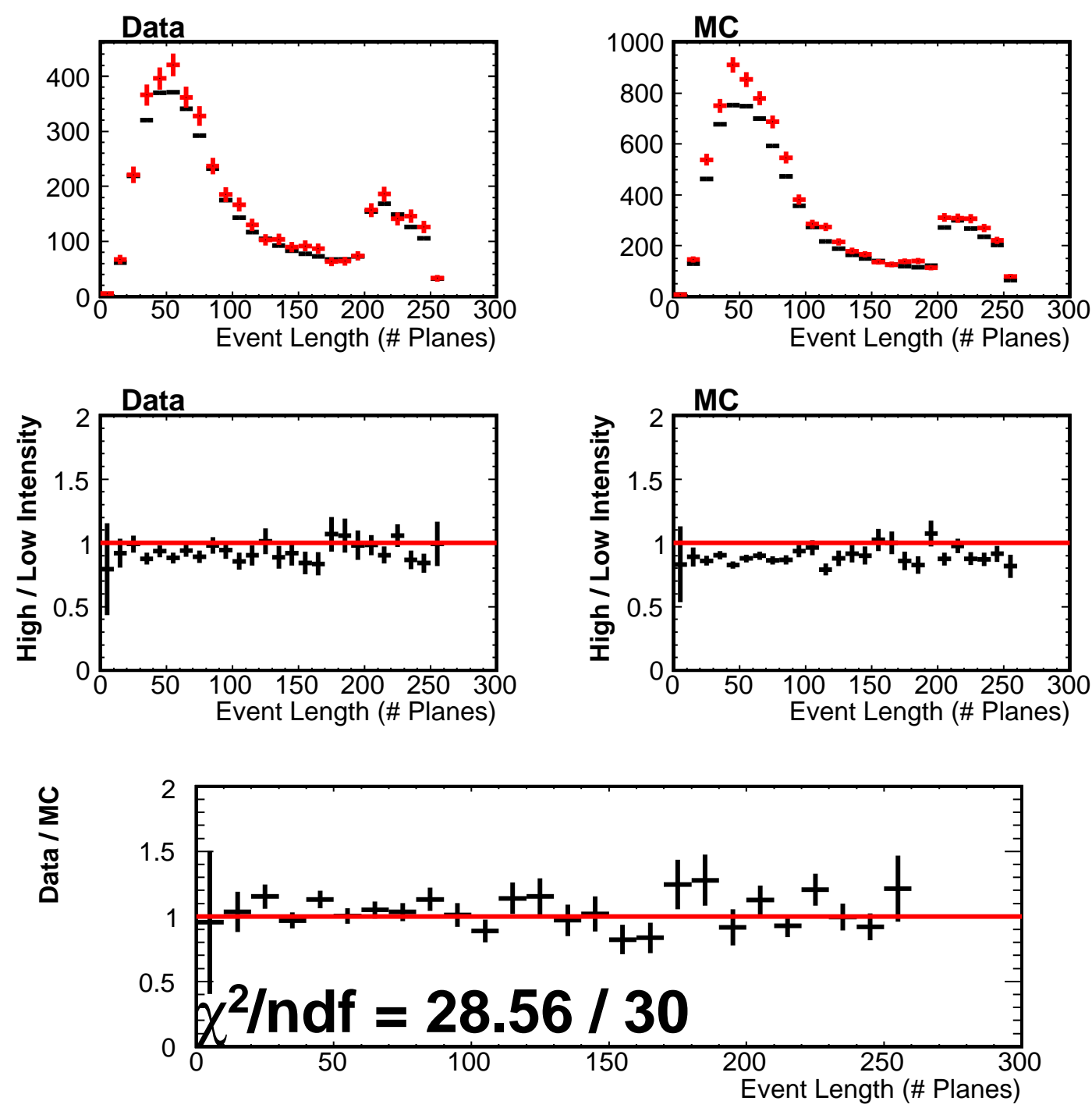

Figure 6.21: Comparison of the charged current event length for low and high intensities. 

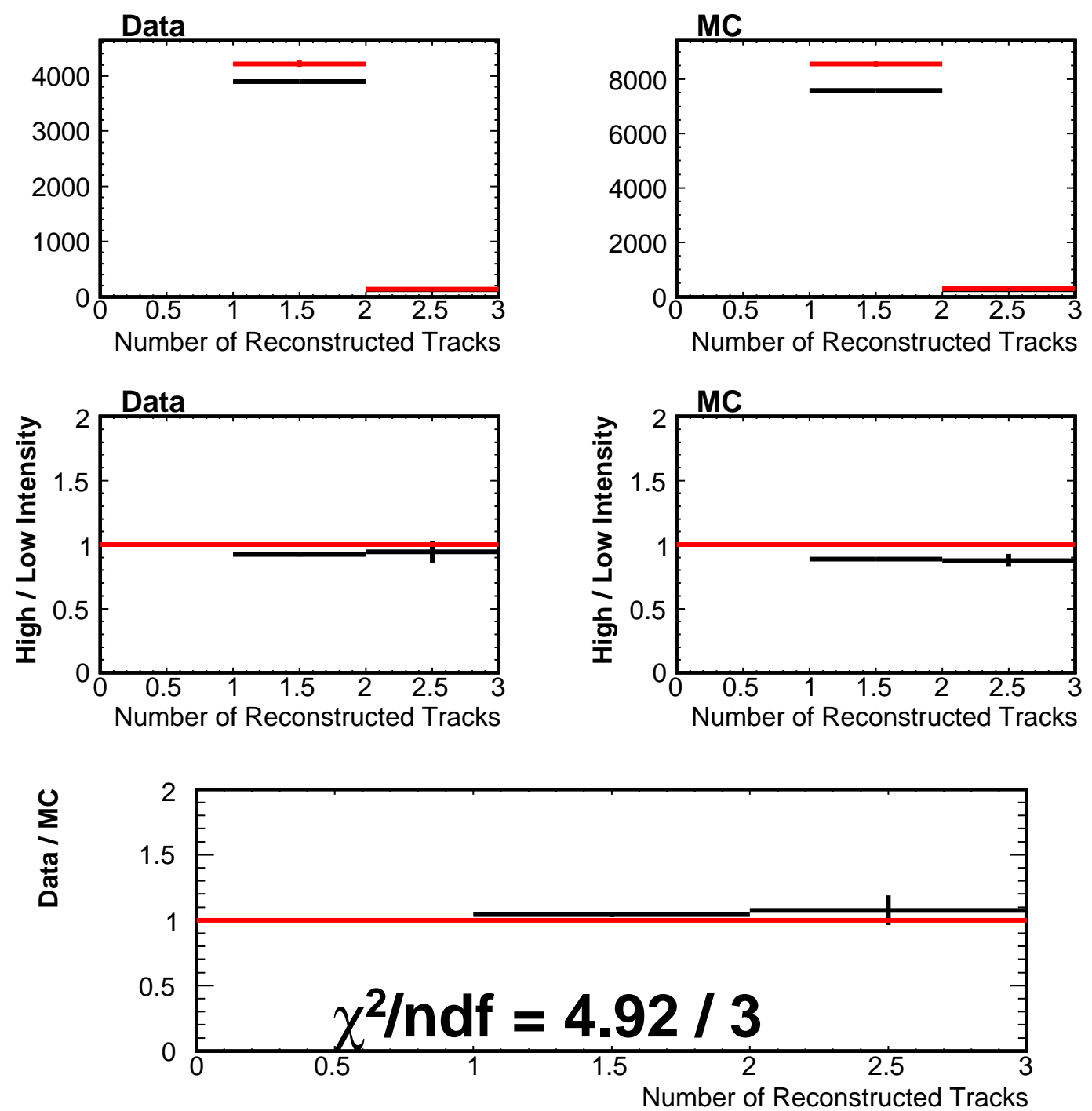

Figure 6.22: Comparison of the number of reconstructed tracks in charged current events for low and high intensities. 

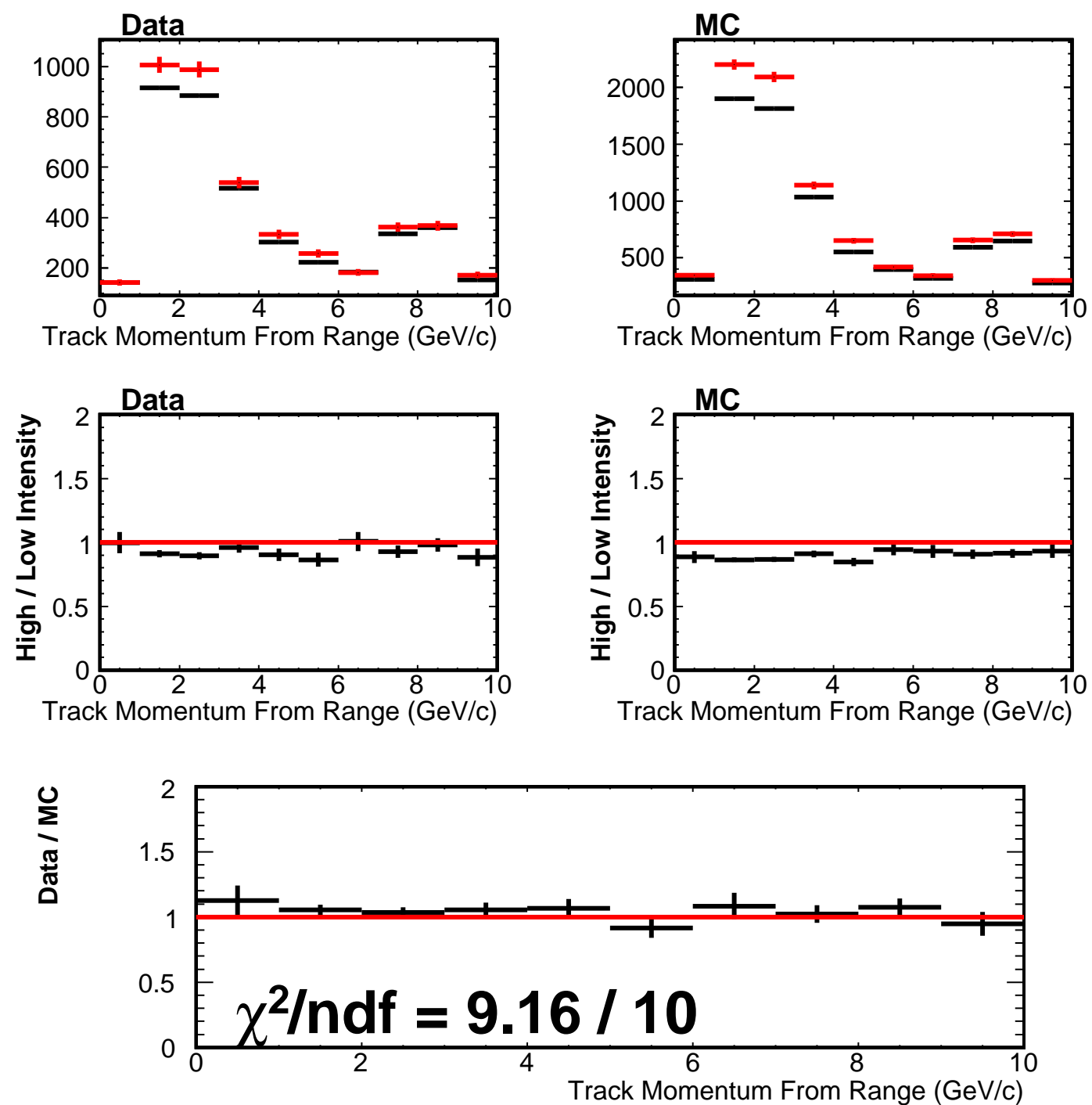

Figure 6.23: Comparison of the charged current track momentum determined from range for low and high intensities. 

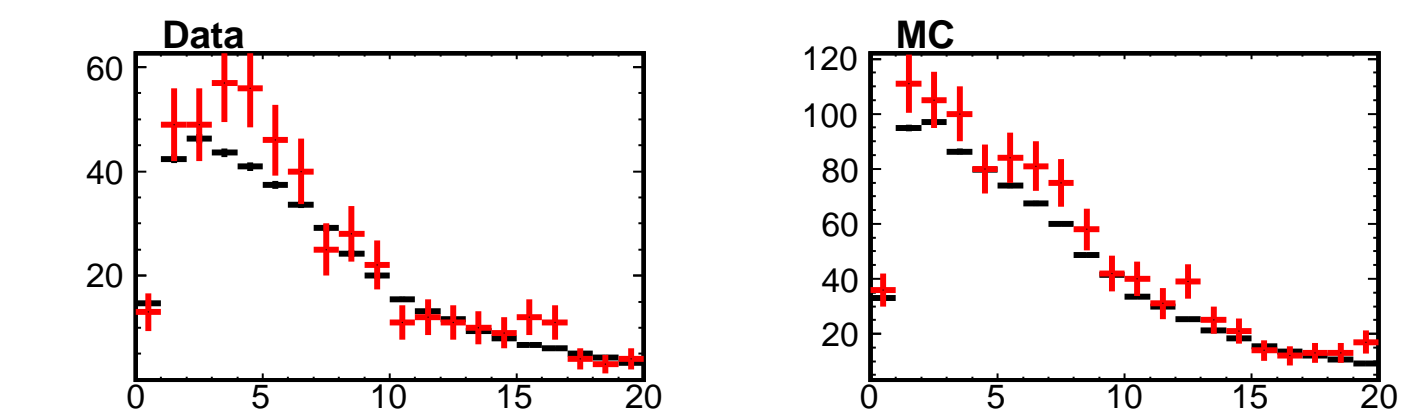

Track Momentum From Curvature $(\mathrm{GeV} / \mathrm{c})$

Track Momentum From Curvature $(\mathrm{GeV} / \mathrm{c})$

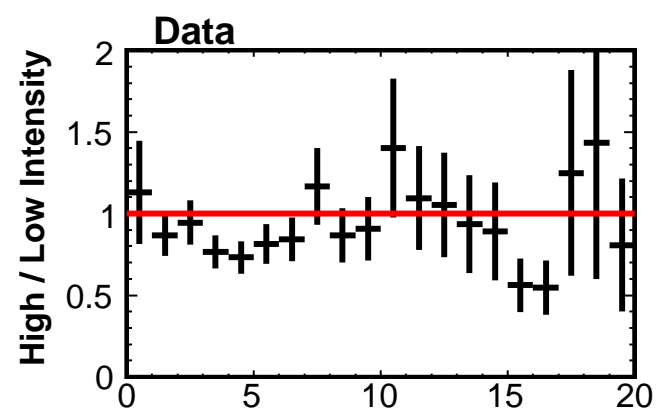

Track Momentum From Curvature $(\mathrm{GeV} / \mathrm{c})$

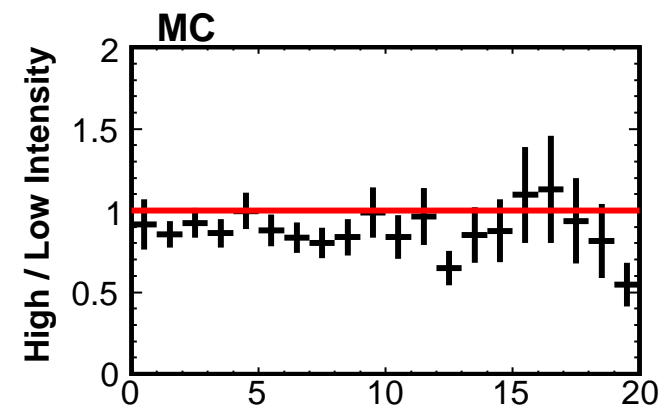

Track Momentum From Curvature (GeV/c)

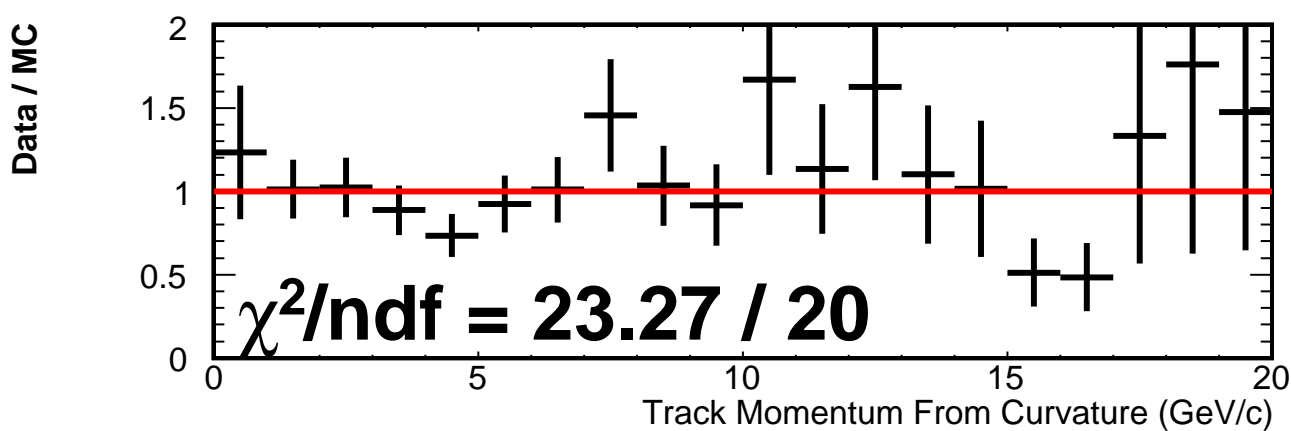

Figure 6.24: Comparison of the charged current track momentum from curvature for low and high intensities. 

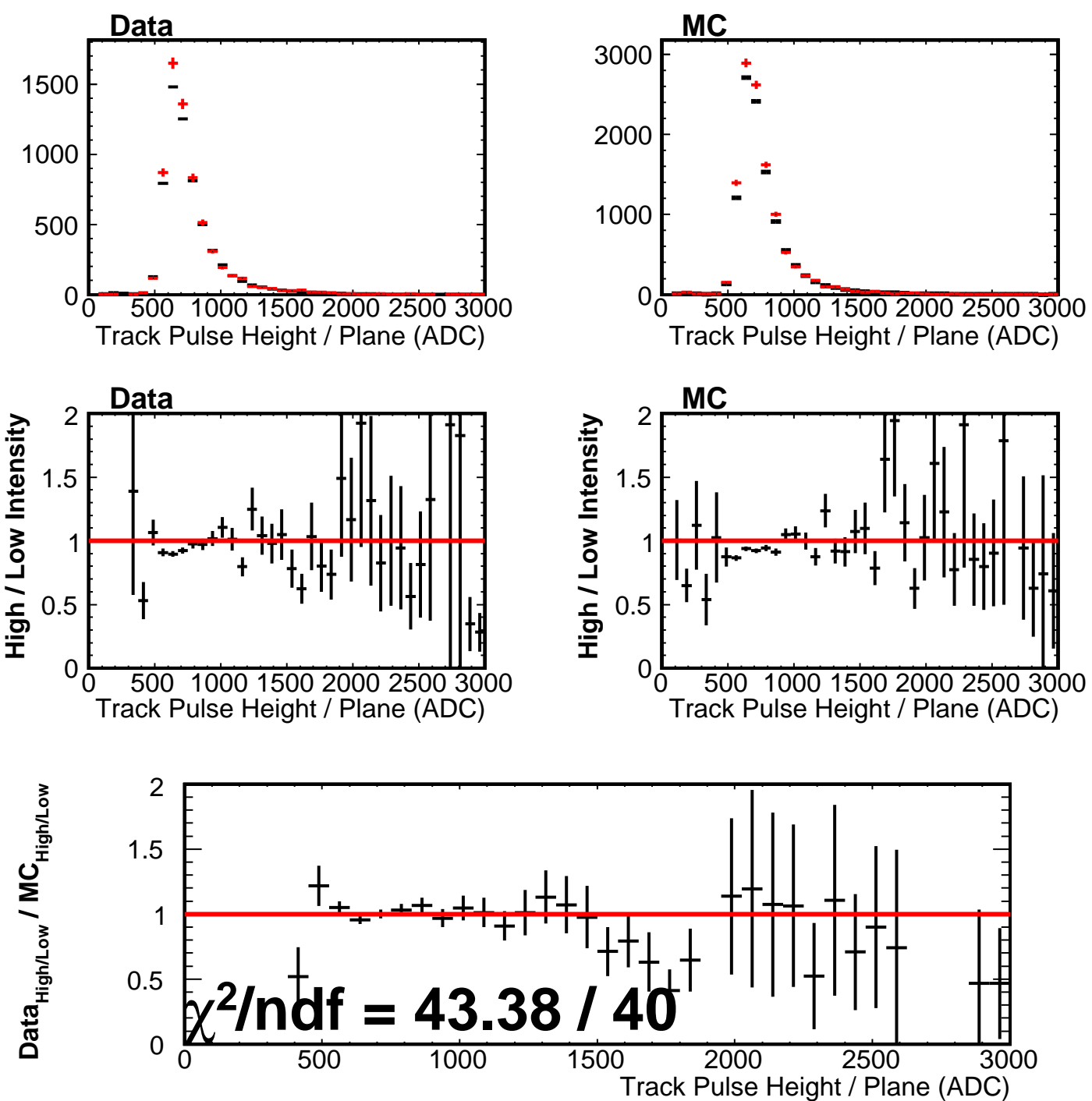

Figure 6.25: Comparison of the charged current track pulse height per plane for low and high intensities. 

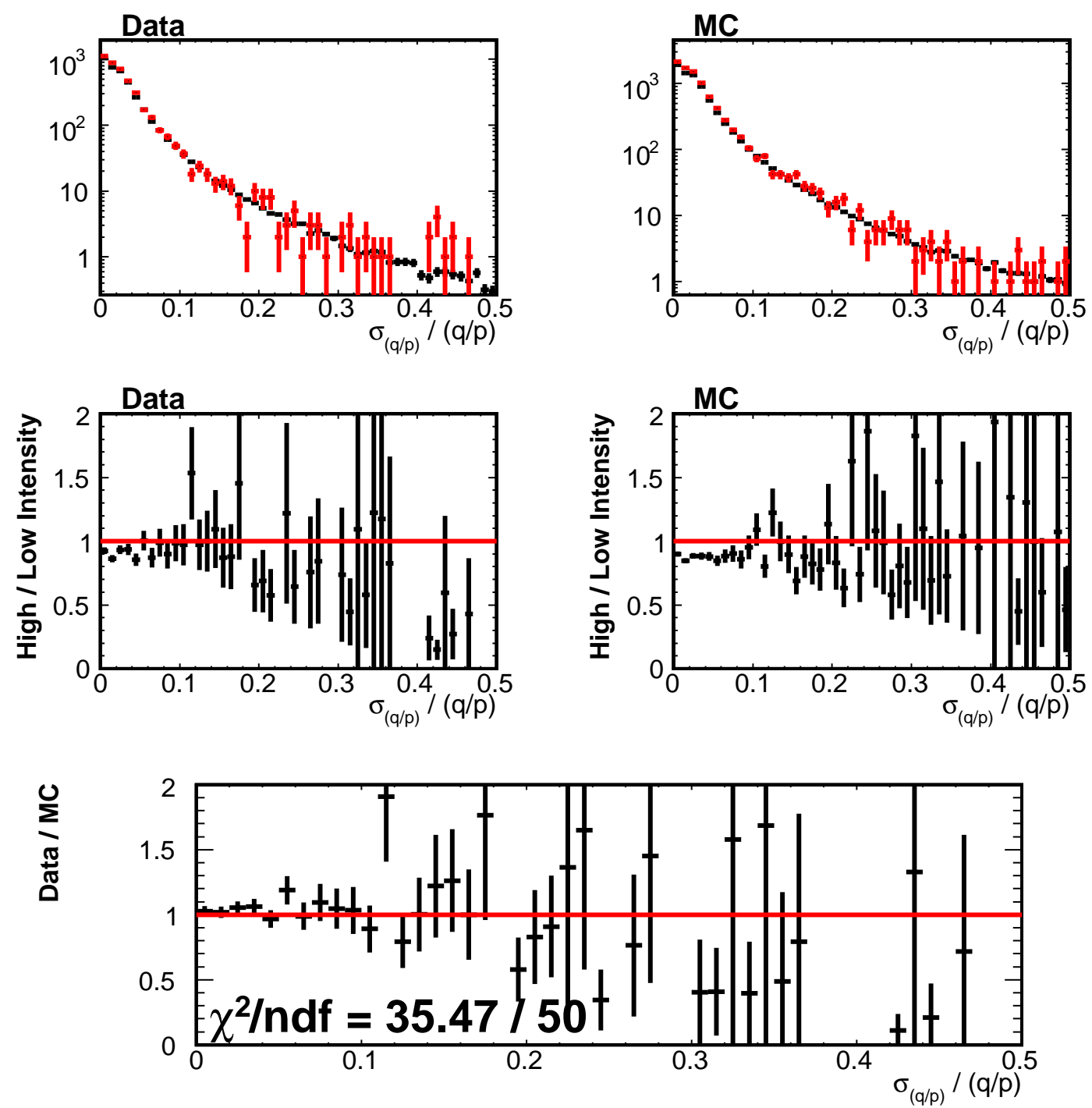

Figure 6.26: Comparison of $\sigma_{(q / p)} /(\mathrm{q} / \mathrm{p})$ for charged current tracks at low and high intensities. $\sigma_{(q / p)} /(\mathrm{q} / \mathrm{p})$ is defined as the fractional error on the quantity $\mathrm{q} / \mathrm{p}$ which is the charged divided by the momentum from curvature. 

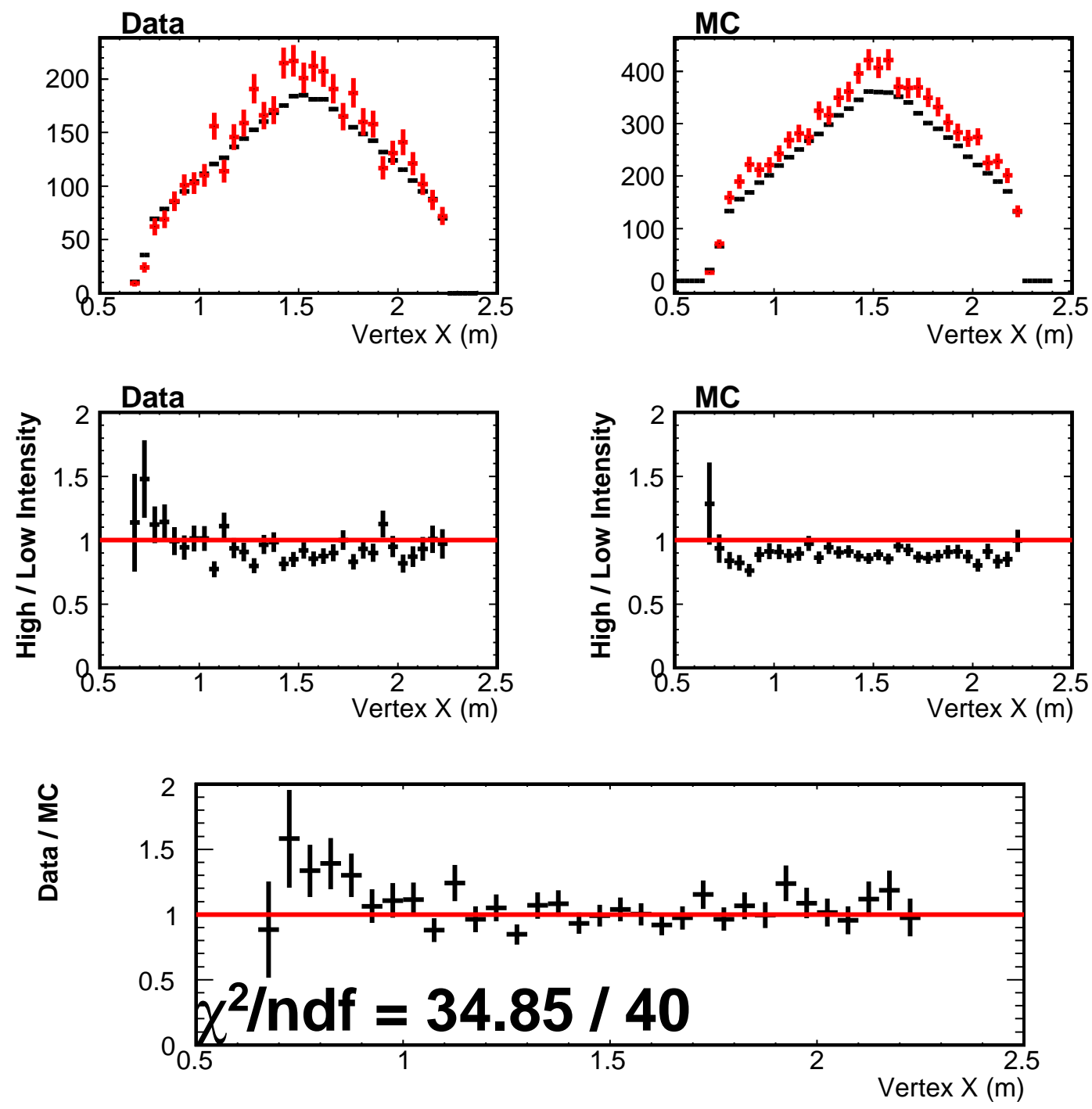

Figure 6.27: Reconstructed X vertex for all events. 

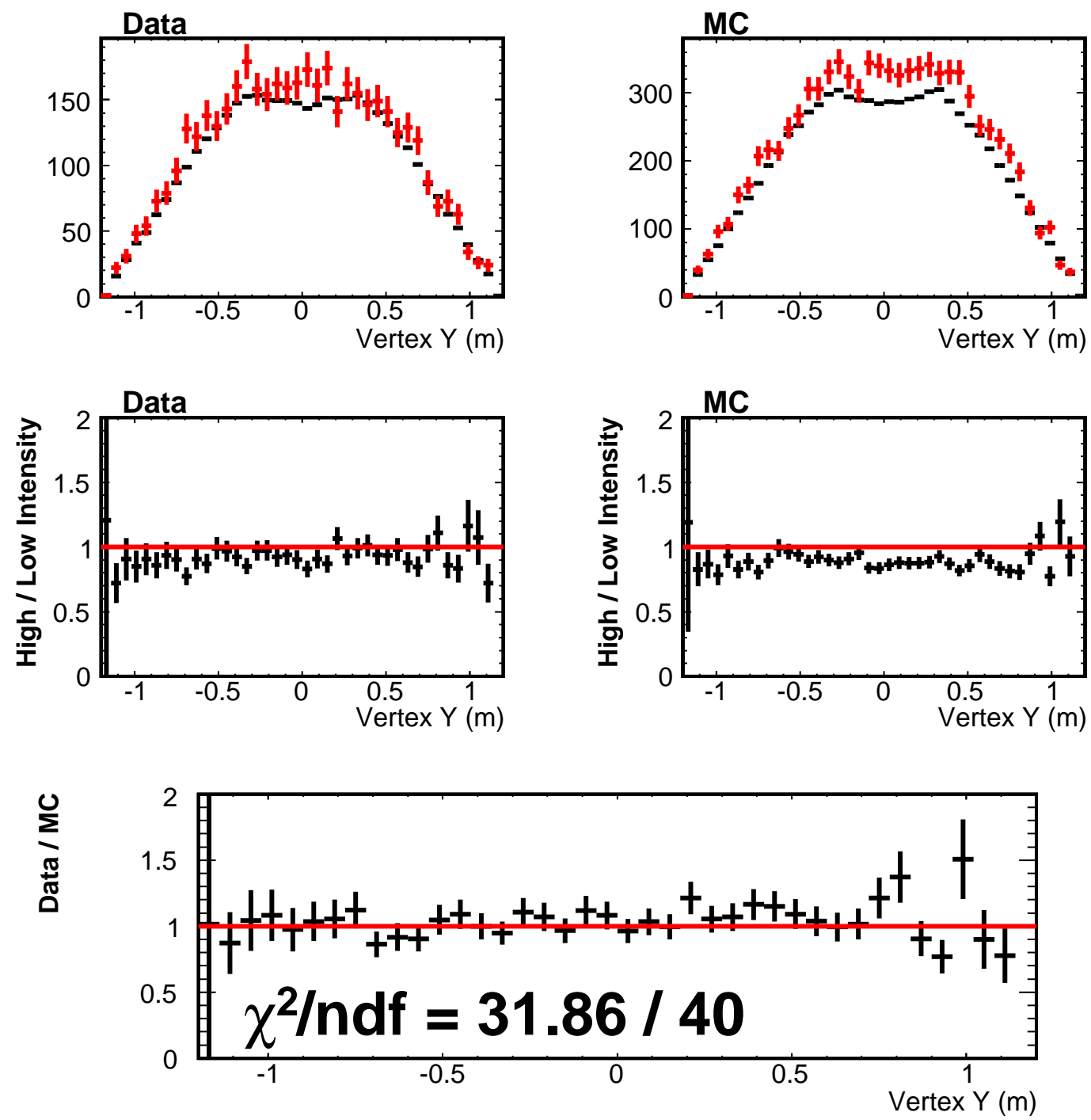

Figure 6.28: Reconstructed Y vertex for all events. 

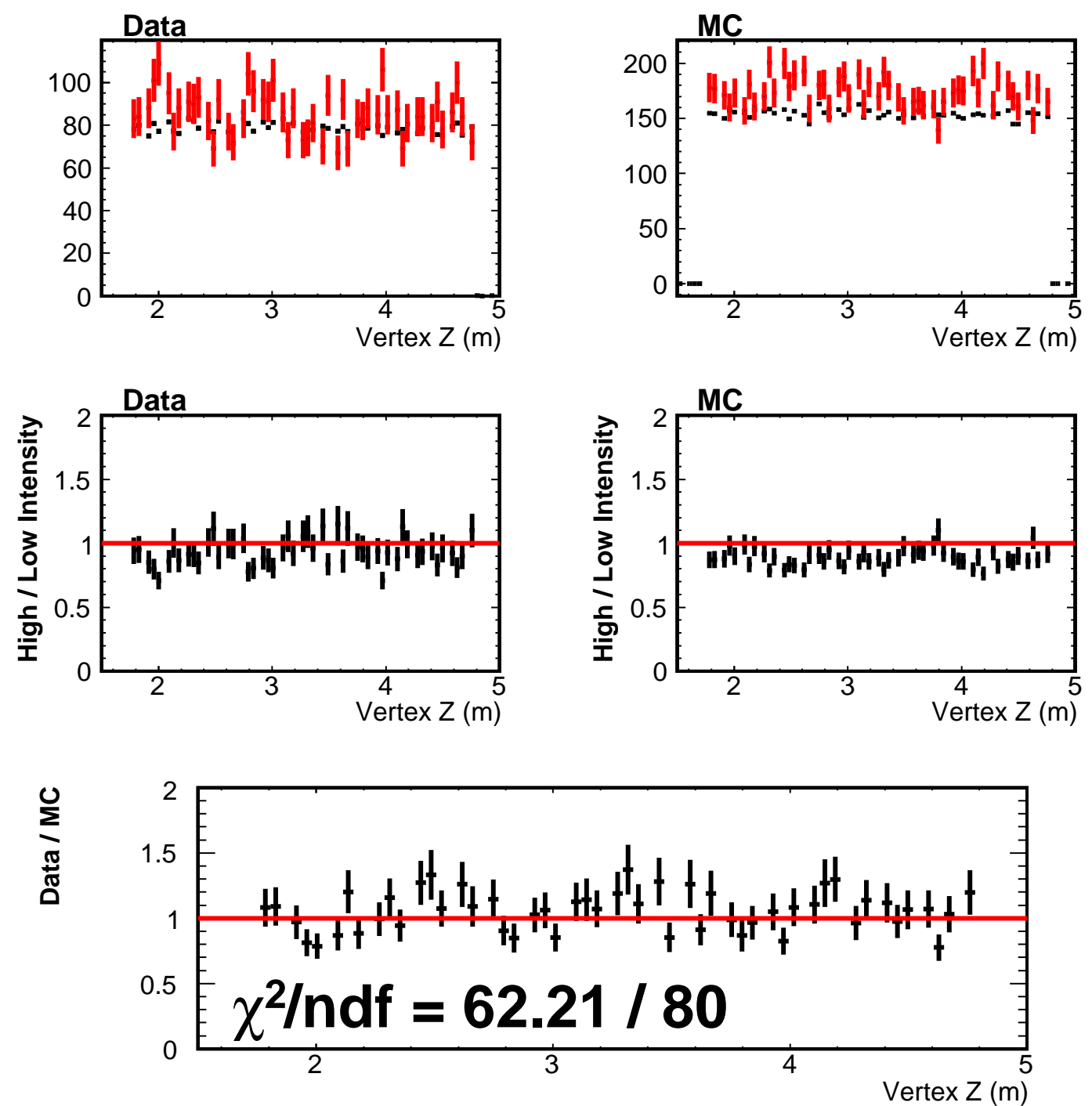

Figure 6.29: Reconstructed Z vertex for all events. 

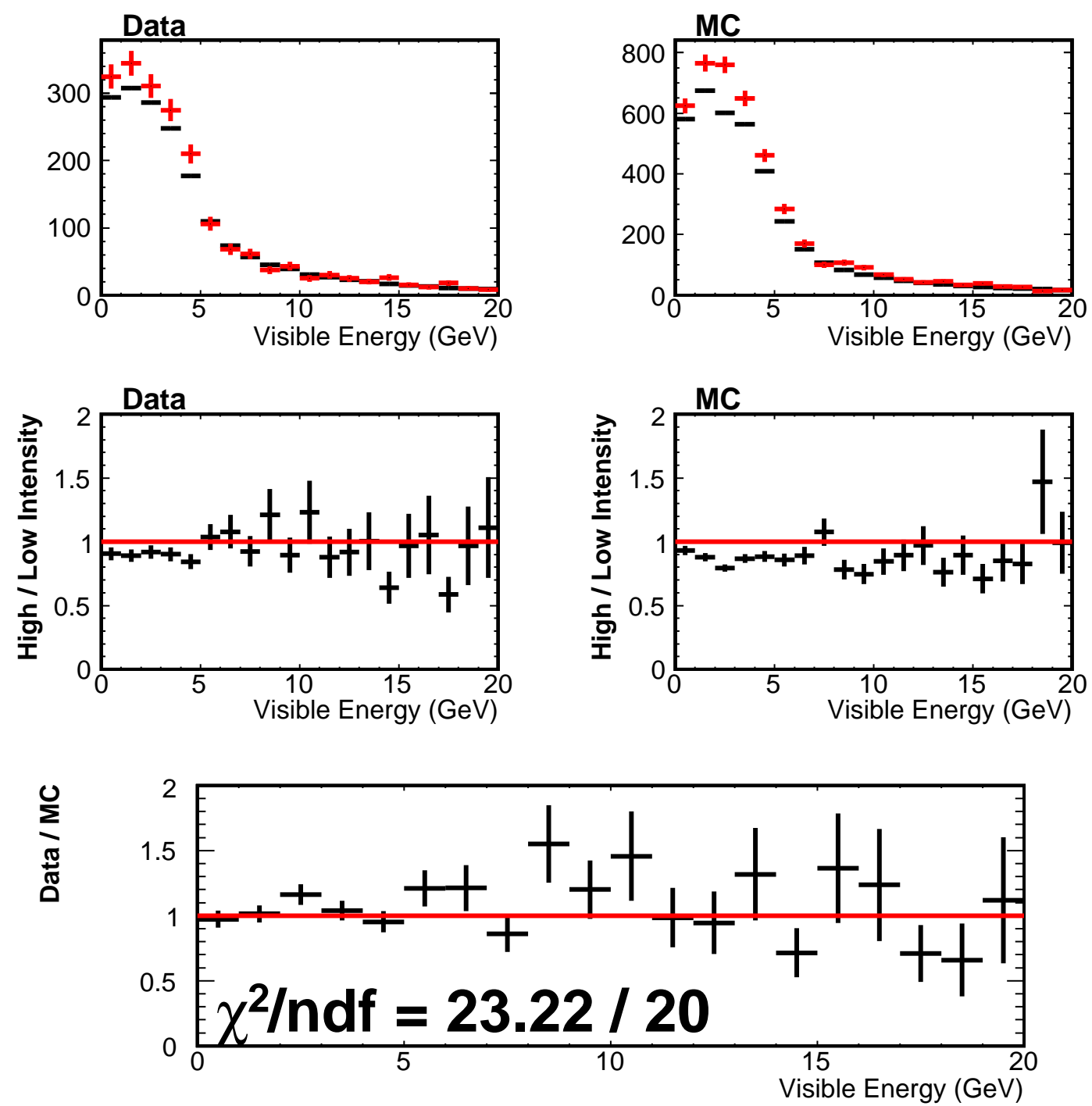

Figure 6.30: Comparison of the neutral current visible energy for low and high intensities. 

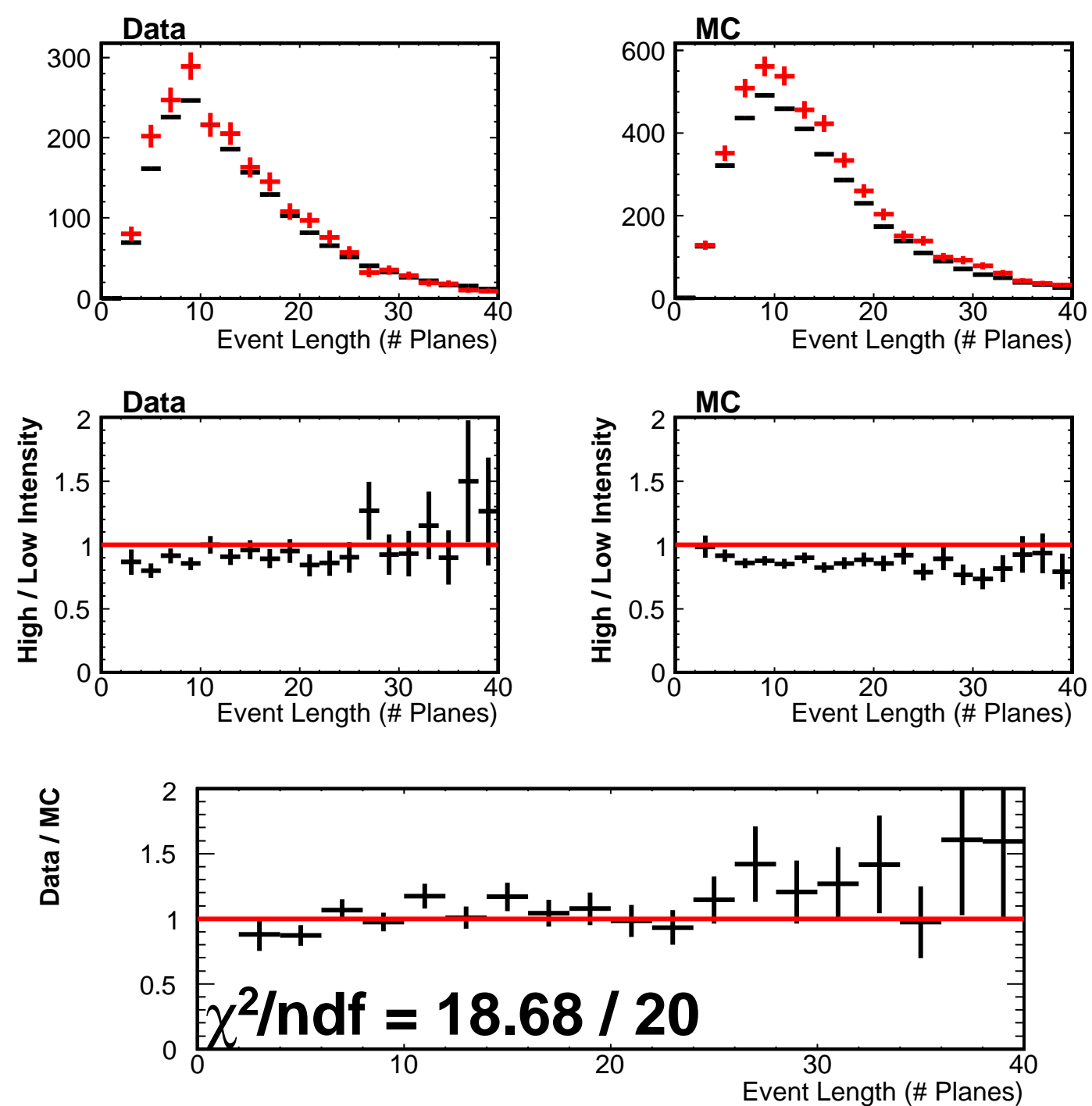

Figure 6.31: Comparison of the event length for neutral current events at low and high intensities. 

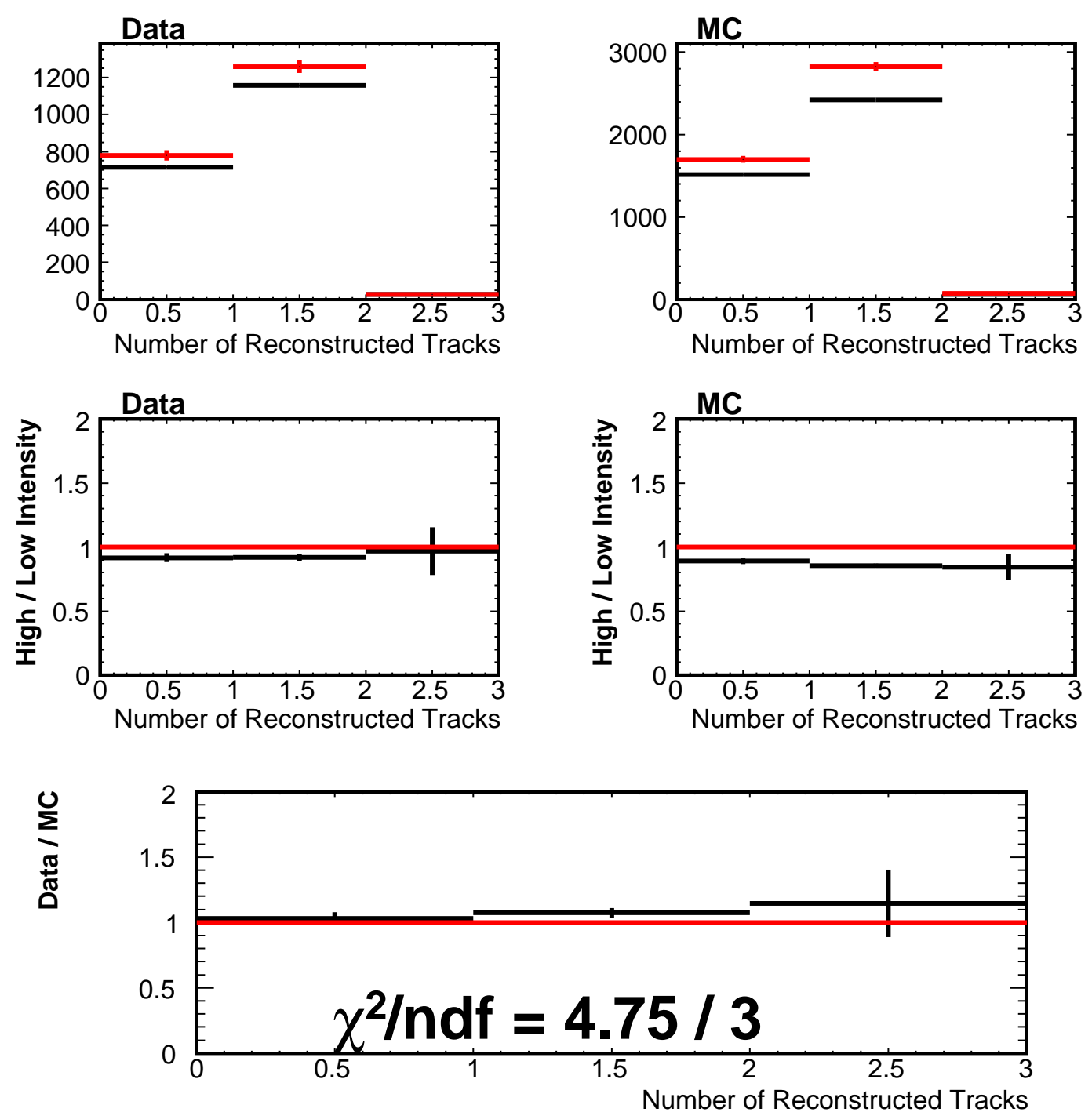

Figure 6.32: Comparison of the number of reconstructed tracks in neutral current events at low and high intensities. 

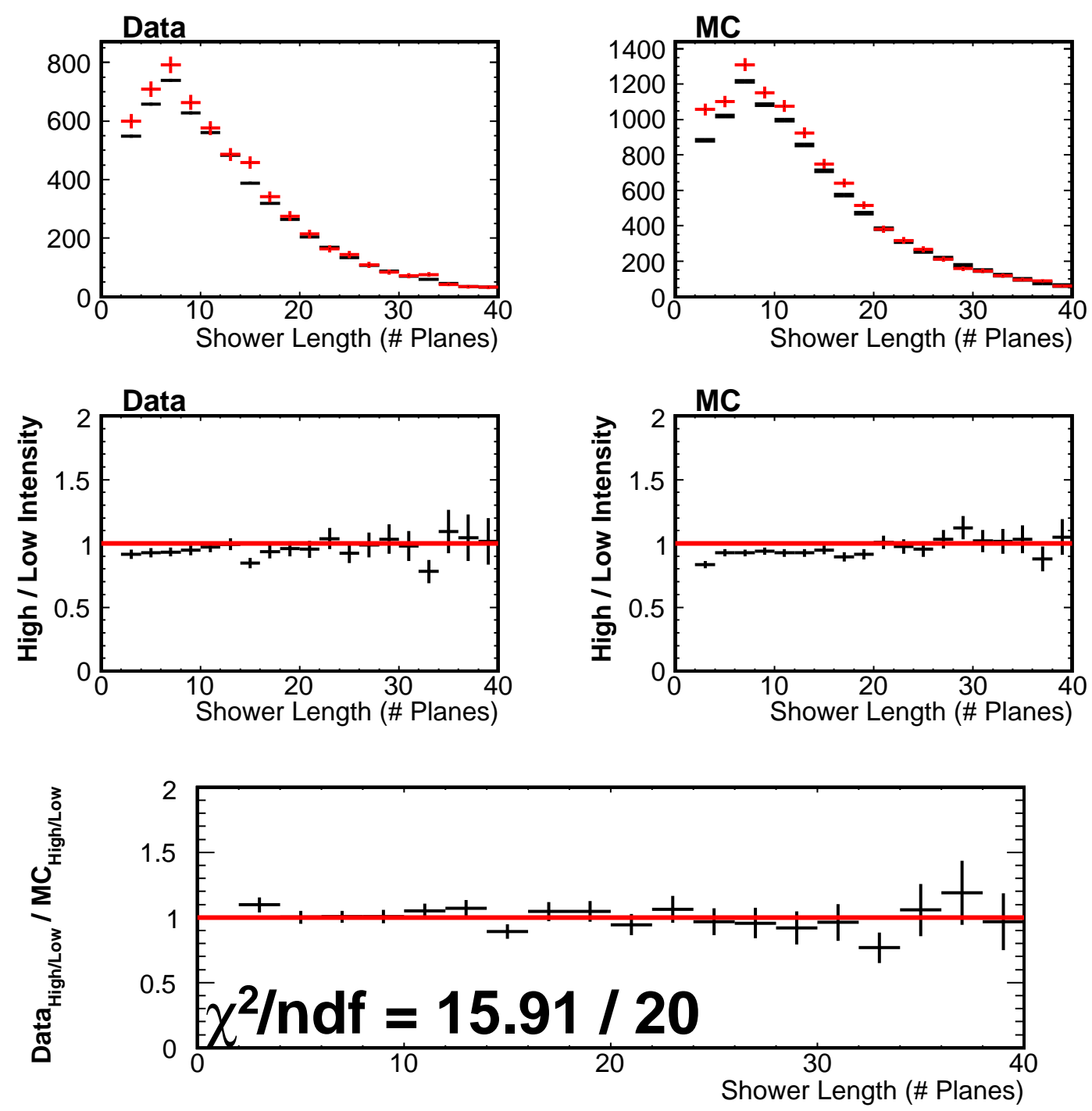

Figure 6.33: Comparison of the shower length for all showers at low and high intensities. 

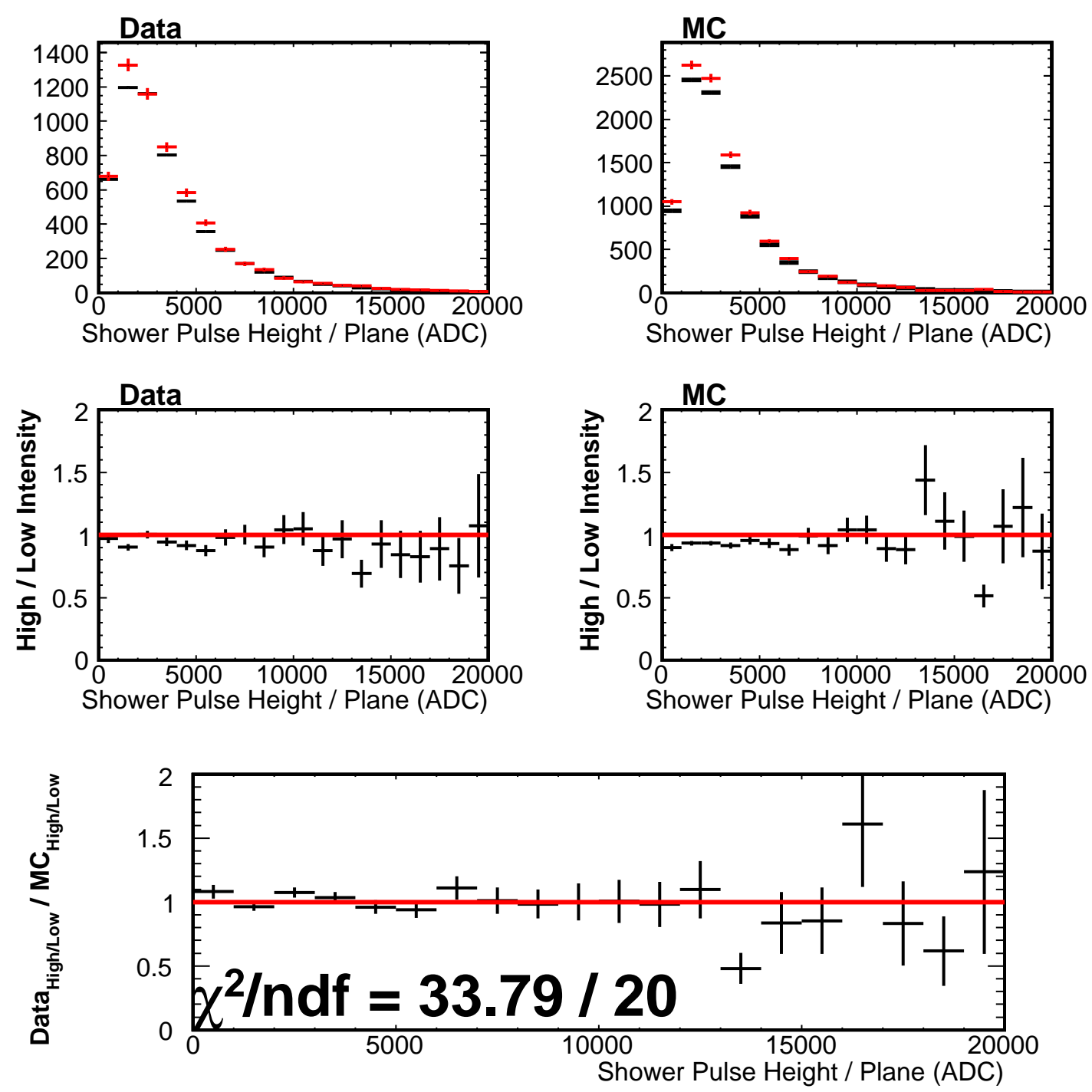

Figure 6.34: Comparison of the shower pulse height per plane for all showers at low and high intensities. 

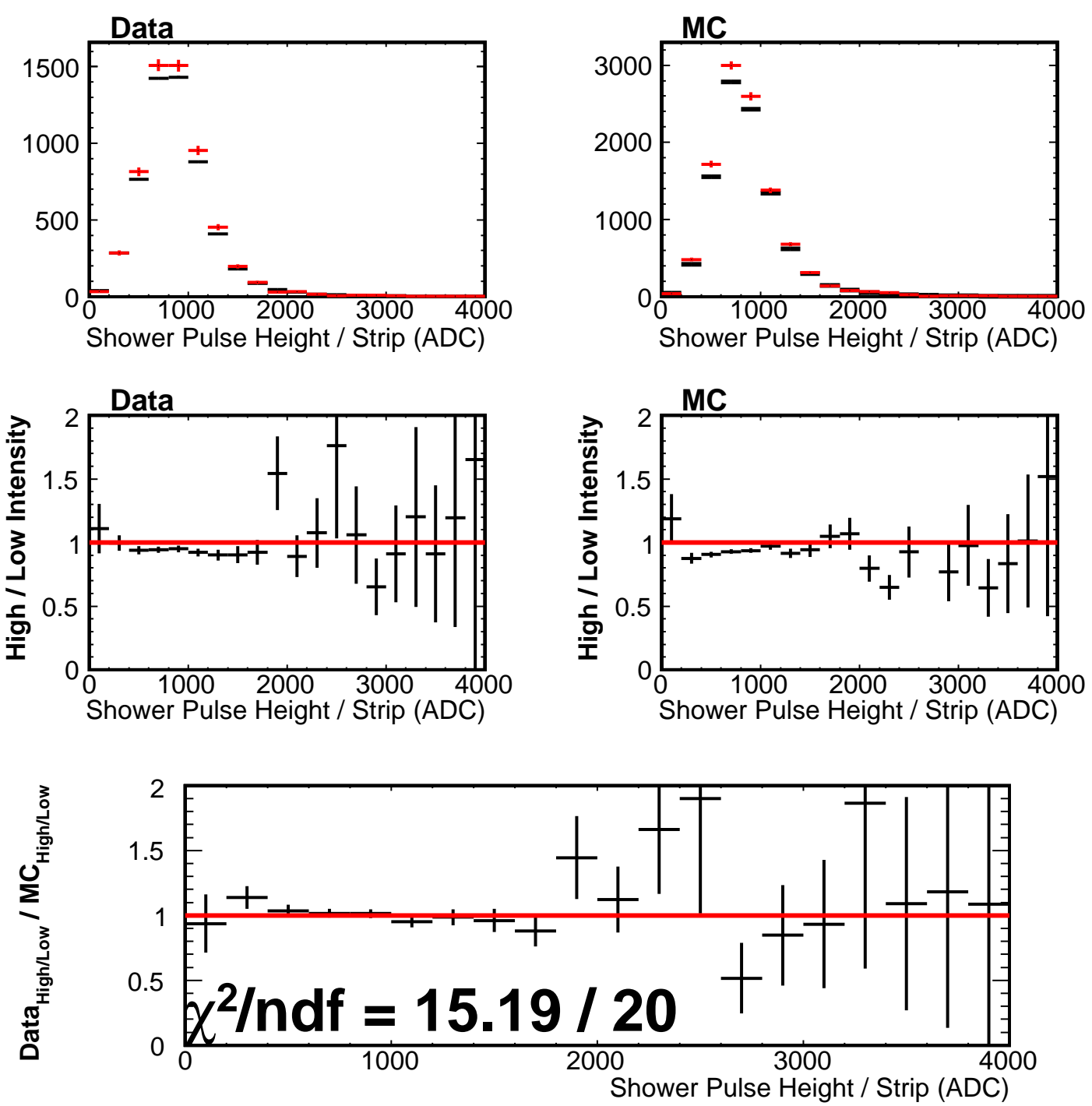

Figure 6.35: Comparison of the shower pulse height per strip for all showers at low and high intensities. 

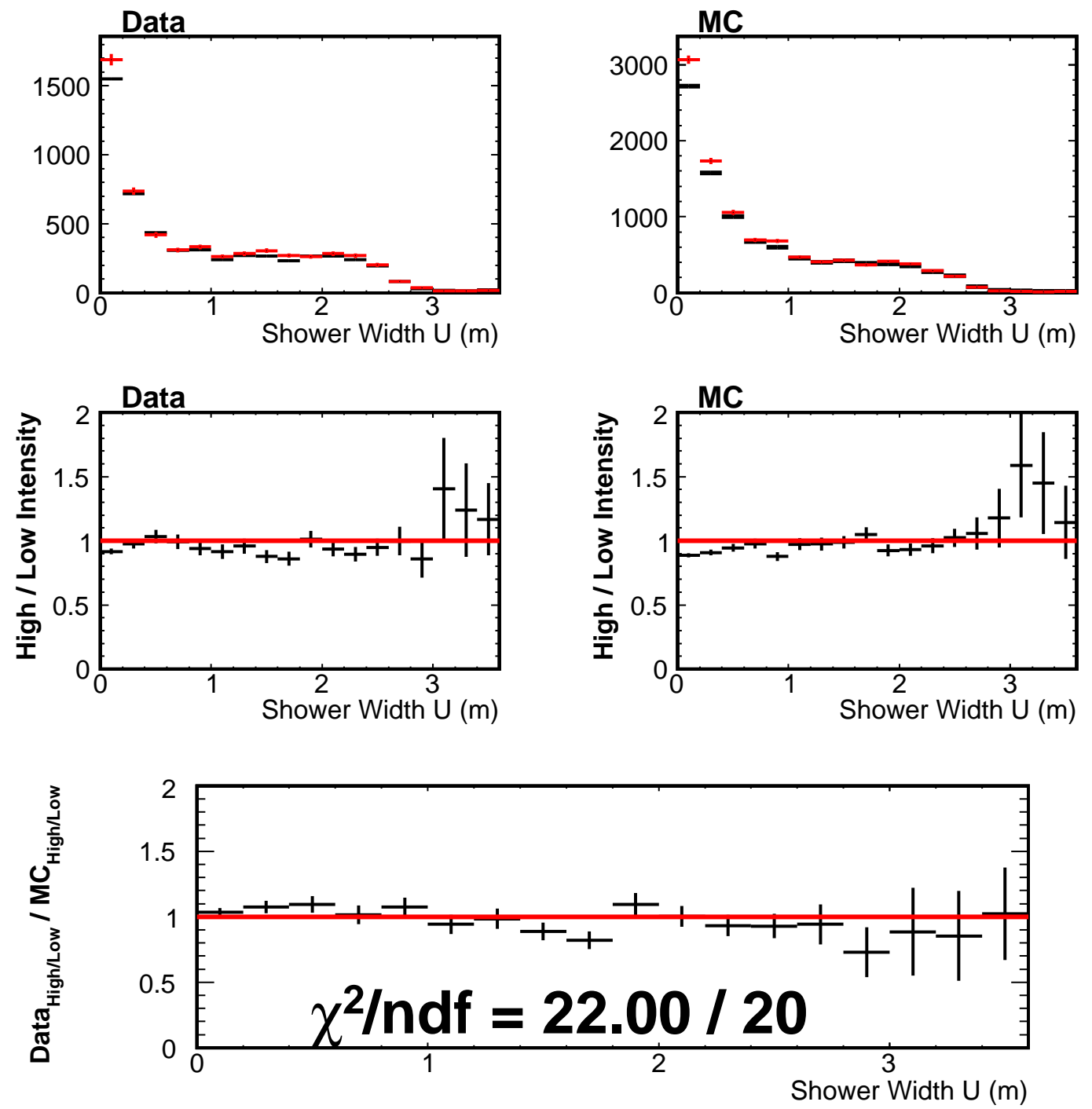

Figure 6.36: Comparison of the shower lateral width in the $U$ view for all showers at low and high intensities. 

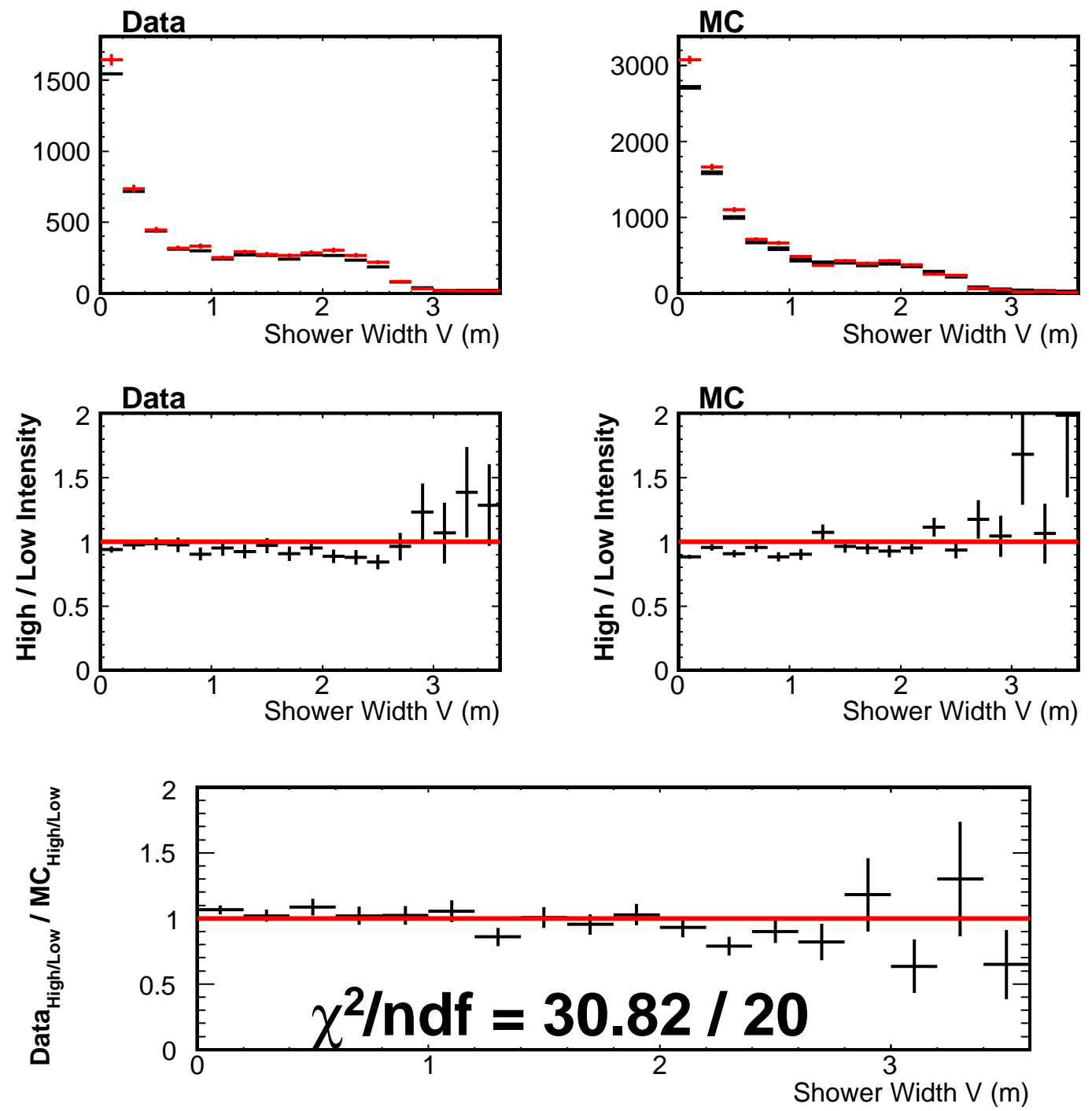

Figure 6.37: Comparison of the shower lateral width in the $\mathrm{V}$ view for all showers at low and high intensities. 


\subsection{Batch Studies}

Another interesting study is to examine various energy spectra as a function of NuMI proton batches. Figure 6.38 shows a picture of the Fermilab Booster in yellow and the Main Injector in red. The protons from the Booster can be injected to the Main Injector in a maximum of six proton batches. These batches are spaced roughly by 10 to $20 \mathrm{~ns}$. There are a few different modes that the Main Injector can run under. In NuMI-only mode, all six batches from the Booster are sent to the Main Injector for the NuMI beam line. In mixed-mode five batches are sent to the Main Injector, while the sixth is sent to produce anti-protons for the Fermilab Tevatron. The following plots use runs where the accelerator was in mixed-mode, and five batches are sent to NuMI.

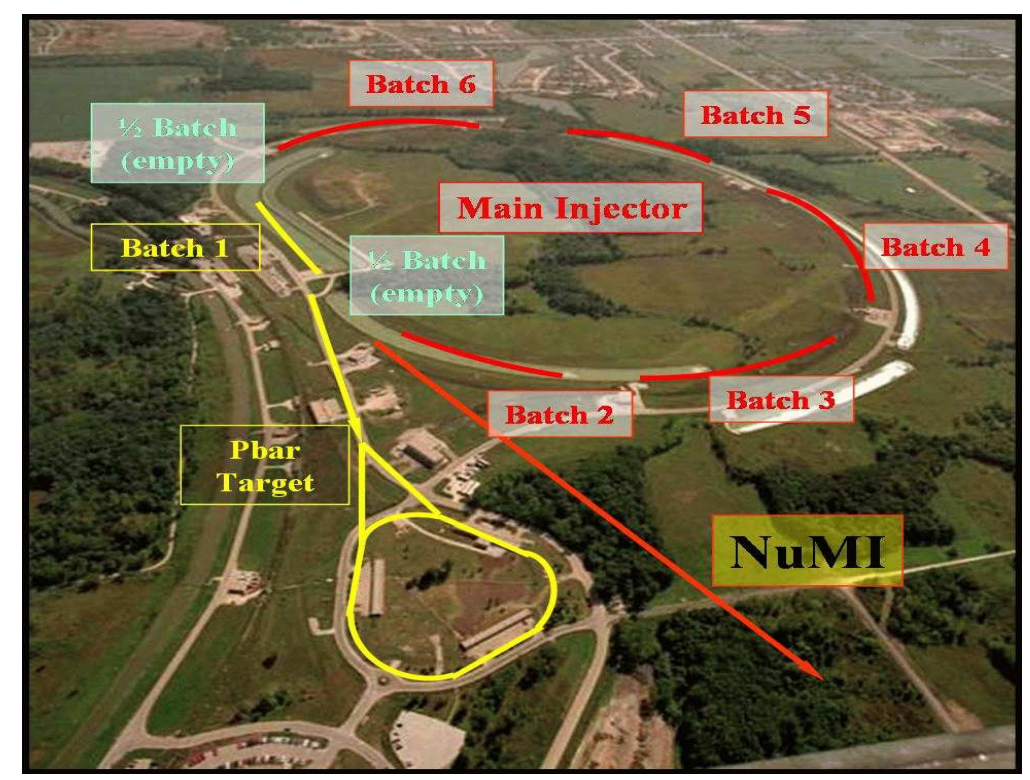

Figure 6.38: Aerial picture of the Fermilab Booster (yellow) and Main Injector (red) and labels indicating how different batches are distributed in the system [99].

Figures 6.39 through 6.41 we superimpose the charged current energy spectra, shower energy, and track momentum for five different batches. Let us take Figure 
6.39 as an example. The top plot shows five charged current energy spectra, where each color corresponds to a different batch as shown in the superimposed legend. The legend shows the five different batches, each with a different color, and the time of the strips in each batch on the $\mathrm{x}$-axis in nanoseconds. It is a testament to our reconstruction software and detector design that we can distinguish events in different batches. The following four plots show the ratio of the energy spectra from batch one to the other four. They are all centered at one, except for the ratio of batch one to five. This is because the fifth batch has less protons than the others due to accelerator issues, hence we do not expect the ratio to be at one. The two plots following this example have the same format and show equality between spectra amongst batches. 

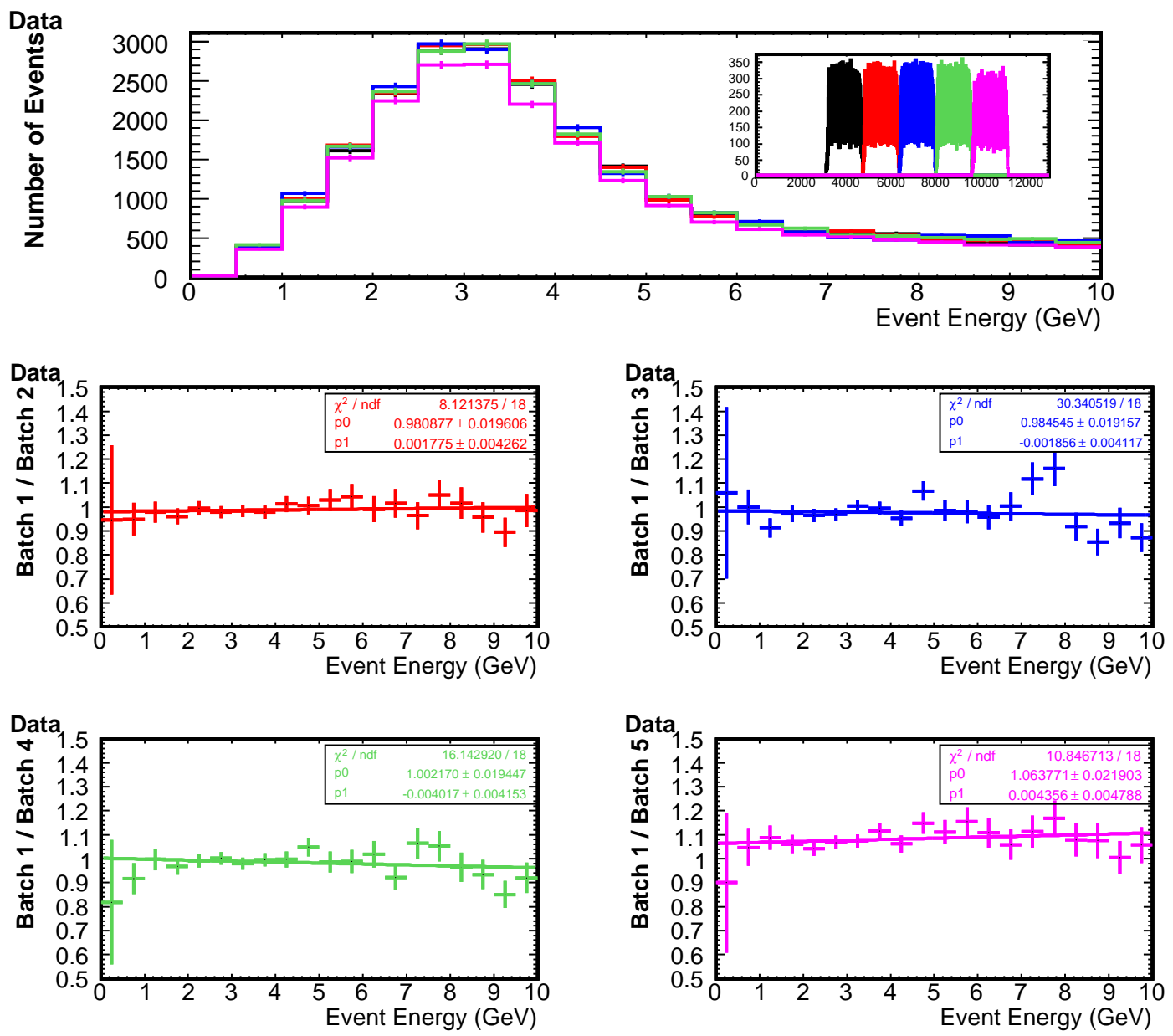

Figure 6.39: Charged current energy spectra from different NuMI batches. The top plot is the overlay of the different energy spectra for five different batches, given by the superimposed legend. The bottom four plots are the ratios of the spectra from the first batch to the others. Note the last batch has less protons on target than the other four so one does not necessarily expect a ratio of one. 

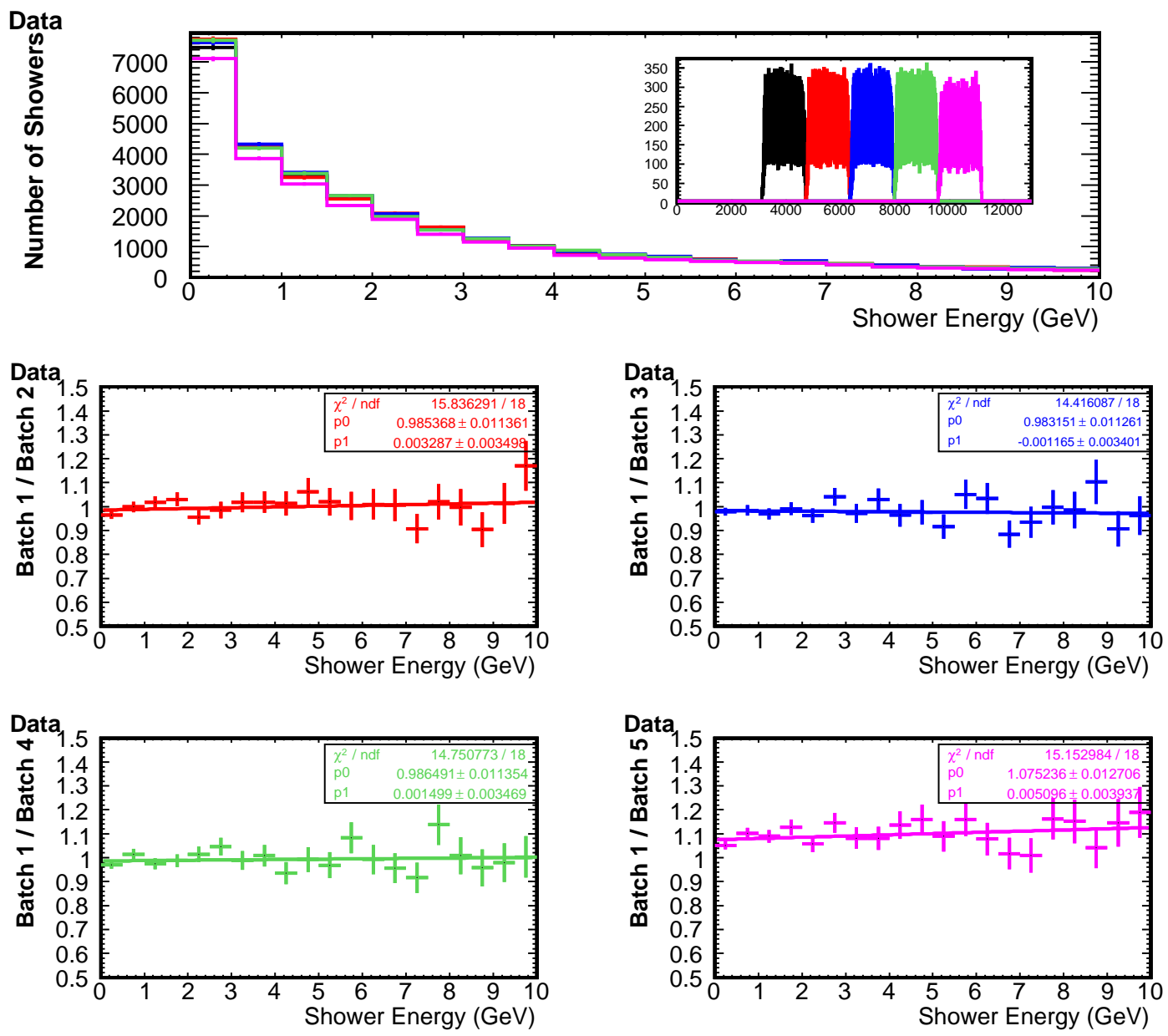

Figure 6.40: Charged current shower energy from different NuMI batches. The top plot is the overlay of the different energy spectra for five different batches, given by the superimposed legend. The bottom four plots are the ratios of the spectra from the first batch to the others. Note the last batch has less protons on target than the other four so one does not necessarily expect a ratio of one. 

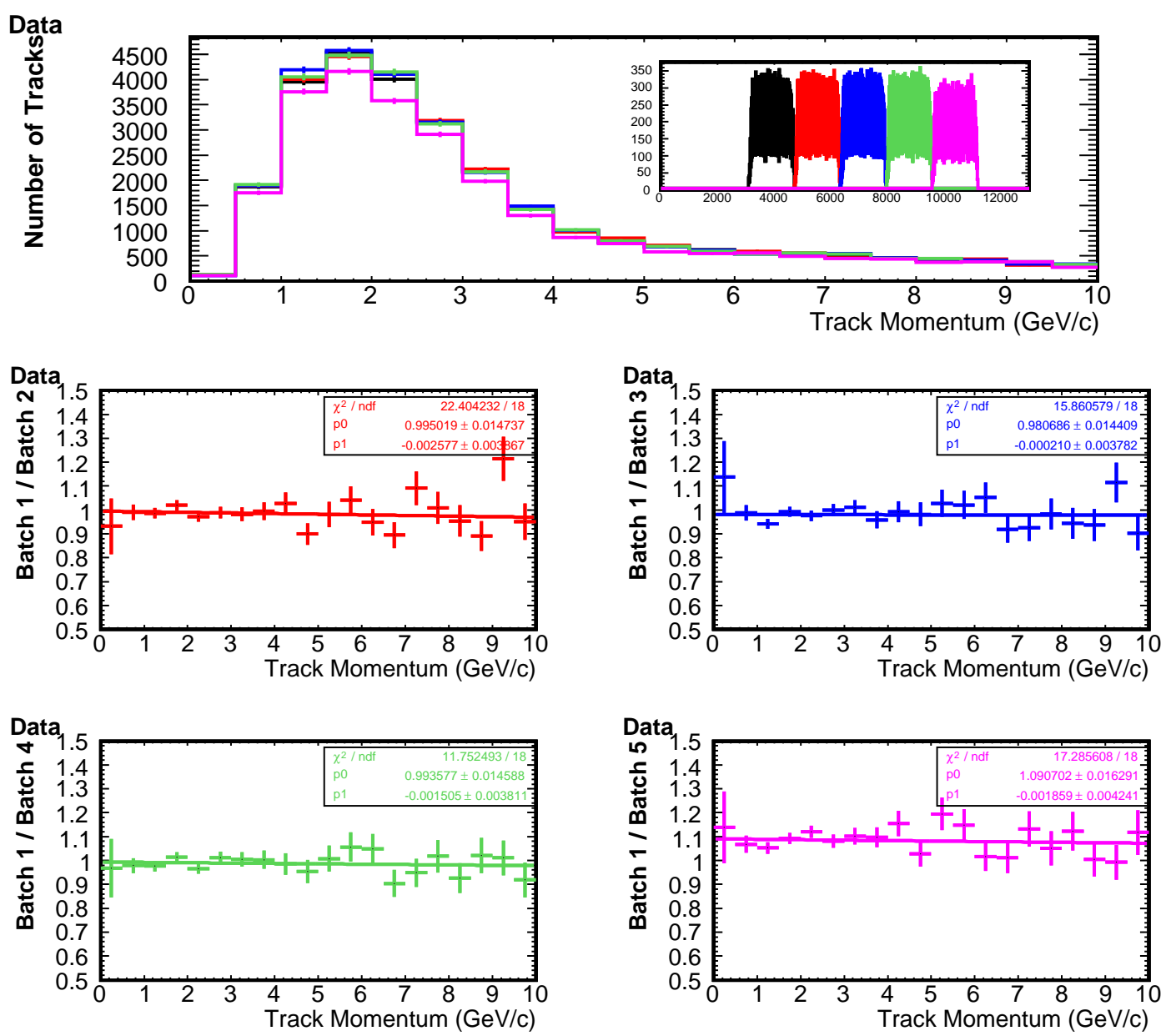

Figure 6.41: Charged current track energy from different NuMI batches. The top plot is the overlay of the different energy spectra for five different batches, given by the superimposed legend. The bottom four plots are the ratios of the spectra from the first batch to the others. Note the last batch has less protons on target than the other four so one does not necessarily expect a ratio of one. 


\subsection{Conclusions}

The conclusions drawn from this chapter are that our simulation properly models the Near detector and our analysis is not biased by intensity effects. There does exist a weak dependence on beam intensity that is modeled quite well by the Monte Carlo within the current statistics. Reconstruction is more efficient at shower and track finding at lower intensities and mainly at lower energies. Fewer interactions and hence a "cleaner" reconstruction environment at lower intensities allows our software to pick up smaller energy objects that would otherwise be lost at higher intensities.

The next few chapters begin our description of the oscillation analysis and measurement of $f_{\text {sterile }}$. We begin by discussing our fitting technique and the extrapolation of Near detector information to produce a Far detector prediction for the unoscillated spectra. 


\section{Chapter 7}

\section{NuMI Beam Neutrinos in the}

\section{Far Detector}

Event selection in the Far detector is very similar to the Near detector, described in Chapter 5, but there are additional criteria that must be applied in order to have a quality data set in which backgrounds are eliminated and neutrino events from the beam produced at Fermilab are properly selected.

When the Main Injector at Fermilab sends a pulse of protons to the target hall, a Global Positioning System (GPS) sends a signal to the Far detector indicating that a pulse of neutrinos is on its way. When the Far detector receives this signal it takes all data in a \pm 50 microsecond window that is centered on the prediction from the GPS at Fermilab. This is done to ensure that absolutely no neutrino interactions in the Far detector are lost. It is the data recorded in this time window that neutrinos will be selected.

This chapter outlines the procedure in which neutrino events are selected from the Fermilab beam and how the main sources of background are eliminated from the data. The actual neutrino events used in the oscillation analysis will be discussed in Chapter 9, along with the oscillation results. 


\subsection{Blinding Procedure}

In order to avoid bias in measuring oscillation parameters the MINOS collaboration has agreed to "blind" part of the data obtained in the Far detector [104]. This is known as a blind analysis and eliminates the conscious and unconscious biases that may affect physics analyses.

All Near detector data and Far detector data that is not within the $\pm 50 \mu \mathrm{s}$ time window can be analyzed without compromising the blinding procedure. This data is sufficient for studies in which the collaboration can be convinced that our data is understood and no pathologies exist that would affect our measurements.

Far detector data that occurs within the $\pm 50 \mu$ s time window will be split into two different streams, known as open and hidden. A sinusoidal function of total ADC counts and event length is defined and random numbers determine the frequency and phase of the function. Each data stream has approximately half of the total data. The open stream is available to all in the collaboration while the hidden stream is not analyzed until all steps of the analysis are complete.

\subsection{Main Sources of Background}

There exist three main sources of background in the Far detector. This includes events produced from the light injection calibration system, cosmic ray muons, and singles noise.

Light injection (LI) events occur when the light injection calibration system discussed in Chapter 3 happens to flash light during the $\pm 50 \mu$ s spill time window.

Cosmic rays muons enter the Far detector at a rate of about $0.5 \mathrm{~Hz}$. Since our time window for data taking lasts $100 \mu \mathrm{s}$, we should expect to see a cosmic event in one out of every 20000 beam spills, which is about the same rate as beam neutrino events. 
Singles noise can be categorized as noise in the electronics, dark noise originating in the photomultiplier tubes, or spontaneous photon emission from the wavelength shifting fibres. Since we apply the "2/36 trigger" in the Far detector, which requires at least two continuous planes with signal readout in a 36 plane window, this drastically reduces the amount of noise that enters the data stream.

The following sections describe criteria used not only to select beam neutrino events, but eliminate the backgrounds described above. Following these descriptions is a summary of the effects of these criteria on our Monte Carlo and the efficiency of removing backgrounds.

\subsection{Fiducial Volume Containment}

In order to accurately measure the energy of neutrino interactions, one has to ensure that the energy from these interactions is wholly deposited inside the detector. This is more of an issue for hadronic showers than for long $\mu$ tracks, since one can reconstruct their momentum using the magnetic field. A spatial set of fiducial volume cuts must be put in place to properly contain events. Since the Far detector has two supermodules that make up the detector, we define fiducial volume criteria that is analogous for both modules.

Longitudinally, a minimum depth of 4 scintillator planes $(0.2328 \mathrm{~m})$ is required from the front of a supermodule to veto events entering the front face of the detector. From the rear of each supermodule, a depth of $1 \mathrm{~m}$ is required to provide sufficient containment for the majority of showers [105]. Radially, we require that the event vertex be more than $50 \mathrm{~cm}$ away from the detector edge. This requirement is exactly the same as in the Near detector. We show the results of applying the fiducial volume to Monte Carlo in Figure 7.1.

A important by product of applying the fiducial volume cut is that it significantly eliminates some non-neutrino backgrounds to the analysis. The cosmic ray 
background is suppressed as the muons enter the edges of the detector. Fibre noise backgrounds are also heavily reduced because they typically tend to have very few strip hits and the demultiplexing algorithm tends to put these events at the edges of the detector.
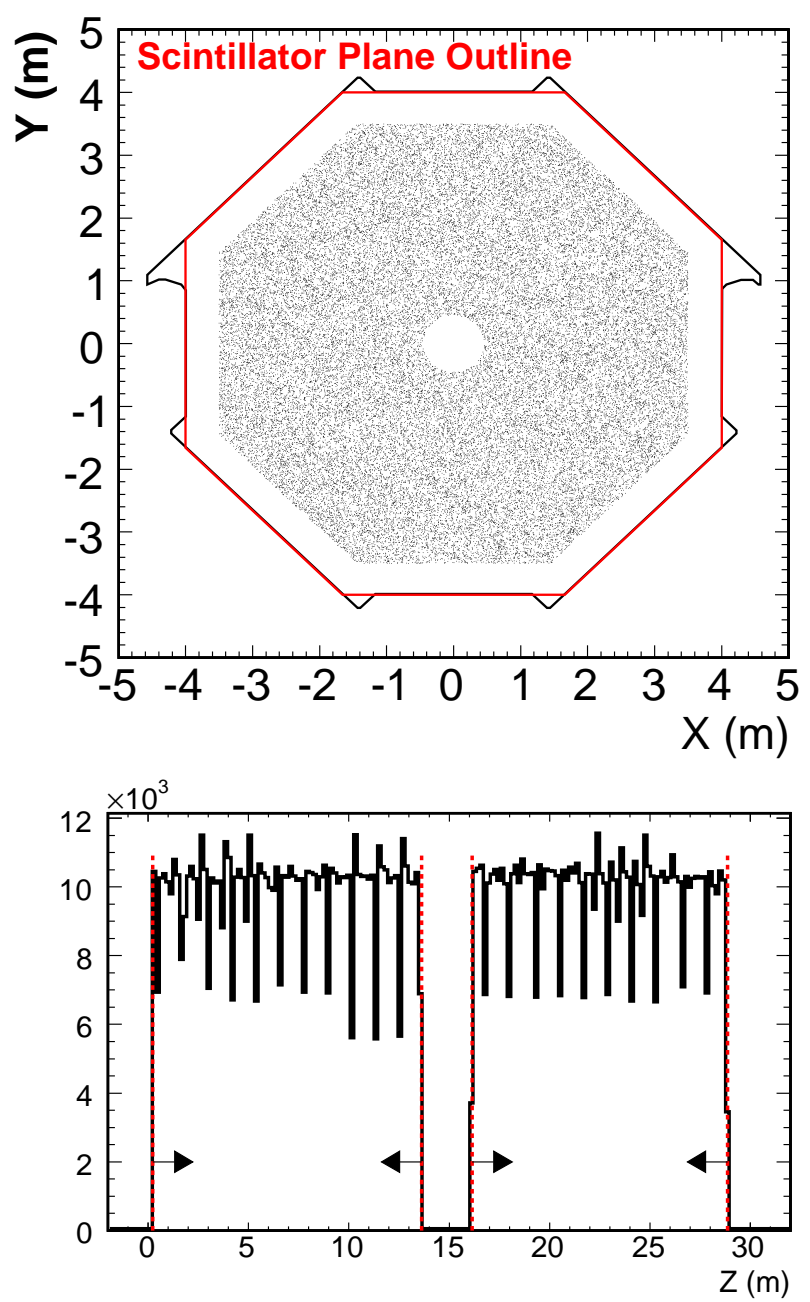

Figure 7.1: The effect of the fiducial volume requirement in the Far detector. The top plot is the radial fiducial volume, while the bottom plot is the longitudinal. 


\subsection{Data Cleaning}

\subsubsection{Removal of Light Injection Events}

Due to the low probability of getting a beam neutrino event coincident with an LI flash, we completely remove beam spills that contain these events.

These events are removed through the trigger photomultiplier tube (tPMT). The tPMT is illuminated directly by the LI system and hence is a very efficient (>99.99\%) way of identifying LI events. After this cut is applied a separate software package, called LISieve, is used to remove any other events that may be considered LI by using information from the light injection electronics to identify signals that are caused by the system itself.

We show in Figure 7.2 an example of a light injection event. The bottom left plot is the $\mathrm{U}$ view versus longitudinal position and the bottom right is the $\mathrm{V}$ view versus longitudinal position.
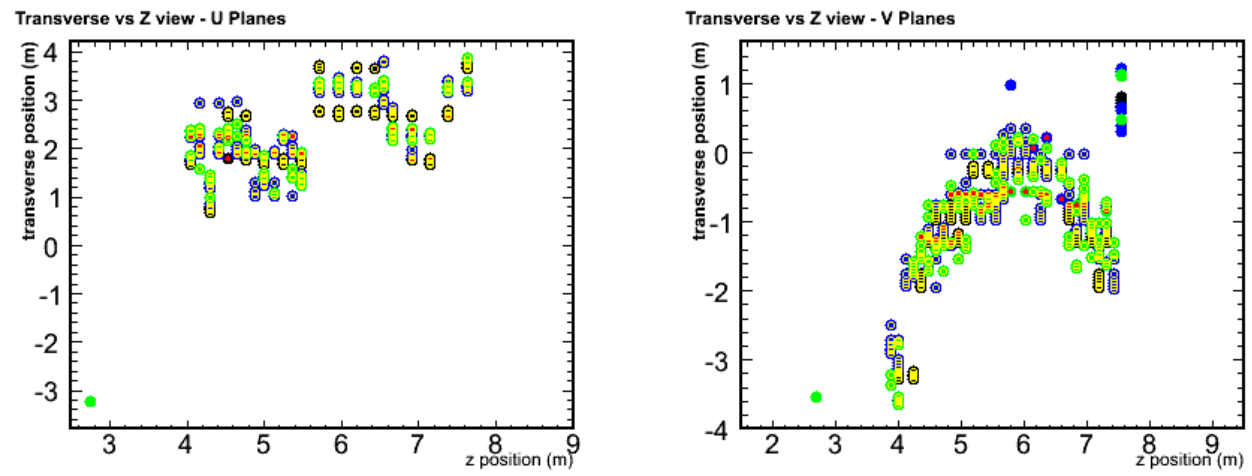

Figure 7.2: An example of a light injection event. 


\subsubsection{Singles Noise Removal}

In order for singles noise to be made into an event, the reconstruction software must match strip hits in both the $\mathrm{U}$ and $\mathrm{V}$ views, which implies that at least two noise hits are needed. Knowing this, we can conclude that random clusters of single fibre noise become exponentially less probable with larger numbers of strip hits, and hence a larger amount of energy deposited. To remove these types of noise, we remove events that either

- Have an energy pulse height of $<2500$ ADC counts and $\leq 8$ total strip hits.

- Have an energy pulse height between 2500 and 5000 ADC counts and $\leq 4$ total strip hits.

An example of a singles noise event is shown in Figure 7.3.
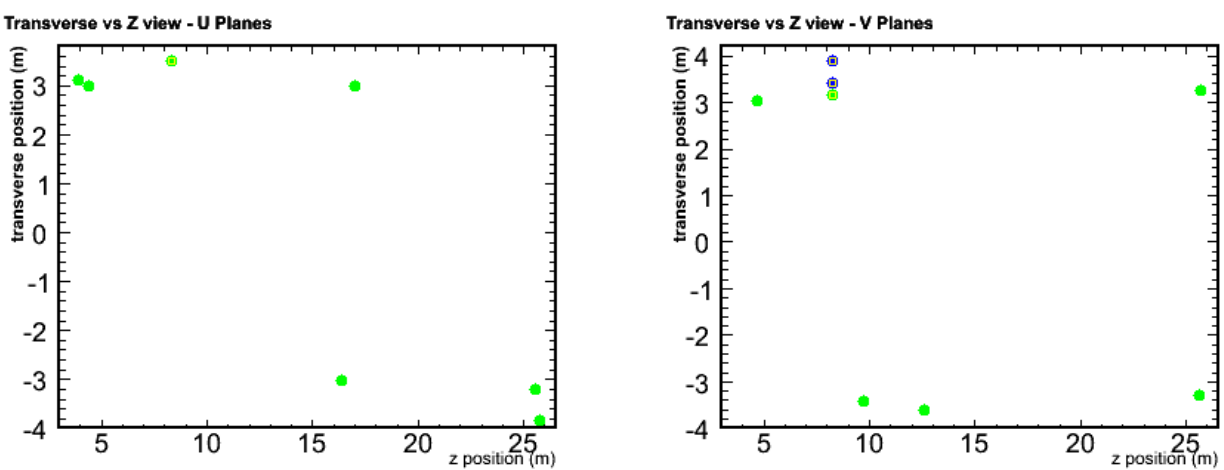

Figure 7.3: An example of a singles noise event. 


\subsubsection{Removal of Cosmic Ray Muons}

As described before, most cosmic rays are removed with our fiducial volume cut which removes events that enter through the edges of the detector. After this, the cosmics that remain tend to be less well reconstructed and are dependent on the angle the muon makes with the $\mathrm{z}$ axis. By making a cut on the absolute value of the $\mathrm{z}$ direction cosine, $\left|p_{z}\right| / \mathrm{E}>0.4$ we can accurately remove cosmic rays by taking into account their direction with respect to other beam neutrino events.

A second category of cosmics are those that are at high angles to the $\mathrm{z}$ axis. The tracking algorithm has trouble with these events, and are usually reconstructed as very steep showers and are not removed by the fiducial volume cut. These events tend to be very wide in comparison to their longitudinal length. We remove events that have a high ratio of transverse to longitudinal length. An example of a cosmic ray event is shown in Figure 7.4.
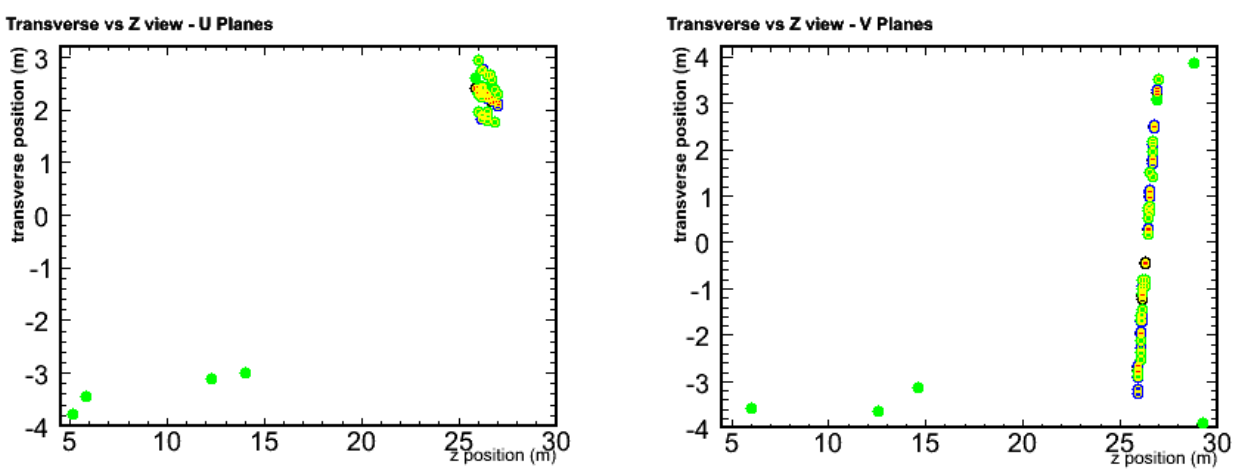

Figure 7.4: An example of a cosmic ray event. 


\subsubsection{Event Timing}

By only accepting events that occur in coincidence with the beam, the majority of backgrounds can be effectively eliminated. This is done by looking for a peak in the time difference between when an event is seen, and when the neutrino beam was thought to be passing through the detector. All beam neutrino events should be in this peak and we can discard events that lie outside of it. Since the beam spills from Fermilab last on the order of 10 microseconds, we can require a timing cut of $-2 \mu \mathrm{s}<t_{\text {time of event }}-t_{\text {time of spill }}<12 \mu \mathrm{s}$.

Figure 7.5 shows the distribution of the time difference between the event time and the start of the beam spill. The black histogram is all the spill events reconstructed in a months worth of data. The green component is the same distribution after the fiducial volume cut has been applied in the Far detector and the blue spectrum has the three data cleaning criteria applied. Notice that our data cleaning has left only events that satisfy the requirement of being in coincidence with a beam spill from Fermilab.

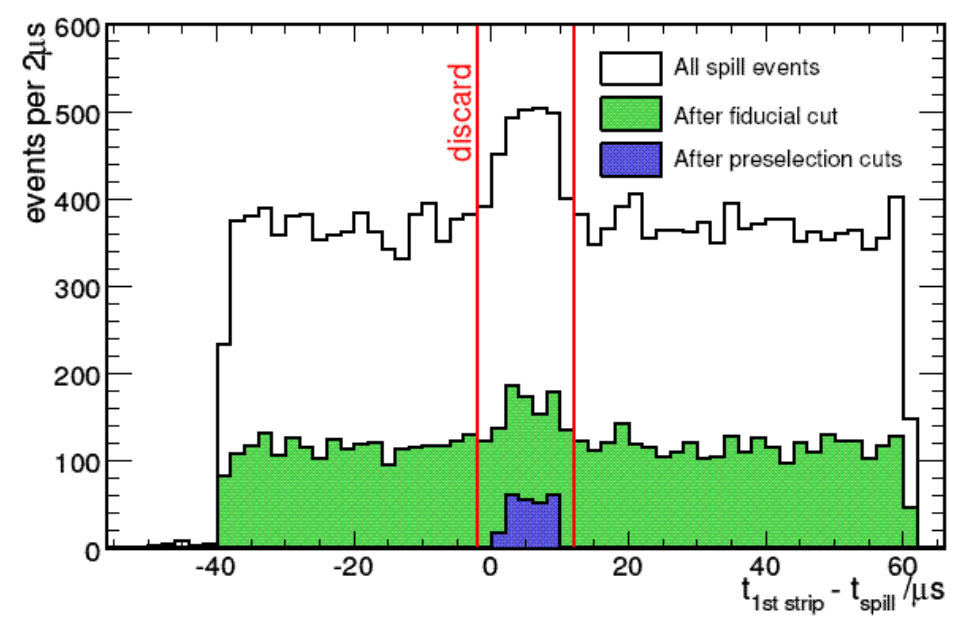

Figure 7.5: Time difference between events in the Far detector and the start time of the spill from Fermilab. 


\begin{tabular}{|c|c|c|}
\hline Data Cleaning Criteria & \# MC Events & Efficiency \\
\hline \hline LI Removal & 181473 & $100 \%$ \\
\hline Fiducial Volume & 124262 & $68.5 \%$ \\
\hline Noise & 122088 & $67.3 \%$ \\
\hline Cosmic Ray & 118215 & $65.1 \%$ \\
\hline
\end{tabular}

Table 7.1: The effect of data cleaning cuts on our Far detector Monte Carlo [105].

\subsection{Effects of Data Cleaning Criteria}

\subsubsection{Beam Neutrino Losses}

The data cleaning criteria defined to remove light injection, singles noise, and cosmic rays will remove some fraction of real beam neutrino interactions. Table 7.1 outlines the effect of these criteria on our Monte Carlo. The fiducial volume removes the most number of events, which is inevitable if we are to remove badly reconstructed events. Observe that only on the order of $3 \%$ of real events are removed with data cleaning while the next section will show that this is a small price for almost a complete removal of our backgrounds.

\subsubsection{Efficiency of Data Cleaning}

To estimate how many background events are left in the our data sample, we can use the events recorded by the fake spill-trigger. These are special runs that take data as if a neutrino spill was arriving from Fermilab in order to test how well we can remove backgrounds. Between May 2005 and February 2006, 1464005 of these fake spill triggers were recorded in addition to 3017209 beam spills that are in the open data stream, all of which have a time length of $100 \mu \mathrm{s}$.

Table 7.2 records the results of applying our final selection criteria and data cleaning defined in the previous sections to each of these spill sets. The first column labels the type of spill, with the fake spills first and where in time and out of 


\begin{tabular}{|c|c|c|c|c|}
\hline $\begin{array}{c}\text { Spill } \\
\text { Type }\end{array}$ & $\begin{array}{c}\text { Number of } \\
\text { Spills }\end{array}$ & $\begin{array}{c}\text { Window } \\
/ \mu \mathrm{s}\end{array}$ & $\begin{array}{c}\text { Exposure } \\
/ \mathrm{s}\end{array}$ & $\begin{array}{c}\text { \# Events } \\
\text { Passing }\end{array}$ \\
\hline Fake spill & 1464005 & 100 & 146.4 & 2 \\
\hline Spill (out of time) & 3017209 & 86 & 259.5 & 0 \\
\hline Spill (in time) & 3017209 & 14 & 42.2 & 246 \\
\hline Estimated background & & & & 0.208 \\
\hline
\end{tabular}

Table 7.2: Summary of the effect of event selection and data cleaning on fake trigger spills and in and out of time beam spills. [105]

time spills refer to the $-2 \mu \mathrm{s}<t_{\text {time of event }}-t_{\text {time of spill }}<12 \mu$ s event timing requirement and all other events outside of this timing cut for beam spills. The second column records the total number of spills, the third column is the length of the time window for each spill condition. The fourth column records the number of spills per second and the fifth column is the total number of events passing all the selection and data cleaning criteria. The in time beam spill shows 246 events, of which all should be neutrino events. Any event passing the other two spill categories must be backgrounds since they are not in coincidence with the Fermilab beam.

The total exposure per second for the fake spills and out of time spills is 405.9 spills per second, compared with a total exposure of 42.2 spills per second for the in time spills. If only two background events appear in the fake and out of time spills then we expect $2 \times 42.2 / 405.9=0.208$ background events in our open data sample of 246 events. This corresponds to an expectation of $<0.1 \%$ contamination of background events in our final data sample. We can be assured that our event selection and data cleaning are indeed finding true beam neutrinos and rejecting backgrounds with a high efficiency. 


\subsection{Energy Spectra in the Far Detector}

Charged current and neutral current energy spectra are selected in the Far detector exactly as we defined for the Near detector in Chapter 5. In addition to this, the neutrino flux tuning is applied to our Far detector Monte Carlo as well. We show these distributions for Monte Carlo with and without the flux tuning applied for charged current and neutral current energy spectra in Figure 7.6.
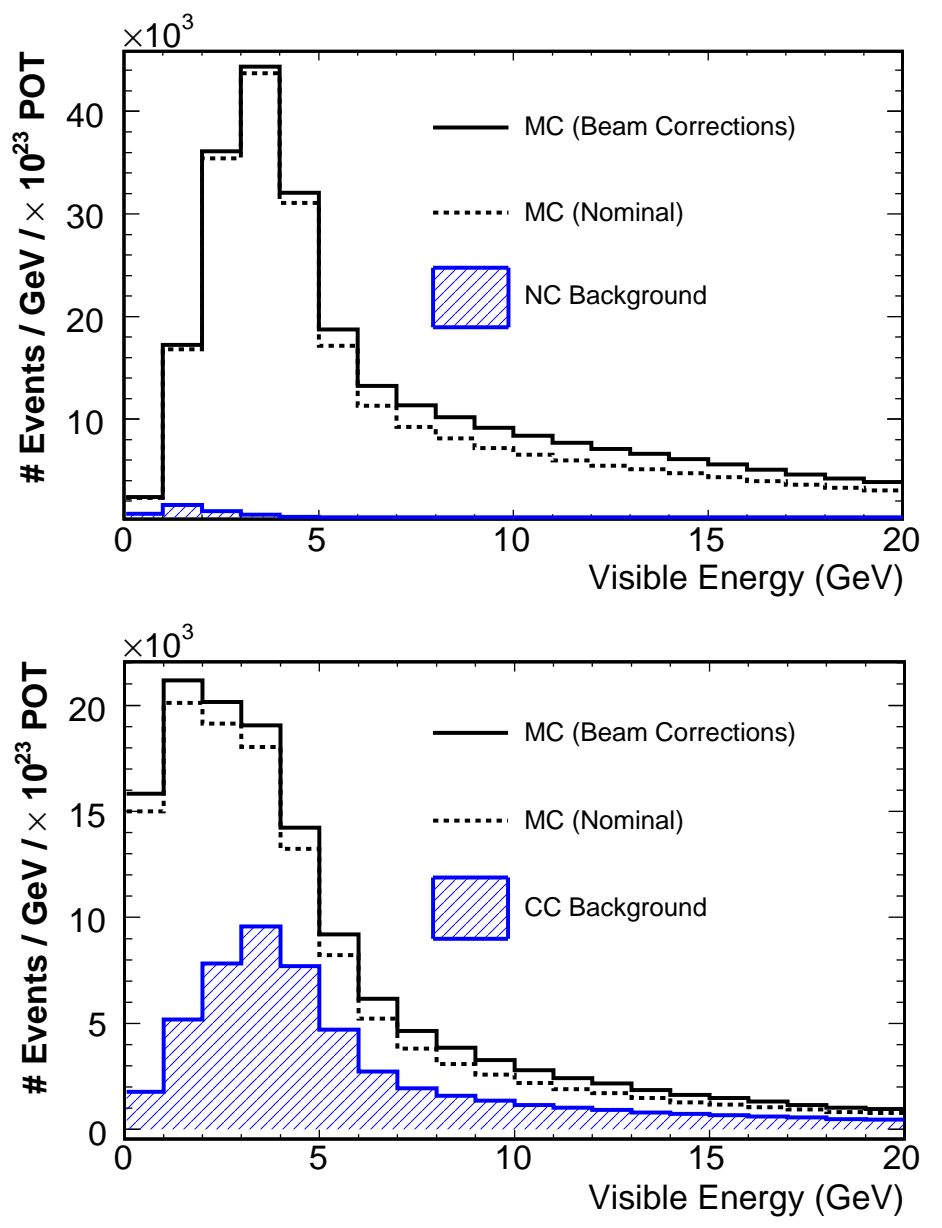

Figure 7.6: The top plot is the charged current and bottom is the neutral current selected energy spectra for the Far detector Monte Carlo. 


\section{7 $\quad$ Summary}

A method has been described to properly select neutrino produced from the beam at Fermilab as well as a procedure for reducing nearly all our backgrounds in the Far detector. The results of this chapter give confidence to our ability to construct a quality data set in the Far detector. The next chapter outlines the analysis method that will be used in the oscillation measurement. Discussed is how one uses the Near detector data to resolve differences between data and Monte Carlo followed by how to use this information to accurately measure oscillation parameters in the Far detector. 


\section{Chapter 8}

\section{Extrapolation from the Near to}

\section{Far Detector}

Methods to select charged current and neutral current events in the Near and Far detectors have been developed, in addition to performing several studies that show no biases in the Near detector. Now that events can be selected in order to do the oscillation measurement, we proceed with describing the analysis chain. This chapter begins by describing the sequence of using the Near detector data to make a prediction for the Far detector energy spectra in the absence of oscillations, otherwise called the extrapolation method. A brief commentary on the minimization technique used in this analysis follows. After developing the analysis method, systematic studies are discussed, in which different systematic errors are introduced to see how our knowledge of certain physical parameters will affect the oscillation measurement. Last, the results of the "Mock Data Challenge" are described, in which two independent data sets with unknown oscillation parameters applied were produced for individuals to test whether their fitting routines can extract the correct input oscillation parameters.

In Chapter 5 It was commented that there do exist disagreements between 
data and Monte Carlo for the Near detector CC-like and NC-like energy spectra as seen in Figure 5.10. If these differences are not accounted for it is expected that these disagreements will remain in the Far detector and hence bias our oscillation measurement. In order to improve agreement between the observed energy distributions and those predicted by the Monte Carlo, a simultaneous fit is performed to the measured Near detector charged current and neutral current energy spectra by varying a certain set of systematic parameters to deal with cross-section and reconstruction systematics. These parameters will be discussed later. The minimization scheme used here is called Marquardt fitting [107]. A brief outline of the method is as follows:

1. The Near detector data is fit for a certain set of systematic parameters to resolve differences between the data and Monte Carlo.

2. The best fit parameters are applied from the Near detector fit to the Far detector Monte Carlo in order to gain a prediction in the absence of oscillations

3. A oscillation fit is performed for $\Delta m^{2}, \sin ^{2}(2 \theta)$, and $f_{\text {sterile }}$ in the Far detector.

The method is called the Marquardt-style NDFit method. The "NDFit" refers to the extrapolation method in which the Near detector is fit for a certain set of systematic parameters and then the best fit parameters are applied to the Far detector Monte Carlo in order to do the oscillation analysis. The NDFit has been previously developed [103] for the MINOS charged current results, but did not utilize Marquardt fitting, which is why the method is labelled Marquardt-style. The Marquardt-style minimization is discussed in the next section.

\subsection{Marquardt-Style Minimization}

The Marquardt fitting [107] method first fits for a number of systematic parameters in the Near detector by minimizing the following statistic: 


$$
\chi^{2}=2 \times\left(\sum_{i}^{n} N_{i}^{e x p}-N_{i}^{o b s}+N^{o b s} \ln \frac{N_{i}^{o b s}}{N_{i}^{e x p}}\right)+\sum_{j}^{m} \frac{\epsilon_{j}^{2}}{\sigma_{j}^{2}}
$$

The first sum takes into account the Poisson nature of the distributed data in the Near and Far Detectors. $N_{i}^{e x p}$ is the Monte Carlo expectation for energy bin $i$, which includes both the signal and background components. $N_{i}^{o b s}$ is the observed number of data events in energy bin $i$. The second sum adds penalty terms for systematic uncertainties associated with the $m$ sources of uncertainty. In that sum, the expectation is that the default values, $\alpha_{j}$ of the parameters $j$ are known, and any variation from those values $\epsilon_{j}$ should be zero. The variation in these parameters is constrained by the uncertainty in the parameters.

The number of expected events, $N_{i}^{e x p}$ in the Near Detector for a given energy bin $i$ is

$$
N_{i}^{e x p}=N_{i}^{0} \times\left(1+\sum_{j}^{m} f_{j}^{i} \epsilon_{j}\right)
$$

where $N_{i}^{0}$ is the expected number of events without changing any of the default values for the systematic parameters. This last factor adjusts the number of expected events in energy bin $i$ for each systematic parameter $j$. The $f_{i}^{j}$ is the fractional change in the number of expected Monte Carlo events in energy bin $i$ due to a change $\epsilon_{j}$ in the value of the parameter $j$. The simplest example of this would be the overall normalization. A change in the overall normalization of bin $i$ of $5 \%$ will cause a $5 \%$ increase in the number of events seen in energy bin $i$. This means that the fractional change due to overall normalization is 1 .

The Marquardt fitting method depends on knowing the values of $f_{j}^{i}$ for all systematic parameters in all energy bins. The main advantage of the Marquardt method is its speed. By knowing the relationship between changes in a parameter and the corresponding change in bin content, one does not have to individually 
change the weight given to every Monte Carlo event in their sample. One only needs to change the values of individual bin contents.

To determine $f_{j}^{i}$ we change the value of $\alpha_{j}$ by $n \sigma$, where $n=-3,-2, \ldots, 2,3$ and we find the ratio $r_{j}^{i}=N_{j}^{\text {adjust }, i} / N_{j}^{0, i}$ for each change. $N_{j}^{\text {adjust }, i}$ is the number of events predicted in the Monte Carlo after changing $\alpha_{j}$ by the specified amount. We then plot these ratios as a function of $\alpha_{j}+n \sigma_{j} / \alpha_{j}$. A line is fit to the points and the slope of the line is $f_{j}^{i}$. The expectation is that a change in the value of one of the systematic parameters will cause a linear change in $N_{i}^{0}$. The linearity of the parameters chosen is discussed in the next section.

It takes approximately a night of processing to produce a file with all the fractional changes, $f_{j}^{i}$, needed to perform the fit. After this is complete, fitting for multiple systematic parameters in the Near detector is extremely fast. In the next section we briefly present the systematic parameters used to fit the Near detector data.

\subsection{Systematic Parameters used in the NDFit}

The basic categories of systematic parameters in this analysis are beam, crosssection, and reconstruction systematics. In the next section a comprehensive study is performed on the effects of five different groups of systematic uncertainties. A certain set of systematic parameters must be chosen in which to fit for in the Near detector. Since an infinitely long list of systematics cannot be used we choose a list of parameters which have large impacts on the energy spectra. The next four subsections detail what parameters have been chosen in each category and why they are used. 


\subsubsection{Beam Systematics}

Since there was a dedicated study [101] to correct for any uncertainties associated with our neutrino flux model, as described in Chapter 5, the Near detector data is not fit using beam related parameters. Systematic studies are later performed where these corrections are shifted by their $\pm 1 \sigma$ errors to see the effect on the oscillation parameters. Figure 8.1 shows the effect on the NC-like and CC-like energy spectra of changing the neutrino flux corrections by their $\pm 1,2,3 \sigma$ errors. Since this is an applied correction, fitting for it in the Near detector would be a redundancy, and it is unlikely that fitting for neutrino flux correction parameters will yield results that are more rigorous than the actual study.
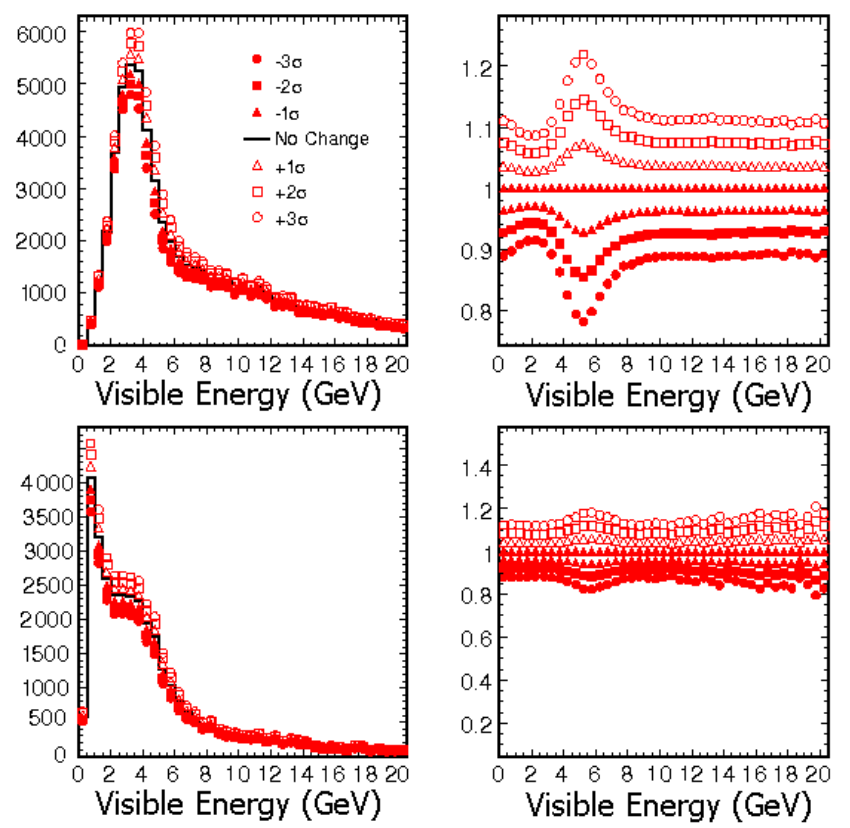

Figure 8.1: The effect of changing the neutrino flux corrections by their $\pm 1,2,3 \sigma$ errors. The top left plot is the charged current and the bottom left is the neutral current energy spectra. The two plots on the right are the ratio of the nominal spectrum divided by the spectra with the $\pm 1,2,3 \sigma$ changes. 


\subsubsection{Cross-section Systematics}

For cross-section systematics, we look at different sets of parameters available from the NEUGEN3 neutrino event generator that was described briefly in Chapter 4. Work has been done by individuals directly involved in NEUGEN3 development [108] in order to identify which of the numerous simulation parameters are useful to allow in our Near detector fit.

Sources of uncertainty which either have been demonstrated [108] to have little effect on MINOS measurements or which do not contribute directly to the CC-like and NC-like energy distributions are not considered. For a statistics limited analysis, as our data will be in the Far detector, the most important NEUGEN3 parameters and their estimated uncertainties are:

1. Quasi-elastic $m_{A}$ : The quasi-elastic axial vector mass with a value of 0.99 . This is essentially the only free parameter in the quasi-elastic cross section from free nucleons and a $15 \%$ uncertainty has been taken.

2. Resonance $m_{A}$ : The resonance axial vector mass with a value of 1.12 . This is the main free parameter in the Rein-Seghal [94] model and governs the overall normalization and $\mathrm{q}^{2}$ distribution shape in resonance production reactions. In NEUGEN3, equality with the quasi-elastic form factor is assumed and a $15 \%$ uncertainty is used.

3. $\mathbf{k n o}_{i j k}$ Factors: One of the principle challenges in building a comprehensive cross section model over this energy range is bridging the gap between perturbative and non-perturbative models of neutrino interactions, specifically in deep inelastic scattering (DIS). In NEUGEN3 this difficulty is addressed by a data-driven approach in which 16 free parameters $\left(\mathrm{kno}_{i j k}\right)$ are used to fit the exclusive channel data as well as the total cross section. The approach assumes that $\sigma_{\text {total }}=\sigma_{\text {quasi-elastic }}+\sum_{k}\left(\sigma_{\text {resonance }}^{k}+\mathrm{kno}_{i j k} \times \sigma_{D I S}^{k}\right)$. The index 


\begin{tabular}{|c|c|c|}
\hline kno Parameter & Nominal Value & $1 \sigma$ Error \\
\hline \hline kno $_{12}$ & 0.1 & 0.1 \\
\hline kno $_{122}$ & 0.3 & 0.1 \\
\hline kno $_{13}$ & 1.0 & 0.2 \\
\hline kno $_{123}$ & 1.0 & 0.2 \\
\hline kno $_{212}$ & 0.1 & 0.1 \\
\hline kno $_{222}$ & 0.3 & 0.1 \\
\hline kno $_{213}$ & 1.0 & 0.2 \\
\hline kno $_{23}$ & 1.0 & 0.2 \\
\hline
\end{tabular}

Table 8.1: The nominal values and $1 \sigma$ errors on the eight kno parameters considered in this analysis.

$k$ refers to the multiplicity of the hadronic system. In NEUGEN3 only parameters corresponding to the $k=2$ and $k=3$ states are adjusted. The $k=1$ state is elastic scattering and does not contribute here to our DIS model. Values of $k>3$ have very little contribution to our Monte Carlo and can be ignored. The index $i$ is equal to 1 for charged current and 2 for neutral current interactions. The index $j$ refers to the four combinations of neutrino/anti-neutrino interacting with either a proton or neutron. We consider only neutrino on proton/neutron because it is more accurate error estimate to scale the overall anti-neutrino components of the energy spectra by a factor rather than change any of the kno parameters [108]. This leaves 8 different kno parameters for us to use. The values of these parameters affect the NEUGEN3 prediction and the cross section for exclusive channels, the total cross section, and the shape of kinematic distributions. The values for these kno parameters are given in Table 8.1

How the simulation parameters are used must be discussed. Since the two axial mass parameters should affect only a small number of bins in reconstructed energy and $\mathrm{y}_{b j}$, defined as the fraction of the neutrino energy deposited by hadronic contributions, the error model can be considered an absolute cross section error 
on just these interactions, and not an estimate of the fit to the shape of the cross section in either energy or or $\mathrm{y}_{b j}$, nor any other kinematic variable. Due to this, we combine the $m_{q e l, A}$ and $m_{r e s, A}$ into one parameter called "CCMA". Since these two parameters have the same $1 \sigma$ error of $15 \%$, they will be changed in unison.

Of the 16 different $\mathrm{kno}_{i j k}$ parameters, only neutrino on nucleon and not antineutrino on nucleon are used because it was determined that fixing an absolute error on the anti-neutrino component of the energy spectra was a more accurate [108] measure of the anti-neutrino error. This leaves $8 \mathrm{kno}_{i j k}$ parameters which are combined for the same reasons as $m_{q e l, A}$ and $m_{r e s, A}$ into groups of two which are changed in unison. Each kno parameter should change simultaneously with the corresponding $\mathrm{kno}_{i j k}$ with the same interaction type and particle multiplicity, that is the $i$ and $k$ index. The four combinations will be referred to as $\mathrm{kno}_{112122}, \mathrm{kno}_{113123}, \mathrm{kno}_{212222}$, and $\mathrm{kno}_{213223}$. The effect on the NC-like and CC-like energy spectra of changing these five parameters by $\pm 1,2,3 \sigma$ is documented in Figures B.1 through B.5.

\subsubsection{Reconstruction Systematics}

For reconstruction errors, one takes into account systematics that can be caused by the reconstruction software and calibration on reconstructed quantities and selection of events. Since the energy spectra are defined by the energy contained within reconstructed showers and tracks, we chose these two as important systematics that can vary [109, 110]. Our reconstruction cannot necessarily account for all errors in counting protons on target and errors in the steel masses of the detectors, so the overall normalization is also fit [112]. Last, if there exists some bias in our reconstruction and hence selection of events, then the respective backgrounds in the CC-like and NC-like energy spectra will be biased. Included in our fit is a scaling on the corresponding background to the CC-like [111] and NC-like [113] energy spectra. The five reconstruction parameters are listed in Table 8.2. 


\begin{tabular}{|c|c|c|}
\hline Reconstruction Systematic & Nominal Value & $1 \sigma$ Error \\
\hline \hline Shower Energy & 1.0 & 0.11 \\
\hline Track Energy & 1.0 & 0.02 \\
\hline Normalization & 1.0 & 0.03 \\
\hline NC Background & 1.0 & 0.5 \\
\hline CC Background & 1.0 & 0.05 \\
\hline
\end{tabular}

Table 8.2: The nominal values and $1 \sigma$ errors on the five reconstruction systematic parameters fit for in the Near detector.

The effect on the NC-like and CC-like energy spectra of changing these five parameters by $\pm 1,2,3 \sigma$ is documented in Figures B.6 through B.10.

\subsubsection{Parameters used in Near detector Fit}

It is crucial that the $f_{j}^{i}$ relationship is approximately linear for the fitted systematic parameters or else it will skew the results of the Near Detector fit. It is also important to use parameters that have an noticeable effect on the CC-like and NC-like energy spectra. Using this criteria the chosen parameters used in the Near Detector are given in Table 8.3 and discussed in the previous sections. The first column indicates whether the parameter is from the NEUGEN3 simulation or the reconstruction, the second gives the name, the third is the default value, the fourth is the $1 \sigma$ uncertainty and the fifth indicates how we will be fitting for the parameter. The fifth column needs more explaining. If it says "Marquardt" this means that particular parameter will be fit using the Marquardt prescription, if it says "Loop" this means that a loop over an acceptable range of values for the parameter will be performed to find the best fit. Notice that only the shower energy and track energy will be fit using a loop, because their fractional changes, $f_{j}^{i}$ are not linear and hence would bias the fit.

It has been commented that in order to fit for certain systematic parameters in the Near detector using the Marquardt fitting, there must exist a linear relation- 


\begin{tabular}{|c|c|c|c|c|}
\hline Parameter Type & Parameter Name & Default Value & $1 \sigma$ error & Fit Type \\
\hline \hline Simulation & CCMA & 1.0 & 0.15 & Marquardt \\
\hline Simulation & kno $_{112122}{ }^{*}$ & 1.0 & $1.0,0.33$ & Marquardt \\
\hline Simulation & kno $_{113123}{ }^{*}$ & 1.0 & $1.0,0.33$ & Marquardt \\
\hline Simulation & kno $_{21222}$ & 1.0 & 0.2 & Marquardt \\
\hline Simulation & kno $_{21322}$ & 1.0 & 0.2 & Marquardt \\
\hline Reconstruction & Shower Energy & 1.0 & 0.11 & Loop \\
\hline Reconstruction & Track Energy & 1.0 & 0.02 & Loop \\
\hline Reconstruction & Normalization & 1.0 & 0.03 & Marquardt \\
\hline Reconstruction & CC Background & 1.0 & 0.05 & Marquardt \\
\hline Reconstruction & NC Background & 1.0 & 0.50 & Marquardt \\
\hline
\end{tabular}

Table 8.3: Systematic Parameters to be fit in the Near Detector. ${ }^{*}=$ knor112122 and knor113123 consist of two parameters with two different errors. knor112 and knor113 have an error of 100\%, knor122 and knor123 have an error of $33 \%$.

ship between the percentage change in the systematic parameter and the percentage change in the contents of each energy bin. We have documented the linearity of this relationship [114]. Two examples showing this linear relationship are in Figures 8.2 and 8.3 which plot the percentage change in the parameter versus percentage change in bin content from $0-5 \mathrm{GeV}$ in bins of width $0.5 \mathrm{GeV}$ for charged current events for CCMA and kno112122 and the normalization parameter for neutral current events in Figure 8.4. As noted before, we do not fit the shower and track systematic parameters with the Marquardt method because they do not exhibit a linear relationship between parameter changes and bin content changes, this is illustrated in Figure 8.5. For the shower and track systematics, a loop is performed over an acceptable range of values, and the changes are applied separately from the Marquardt method. These plots are fit with a straight line to point out their acceptable linearity. The slope of the line is equal to the $f_{i}^{j}$ value for that energy bin. 

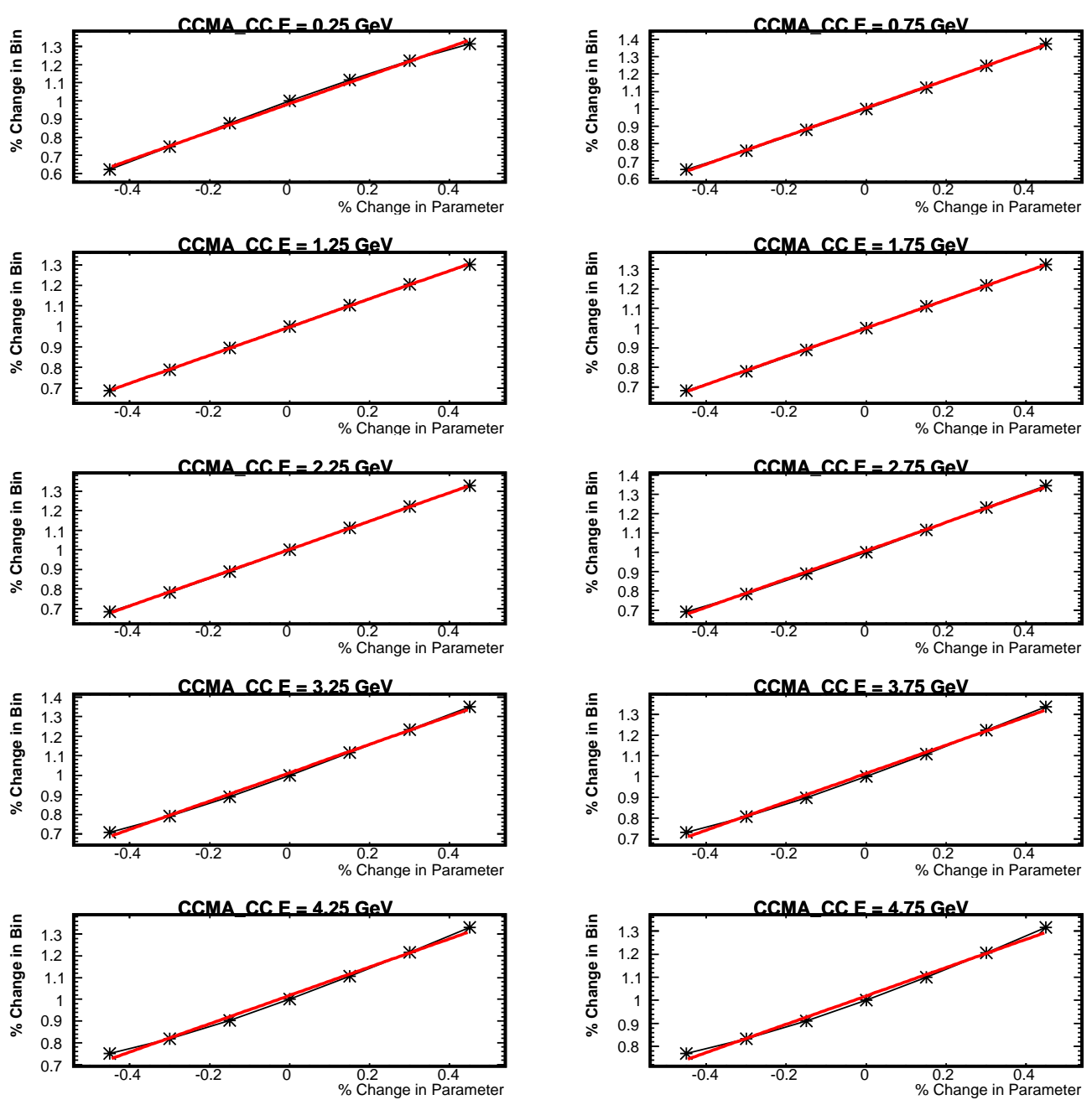

Figure 8.2: Fractional Change in the Neugen Parameter CCMA versus Fractional Bin Content Change for CC-like events from 0-5 GeV Energy Bins 

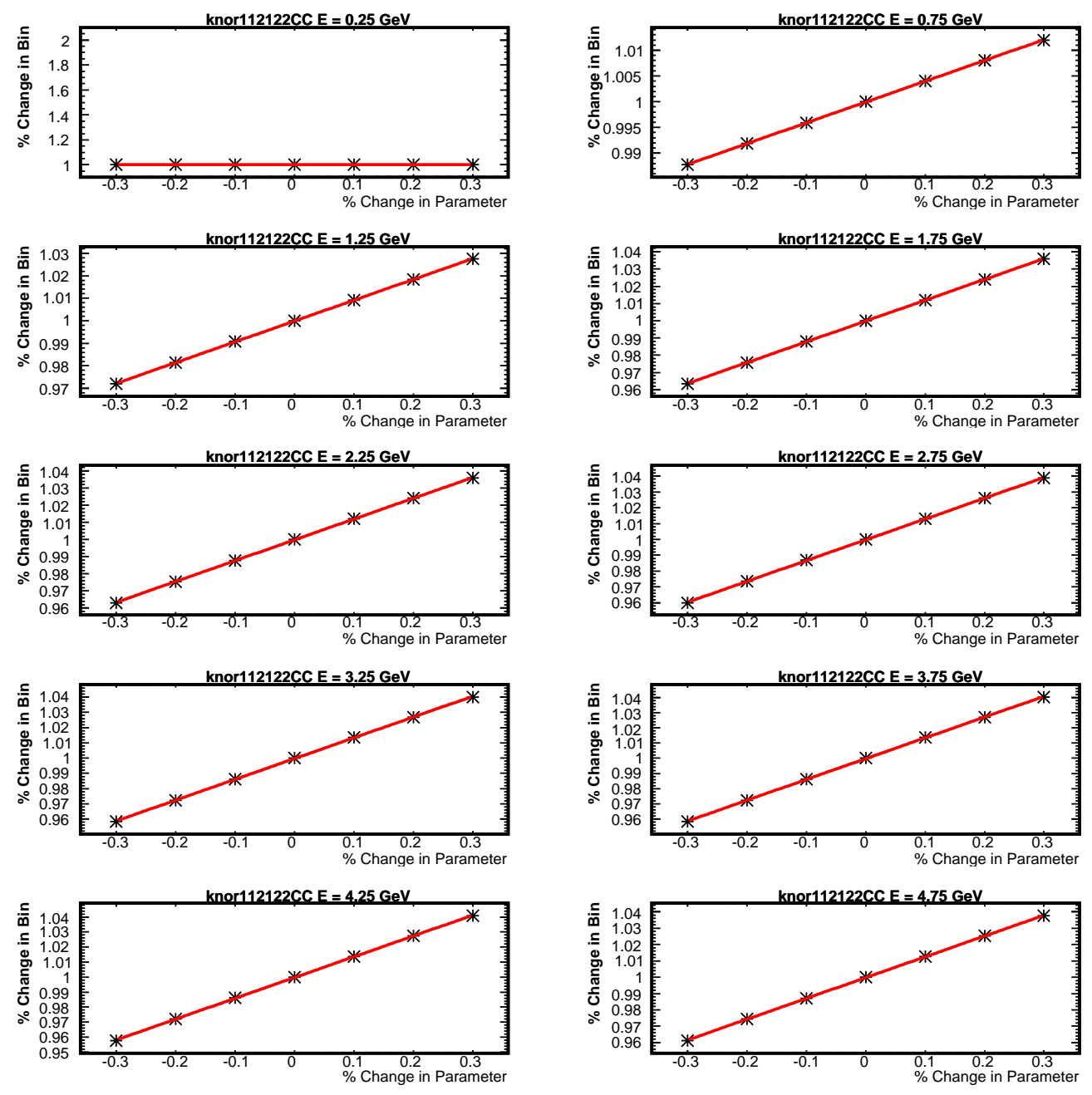

Figure 8.3: Fractional Change in the Neugen Parameter knor112122 versus Fractional Bin Content Change for CC-like events from 0-5 GeV Energy Bins 

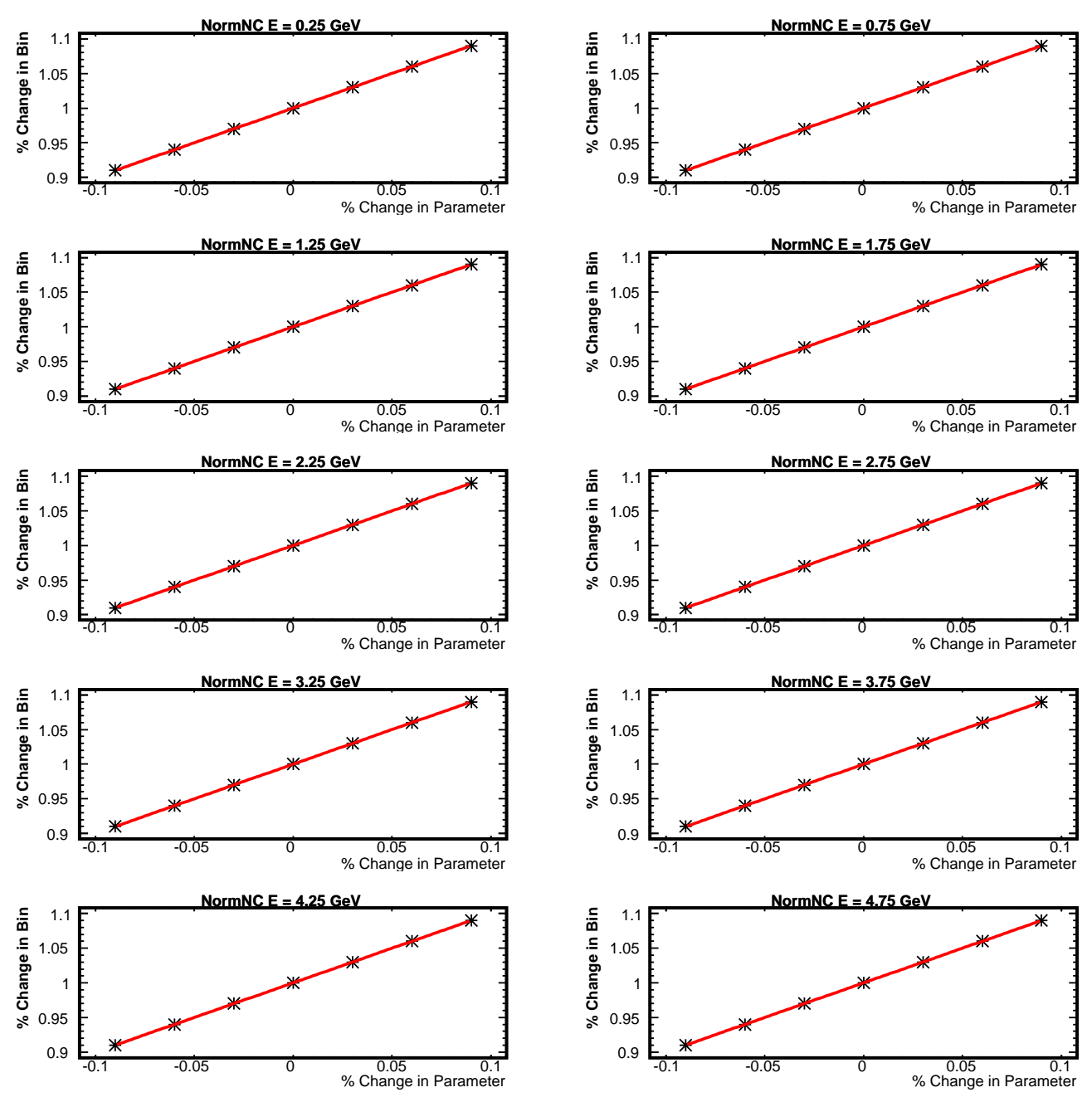

Figure 8.4: Fractional Change in Normalization versus Fractional Bin Content Change for NC-like events from 0-5 GeV Energy Bins 

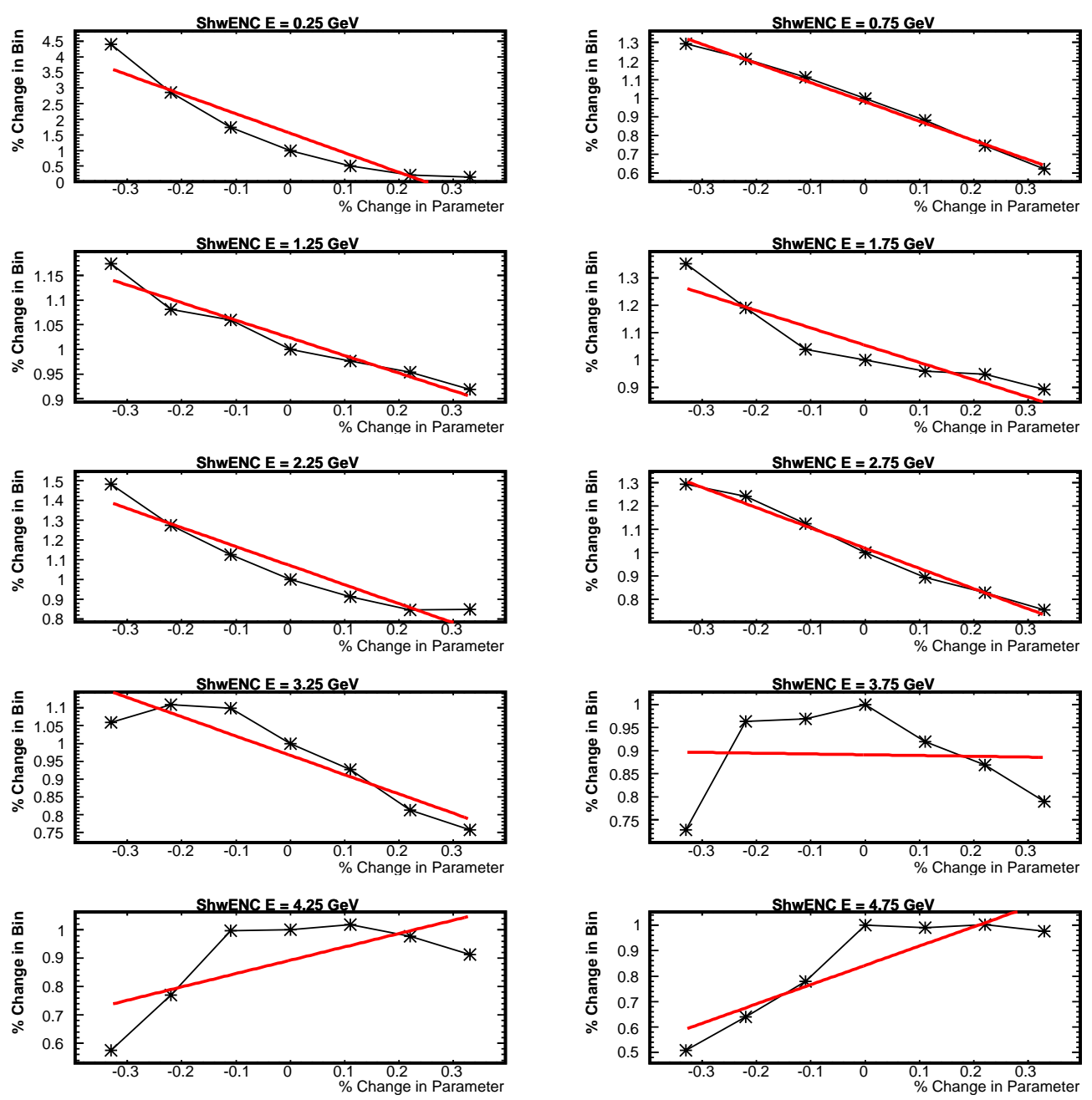

Figure 8.5: Fractional Change in Shower Energy versus Fractional Bin Content Change for NC-like events from 0-5 GeV Energy Bins 


\subsection{Systematic Studies}

In order to show that the Marquardt style fitting is robust, various systematic studies must be performed to show that the results of the oscillation fitting routine are not biased. Simulated data is generated from Monte Carlo and the spectra are oscillated for parameters equal to $\Delta m^{2}=0.0027 \mathrm{eV}^{2} / \mathrm{c}^{4}, \sin ^{2}(2 \theta)=1.0$, and $f_{\text {sterile }}=0.1$. We select the total exposure to be $2.5 \times 10^{20}$ protons on target since that is approximately the data exposure will be used in this analysis.

First, it must be shown that the fitting routine can extract these oscillation parameters in the absence of systematic shifts to cross-section, beam, and reconstruction parameters. Figure 8.6 shows the Near detector energy spectra with our simulated data in black, nominal Monte Carlo in blue and the result of the Near detector fit in red. The red completely overlaps the blue Monte Carlo spectra because there exist no systematic shifts in this plot, hence the fit does nothing. The bottom two plots show the ratio of the data to nominal Monte Carlo in blue and the ratio of the data to the fit Monte Carlo in red. One sees there is perfect agreement in the absence of systematic parameter shifts, as expected. Figure 8.7 shows the Far detector oscillated data in black, the Monte Carlo prediction in blue, the result of the oscillation fit in red, and in magenta the tau appearance component of the spectrum. One can see from the bottom two ratios that the fit of oscillated data to fit Monte Carlo in red is one. The results in parameter space for this fit are given in Figure 8.8. The top plot is the two dimensional contour for $\Delta m^{2}$ versus $f_{\text {sterile }}$. The true input value for the oscillation parameters is given by the clear star and the best fit point is the black star. Notice that they lie on top of each other. The corresponding black, red, and blue contours correspond to the $69 \%, 90 \%$, and $99 \%$ confidence levels around the best fit point. We see that this simple test correctly measures the oscillation parameters. 

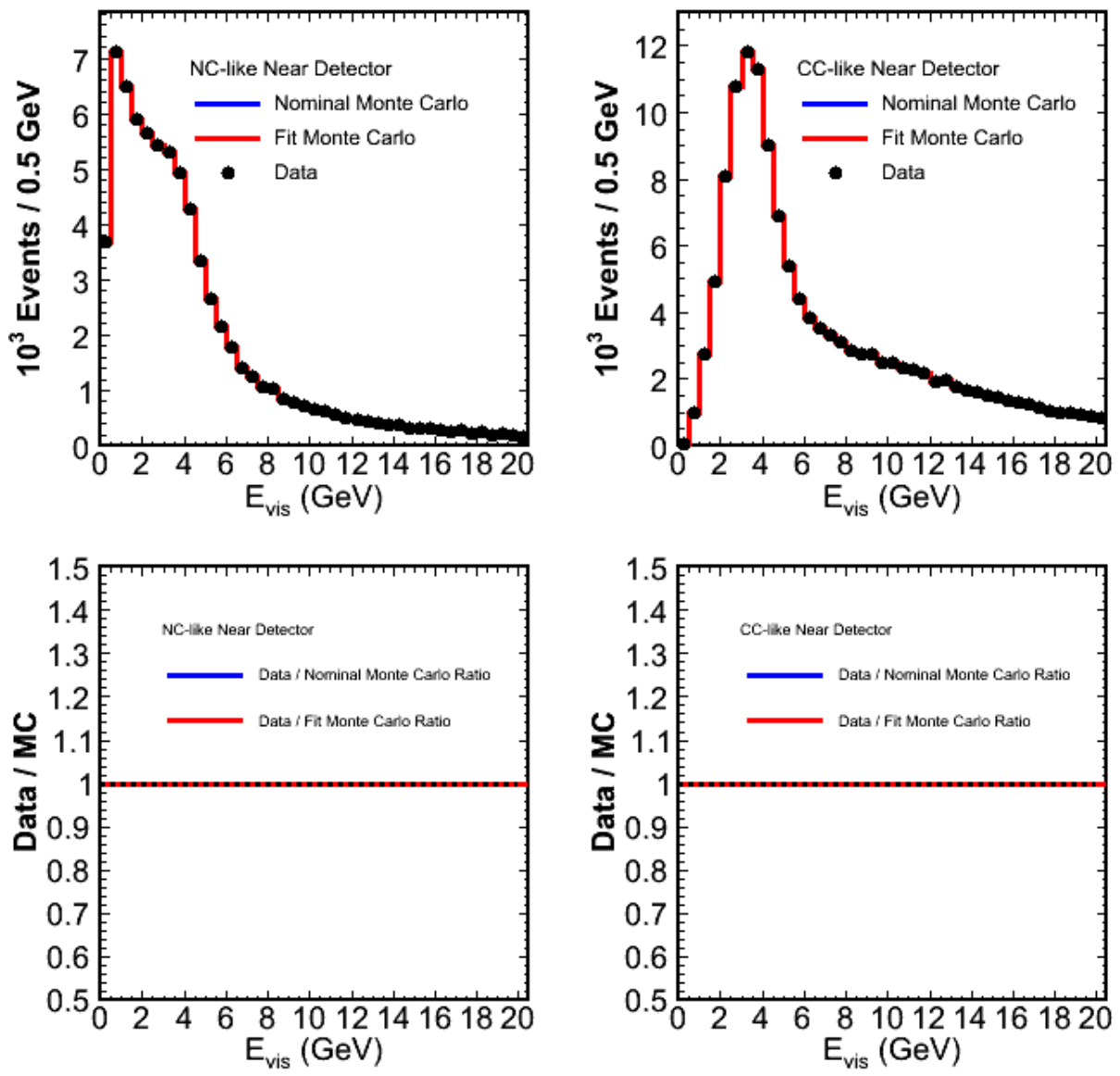

Figure 8.6: Near Detector NC-like and CC-like energy spectra in the top left and top right plots respectively. The bottom left and right plots are the ratios of simulated data over nominal and fit Monte Carlo. There are no systematic shifts in these spectra 

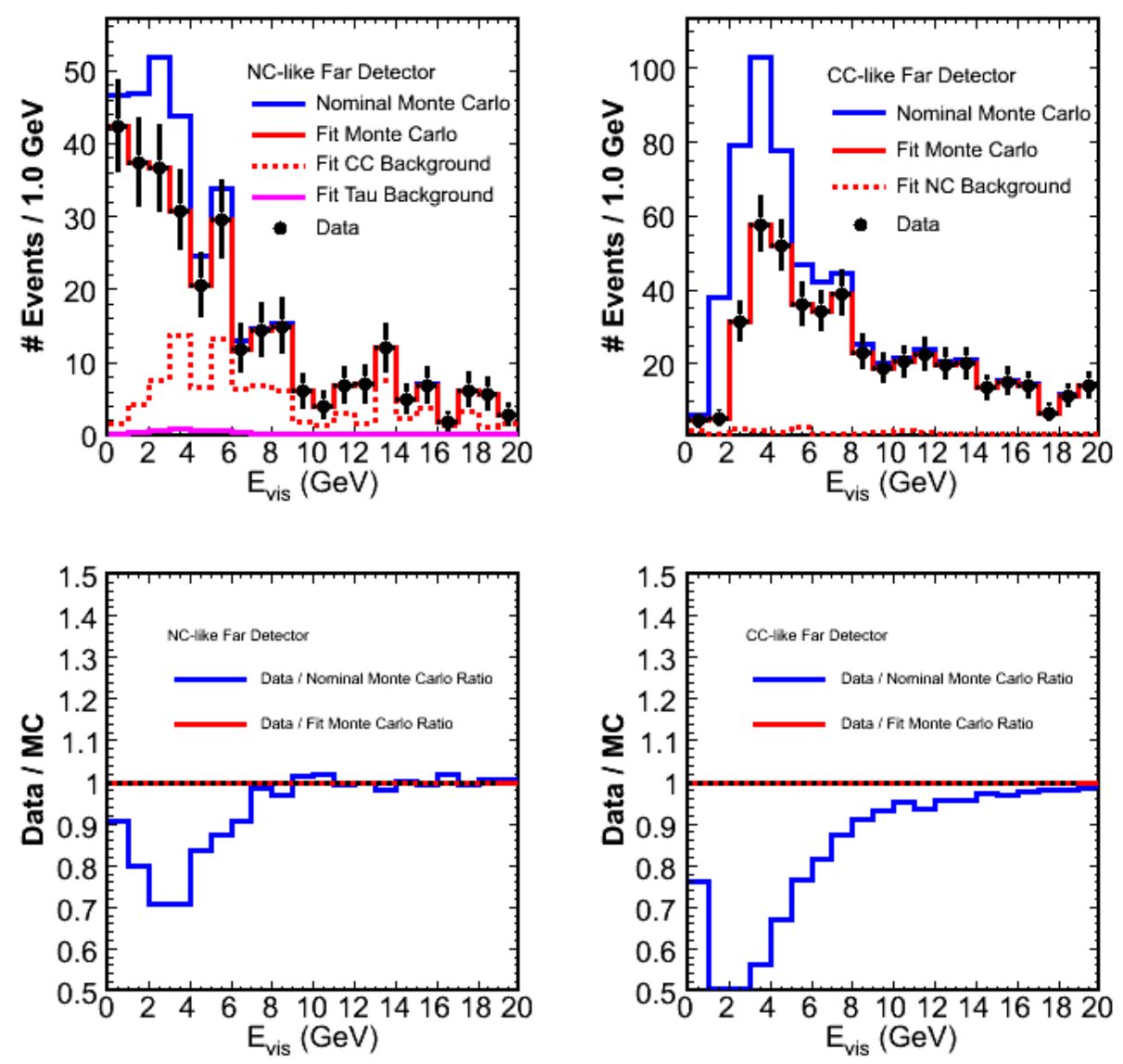

Figure 8.7: Far Detector NC-like and CC-like energy spectra in the top left and top right plots respectively. The bottom left and right plots are the ratios of simulated data over nominal and fit Monte Carlo. There are no systematic shifts in these spectra, only effects from oscillations. 

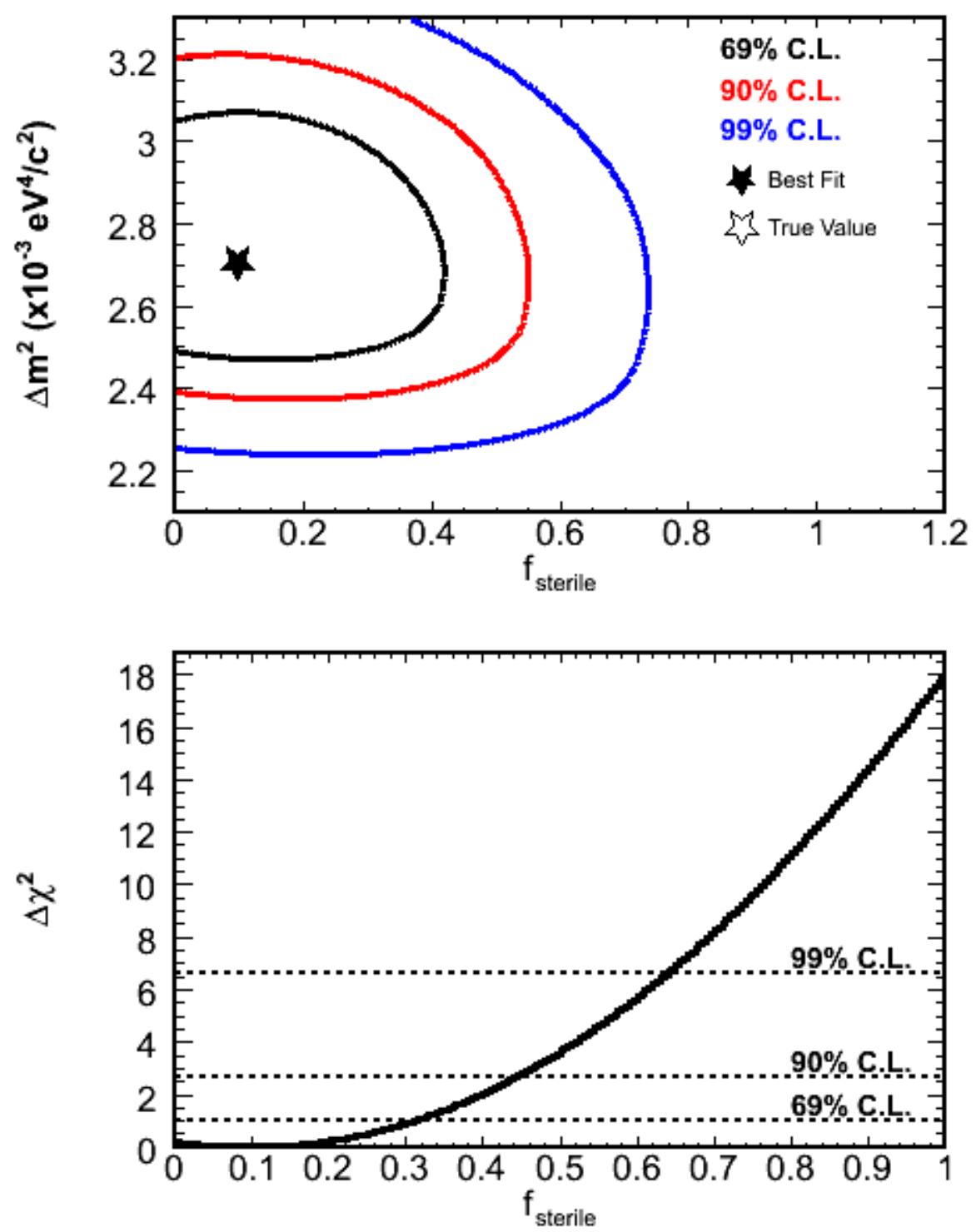

Figure 8.8: The top plot is the $69 \%, 90 \%, 99 \%$ 2-D confidence level contours for $f_{\text {sterile }}$ vs $\Delta m^{2}$ while the bottom plot is the 1-D confidence level contours for $f_{\text {sterile }}$ for no systematic shifts. 
Five sets of systematic studies are performed. Each category of systematic studies is suppose to test the robustness of our fitting method. We categorize them as follows:

1. Systematic Study I The 10 parameters that will be fit for in the Near detector, listed in Table 8.3, are individually changed by $\pm 1 \sigma$ for both the Near and Far detector simulated data before proceeding with the oscillation analysis.

2. Systematic Study II The 10 parameters that we will be fitting for in the Near detector are individually changed by $\pm 1 \sigma$ for both the Near and Far detector simulated data, but we choose not to fit for the changed parameter in the Near detector. This reduces the number of parameters we fit for in the Near detector to 9 .

3. Systematic Study III An independent list of systematic changes is defined and applied to both the Near and Far detector simulated data but when we perform the oscillation analysis only the parameters listed in Table 8.3 are fit for in the Near detector.

4. Systematic Study IV Four different combinations in which multiple systematic parameters are changed are defined to evaluate the effect of multiple systematic errors on the analysis.

5. Systematic Study V Last, the original 10 parameter are changed by $\pm 1 \sigma$ only in the Far detector, and not in the Near, and then we proceed with the analysis. Since this particular study has no information from the Near detector, one expects this to be the worst case scenario. 


\subsubsection{Systematic Study I}

The first set of systematic studies individually changes the parameters that will be fit in the Near Detector by $\pm 1 \sigma$ in both the Near and Far detectors. These parameters are defined in Table 8.3. Since we are fitting for these parameters in the Near detector, very small changes to the best fit oscillation parameters are expected. This test is mainly to see if the fitting routine is working properly. The results of the oscillation fits are shown for $f_{\text {sterile }}$ in Figure 8.10 and $\Delta m^{2}$ in Figure 8.11. The systematic parameter changed is listed to the left of each plot and the points to the right show the best fit with a black dot and the error bars are the $1 \sigma$ errors on the measurement. The superimposed dashed red line is the true input value for the two oscillation parameters. Notice that the true oscillation parameters are always measured correctly to within $69 \%$ confidence level for all the systematic shifts. These plots will have a similar format for all five systematic studies. As an example the Near detector fit with CCMA changed by $-1 \sigma$ is shown in Figure 8.9. Notice in the bottom two plots the ratio of the simulated data to the fit Monte Carlo in red is flat at one, meaning the fit was able to resolve the differences between simulated data and Monte Carlo. 

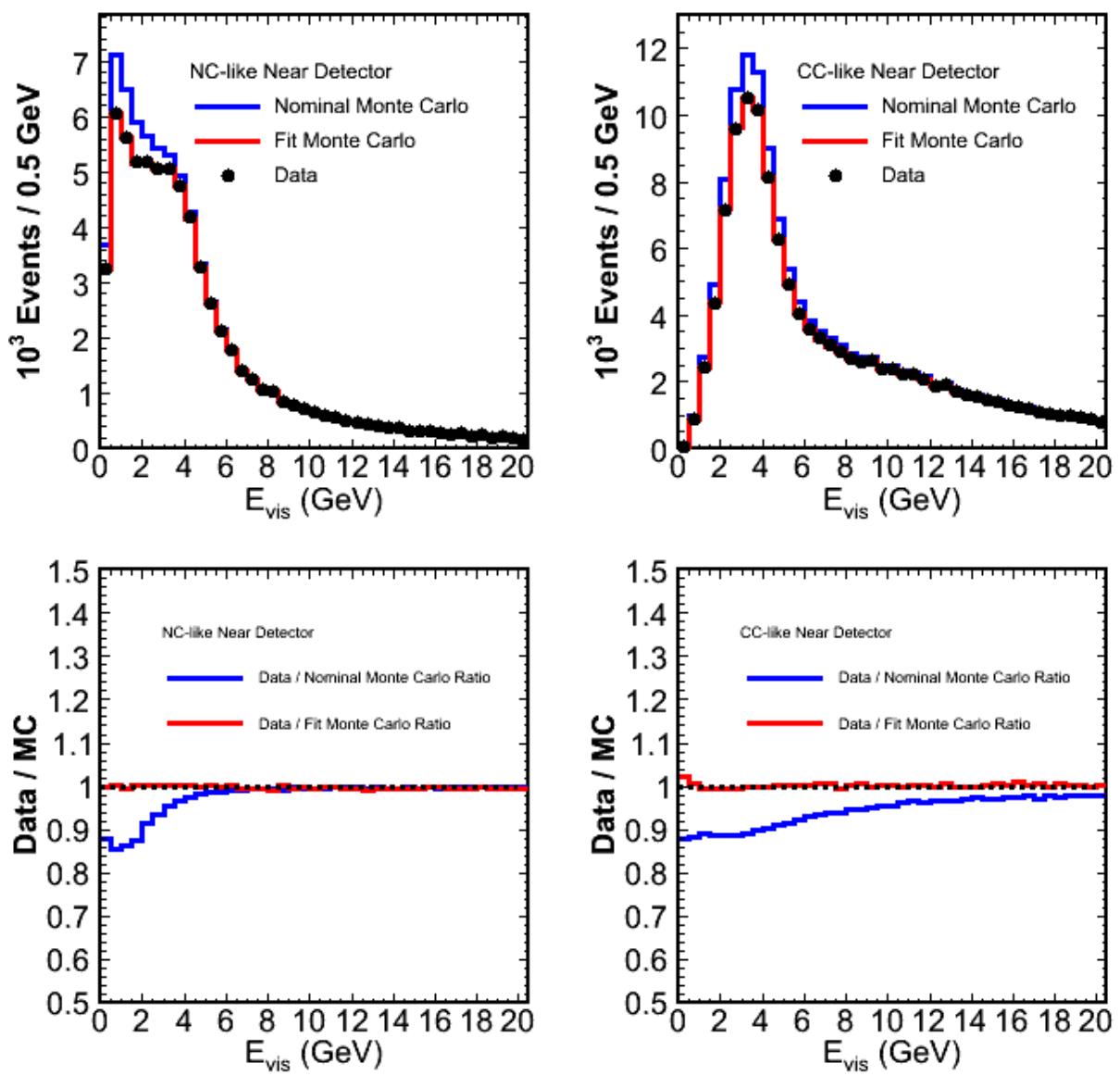

Figure 8.9: The top left is the neutral current energy spectrum and the top right is the charged current energy spectrum. The black dots are simulated data with a $-1 \sigma$ change in CCMA applied. The blue is the nominal Monte Carlo and the red is the result of the best fit. The bottom two plots show the ratio of simulated data to nominal Monte Carlo in blue and simulated data to best fit Monte Carlo in red. 


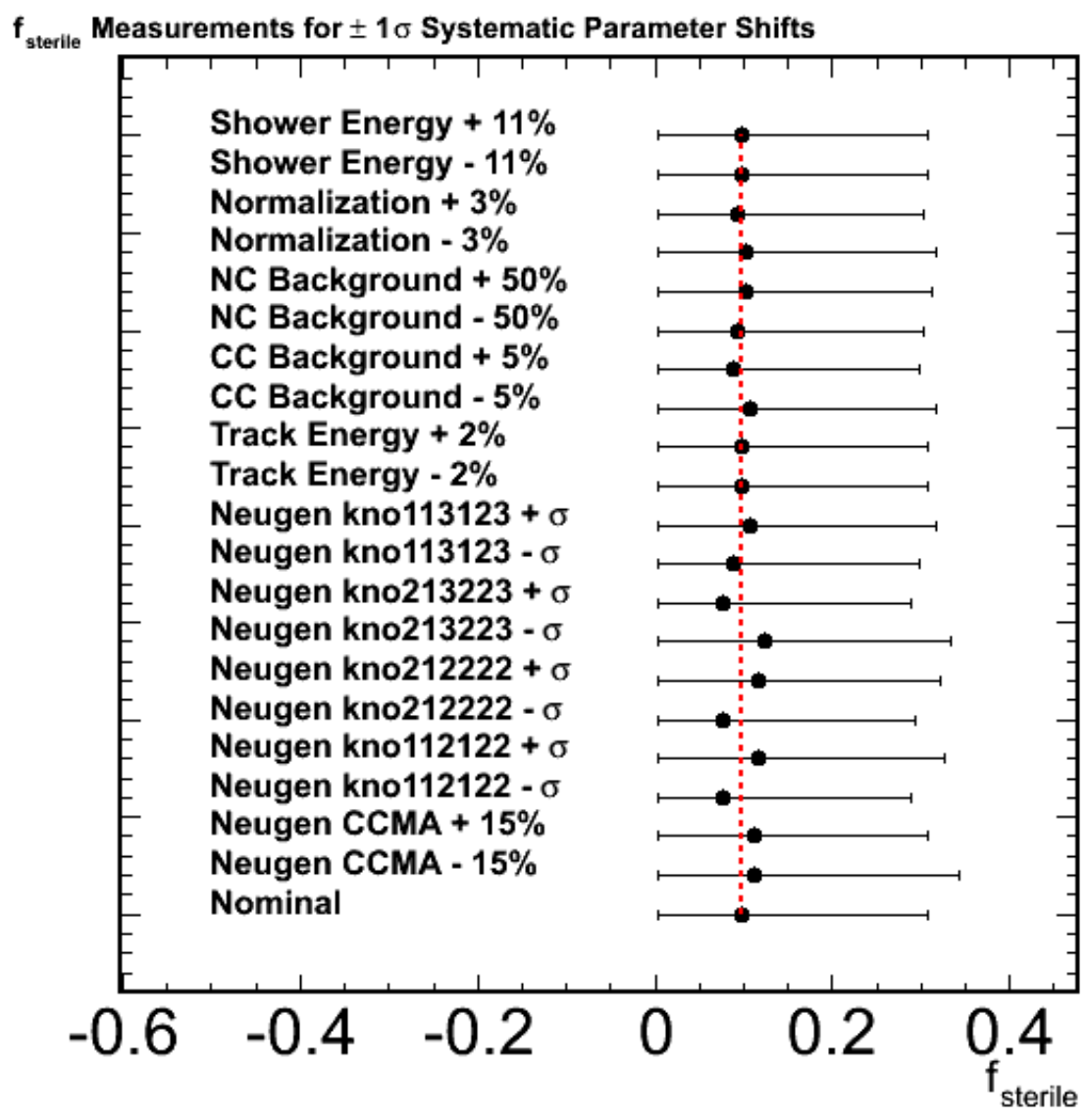

Figure 8.10: Summary of best fit values and $\pm 1 \sigma$ error on $f_{\text {sterile }}$ for systematic study I. 


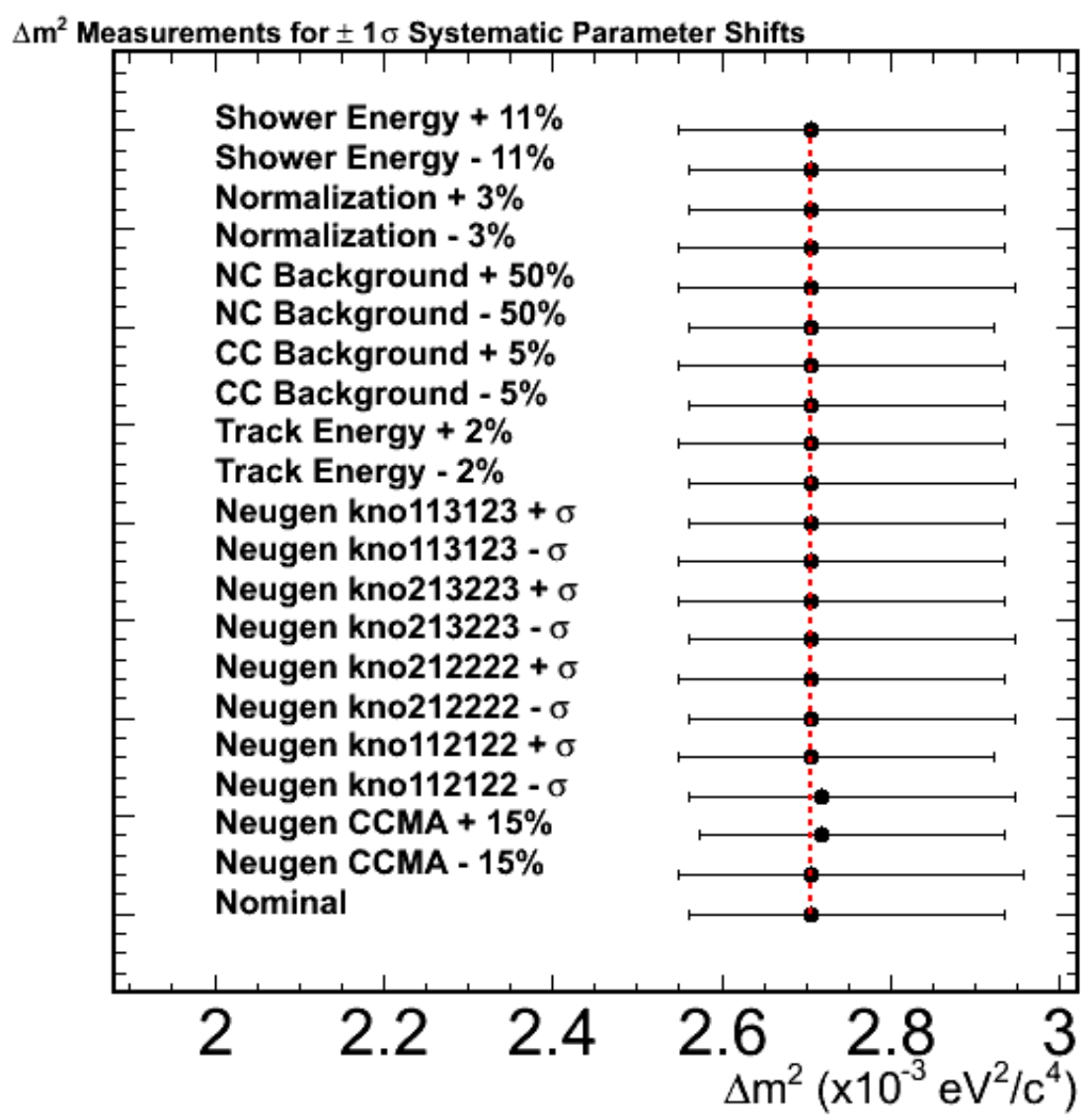

Figure 8.11: Summary of best fit values and $\pm 1 \sigma$ error on $\Delta m^{2}$ for systematic study I. 


\subsubsection{Systematic Study II}

The second set of systematic studies consists of individually changing the parameters fit in the Near detector by $\pm 1 \sigma$ in both the Near and Far detectors, and then a fit would be performed only for the other nine parameters in the Near detector. For instance, if one takes the first parameter in Table 8.3, which is CCMA, it would be changed by $\pm 1 \sigma$ in both the Near and Far detector simulated data and the fit uses only the other nine parameters in the Near detector. This is done for all 10 parameters in the table. This tests the ability of the fitting method to resolve differences between data and Monte Carlo for parameters that we will not be fitting for.

The results of the oscillation fits are shown for $f_{\text {sterile }}$ in Figure 8.12 and $\Delta m^{2}$ in Figure 8.13. The systematic parameter changed is listed to the left of each plot and the points to the right show the best fit with a black dot and the error bars are the $1 \sigma$ errors on the measurement. All fits recover the correct oscillation parameters, although the shift in CCMA is larger than all the others. This is because CCMA has a large impact on the energy spectra as shown in Figrure B.1 and it is difficult to reproduce its effects with the other nine parameters. 


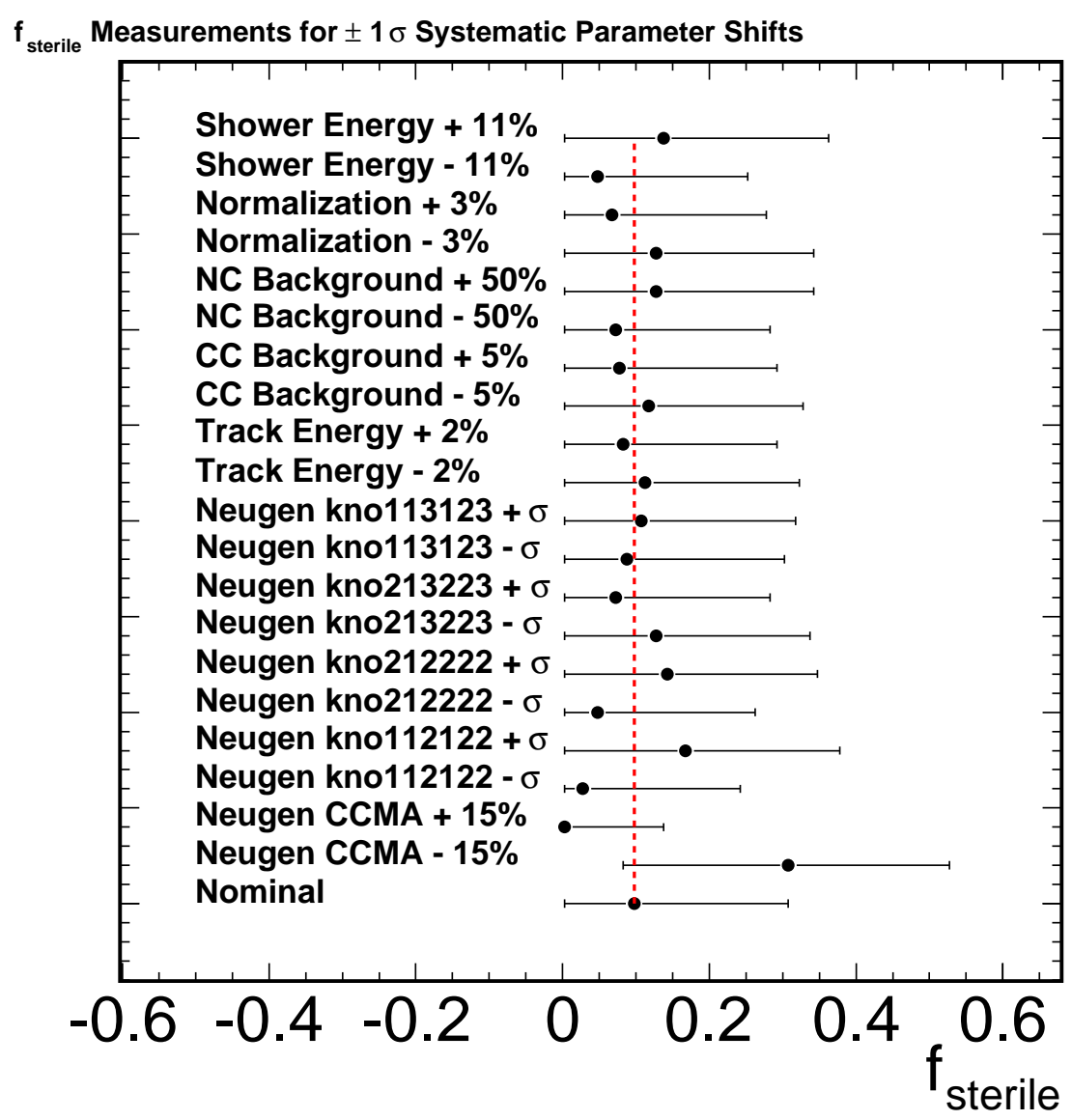

Figure 8.12: Summary of best fit values and $\pm 1 \sigma$ error on $f_{\text {sterile }}$ for systematic study II. 


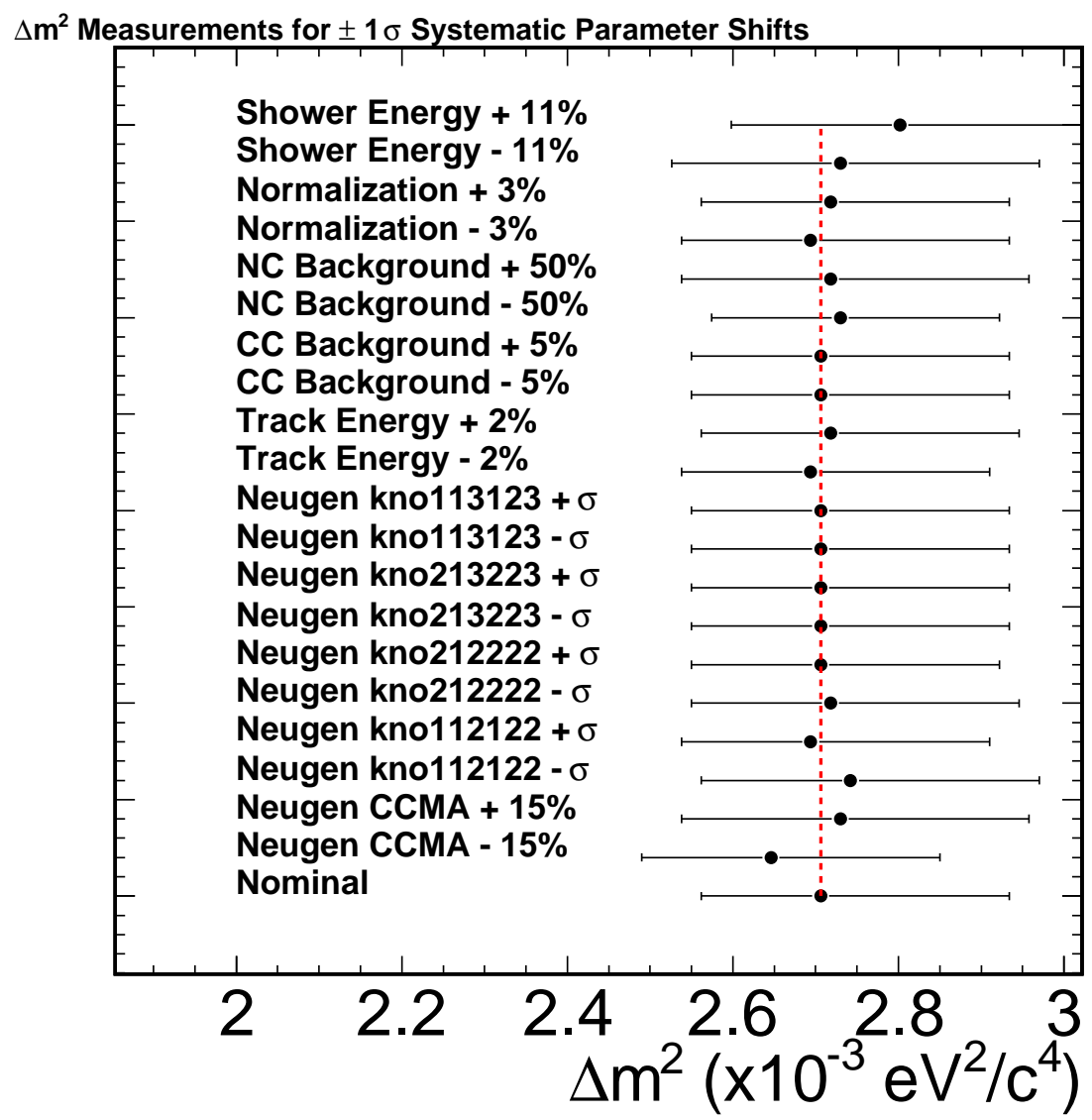

Figure 8.13: Summary of best fit values and $\pm 1 \sigma$ error on $\Delta m^{2}$ for systematic study II. 


\subsubsection{Systematic Study III}

The third set of systematic studies addresses systematic parameters that will not be fit for in the Near detector, but could potentially bias the oscillation fit. The parameters listed in Table 8.4 were changed by $\pm 1 \sigma$ for both the Near and Far detector simulated data, but the fit only uses the parameters listed in Table 8.3.

The first parameter deals with incomplete modeling of the anti-neutrino cross-section, and so a scaling of $\pm 20 \%$ is introduced to the anti-neutrino component of the spectra. The Low Completeness Fraction parameter is a scaling on events that are classified as low completeness. This could be a potential bias because the lowest energy bins in the neutral current energy spectrum in the Near detector contain these types of events, but the Far detector does not.

\begin{tabular}{|c|c|c|c|c|}
\hline Parameter Type & Parameter Name & Default Value & $1 \sigma$ Error & Fit Type \\
\hline \hline Cross-section & Anti-Neutrino Scaling & 1.0 & 0.2 & Marquardt \\
\hline Reconstruction & Low Completeness Fraction & 1.0 & 0.219 & Marquardt \\
\hline Cross-section & NC Scale & 1.0 & 20 & Marquardt \\
\hline Cross-section & NC Scale(E) & 1.0 & $*$ & Marquardt \\
\hline Simulation & Not including Taus & 1.0 & N/A & Marquardt \\
\hline Calibration & Relative Cal Err & 1.0 & 0.02 & Loop \\
\hline Simulation & Shower Offset 1 & 0.0 & $150 \mathrm{MeV}$ & Loop \\
\hline Simulation & Shower Offset 2 & 0.0 & $100 \mathrm{MeV}$ & Marquardt \\
\hline Simulation & Shower Offset 3 & 0.0 & $50 \mathrm{MeV}$ & Marquardt \\
\hline Beam & SKZP & 0.0 & 1.0 & Marquardt \\
\hline
\end{tabular}

Table 8.4: Systematic Parameters to be fit in the Near Detector. ${ }^{*}=20 \%$ for $\mathrm{E}<1.5$ $\mathrm{GeV}, 10 \%$ for $1.5<\mathrm{E}<3.0 \mathrm{GeV}$, and $5 \%$ for $\mathrm{E}>3.0 \mathrm{GeV}$

NC Scale is simply scaling the neutral current component by $\pm 20 \%$, which is a absolute scaling on the neutral current cross-section. The $\mathrm{NC} \operatorname{Scale}(\mathrm{E})$ is a scaling on the neutral current cross-section as a function of energy. For events with $E_{\nu}<1.5 \mathrm{GeV}$, they are scaled by $\pm 20 \%$, events with $1.5<E_{\nu}<3.0 \mathrm{GeV}$ are scaled by $\pm 10 \%$, and all other events are scaled by $\pm 5 \%$. 
The fifth parameter tests what may happen if tau neutrino appearance is not properly accounted for in the Far detector. Since tau neutrino interactions are very short in length, they will most likely be classified as neutral current events. The parameter itself simply adds tau events properly to the simulated data, but does not take taus into account with the Monte Carlo fit.

The Relative Calibration Error is a shower energy miscalibration between the Near and Far detectors. This essentially does not change the Near, but will shift the Far detector hadronic energy scale by $\pm 2 \%$. This is to study the effect of relative miscalibration between the Near and Far detectors.

The next three parameters are shower energy offsets of 50,100, and 150 $\mathrm{MeV}$. These offsets study the effect of intranuclear scattering which shifts the mean shower energy and possible reconstruction errors. It has recently been found that the shower energy offsets of 100 and $150 \mathrm{MeV}$ are an overestimate on the effect of intranuclear scattering [115]. We include them for historical reasons, and as worse case situations.

The last parameter, called SKZP which is an acronym for the authors, deals with changing the neutrino flux corrections by $\pm 1 \sigma$.

The results of the oscillation fits are shown for $f_{\text {sterile }}$ in Figure 8.14 and $\Delta m^{2}$ in Figure 8.15. The systematic parameter changed is listed to the left of each plot and the points to the right show the best fit with a black dot and the error bars are the $1 \sigma$ errors on the measurement. All fits recover the correct oscillation parameters to within $69 \%$ confidence level. Of all the parameters in this category of systematic studies, the shower energy offset has the largest impact on the oscillation parameters, although for a $50 \mathrm{MeV}$ offset the effect is not as large. 


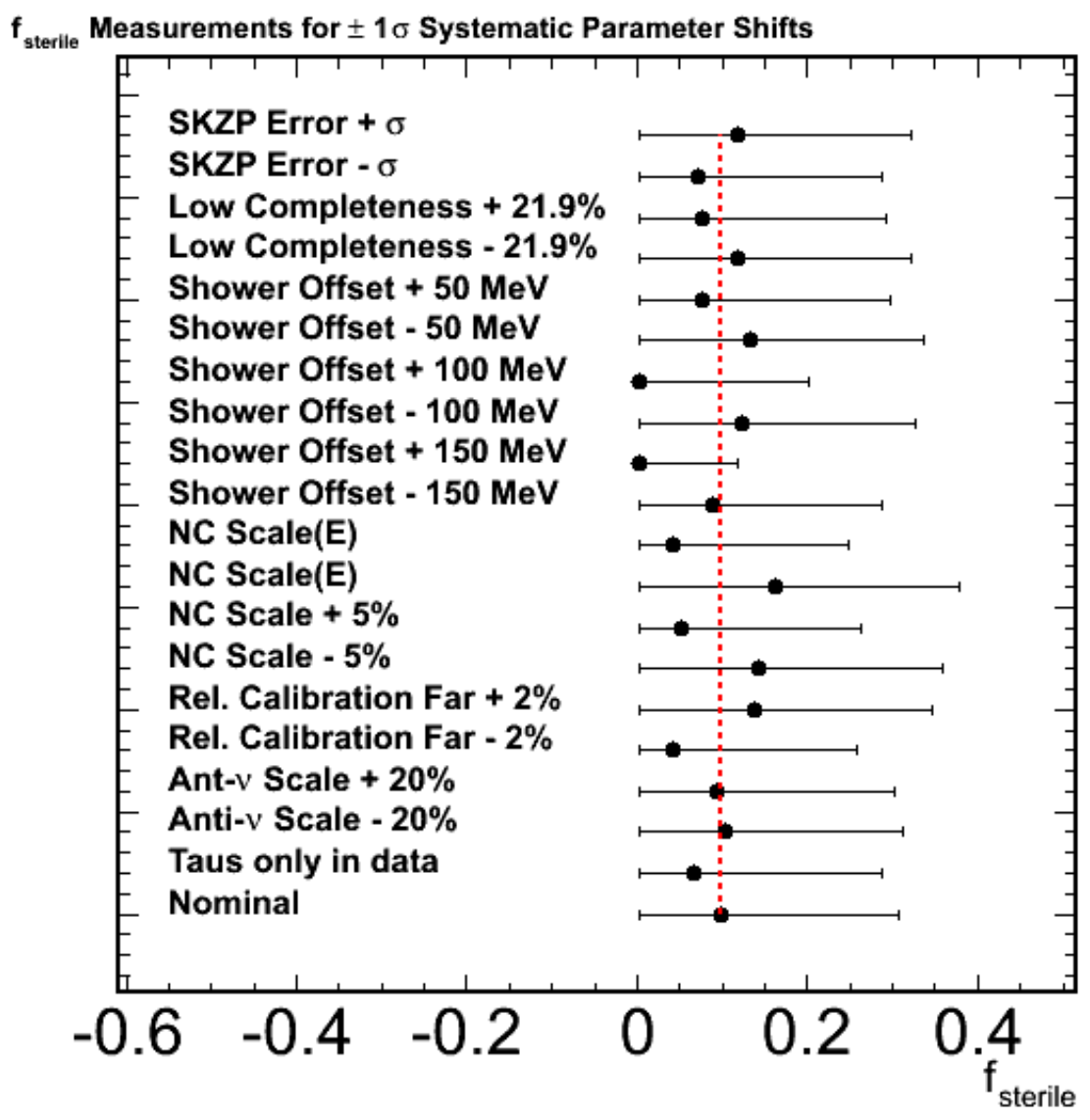

Figure 8.14: Summary of best fit values and $\pm 1 \sigma$ error on $f_{\text {sterile }}$ for systematic study III. 


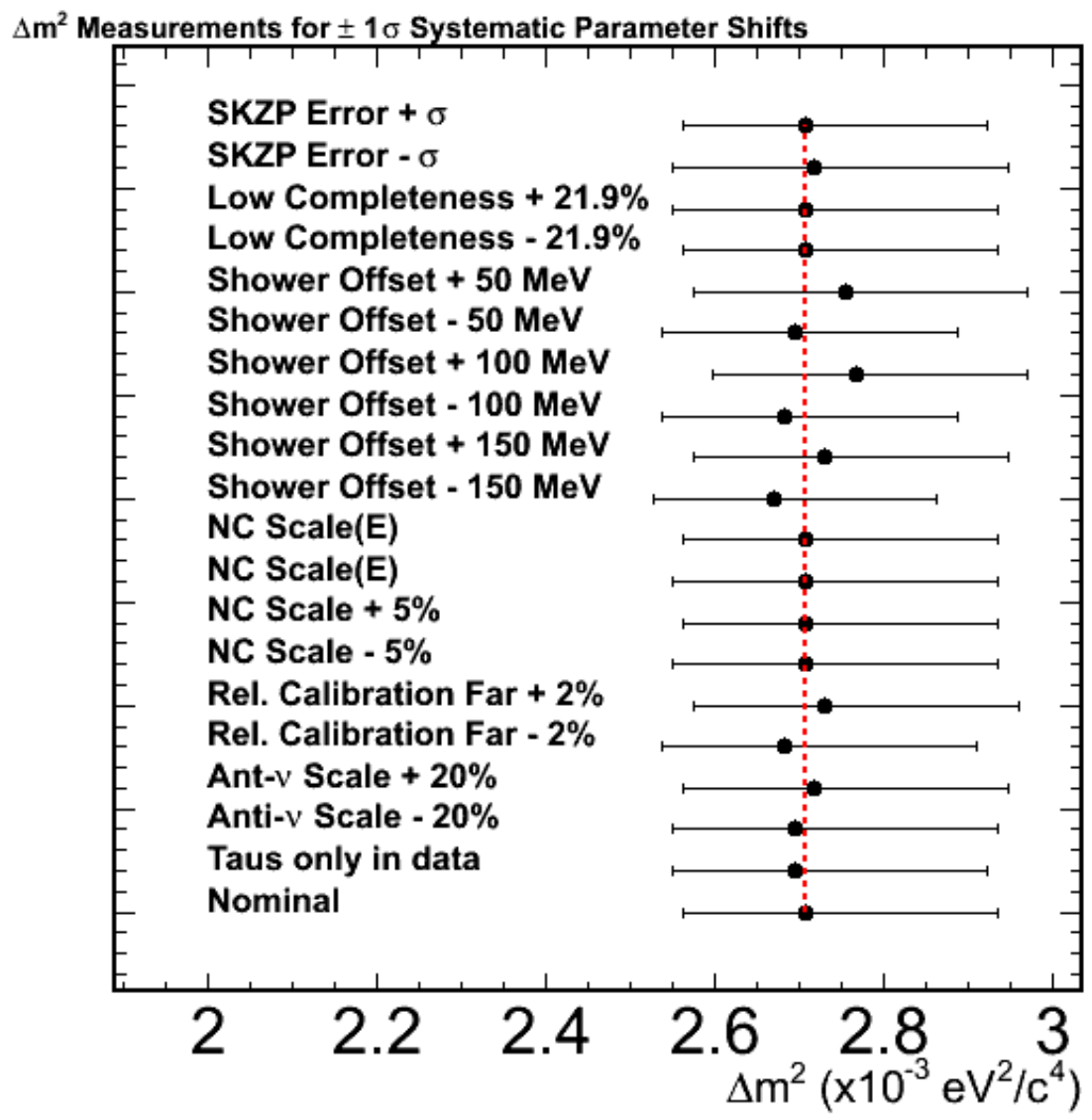

Figure 8.15: Summary of best fit values and $\pm 1 \sigma$ error on $\Delta m^{2}$ for systematic study III. 


\subsubsection{Systematic Study IV}

The fourth set of systematic studies consist of four cocktails of changes, which are just simultaneous variations of multiple parameters. This is to see how the method performs in the presence of several systematic shifts. The four cocktails are given by in Table 8.5. The first cocktail contains four parameters that happen to have very large impacts on the energy spectra from the previous three systematic studies. It is interesting to see if several large shifts will heavily bias our measurement. The second cocktail changes the anti-neutrino scaling, a kno parameter dealing with neutral current cross-section, and the overall scaling of the the neutral current cross-section as a function of energy. This is meant to study the effects of changing the cross-section for several different components of the energy spectra. The third cocktail changes only the five reconstruction parameters simultaneously that will be fit for in the Near detector. This cocktail tests how a very incomplete knowledge of our reconstruction would affect the oscillation fit. The last cocktail changes all the parameters we will be fitting for in the Near detector, except the shower and track energies. This is because the other eight are fit with the Marquardt minimization method and we wish to see if there exist any pathologies as a result of only using the Marquardt method in the Near detector fit.

The results of the oscillation fits are shown for $f_{\text {sterile }}$ in Figure 8.16 and $\Delta m^{2}$ in Figure 8.17. The systematic parameter changed is listed to the left of each plot and the points to the right show the best fit with a black dot and the error bars are the $1 \sigma$ errors on the measurement. All fits recover the correct oscillation parameters to within $69 \%$ confidence level. 


\begin{tabular}{|c|c|c|c|c|}
\hline Parameter Type & Parameter Name & Default Value & $1 \sigma$ Error & Fit Type \\
\hline \hline Simulation & CCMA & 1.0 & 0.2 & Marquardt \\
\hline Simulation & knor213223 & 1.0 & 0.2 & Marquardt \\
\hline Simulation & Shower Offset & 0.0 & $50 \mathrm{MeV}$ & Marquardt \\
\hline Calibration & Rel. Calibration Error & 1.0 & 0.02 & Marquardt \\
\hline
\end{tabular}

\begin{tabular}{|c|c|c|c|c|}
\hline Parameter Type & Parameter Name & Default Value & $1 \sigma$ Error & Fit Type \\
\hline \hline Cross-section & Anti-Neutrino & 1.0 & 0.2 & Marquardt \\
\hline Simulation & knor213223 & 1.0 & 0.2 & Marquardt \\
\hline Cross-section & NC Scale $(\mathrm{E})^{*}$ & 1.0 & $*$ & Marquardt \\
\hline
\end{tabular}

\begin{tabular}{|c|c|c|c|c|}
\hline Parameter Type & Parameter Name & Default Value & $1 \sigma$ Error & Fit Type \\
\hline \hline Reconstruction & Shower Energy & 1.0 & 0.11 & Marquardt \\
\hline Reconstruction & Track Energy & 1.0 & 0.02 & Marquardt \\
\hline Reconstruction & NC Background & 1.0 & 0.5 & Marquardt \\
\hline Reconstruction & CC Background & 1.0 & 0.05 & Marquardt \\
\hline Reconstruction & Normalization & 1.0 & 0.03 & Marquardt \\
\hline
\end{tabular}

\begin{tabular}{|c|c|c|c|c|}
\hline Parameter Type & Parameter Name & Default Value & $1 \sigma$ Error & Fit Type \\
\hline \hline Simulation & knor112122** & 1.0 & $1.0,0.33$ & Marquardt \\
\hline Simulation & knor113123** & 1.0 & $1.0,0.33$ & Marquardt \\
\hline Simulation & knor212222 & 1.0 & 0.2 & Marquardt \\
\hline Simulation & knor213223 & 1.0 & 0.2 & Marquardt \\
\hline Reconstruction & Normalization & 1.0 & 0.03 & Marquardt \\
\hline Simulation & CCMA & 1.0 & 0.15 & Marquardt \\
\hline Reconstruction & NC Background & 1.0 & 0.5 & Marquardt \\
\hline Reconstruction & CC Background & 1.0 & 0.05 & Marquardt \\
\hline
\end{tabular}

Table 8.5: Systematic Parameters Shifted in Cocktails I, II, III, and IV respectively. $*=20 \%$ for $\mathrm{E}<1.5 \mathrm{GeV}, 10 \%$ for $1.5<\mathrm{E}<3.0 \mathrm{GeV}$, and $5 \%$ for $\mathrm{E}>3.0 \mathrm{GeV}$. ** $=$ knor112122 and knor113123 consist of two parameters with two different errors. knor112 and knor113 have an error of 100\%, knor122 and knor123 have an error of $33 \%$. 


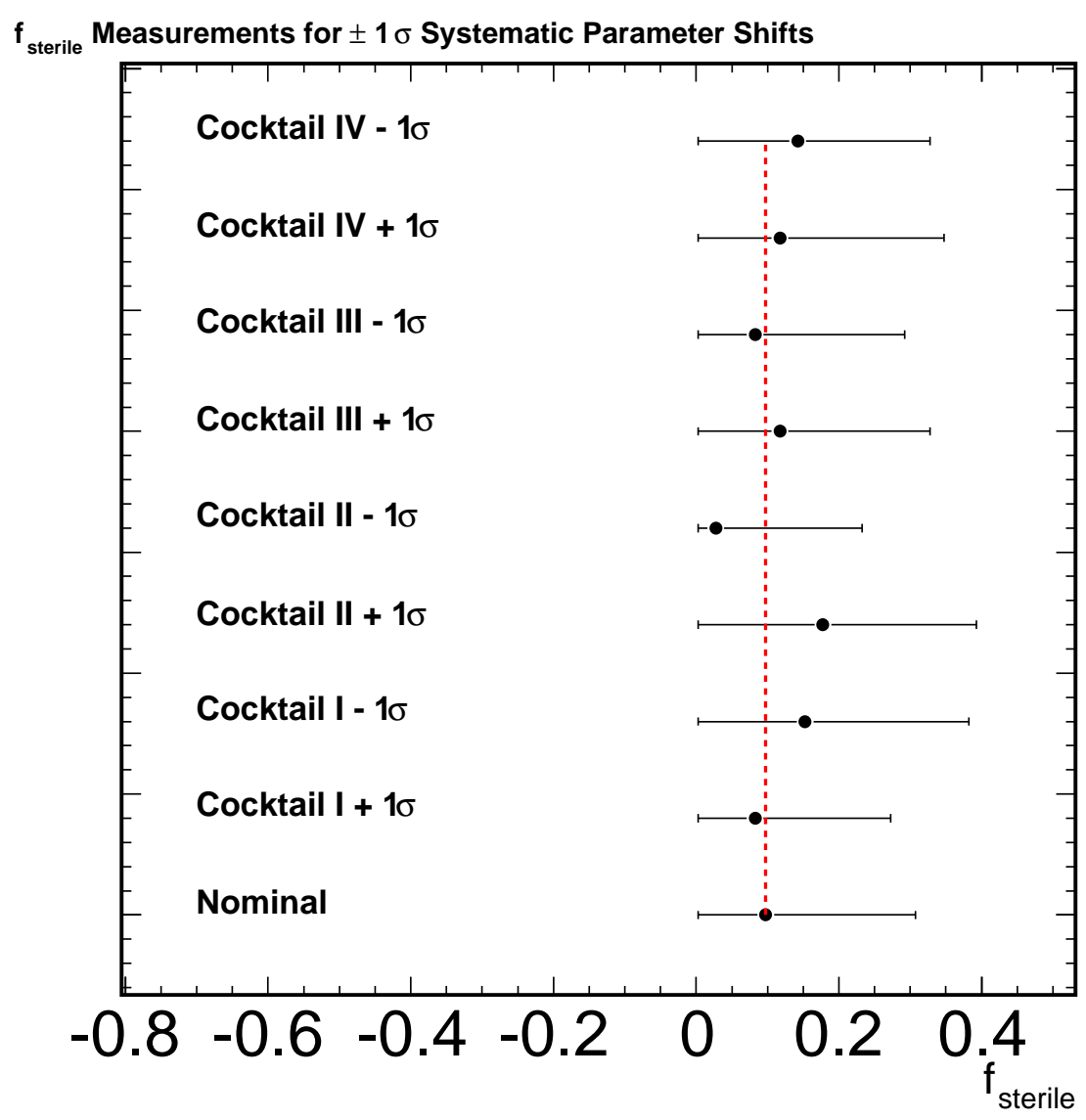

Figure 8.16: Summary of best fit values and $\pm 1 \sigma$ error on $f_{\text {sterile }}$ for systematic study IV. 


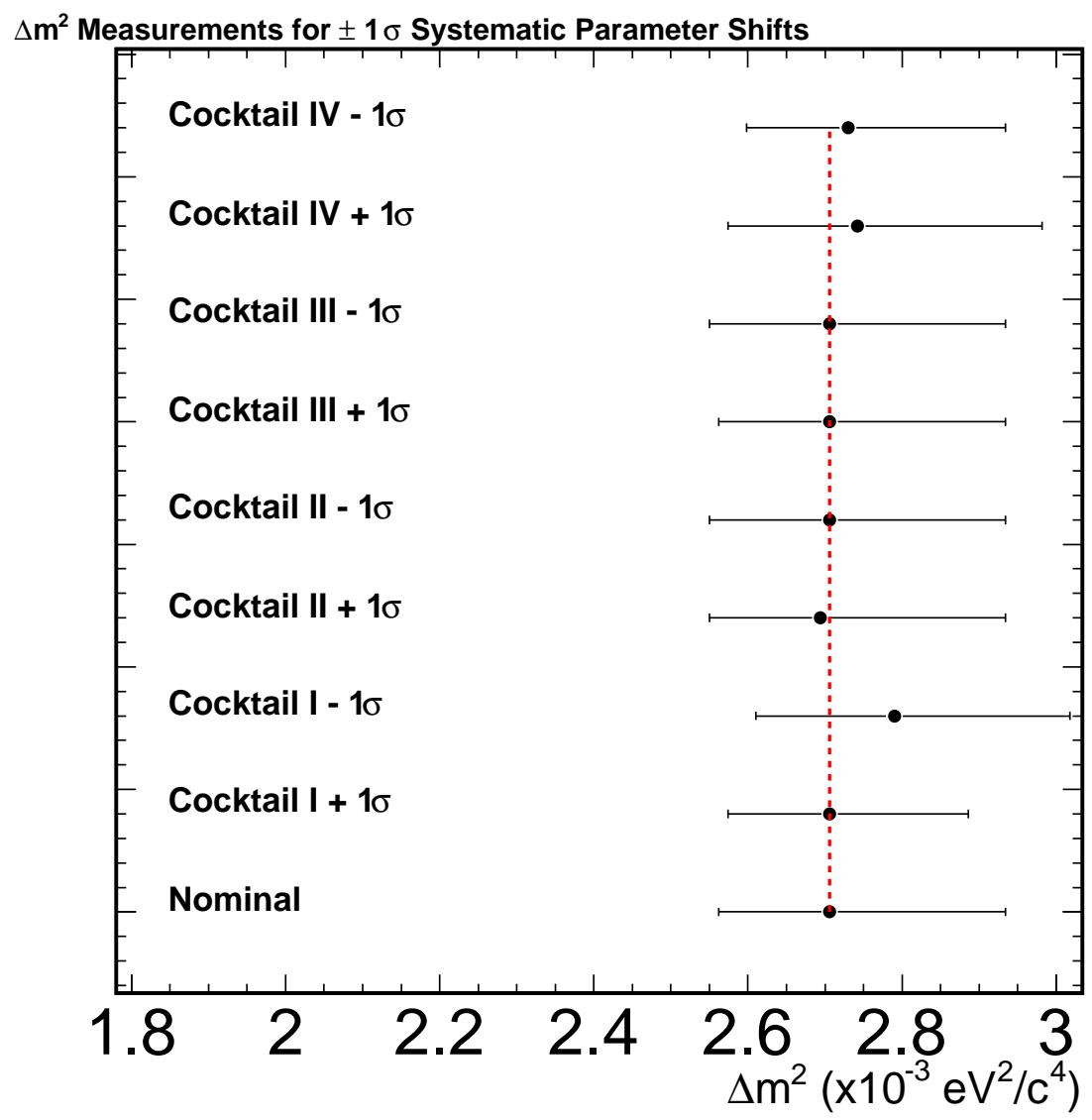

Figure 8.17: Summary of best fit values and $\pm 1 \sigma$ error on $\Delta m^{2}$ for systematic study IV. 


\subsubsection{Systematic Study V}

The fifth set of systematic studies consists of changing the parameters fit for in the Near detector by $\pm 1 \sigma$ for the Far detector simulated data and we ignore the Near detector. This is essentially a $\pm 1 \sigma$ change in the Far Detector and then a subsequent fit for oscillation parameters. This is the worst case scenario since there is no information from the Near detector to correct our Far detector Monte Carlo. This is expected to give the largest shifts from the input oscillation parameters.

The results of the oscillation fits are shown for $f_{\text {sterile }}$ in Figure 8.18 and $\Delta m^{2}$ in Figure 8.19. The systematic parameter changed is listed to the left of each plot and the points to the right show the best fit with a black dot and the error bars are the $1 \sigma$ errors on the measurement. All fits recover the correct oscillation parameters to within $69 \%$ confidence level. Of all the parameters in this category of systematic studies, the simulation parameter CCMA and the hadronic energy scale have the largest impacts and only in the case of CCMA do we not recover the true oscillation parameters to the $69 \%$ confidence level. 


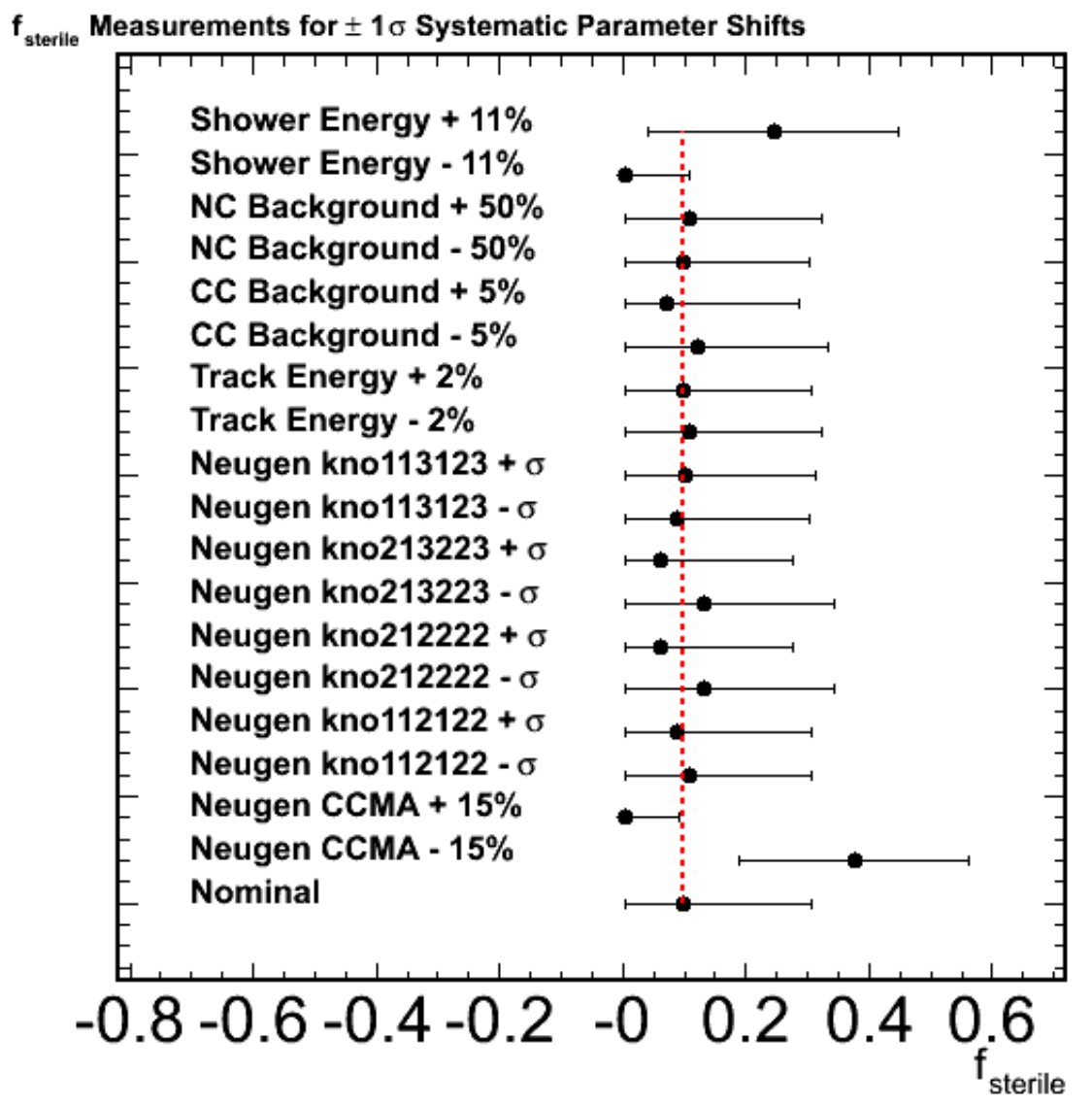

Figure 8.18: Summary of best fit values and $\pm 1 \sigma$ error on $f_{\text {sterile }}$ for systematic study V. 


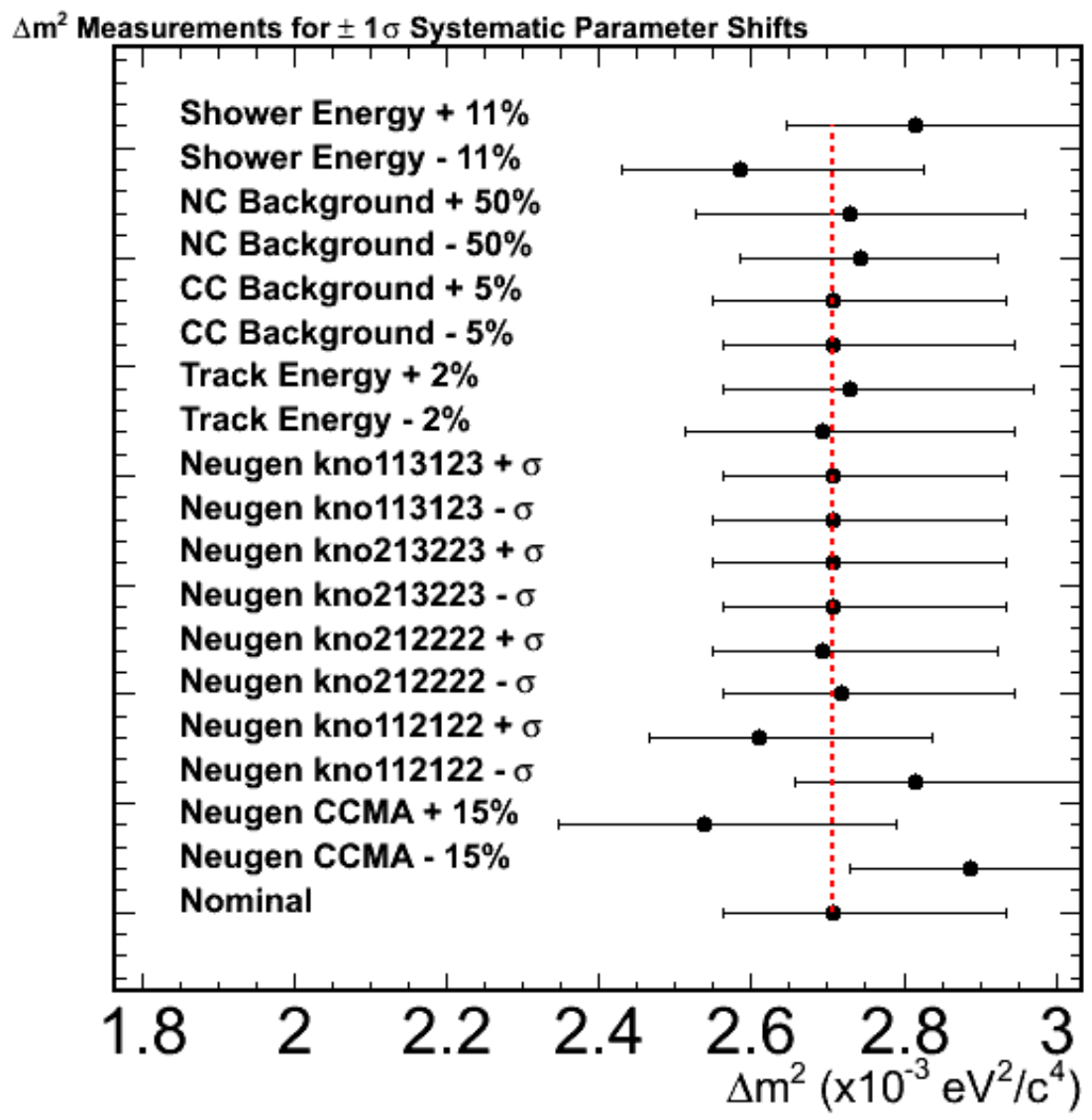

Figure 8.19: Summary of best fit values and $\pm 1 \sigma$ error on $\Delta m^{2}$ for systematic study V. 


\subsection{Summary of Systematic Studies}

The last several subsections have described the results of five different systematic studies. The results of all these tests show that the Marquardt-style NDFit can withstand any biases from an imperfect knowledge of many systematic parameters. The only fit that did not properly measure the true oscillation parameters to $69 \%$ confidence level was when CCMA was changed by $\pm 1 \sigma$ in the Far detector without information from the Near detector. This is not an issue, since information is obtained from the Near detector to control this effect.

The next section describes the results of fitting an independent simulated data set in the Far detector. The input oscillation parameters were hidden in order to test fitting routines without any bias.

\subsection{Mock Data Challenge}

Systematic studies are extremely useful for understanding the effect of unknown information on our oscillation measurements. Individual proponents of different extrapolation techniques can test their analysis chain very rigorously with these types of studies. In order to provide a test that is independent of these systematic studies, individuals take part in a Mock Data Challenge (MDC). The MDC is a generated set of Far detector data with unknown oscillation parameters applied and hidden until the various extrapolations can produce their results. This allows the collaboration to verify the readiness of the different analysis groups, and also helps to uncover issues that require more effort.

The results of two MDC sets are shown in the following two sections. The separate MDC sets consist of 100 individual files, each consisting of $2.5 \times 10^{20}$ protons on target, which is approximately the exposure used for the final results in this work. This gives a total of $2.5 \times 10^{22}$ protons on target for each MDC. Given 
such a high statistics sample, statistical fluctuations should be very small.

\subsubsection{Mock Data Challenge Set I Results}

The first Mock Data Challenge set had input parameters of $\Delta m^{2}=0.00273 \mathrm{eV}^{2} / \mathrm{c}^{4}$, $\sin ^{2}(2 \theta)=0.9925$, and $f_{\text {sterile }}=0.0$. The best fit parameters were $\Delta m^{2}=0.00275$ $\mathrm{eV}^{2} / \mathrm{c}^{4}, \sin ^{2}(2 \theta)=0.975$, and $f_{\text {sterile }}=0.025$. The correct oscillation parameters were recovered within $90 \%$ confidence level. The energy spectra of the total MDC set in the Far detector is shown in Figure 8.20. The top left plot is the neutral current energy spectra with the black dots being the mock data, the blue is the nominal monte carlo, and the red is the best oscillation fit. The top right plots the same for charged current events. The bottom two plots show the ratios of data to nominal Monte Carlo in blue and data to fit Monte Carlo in red. The two dimensional $\Delta m^{2}$ versus $f_{\text {sterile }}$ contour is shown in Figure 8.21 with the $69 \%, 90 \%$, and $99 \%$ confidence level contours overlaid in addition to the one dimensional contour for $f_{\text {sterile. }}$

Of interest is the results of fitting each of the 100 individual $2.5 \times 10^{20}$ subruns to see what statistical fluctuations can do to our fit. We do this for all the subruns and histogram the best fit results of each of the fits in Figure 8.22. The top left plot shows the best fit points for $f_{\text {sterile, }}$, the top middle plot shows the best fit points for $\sin ^{2}(2 \theta)$ and the top right is the best fit points for $\Delta m^{2}$. The bottom plots show the best fit point and it's $1 \sigma$ error versus subrun number. The red lines superimposed on the bottom plots are the best fit from the full $2.5 \times 10^{22}$ protons on target fit. With lower statistics, the plot indicates that we could see a substantial fluctuation in our measurement of $f_{\text {sterile }}$. 

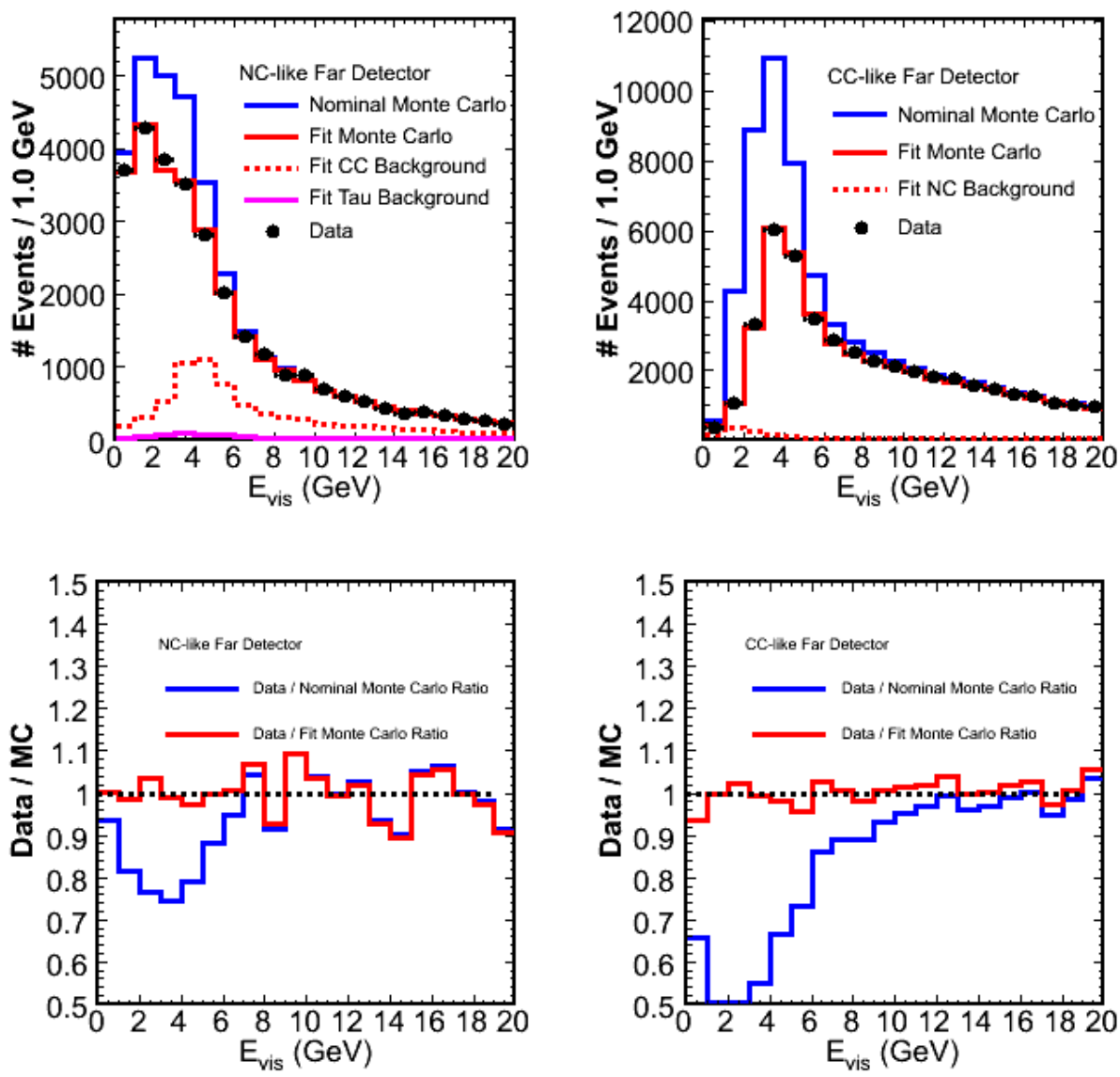

Figure 8.20: The top left plot is the neutral current energy spectrum and the top right is the charged current for the first MDC set. The black dots are the mock data and the blue spectrum is the nominal Monte Carlo, followed by the best fit in red. The bottom two plots are the ratios of mock data to nominal Monte Carlo in blue and mock data to best fit Monte Carlo in red. 

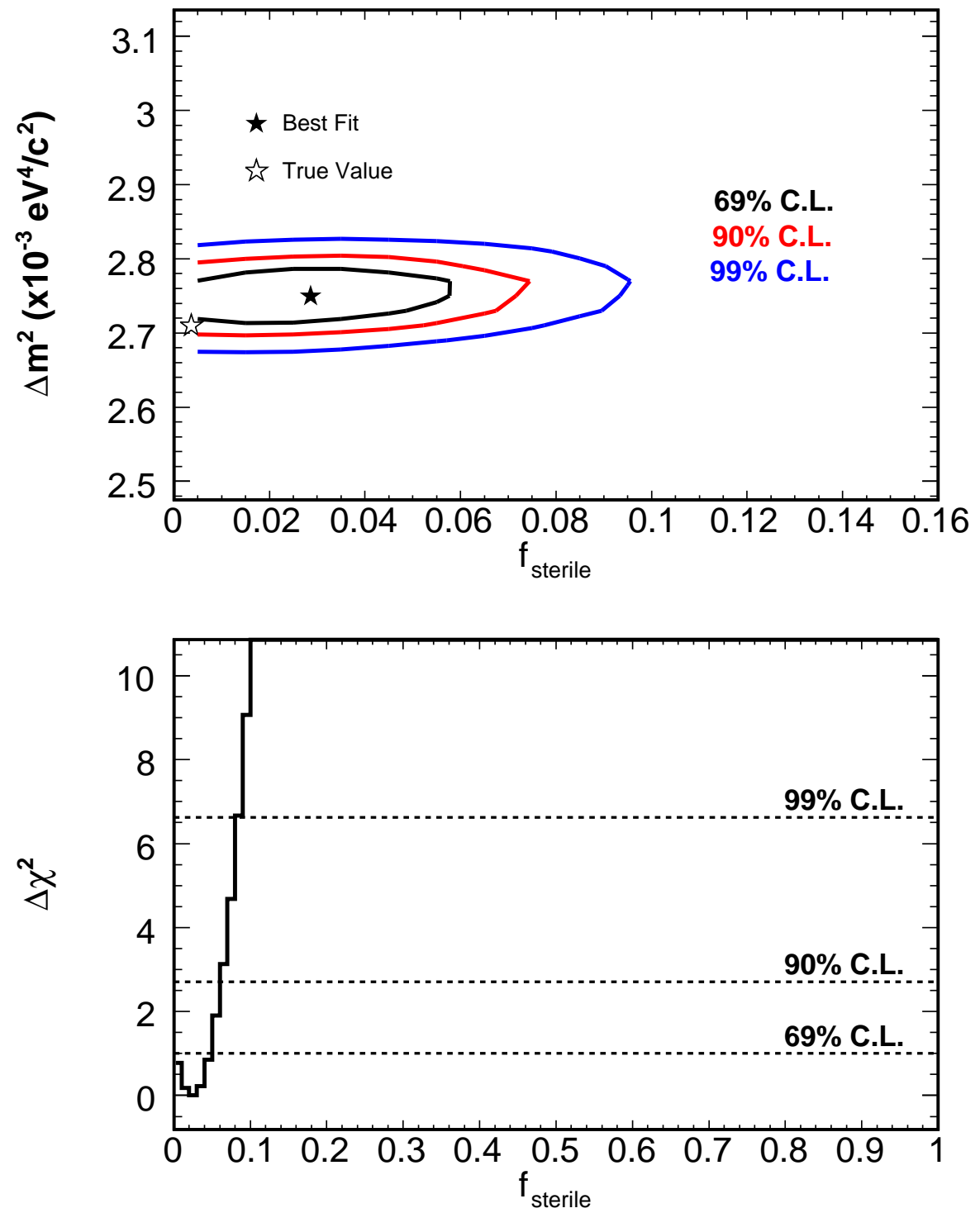

Figure 8.21: Oscillation results from the first MDC set. The top plot is the 2-D contour for $f_{\text {sterile }}$ vs $\Delta m^{2}$ with $69 \%, 90 \%$, and $99 \%$ confidence levels superimposed. The bottom plot is the 1-D contour for $f_{\text {sterile }}$ 

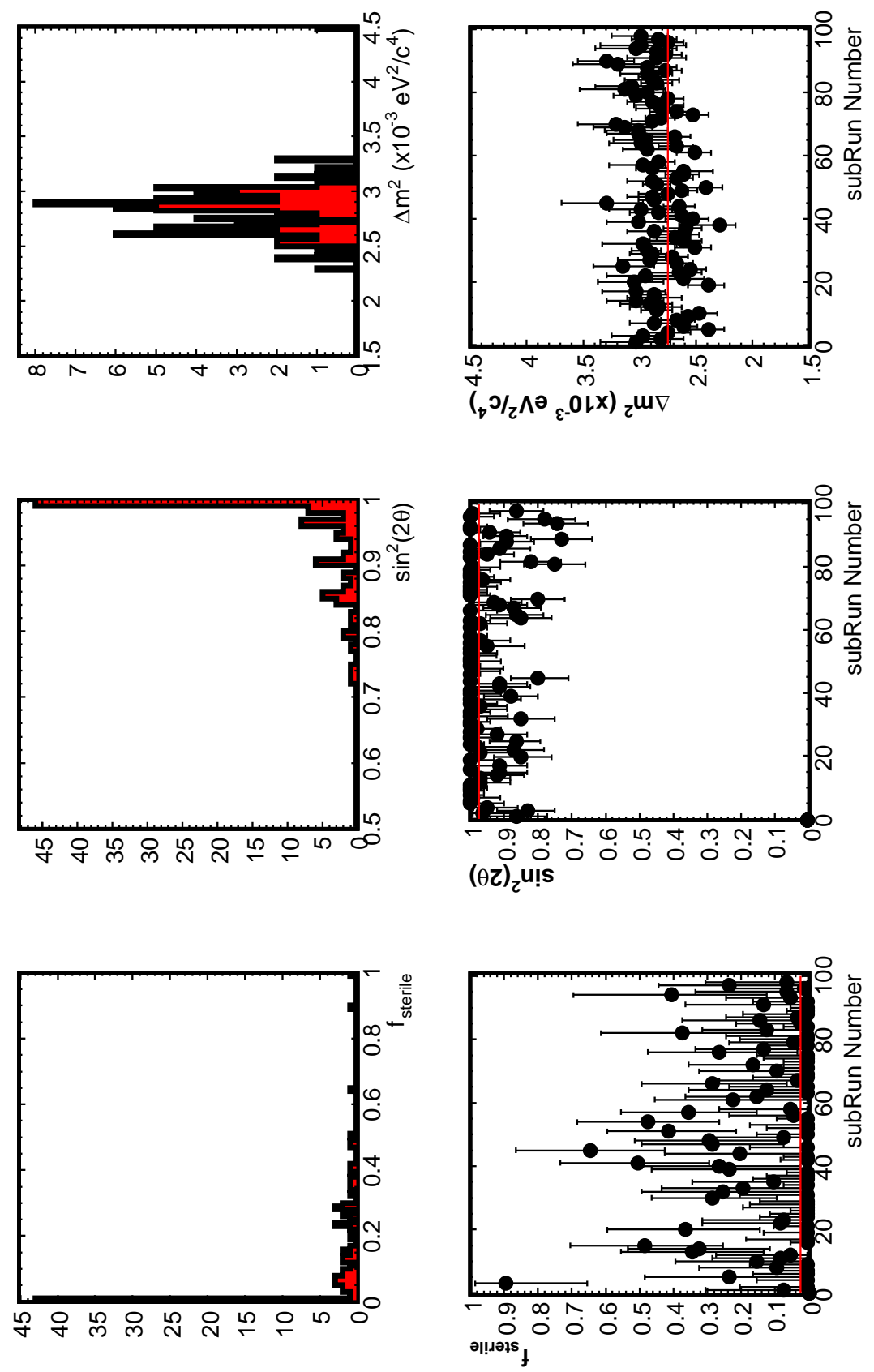

Figure 8.22: Summary of results as a function of individual $2.5 \times 10^{20}$ protons on target subruns from the first MDC set. 


\subsubsection{Mock Data Challenge Set II Results}

The second Mock Data Challenge set had input parameters of $\Delta m^{2}=0.00378 \mathrm{eV}^{2} / \mathrm{c}^{4}$, $\sin ^{2}(2 \theta)=0.758$, and $f_{\text {sterile }}=0.25$. The best fit parameters were $\Delta m^{2}=0.00373 \mathrm{eV}^{2} / \mathrm{c}^{4}$, $\sin ^{2}(2 \theta)=0.775$, and $f_{\text {sterile }}=0.245$. The correct oscillation parameters were recovered within $69 \%$ confidence level. The energy spectra of the total MDC set is shown in Figure 8.23. The top left plot is the neutral current energy spectra with the black dots being the mock data, the blue is the nominal Monte Carlo, and the red is the best oscillation fit. The top right plots is the same for charged current events. The bottom two plots show the ratios of data to nominal Monte Carlo in blue and data to fit Monte Carlo in red. The two dimensional $\Delta m^{2}$ vs $f_{\text {sterile }}$ contour is shown in Figure 8.24 with the $69 \%, 90 \%$, and $99 \%$ confidence level contours overlaid in addition to the one dimensional contour for $f_{\text {sterile }}$.

As for the first MDC set, we show in Figure 8.25 the the results of fitting 58 individual $2.5 \times 10^{20}$ subruns to see what statistical fluctuations can do to our fit. The plot has the same format as we described for Figure 8.22. 

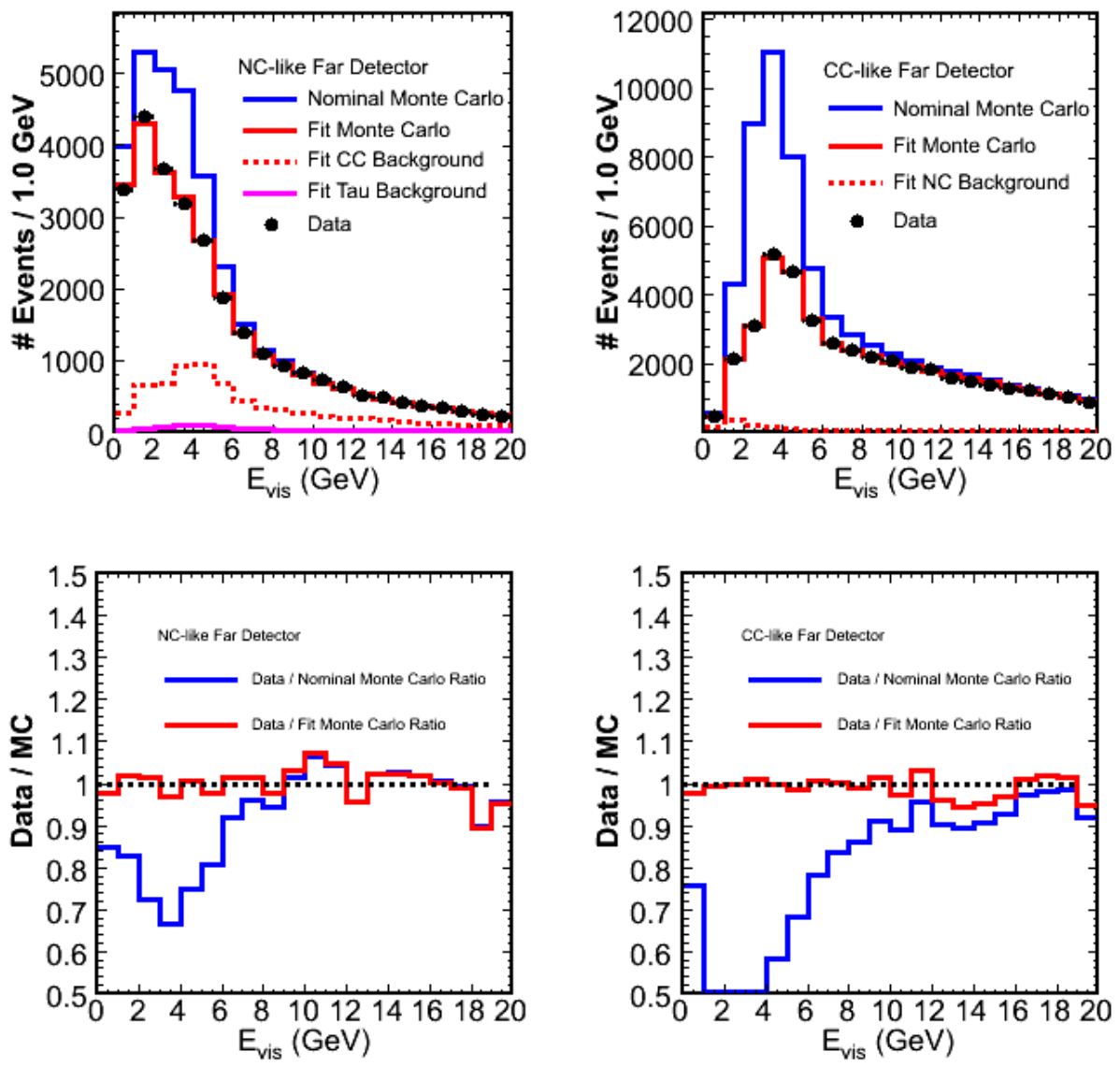

Figure 8.23: The top left plot is the neutral current energy spectrum and the top right is the charged current for the second MDC set. The black dots are the mock data and the blue spectrum is the nominal Monte Carlo, followed by the best fit in red. The bottom two plots are the ratios of mock data to nominal Monte Carlo in blue and mock data to best fit Monte Carlo in red. 

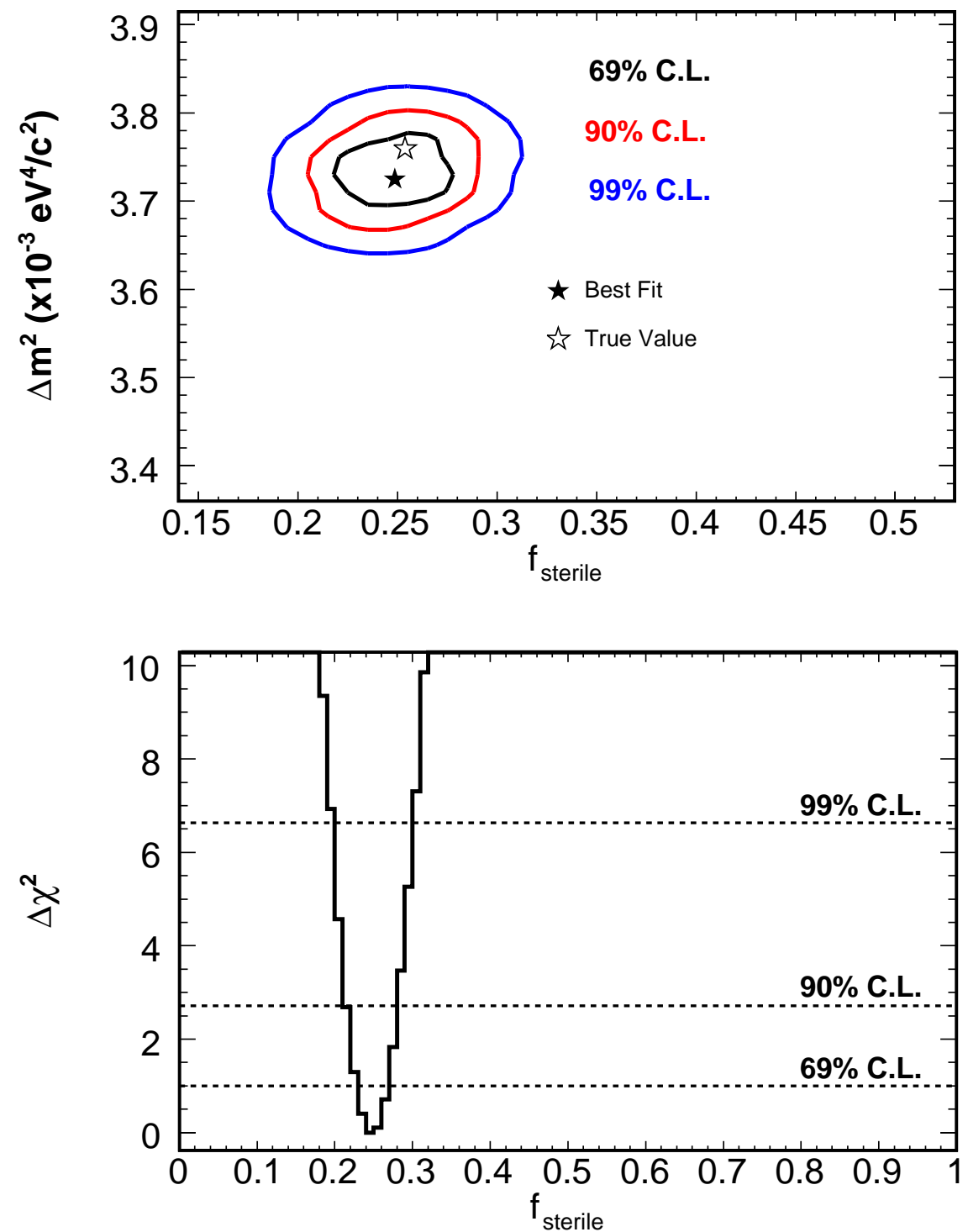

Figure 8.24: Oscillation results from the second MDC set. The top plot is the 2-D contour for $f_{\text {sterile }}$ vs $\Delta m^{2}$ with $69 \%, 90 \%$, and $99 \%$ confidence levels superimposed. The bottom plot is the 1-D contour for $f_{\text {sterile }}$ 

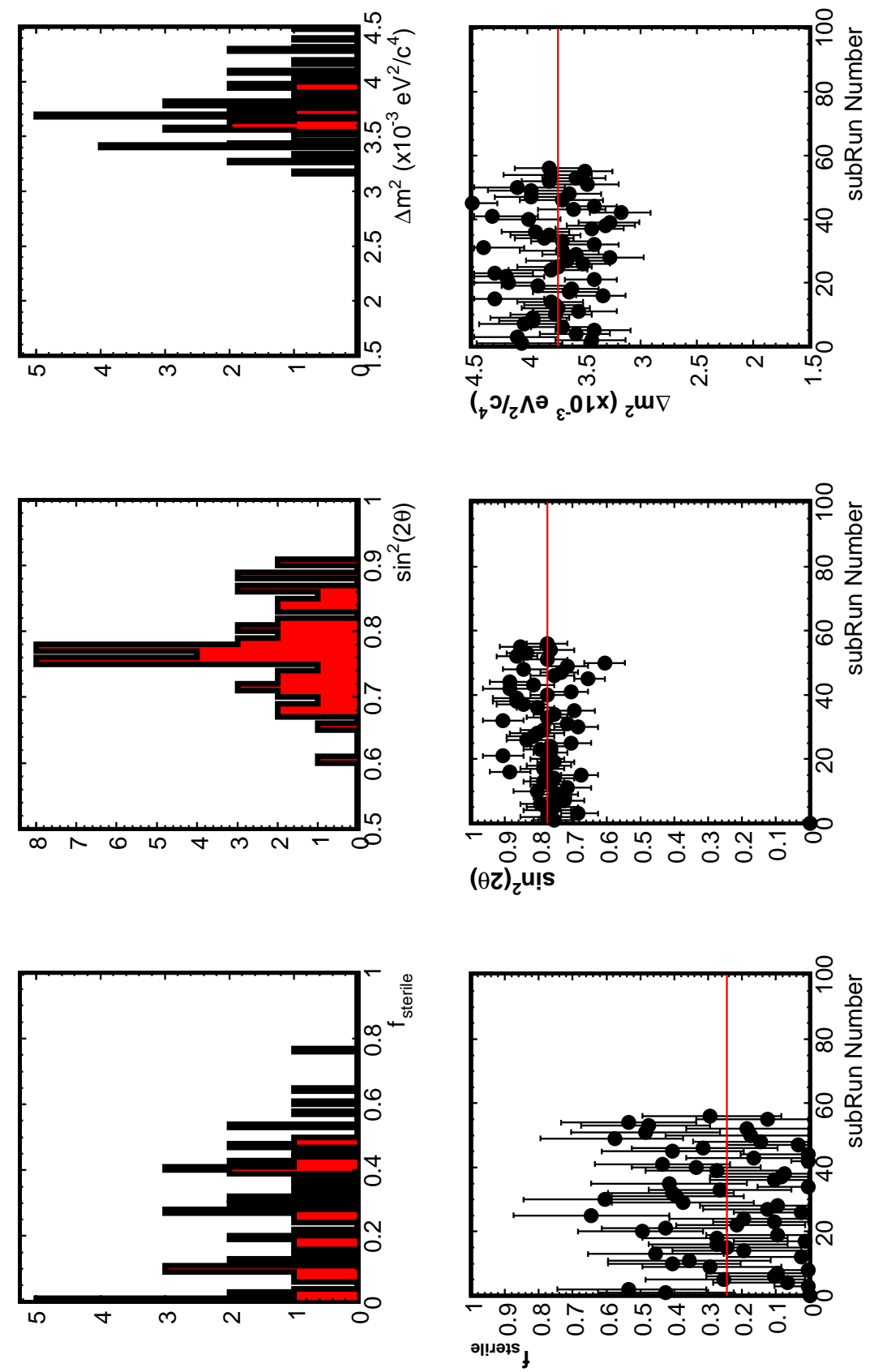

Figure 8.25: Summary of results as a function of individual $2.5 \times 10^{20}$ protons on target subruns from the second MDC set. 


\subsection{Conclusions}

The Marquardt-style NDFit method has been described in detail. An overview of the systematic parameters that will be fit in the Near detector have been presented and our rational for choosing them. The robustness of the Marquardt-style NDFit has been demonstrated through five different systematic studies in addition to successfully completing the Mock Data Challenge. The next chapter will report the results of a search for sterile neutrinos in MINOS. 


\section{Chapter 9}

\section{$\nu_{\mu} \rightarrow \nu_{\text {sterile Oscillation Search }}$}

\subsection{Introduction}

Having developed our selection criteria and data quality checks for both the Near and Far detectors, in addition to rigorous reconstruction and systematic studies, the analysis is now in a position to proceed with the $\nu_{\mu} \rightarrow \nu_{\text {sterile }}$ oscillation search. The Far detector data set will be described along with plots to cross-check our selection. This is followed by the Near detector fit and the result for the Far detector Monte Carlo charged current and neutral current energy spectrum prediction. Far detector data events are then selected and the oscillation fit is performed. The chapter is ended with the parameter space contours for the oscillation measurement and conclusions.

\subsection{Data Set and Cross-checks}

The Far detector data set is split into two different run periods known as Run I and Run II. Shortly after the comparison between Run I and Run II data, a noticeable difference for charged current energy spectra in the nominal beam configuration between the two run periods was observed. It was determined through Fermilab 


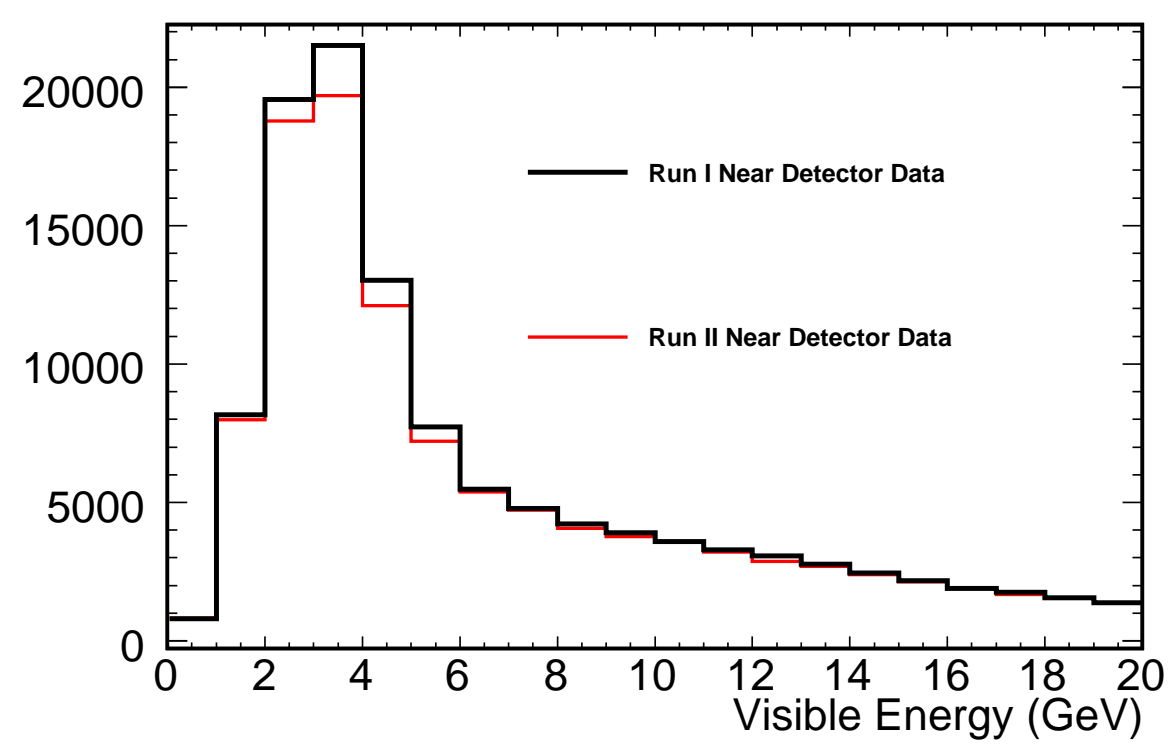

Figure 9.1: Run I and Run II shutdown Near detector charged current energy spectra.

accelerator personal and beam systematics simulations that the most likely cause was a misalignment in the target position consistent with the target position being at $-9 \mathrm{~cm}$ for Run II data as appose to the nominal $-10 \mathrm{~cm}$ [117]. Figure 9.1 illustrates this difference.

A solution [117] was proposed so that it was still possible to combine Far detector data from both run periods but the fraction of the Far detector Monte Carlo corresponding to the Run II data would have a correction associated with it to account for this $1 \mathrm{~cm}$ difference in target position.

Throughout this chapter we will apply this correction where needed without necessarily elaborating since the correction will be transparent to the analysis. 


\subsubsection{Quantities as a function of time}

The Run I phase started in May 2005 and ran until February 2006 with a total exposure of $1.245 \times 10^{20}$ protons on target. The Run II phase started after the annual Fermilab summer accelerator shutdown in September 2006 and ran until March 2007 with a exposure of $1.225 \times 10^{20}$ protons on target. These two run periods correspond to MINOS runs 31720 - 33791 and 36570 - 37832 for Runs I and II respectively.

In order to validate the stability and accumulation of neutrino interactions in the Far detector, Figure 9.2 shows the number of protons on target and number of selected neutrino beam events as a function of time for three samples of the data. The three samples consist of solely CC-like, NC-like, and all events combined. These figures show the variations of accumulated protons and events follow each other.

To have a understanding of this accumulation versus time, Figure 9.3 plots the integrated protons on target and integrated number of CC-like, NC-like, and all events as a function of time. The correlation between accumulated protons on target and events is very apparent. 


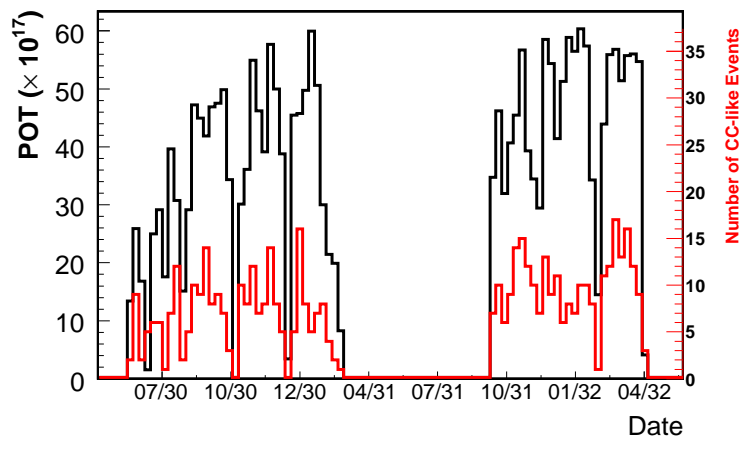

(a)

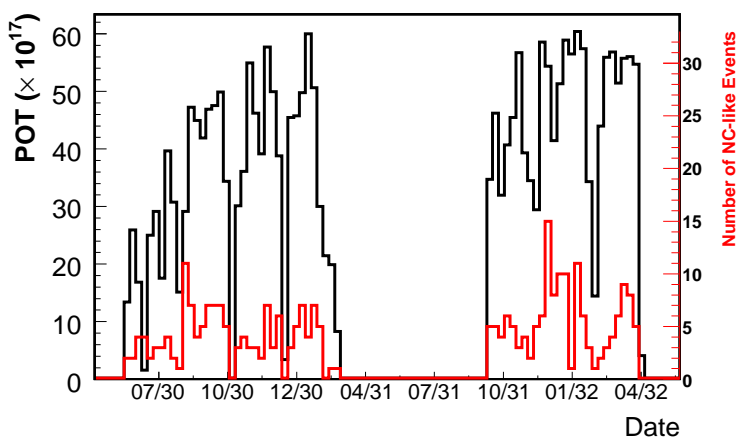

(b)

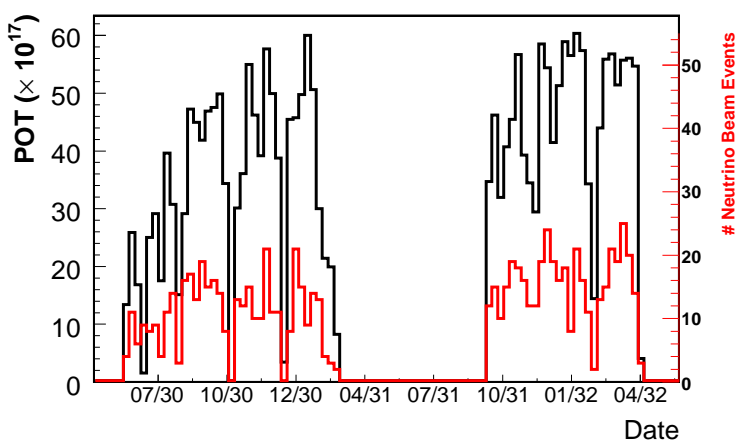

(c)

Figure 9.2: The number of protons on target and neutrino beam interactions as a function of time for (a) CC-like events (b) NC-like events (c) CC-like combined with NC-like. 


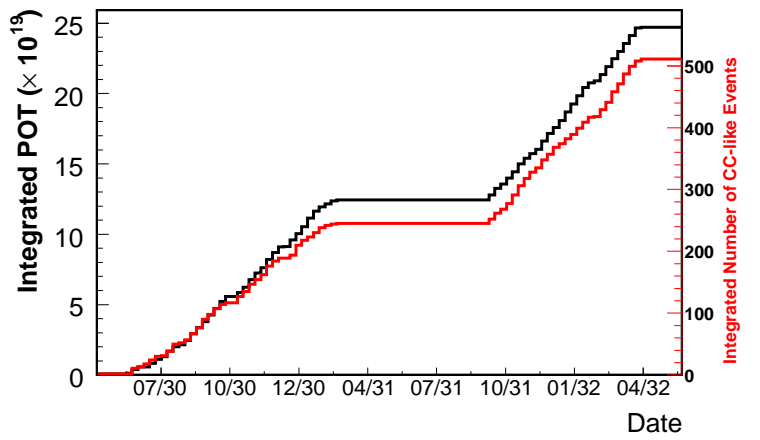

(a)

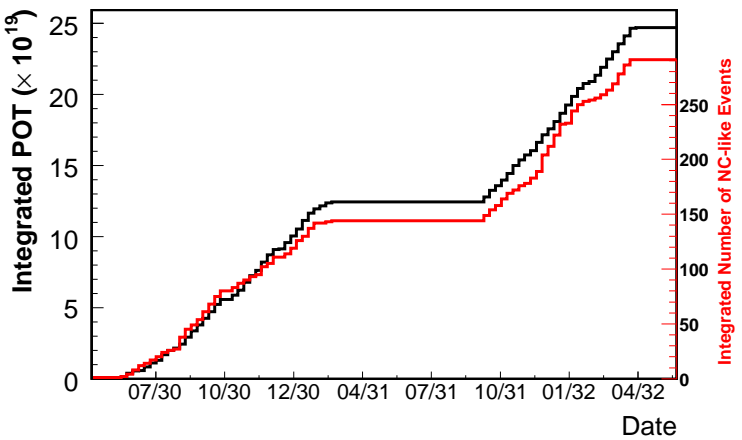

(b)

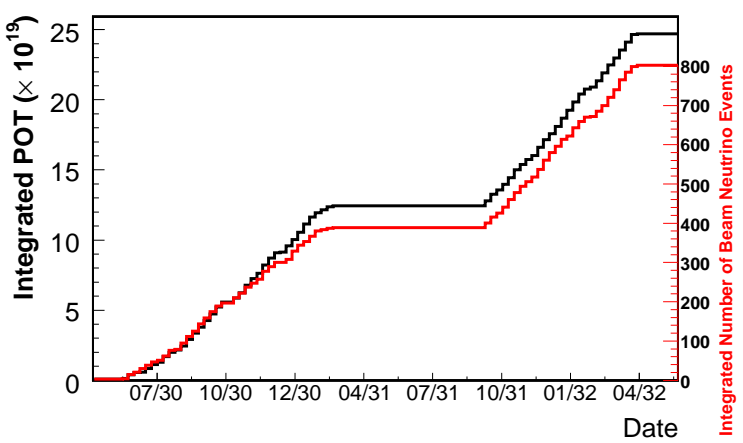

(c)

Figure 9.3: The integrated protons on target and selected neutrino interactions for (a) CC-like events (b) NC-like events (c) CC-like combined with NC-like. 


\subsubsection{Timing}

As described in Chapter 7, all beam neutrinos that interact in the Far detector should be correlated with the time they leave Fermilab. Since the length of the beam spill is $10 \mu \mathrm{s}$, one expects all beam neutrinos to be found in a $10 \mu \mathrm{s}$ window around the prediction of their arrival from Fermilab. Figure 9.4 shows the time of arrival with respect to the Fermilab prediction for CC-like, NC-like, and all selected neutrino events in the Far detector. All events are situated in a $10 \mu \mathrm{s}$ window as expected and we can be assured that true beam neutrinos are indeed selected. An example of a CC-like and NC-like neutrino beam candidate is shown in Figure 9.5

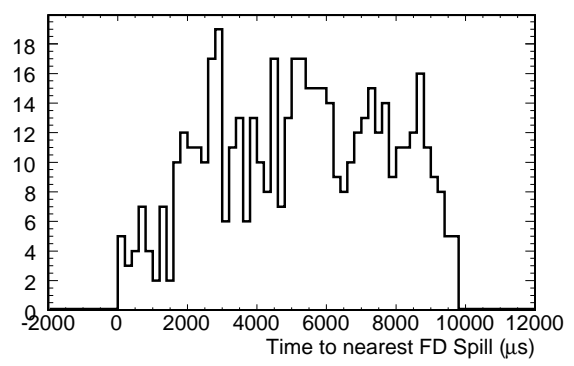

(a)

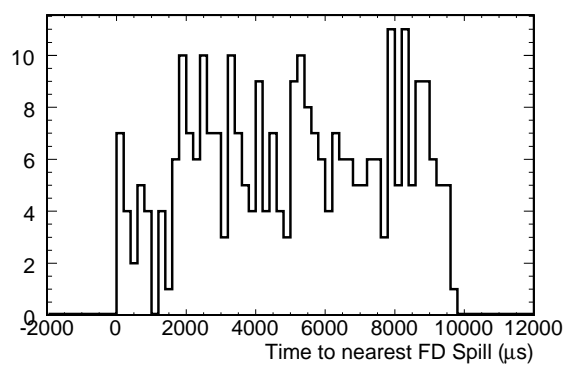

(b)

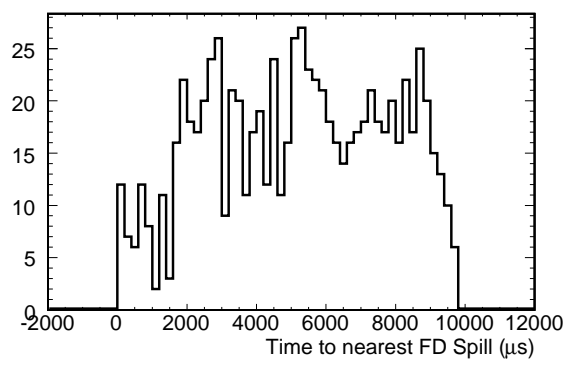

(c)

Figure 9.4: The time of the neutrino interaction with respect to the timing prediction from Fermilab for (a) CC-like events (b) NC-like events (c) CC-like combined with NC-like 

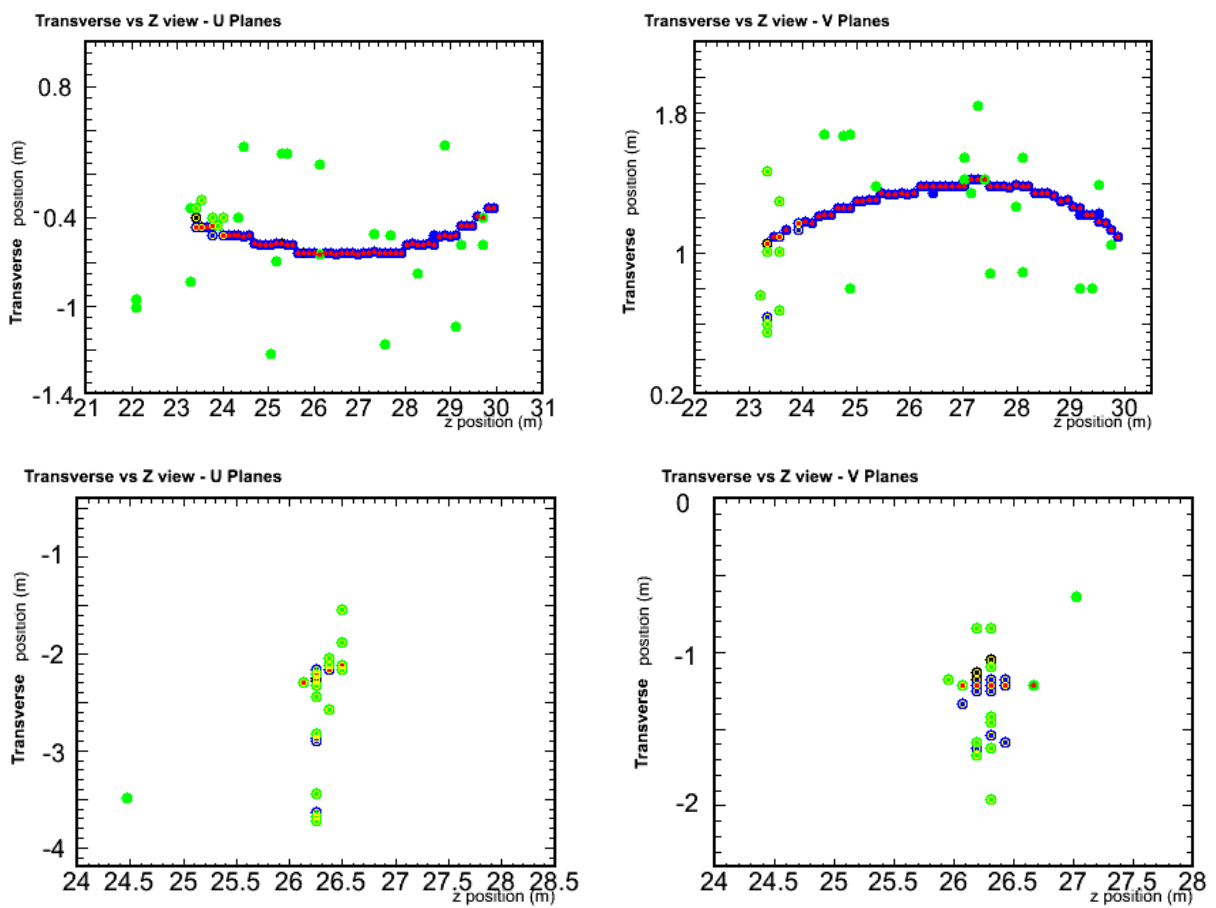

Figure 9.5: Two examples of a typical charged current neutrino beam candidate in the top plot and a neutral current event in the bottom. The axis are transverse versus longitudinal position in the Far detector.

\subsubsection{Fiducial Volume Distributions}

Another cross-check that can be performed is to verify that all the event vertices for the selected neutrino events are within our fiducial volume, in addition to being evenly distributed. Plotted in Figure 9.6 are the longitudinal vertices for CC-like, NC-like, and all events, and the $(x, y)$ vertices in Figure 9.7. 


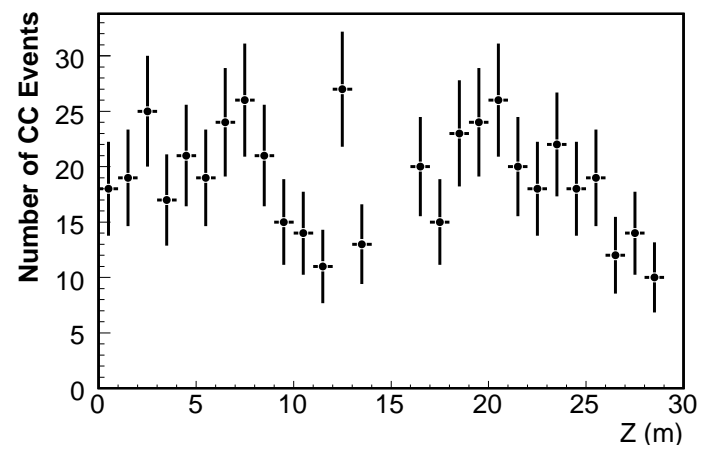

(a)

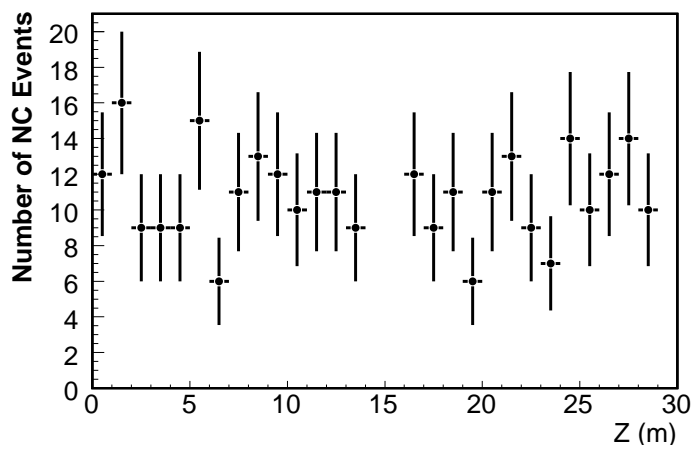

(b)

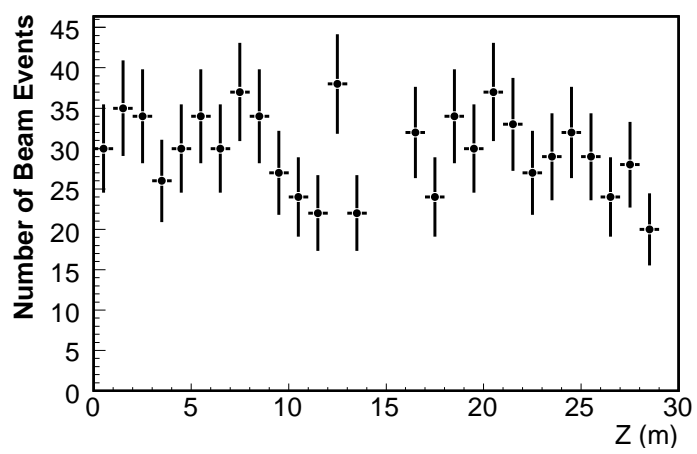

(c)

Figure 9.6: Longitudinal event vertices for (a) CC-like events (b) NC-like events (c) CC-like combined with NC-like. 


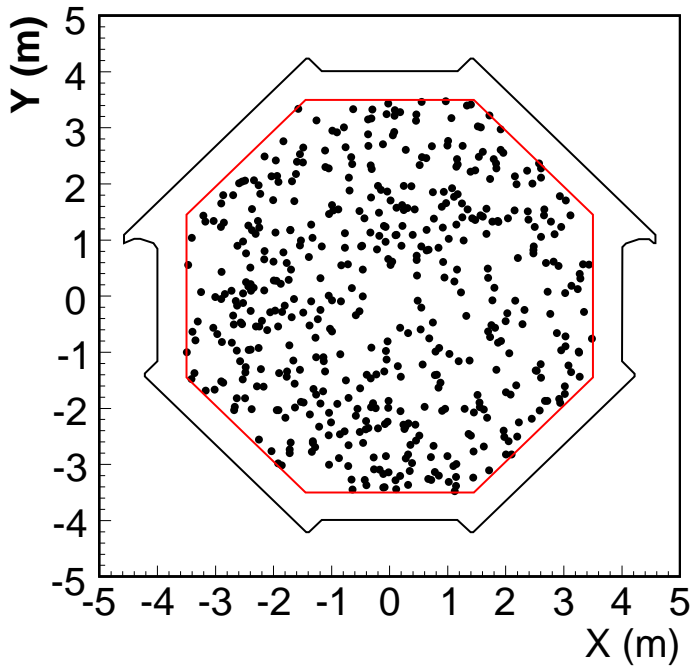

(a)

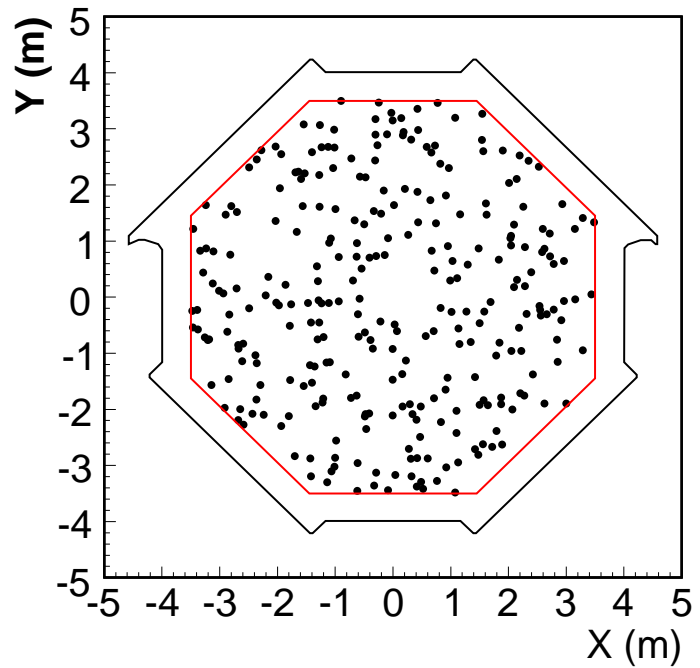

(b)

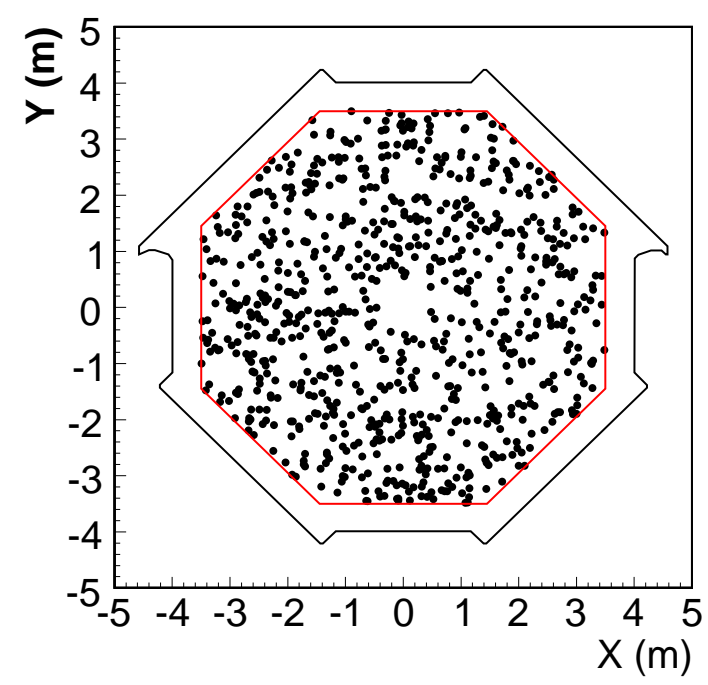

(c)

Figure 9.7: $(x, y)$ vertex in the Far detector for (a) CC-like (b) NC-like (c) CC-like combined with NC-like. 


\subsection{Near Detector Fit and Far Detector Prediction}

Since all the selected neutrino events occur within the time window allowed by the Fermilab time prediction, events are accumulating as expected versus time, and the vertices are properly distributed in our detector, we proceed with the oscillation analysis by making a prediction for the Far detector energy spectra.

Figure 9.8 shows the CC-like and NC-like energy spectra in the Near detector. The black dots are the Near detector data, the blue spectrum is the nominal Monte Carlo, and the red spectrum is the best fit to the data as performed by the Marquardt-Style fitting for ten parameters in Table 8.3. The plots show that the fit is pulling the Monte Carlo in the direction of the data and the specific results are tabulated in Table 9.1.

Examining Table 9.1, it is immediately noticed that the the shower and track energies are pulled toward smaller values. The shower energy is shifted by $-3 \%$ and the track energy is shifted by $-4.6 \%$. These changes are not necessarily indicative of a miscalibration in the shower and track reconstructed energies. These two parameters happen to have very powerful effects if changed on the energy spectrum. The fit therefore pulls them very hard because they can substantially improve agreement between data and Monte Carlo. The ideal result from the Near Detector fit is to accurately measure the physics parameters that results in data and Monte Carlo differences. One must take the results of the Near detector fit not as a actual measurement of each individual parameter but simply the best attempt to resolve data versus Monte Carlo differences in order to obtain an accurate prediction for the Far detector Monte Carlo.

Figures 9.9 and 9.10 show the nominal Monte Carlo in black and the superimposed Monte Carlo prediction in the Far detector for both CC-like and NC-like events. The three plots in each figure shows this comparison for Run I, Run II and combined total of both runs. One can compare the change in the Near detector 
Monte Carlo fit to the new Far detector predictions and see that they are changed in a analogous way to the Near.

\begin{tabular}{|c|c|c|c|c|}
\hline $\begin{array}{c}\text { Parameter } \\
\text { Type }\end{array}$ & $\begin{array}{c}\text { Parameter } \\
\text { Name }\end{array}$ & $\begin{array}{c}\text { Default } \\
\text { Value }\end{array}$ & $\begin{array}{c}\text { 1 Sigma } \\
\text { Value }\end{array}$ & $\begin{array}{c}\text { Near Detector } \\
\text { Fit Value }\end{array}$ \\
\hline \hline Neugen & CCMA & 1.0 & 0.15 & 0.976 \\
\hline Neugen & knor112122 & 1.0 & 0.33 & 1.097 \\
\hline Neugen & knor113123 & 1.0 & 0.2 & 1.247 \\
\hline Neugen & knor212222 & 1.0 & 0.33 & 0.846 \\
\hline Neugen & knor213223 & 1.0 & 0.2 & 1.025 \\
\hline Reconstruction & Shower Energy & 1.0 & 0.11 & 0.97 \\
\hline Reconstruction & Track Energy & 1.0 & 0.02 & 0.954 \\
\hline Reconstruction & Normalization & 1.0 & 0.04 & 1.004 \\
\hline Reconstruction & CC Background & 1.0 & 0.05 & 0.881 \\
\hline Reconstruction & NC Background & 1.0 & 0.50 & 1.391 \\
\hline
\end{tabular}

Table 9.1: Parameters results of fitting the Near Detector CC-like and NC-like energy spectra. 

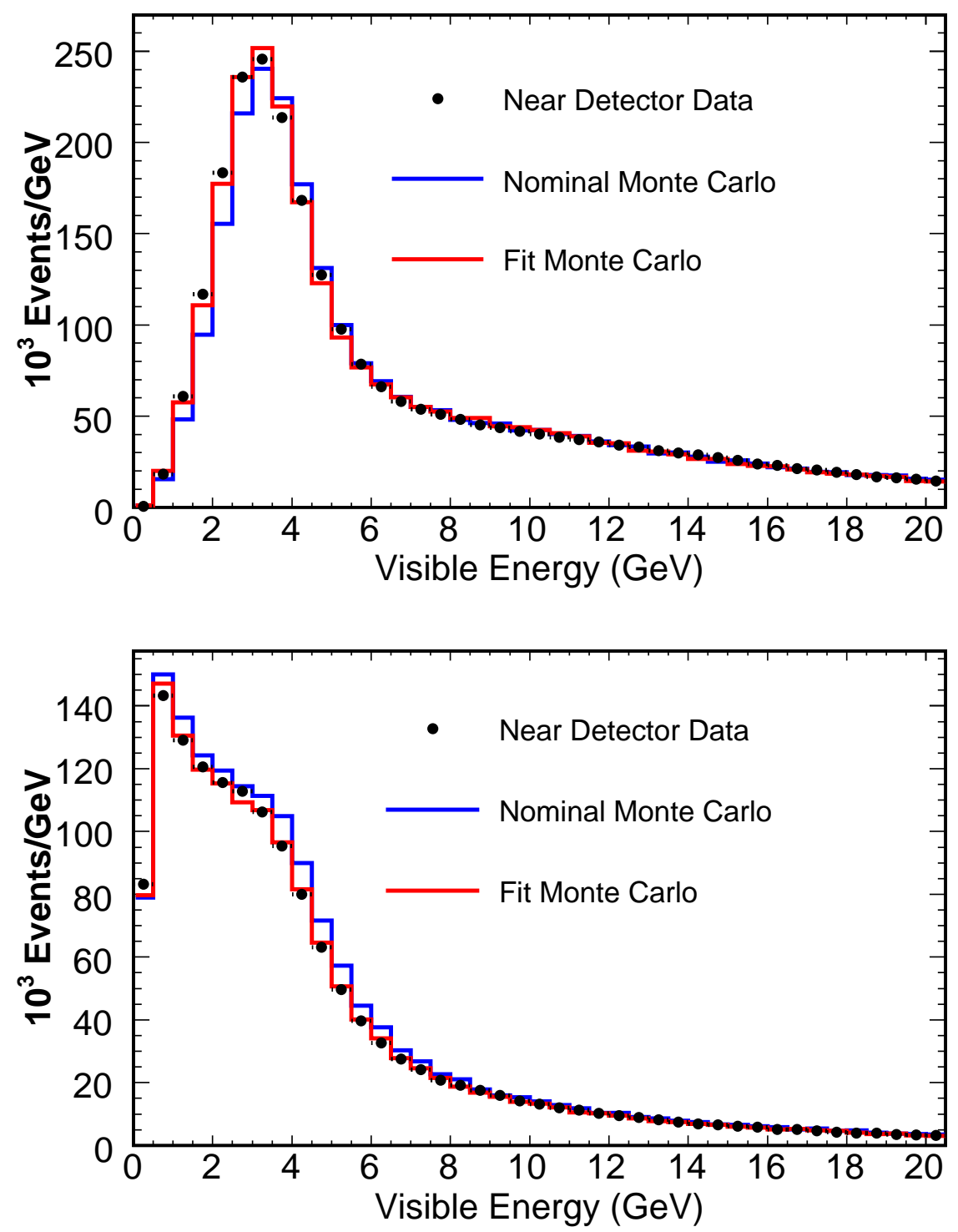

Figure 9.8: Results of fitting the Near detector charged current (top) and neutral current energy spectra (bottom). The black dots are the Near detector data, the blue spectra is the nominal Monte Carlo, and the red spectra is the best fit Monte Carlo to the data. 


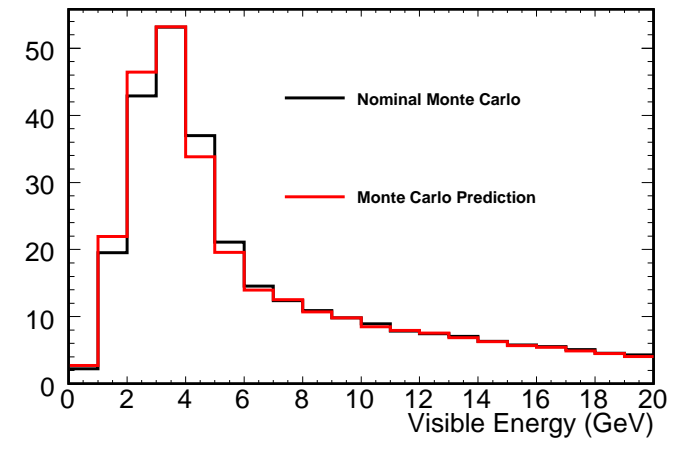

(a)

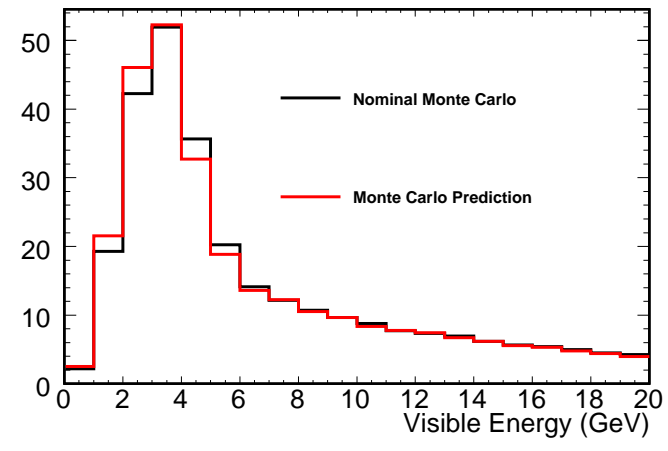

(b)

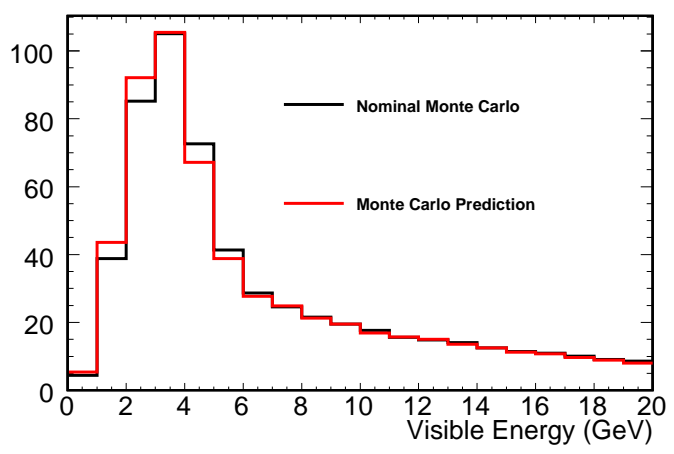

(c)

Figure 9.9: Far detector charged current nominal Monte Carlo in black, and the Monte Carlo prediction in red for (a) Run I (b) Run II (c) Run I combined with Run II. 


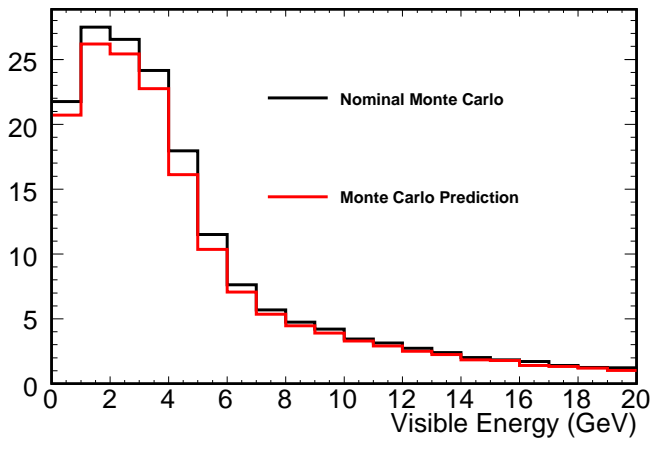

(a)

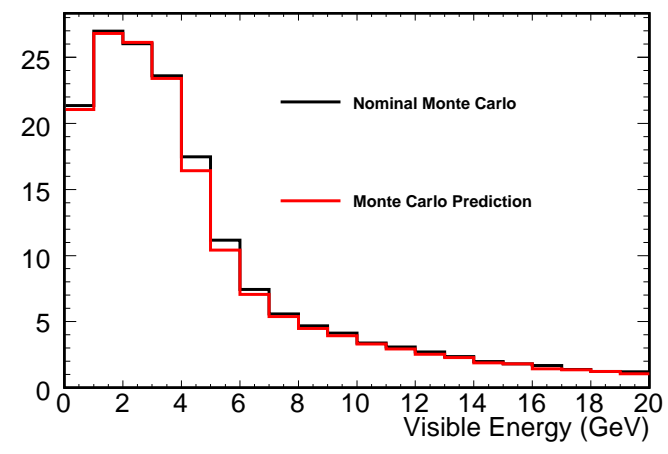

(b)

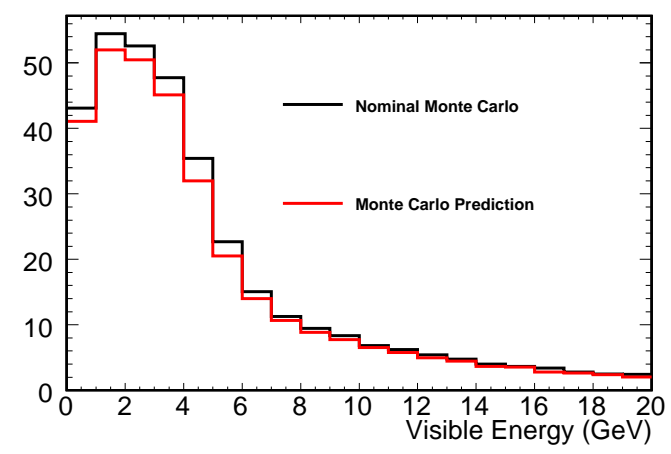

(c)

Figure 9.10: Far detector neutral current nominal Monte Carlo in black, and the Monte Carlo prediction in red for (a) Run I (b) Run II (c) Run I combined with Run II. 


\subsection{Measuring the sterile fraction}

Now that a prediction for the Far detector Monte Carlo in the absence of neutrino oscillations is complete, we can use this to perform a simultaneous fit to both the CC-like and NC-like energy spectra for the oscillation parameters.

This fit is essentially a grid search over acceptable ranges for the three oscillation parameters: $\Delta m^{2}, \sin ^{2}(2 \theta)$, and $f_{\text {sterile }}$. There are no systematic nuisance parameters incorporated into the fit, whose only affect will be to increase the size our of resultant parameter space contours. It is not expected to contribute significantly to the fit since our statistical errors are much larger than the systematic.

Figure 9.11 shows the Run I, Run II, and combined energy spectra for CClike events in the Far detector. The black dots are the data, the blue spectrum is the Monte Carlo prediction and the red spectra is the best fit spectrum under a oscillation hypothesis. Superimposed are the NC background in green, the best fit to the NC background in dashed green, and the tau appearance component in purple.

Immediately after this in Figure 9.12 is the $68 \%, 90 \%$, and $99 \%$ confidence level contours for $\Delta m^{2}$ versus $\sin ^{2}(2 \theta)$ for Run I, Run II, and combined running. The best fit parameters for Run I were $\Delta m^{2}=2.665 \times 10^{-3} \mathrm{eV}^{2} / \mathrm{c}^{4}$ and $\sin ^{2}(2 \theta)=1.0$. The best fit parameters for Run II were $\Delta m^{2}=2.395 \times 10^{-3}$ $\mathrm{eV}^{2} / \mathrm{c}^{4}$ and $\sin ^{2}(2 \theta)=1.0$. The final best fit parameters for the combined data set is $\Delta m^{2}=2.525 \times 10^{-3} \mathrm{eV}^{2} / \mathrm{c}^{4}$ and $\sin ^{2}(2 \theta)=1.0$, which is completely consistent with the most recent charged current analysis results of $\Delta m^{2}=2.38 \times 10^{-3}$ $\mathrm{eV}^{2} / \mathrm{c}^{4}$. The differences are entirely statistical due to there being slight differences in the number of events selected. 
Figure 9.13 shows the Run I, Run II, and combined energy spectra for NClike events in the Far detector. The black dots are the data, the blue spectrum is the Monte Carlo prediction and the red spectra is the best fit spectrum under a oscillation hypothesis. Superimposed are the CC background in green, the best fit to the $\mathrm{CC}$ background in dashed green, and the tau appearance component in purple. Immediately after this in Figure 9.14 is the $68 \%, 90 \%$, and $99 \%$ confidence level contours for $\Delta m^{2}$ versus $f_{\text {sterile }}$ for Run I, Run II, and combined running. The best fit parameters for Run I were $\Delta m^{2}=2.665 \times 10^{-3} \mathrm{eV}^{2} / \mathrm{c}^{4}$ and $f_{\text {sterile }}=0.415$. The best fit parameters for Run II were $\Delta m^{2}=2.395 \times 10^{-3} \mathrm{eV}^{2} / \mathrm{c}^{4}$ and $f_{\text {sterile }}=0.335$. The final best fit parameters for the combined data set is $\Delta m^{2}=2.525 \times 10^{-3}$ $\mathrm{eV}^{2} / \mathrm{c}^{4}$ and $f_{\text {sterile }}=0.375$. Note the large extent of the $f_{\text {sterile }}$ contour. It is apparent the sterile analysis is very statistics limited and a non-zero measurement for $f_{\text {sterile }}$ is not to be taken as a positive signal since $f_{\text {sterile }}=0.0$ is entirely consistent with our contours. The results of 100 individual Mock Data Challenge results with an input value of $f_{\text {sterile }}=0.0$ from Chapter 8 shows that the best fit has a significant statistical fluctuation with one subrun giving a best fit of 0.9.

The number of events for each component of the energy spectra in Figures 9.11 and 9.13 are tabulated in Appendix C for both Neutral Current and Charged Current events for Run I, Run II, and combined running. 


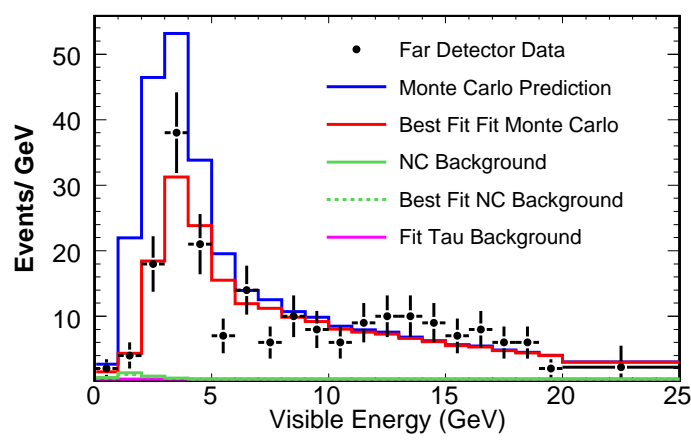

(a)

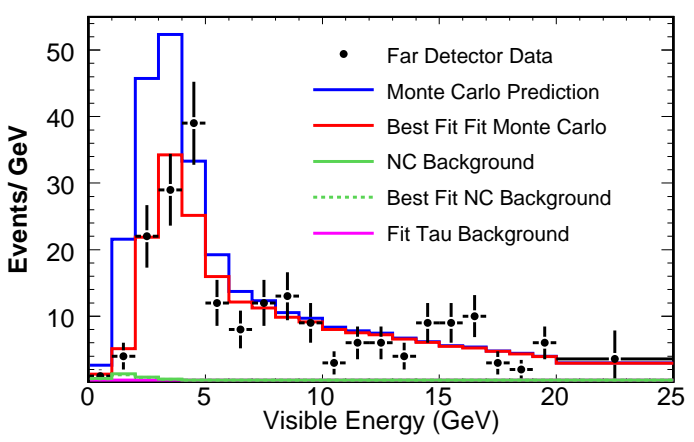

(b)

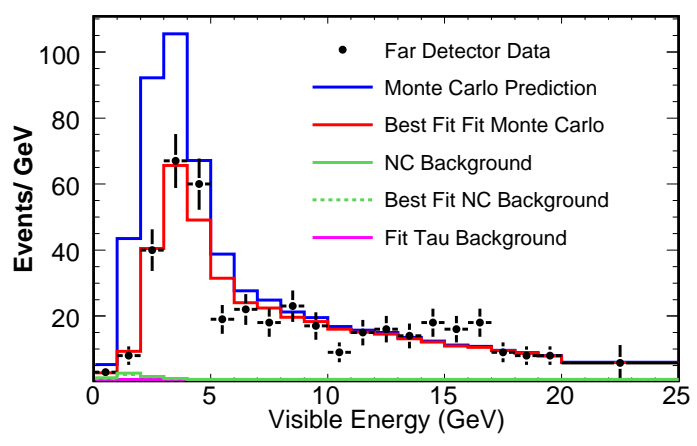

(c)

Figure 9.11: The black dots are the Far detector charged current data, the blue spectrum is the far detector predicted Monte Carlo and the red spectrum is the best fit under a oscillation hypothesis. The solid green spectrum is the neutral current background and the dashed green is the fit background. The purple spectrum is the fit tau appearance component. Each plot corresponds to (a) Run I (b) Run II (c) Run I combined with Run II. 


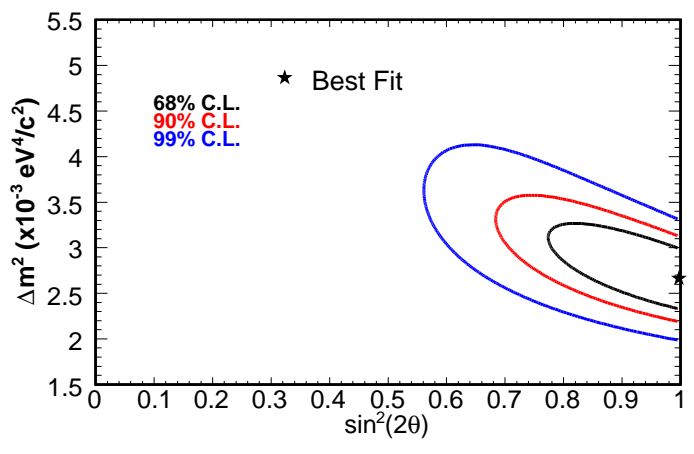

(a)

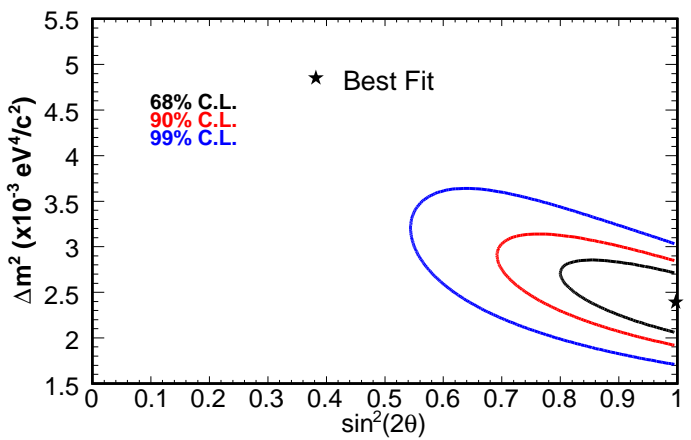

(b)

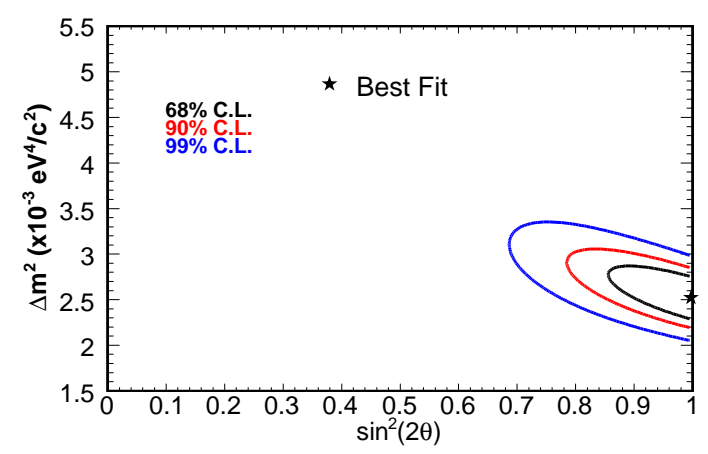

(c)

Figure 9.12: The 68\%, 90\%, and 99\% confidence level contours for a oscillation measurement of $\Delta m^{2}$ versus $\sin ^{2}(2 \theta)$. Each plots corresponds to (a) Run I (b) Run II (c) Run I combined with Run II. 


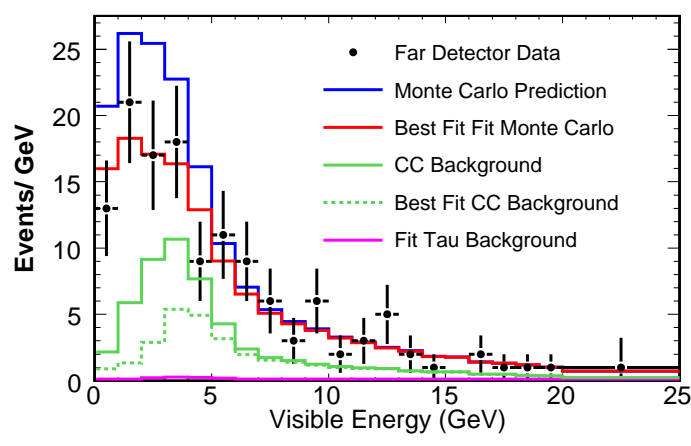

(a)

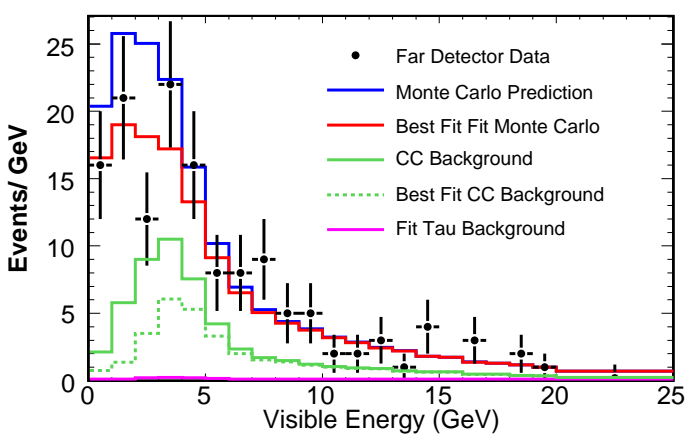

(b)

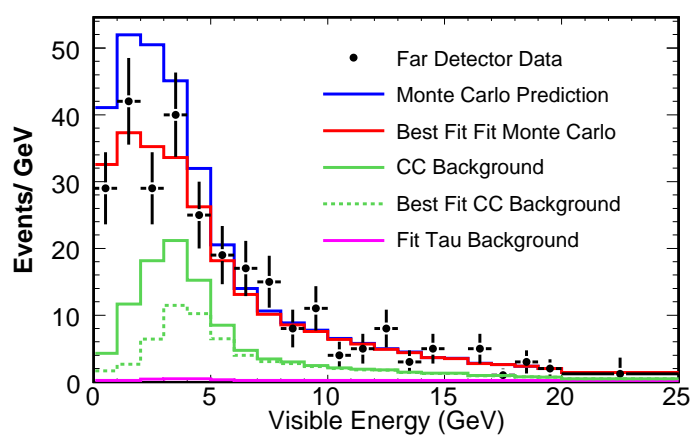

(c)

Figure 9.13: The black dots are the Far detector neutral current data, the blue spectrum is the far detector predicted Monte Carlo and the red spectrum is the best fit under a oscillation hypothesis. The solid green spectrum is the charged current background and the dashed green is the fit background. The purple spectrum is the fit tau appearance component. Each plot corresponds to (a) Run I (b) Run II (c) Run I combined with Run II. 


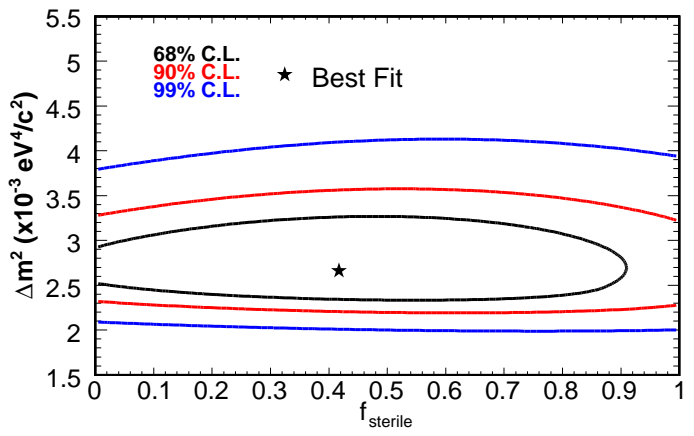

(a)

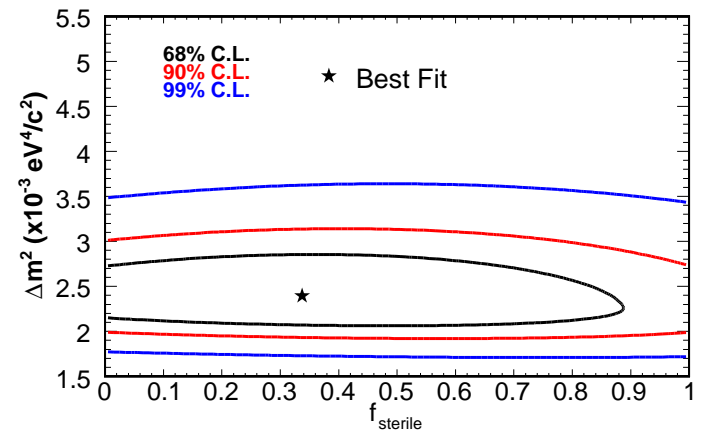

(b)

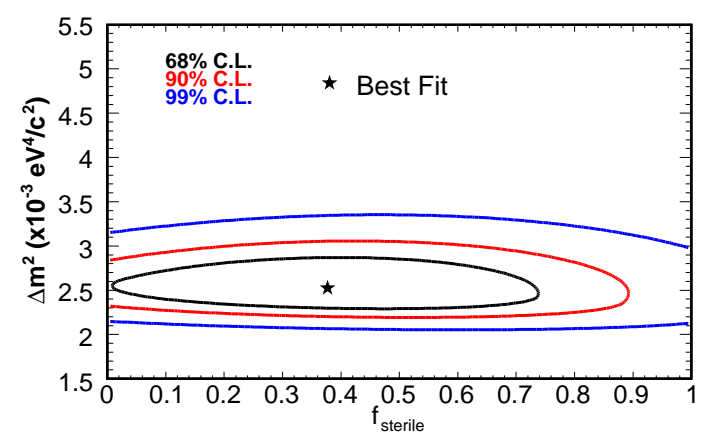

(c)

Figure 9.14: The 68\%, 90\%, and 99\% confidence level contours for a oscillation measurement of $\Delta m^{2}$ versus $f_{\text {sterile }}$. Each plots corresponds to (a) Run I (b) Run II (c) Run I combined with Run II. 


\subsection{Conclusions}

The final one dimensional contour for $f_{\text {sterile }}$ is shown in Figure 9.15. Superimposed are the $69 \%, 90 \%, 99 \%$ confidence level contours. Notice the large extent of the contours themselves. With the level of statistical error, it is not possible to rule out any values at the $99 \%$ confidence level. At $90 \%$ confidence level, we can say that $f_{\text {sterile }}<0.77$, implying that pure sterile oscillations can be ruled out at $90 \%$ confidence level. Further statistics are needed to improve this limit.

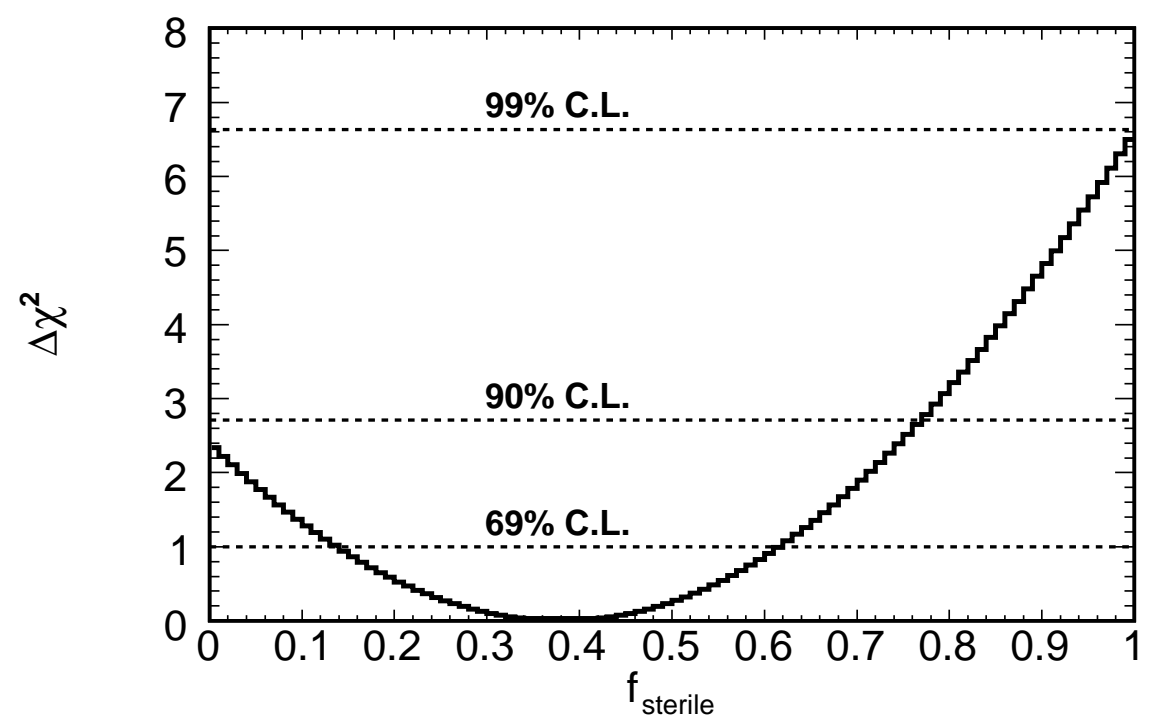

Figure 9.15: One dimensional $\Delta \chi^{2}$ contour for the $f_{\text {sterile }}$ measurement. 


\section{Appendix A}

\section{Example of Slicing Algorithm}

In this appendix, we show the results of slicing the example beam spill used in chapter 4 in Figure 4.2. This particular beam spill produced 16 separate slices, which are documented in Table A.1. The first column refers to the slice number, while the second column is the true neutrino energy given in $\mathrm{GeV}$. The third column tells whether the event was a charged current or neutral current interaction and the last two columns give the completeness and purity of each slice as defined in Chapter 4.

We plot the transverse position versus longitudinal position of all the strips in each slice in Figures A.1 through A.18. This includes strips from both views, so we are in effect superimposing two 2D figures on top of one another. The color of the strips corresponds to the total pulse height in ADC counts, given by the color scale to the right of each plot.

If one examines the individual slices, we find interesting features that can be understood by looking at the completeness and purity. We only discuss slices that deviate significantly from 1 in both completeness and purity.

If one looks at Figures A.2, A.4, A.7, A.13, A.14, A.15, and A.18 we find slices that all have a very high completeness, but purities that deviate from one. 
Table A.1: Results of slicing Figure 4.2

\begin{tabular}{|c|c|c|c|c|}
\hline Slice Number & True Energy $(\mathrm{GeV})$ & CC/NC & Completeness & Purity \\
\hline \hline Slice 0 & 3.94 & CC & 1 & 0.99 \\
\hline Slice 1 & 17.01 & CC & 0.96 & 0.63 \\
\hline Slice 2 & 3.86 & CC & 1 & 0.98 \\
\hline Slice 3 & 1.76 & CC & 1 & 0.86 \\
\hline Slice 4 & 6.27 & CC & 1 & 0.99 \\
\hline Slice 5 & 3.40 & NC & 1 & 0.99 \\
\hline Slice 6 & 2.64 & CC & 1 & 0.78 \\
\hline Slice 7 & 1.25 & CC & 1 & 0.97 \\
\hline Slice 8 & 3.72 & CC & 0.92 & 0.98 \\
\hline Slice 9 & 3.72 & CC & 0.08 & 0.92 \\
\hline Slice 10 & 16.32 & CC & 1 & 0.86 \\
\hline Slice 11 & 3.56 & CC & 1 & 0.74 \\
\hline Slice 12 & 6.87 & NC & 0.99 & 0.49 \\
\hline Slice 13 & 2.13 & CC & 1 & 0.97 \\
\hline Slice 14 & 5.07 & NC & 1 & 0.99 \\
\hline Slice 15 & 59.30 & CC & 0.99 & 0.76 \\
\hline
\end{tabular}

In each of these cases, one can see two individual interactions found in the slice. By eye, one notices that they are generally spatially separated and downstream reconstructed software can pick further separate these events by looking at more detailed information in tracks and showers.

Of interest is Figures A.9 and A.10. These two slices came from the same charged current neutrino interaction. This is why slice 9 has a low completeness of 0.08 . The other $92 \%$ of the neutrino's energy is in slice 8 . The reason for this is because the muon that was produced and properly put into slice 8 was captured in either the steel or scintillator and decayed approximately two microseconds later, producing a electron which deposited a fraction of the neutrinos total energy in the detector. We illustrate this point more by looking at the timing distribution of the two slices in Figure A.11. The black spike corresponds to the initial neutrino 
interaction, while the red comes from the electron produced from muon decay. In addition, Figure A.12 plots the longitudinal position versus time and one clearly sees that the second slice occurs exactly at the longitudinal position where muon track in the first slice ends, indicating evidence for muon decay.

\section{A.1 Conclusions}

The following event displays show that the Slicing algorithm can properly isolate individual interactions in the Near detector with minimal bias. The results of the intensity studies in Chapter 6 further give credence to the robustness of the algorithm. 


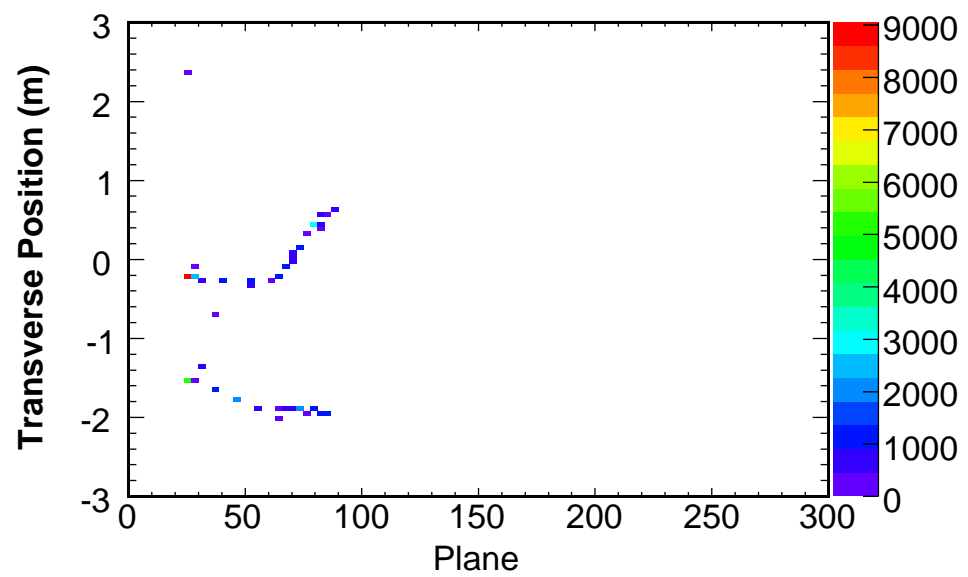

Figure A.1: Slice 0 is a charged current event with an energy of $3.94 \mathrm{GeV}$ reconstructed with a completeness of 1 and a purity of 0.99 .

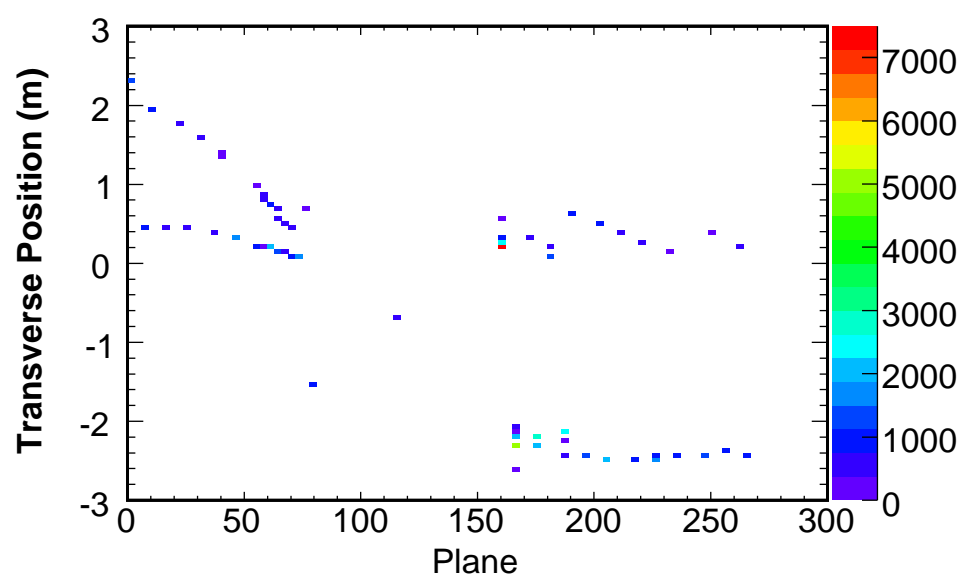

Figure A.2: Slice 1 is a charged current event with an energy of $17.01 \mathrm{GeV}$ reconstructed with a completeness of 0.96 and a purity of 0.63 . This event only has a track that penetrates the front face of the detector is identified as a rock muon from a event whose true vertex is outside the detector. 


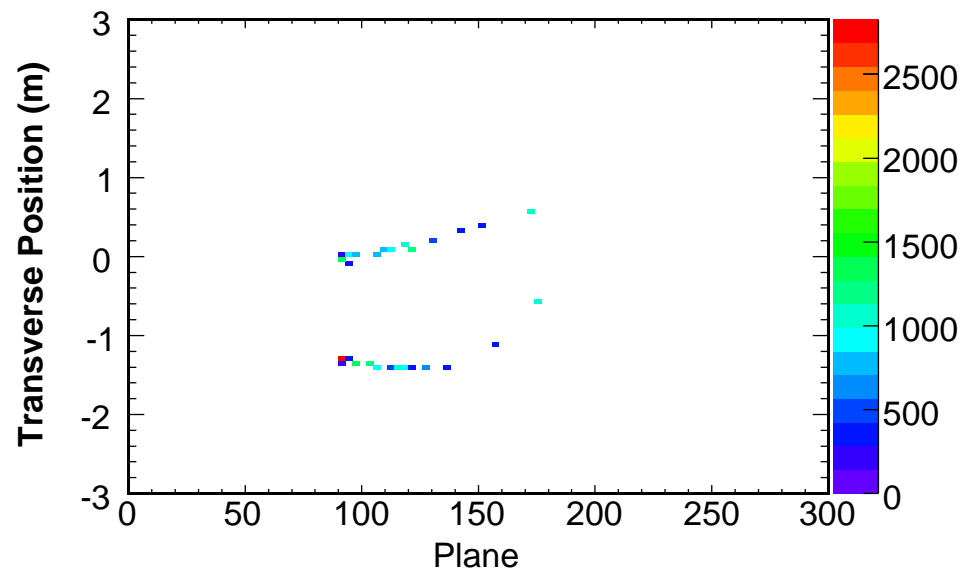

Figure A.3: Slice 2 is a charged current event with an energy of $3.86 \mathrm{GeV}$ reconstructed with a completeness of 1 and a purity of 0.98 .

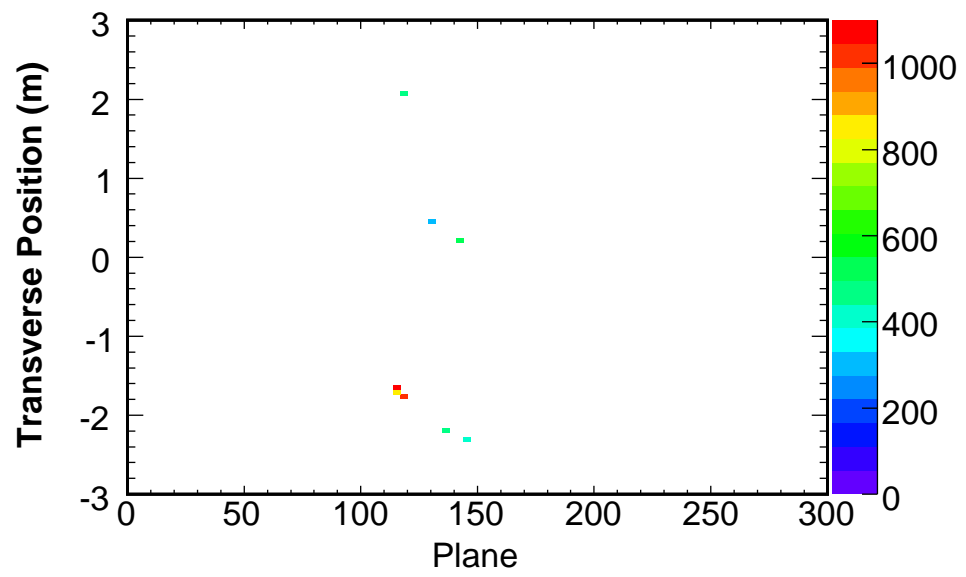

Figure A.4: Slice 3 is a charged current event with an energy of $1.76 \mathrm{GeV}$ reconstructed with a completeness of 1 and a purity of 0.86 . 


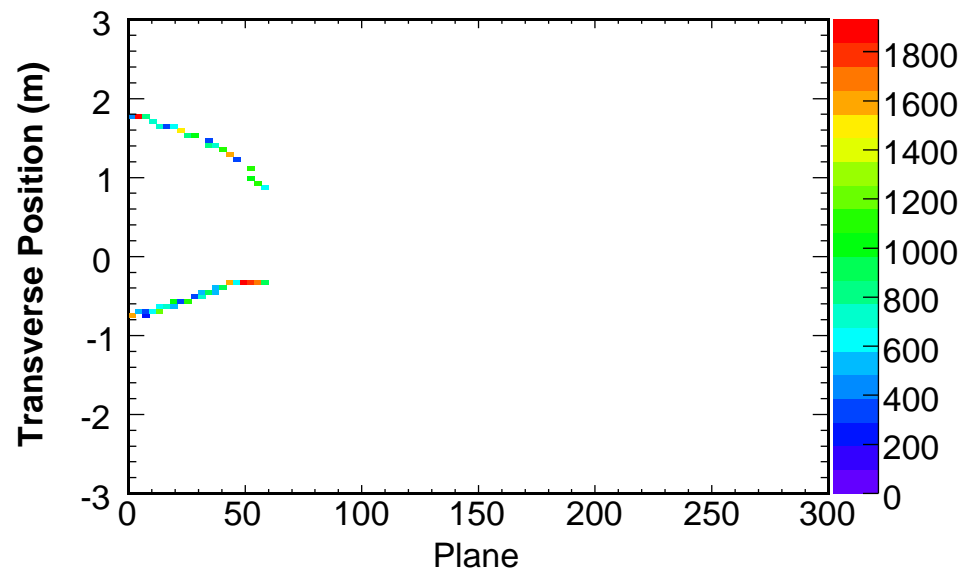

Figure A.5: Slice 4 is a charged current event with an energy of $6.27 \mathrm{GeV}$ reconstructed with a completeness of 1 and a purity of 0.99 .

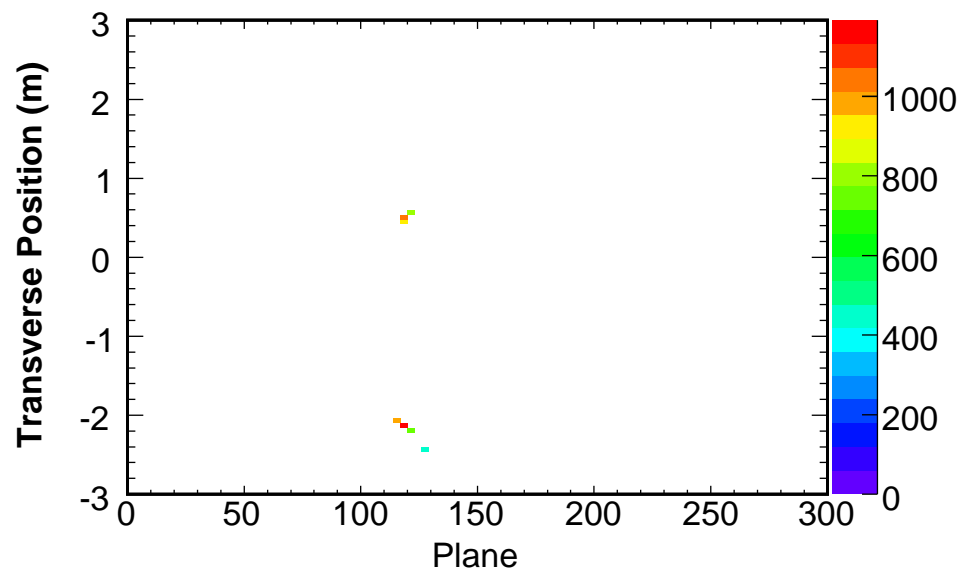

Figure A.6: Slice 5 is a neutral current event with an energy of $3.40 \mathrm{GeV}$ reconstructed with a completeness of 1 and a purity of 0.99 . 


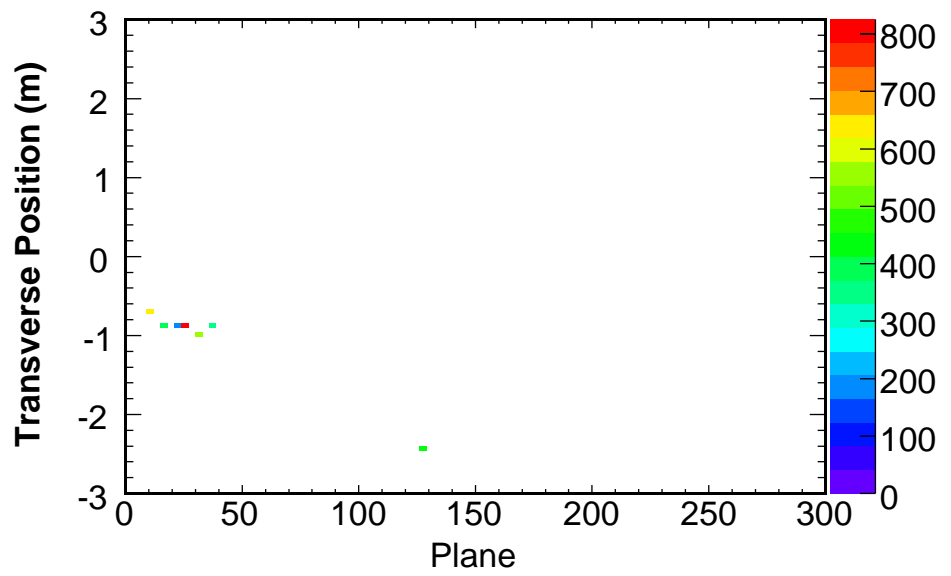

Figure A.7: Slice 6 is a charged current event with an energy of $2.64 \mathrm{GeV}$ reconstructed with a completeness of 1 and a purity of 0.78 .

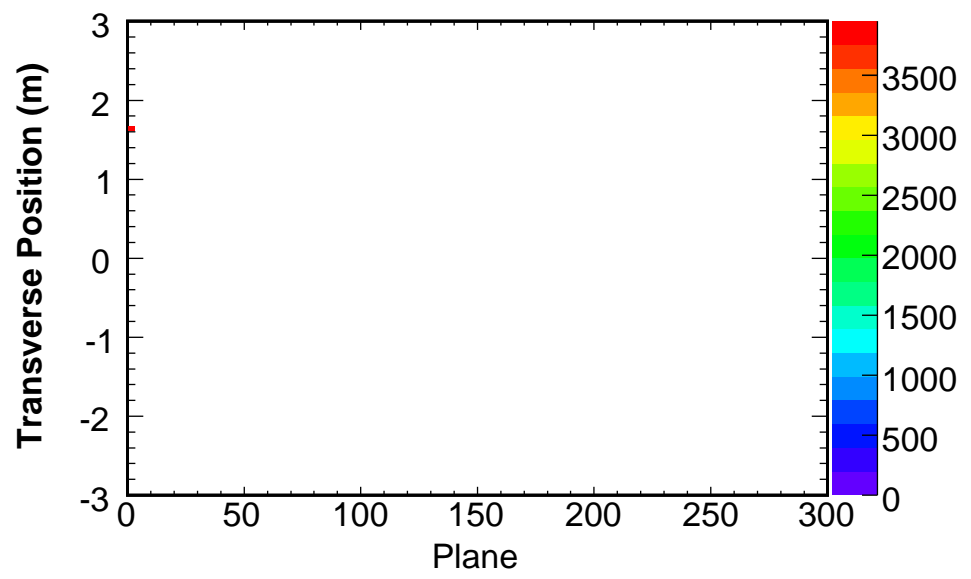

Figure A.8: Slice 7 is a charged current event with an energy of $1.25 \mathrm{GeV}$ reconstructed with a completeness of 1 and a purity of 0.97 . 


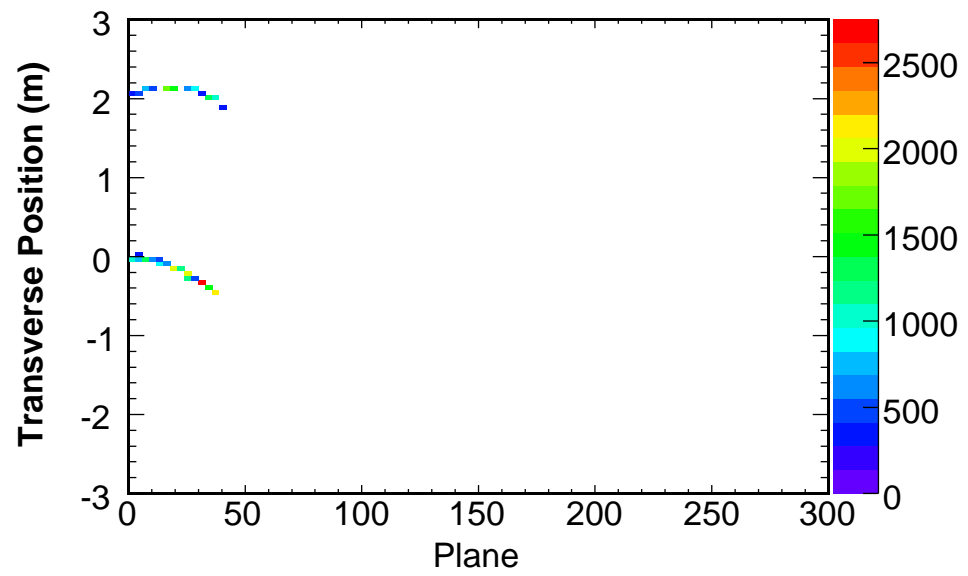

Figure A.9: Slice 8 is a charged current event with an energy of $3.72 \mathrm{GeV}$ reconstructed with a completeness of 0.92 and a purity of 0.98 .

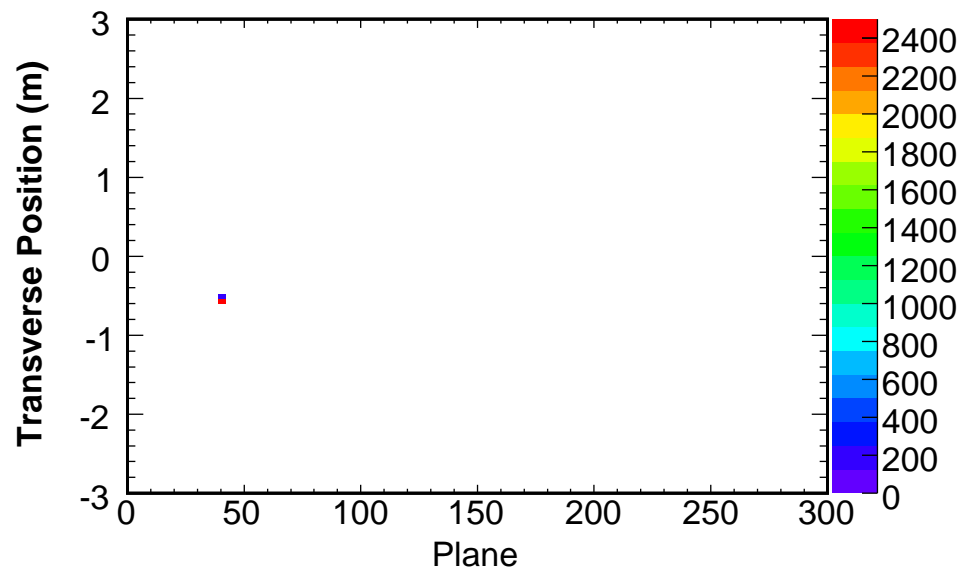

Figure A.10: Slice 9 is a charged current event with an energy of $3.72 \mathrm{GeV}$ reconstructed with a completeness of 0.08 and a purity of 0.92 . 


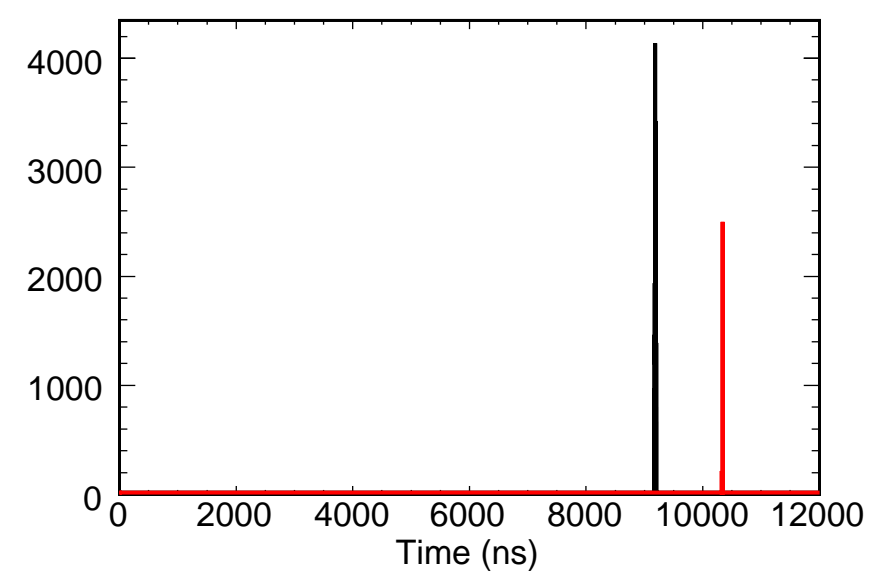

Figure A.11: The timing distribution of slices 8 (black) and 9 (red). The red spike corresponds to a electron from the decay of the captured muon.

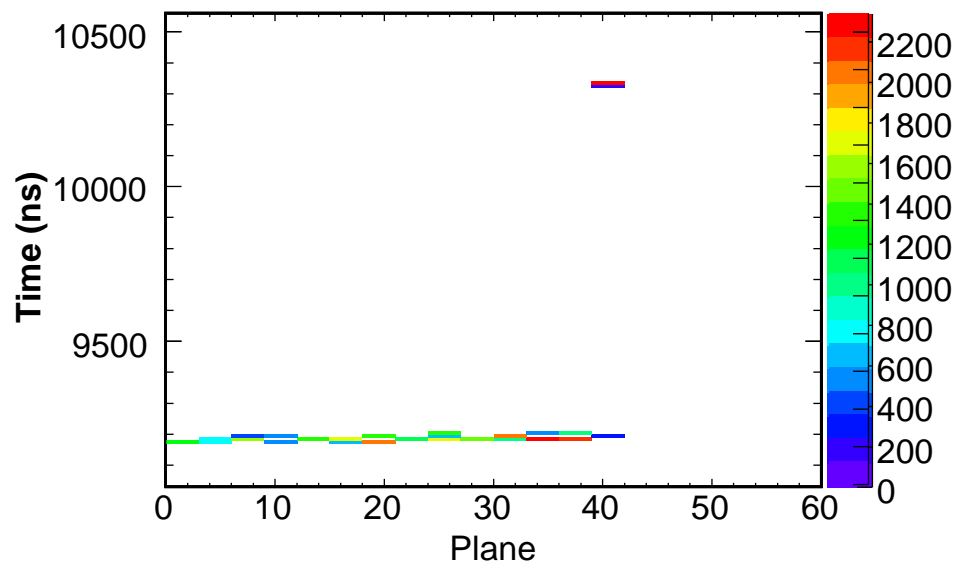

Figure A.12: The longitudinal position versus time of slices 8 and 9 . 


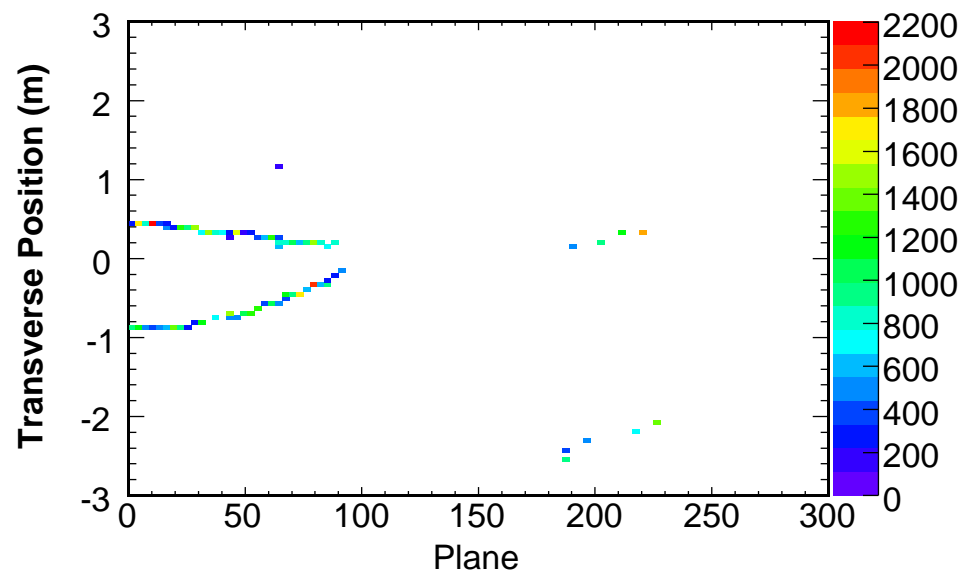

Figure A.13: Slice 10 is a charged current event with an energy of $16.32 \mathrm{GeV}$ reconstructed with a completeness of 1 and a purity of 0.86 .

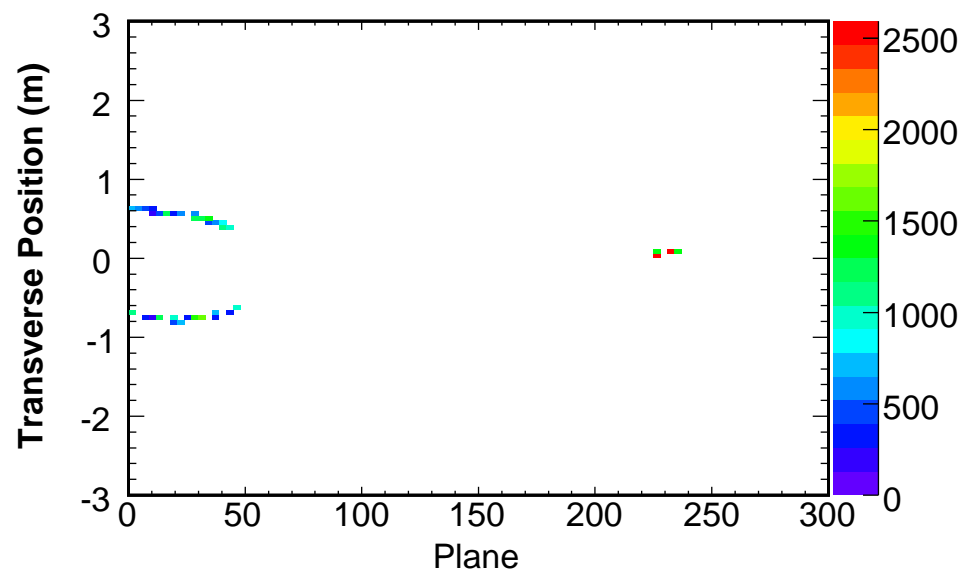

Figure A.14: Slice 11 is a charged current event with an energy of $3.56 \mathrm{GeV}$ reconstructed with a completeness of 1 and a purity of 0.74 . 


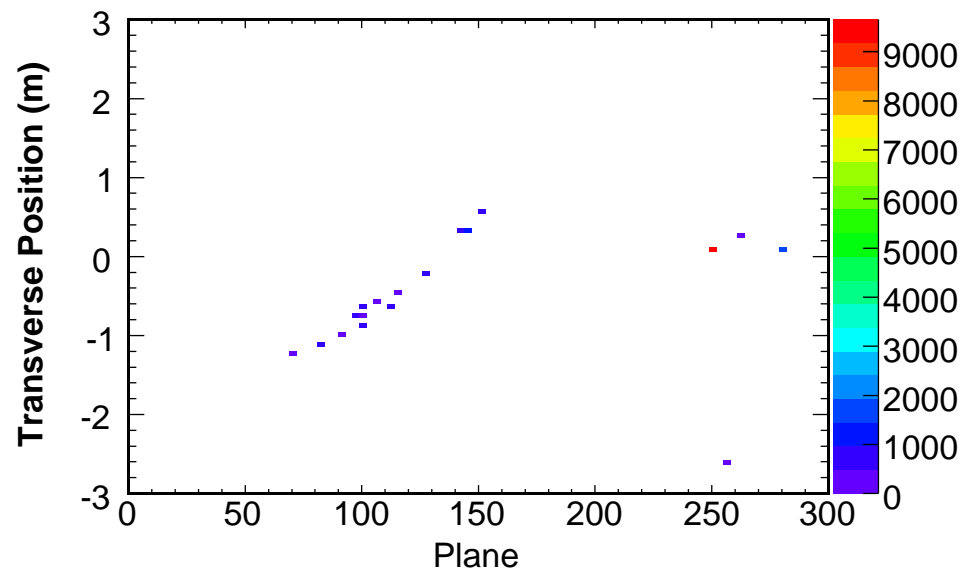

Figure A.15: Slice 12 is a neutral current event with an energy of $6.87 \mathrm{GeV}$ reconstructed with a completeness of 0.99 and a purity of 0.49 .

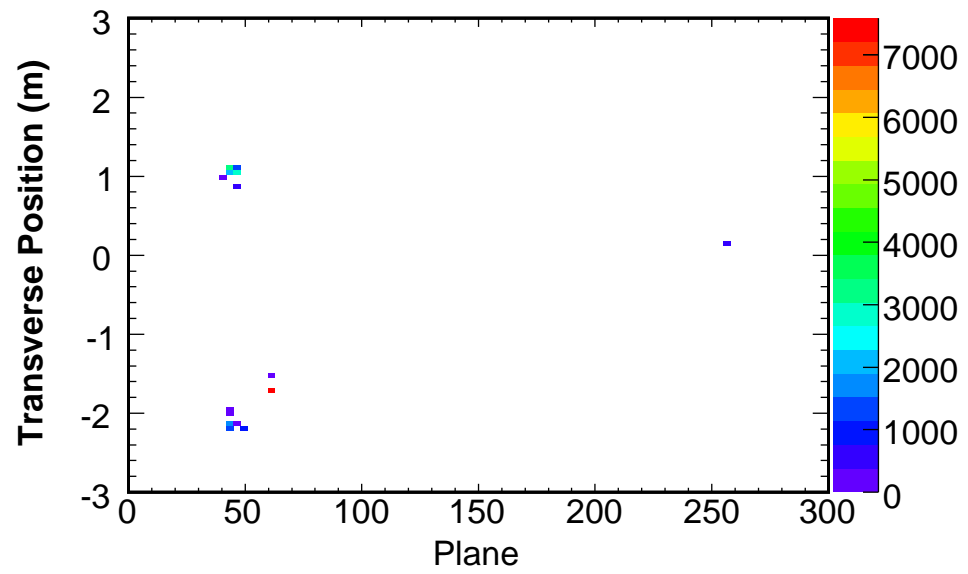

Figure A.16: Slice 13 is a charged current event with an energy of $2.13 \mathrm{GeV}$ reconstructed with a completeness of 1 and a purity of 0.97 . 


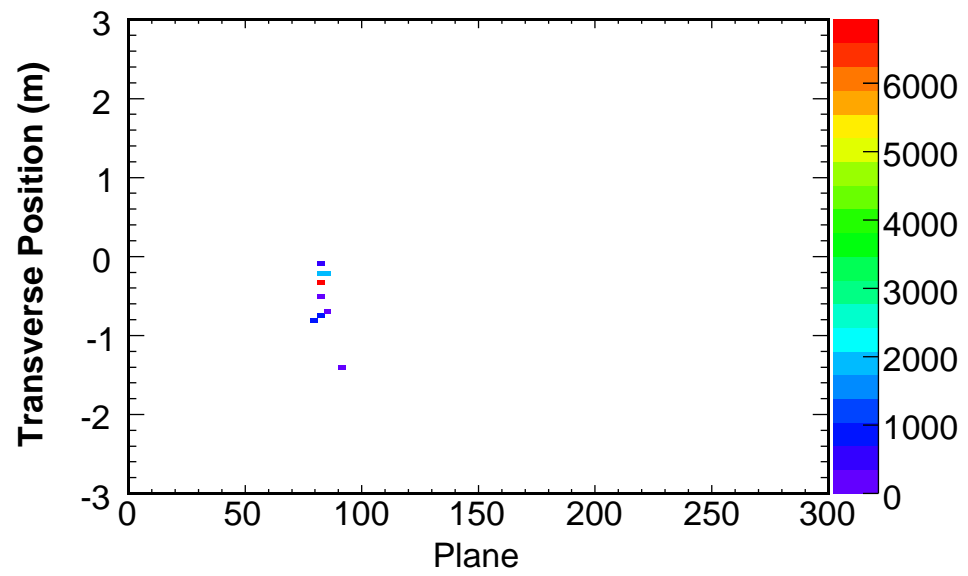

Figure A.17: Slice 14 is a neutral current event with an energy of $5.07 \mathrm{GeV}$ reconstructed with a completeness of 1 and a purity of 0.99 .

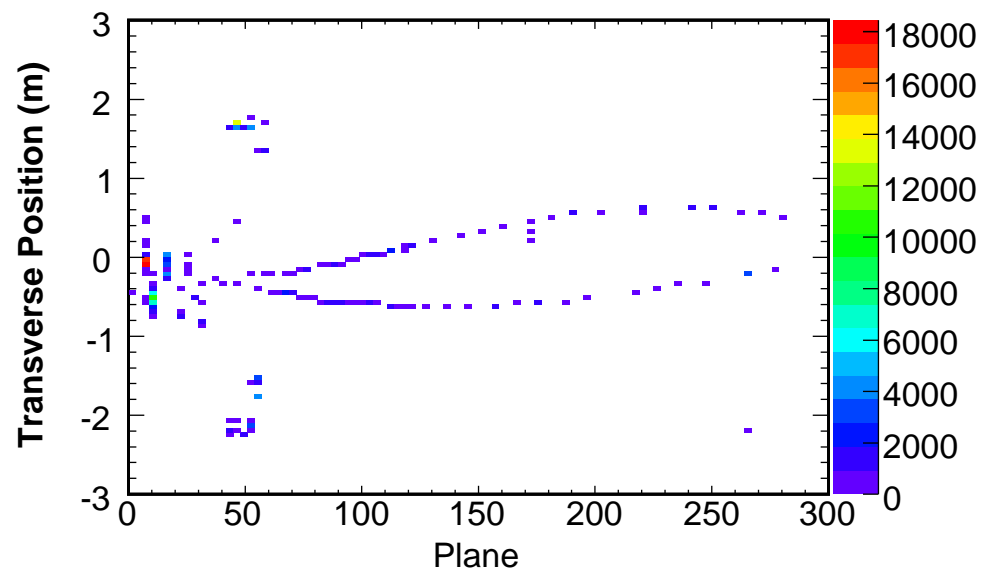

Figure A.18: Slice 15 is a charged current event with an energy of $59.30 \mathrm{GeV}$ reconstructed with a completeness of 0.99 and a purity of 0.76 . 


\section{Appendix B}

\section{Systematic Parameters used in NDFit}

We document in this appendix the effects of 10 systematic parameters on the neutral current and charged current energy spectra in the Near detector from Monte Carlo. Each of these 10 parameters will be used in the NDFit to resolve differences between data and Monte Carlo. Each plot has the charged current energy spectra in the top left and the neutral current energy spectra in the bottom left. Superimposed are the energy spectra produced by changing the parameter by $\pm 1,2,3 \sigma$. The right two plots show the ratio of the nominal Monte Carlo to the changed spectrum. 

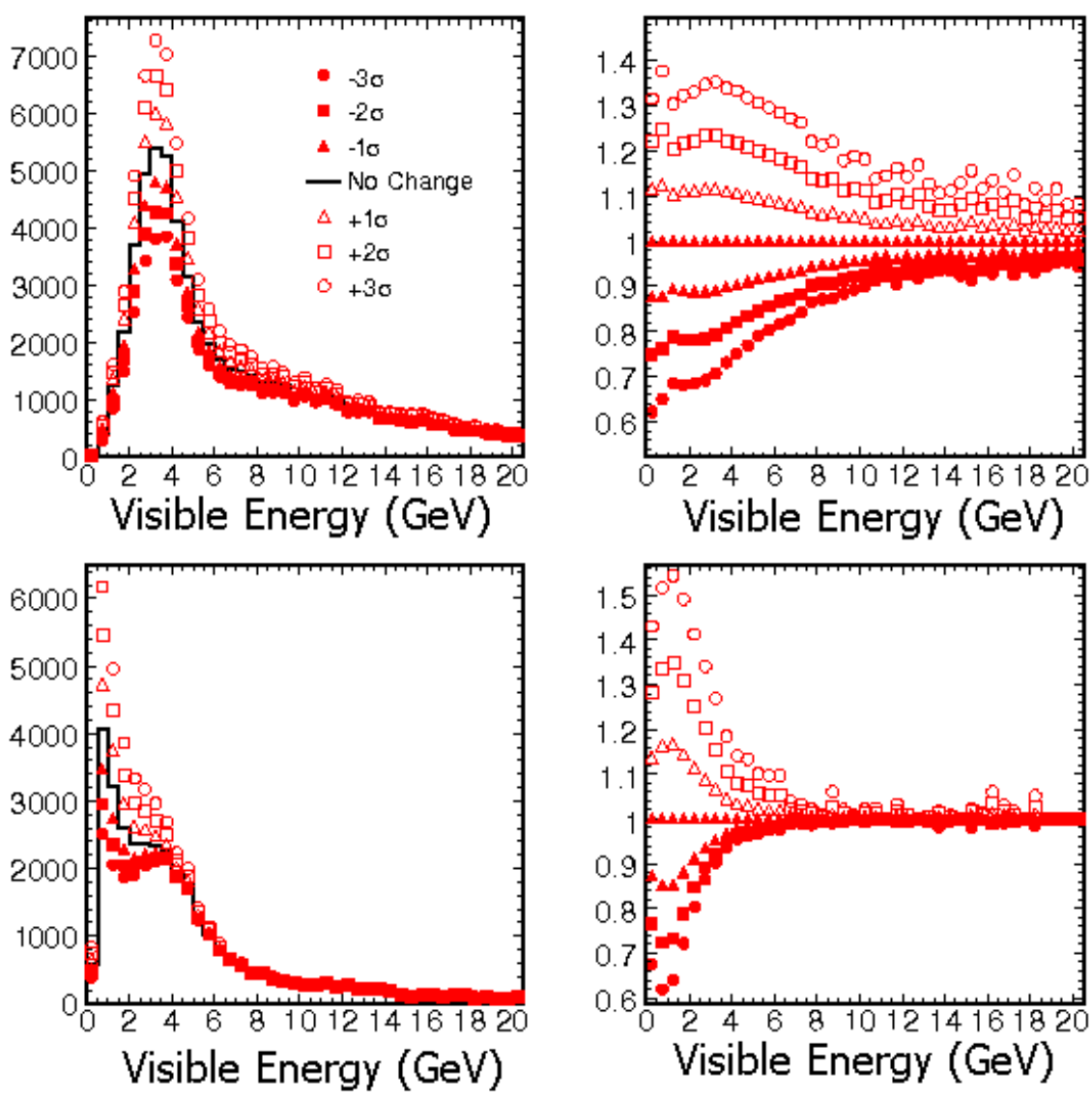

Figure B.1: The effect of changing CCMA by $\pm 1,2,3 \sigma$ errors. 

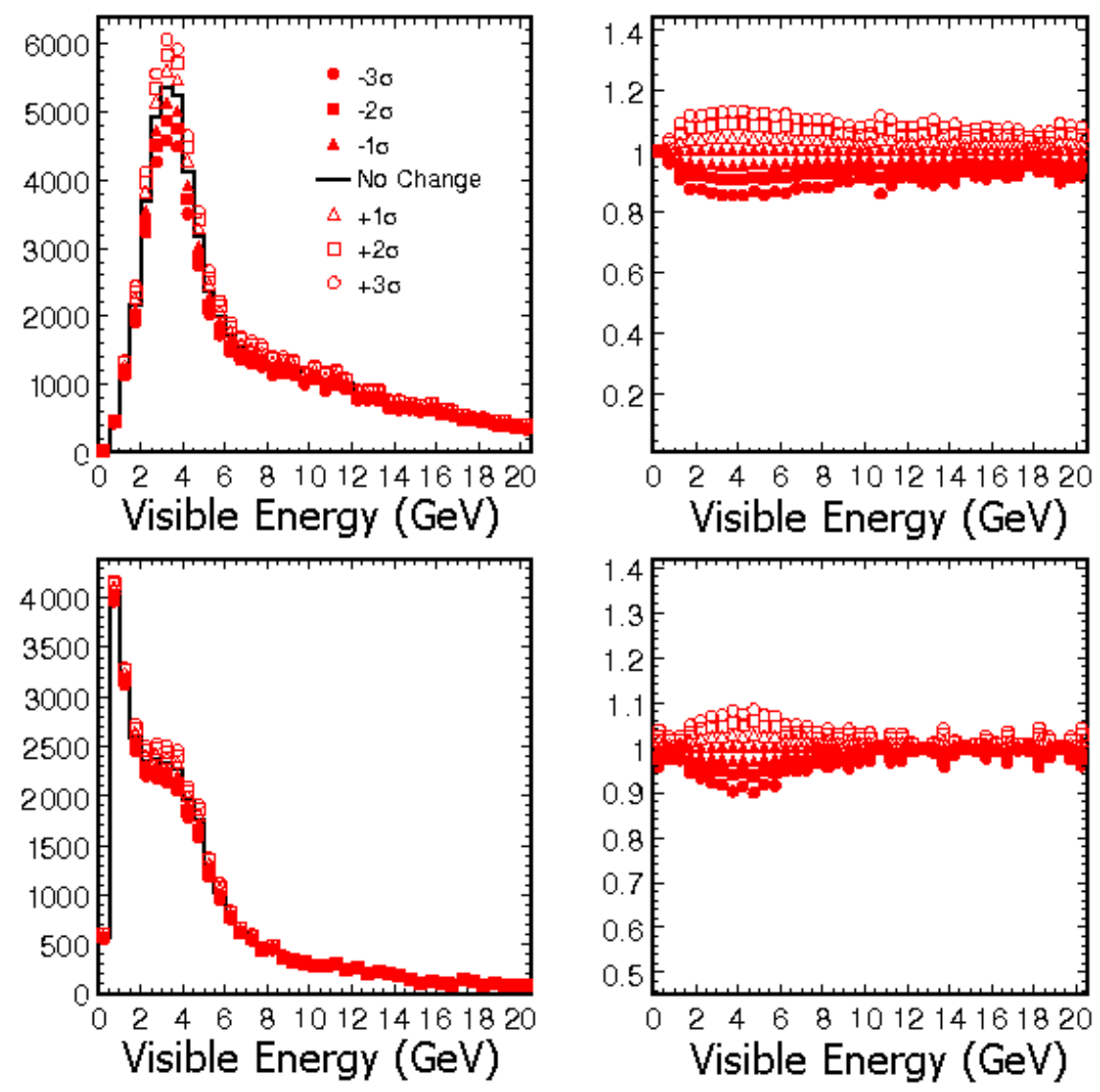

Figure B.2: The effect of changing $\mathrm{kno}_{112122}$ by $\pm 1,2,3 \sigma$ errors. 

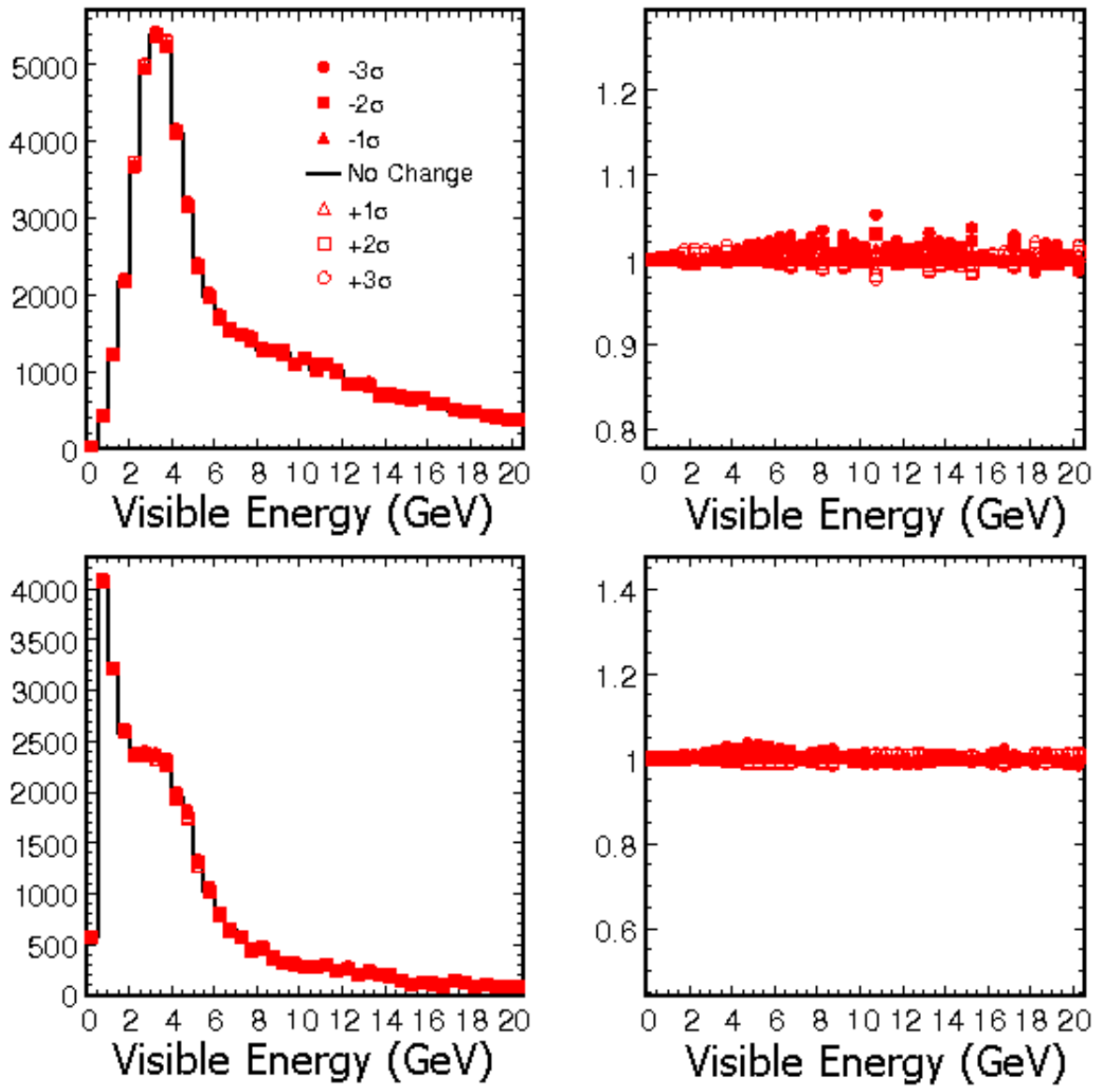

Figure B.3: The effect of changing $\mathrm{kno}_{113123}$ by $\pm 1,2,3 \sigma$. 

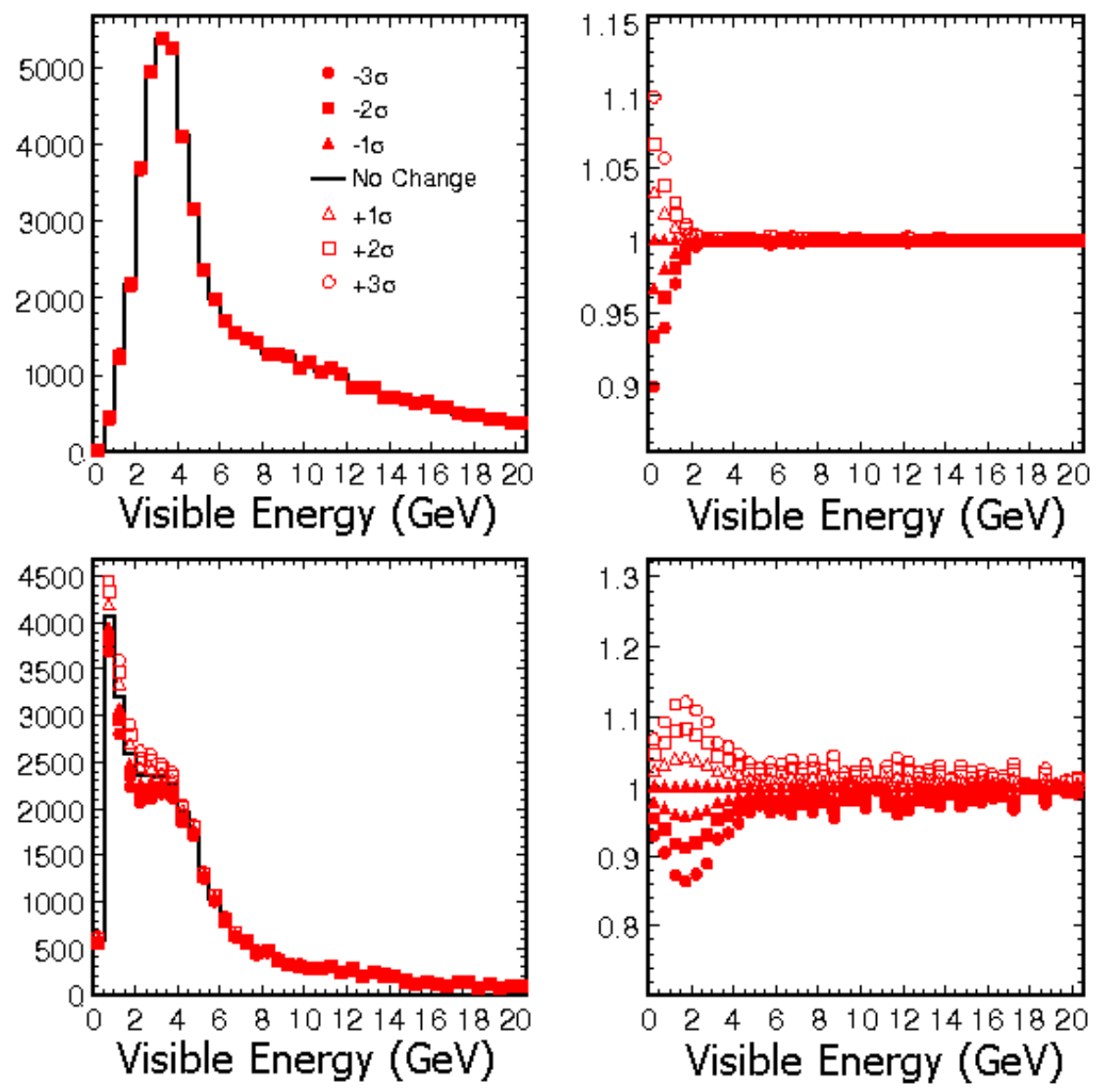

Figure B.4: The effect of changing $\mathrm{kno}_{212222}$ by $\pm 1,2,3 \sigma$. 

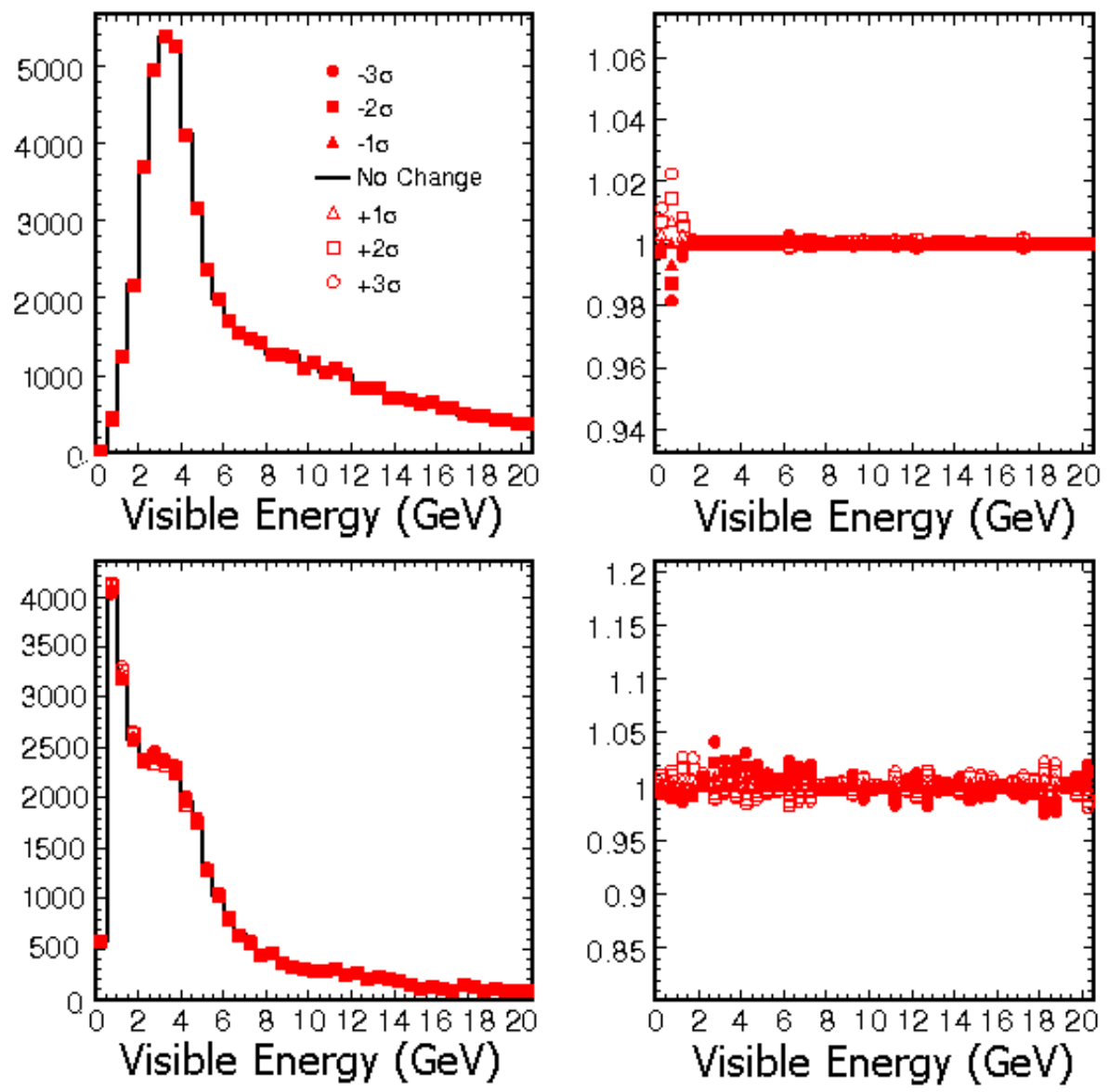

Figure B.5: The effect of changing $\mathrm{kno}_{r 213223}$ by $\pm 1,2,3 \sigma$. 

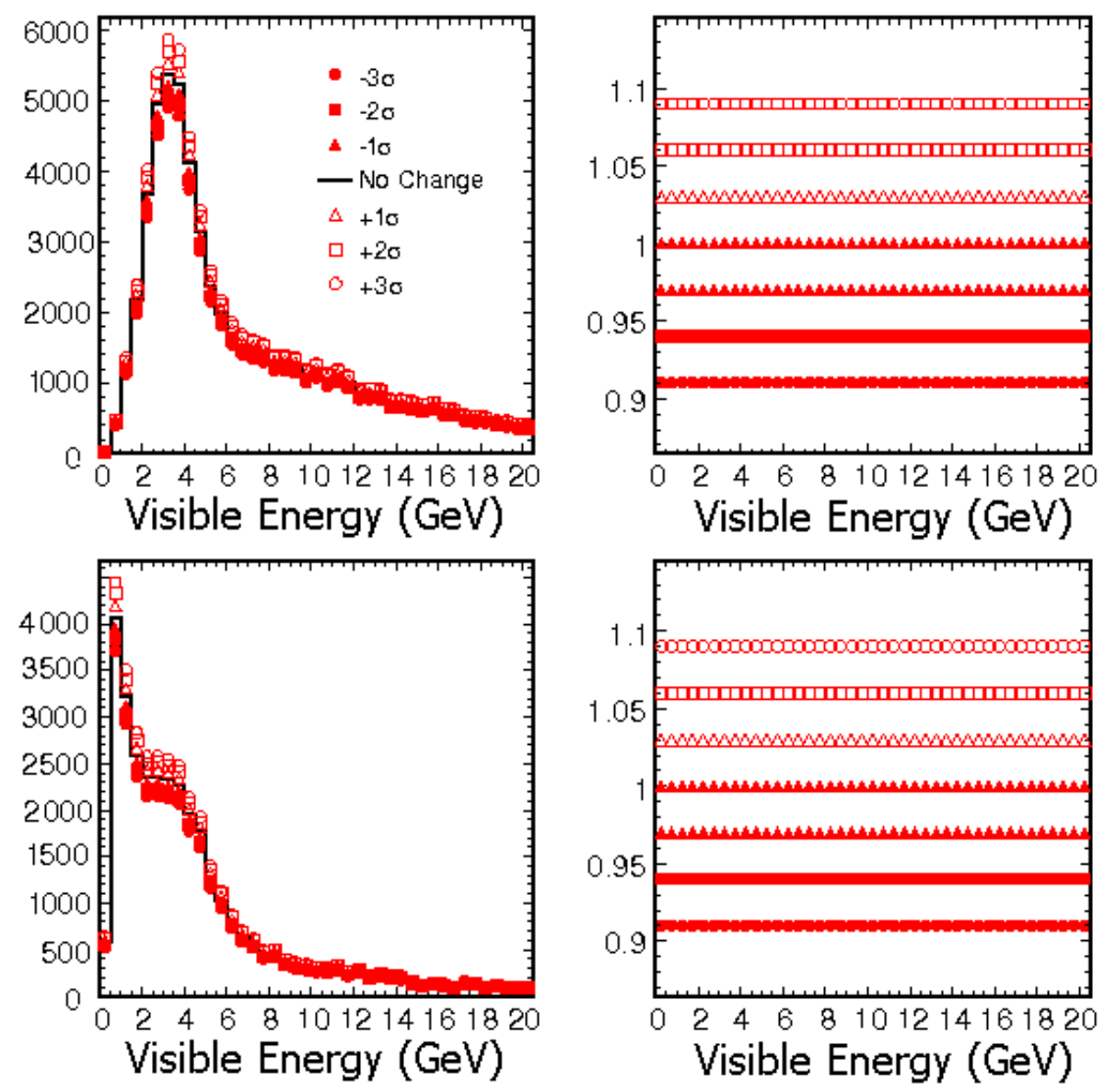

Figure B.6: The effect of changing the overall normalization by $\pm 1,2,3 \sigma$. 

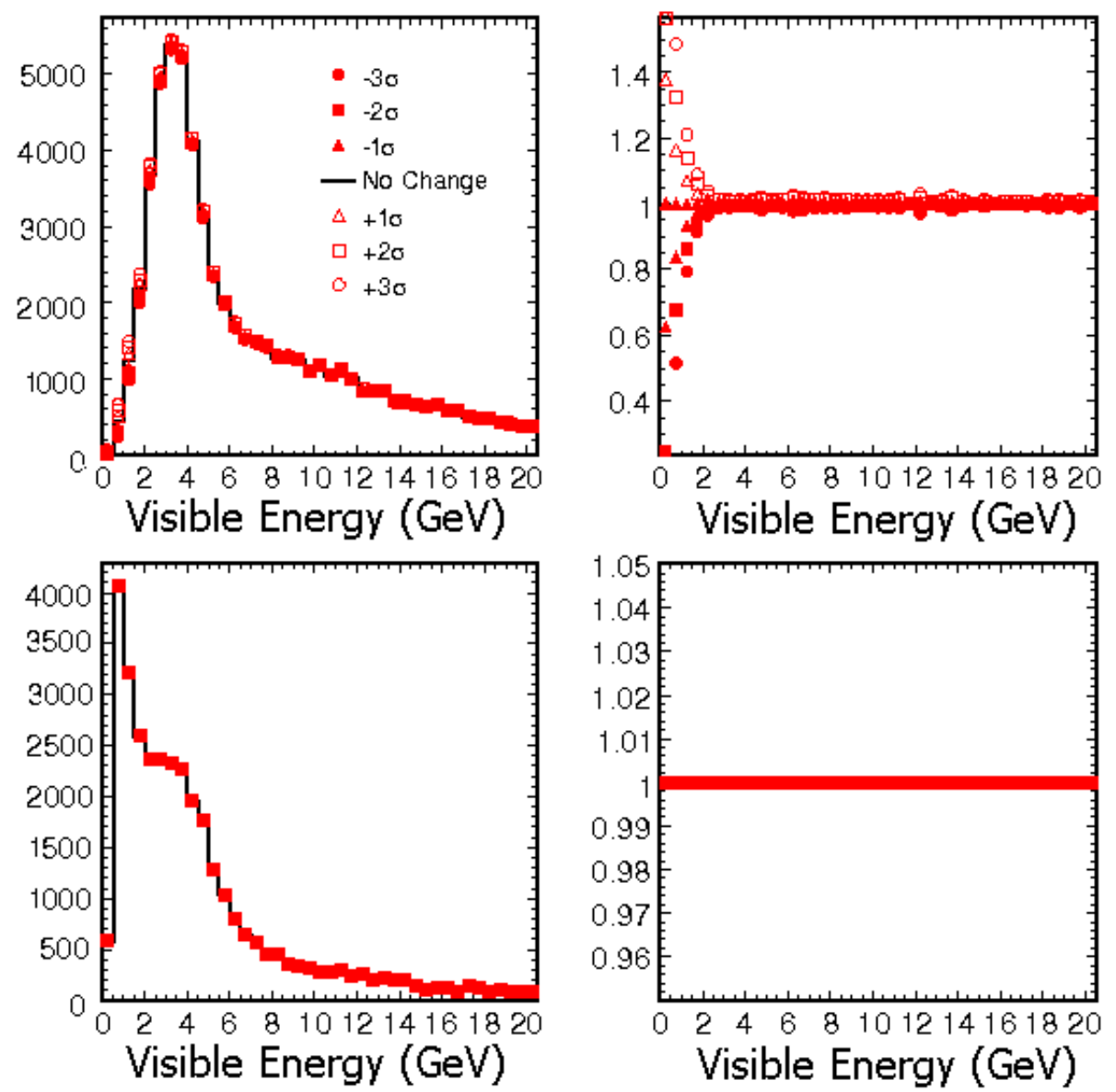

Figure B.7: The effect of changing the neutral current background in the charged current energy spectrum by $\pm 1,2,3 \sigma$. 

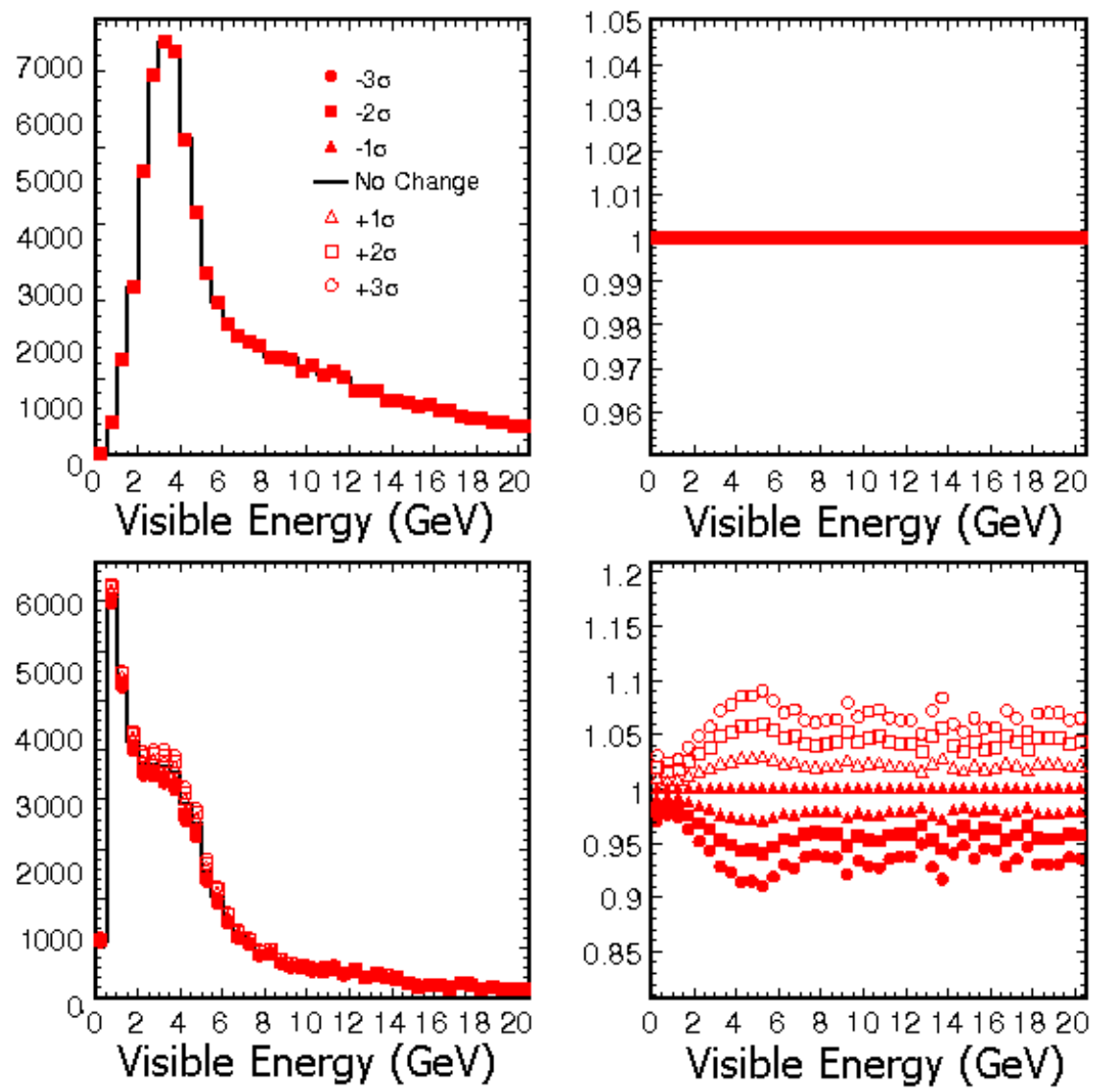

Visible Energy (GeV)

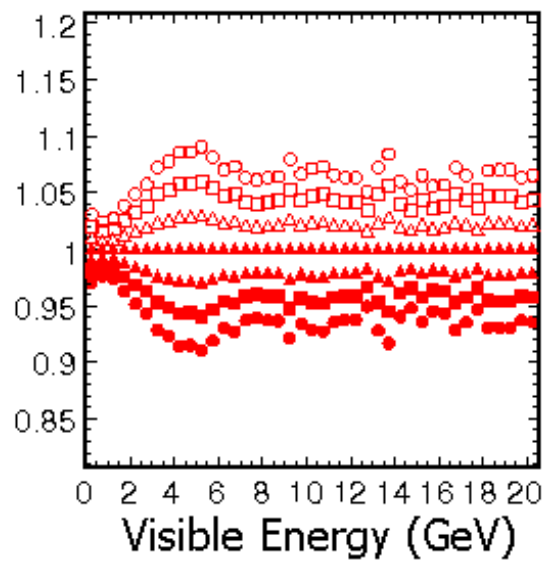

Figure B.8: The effect of changing the charged current background in the neutral current energy spectra by $\pm 1,2,3 \sigma$. 

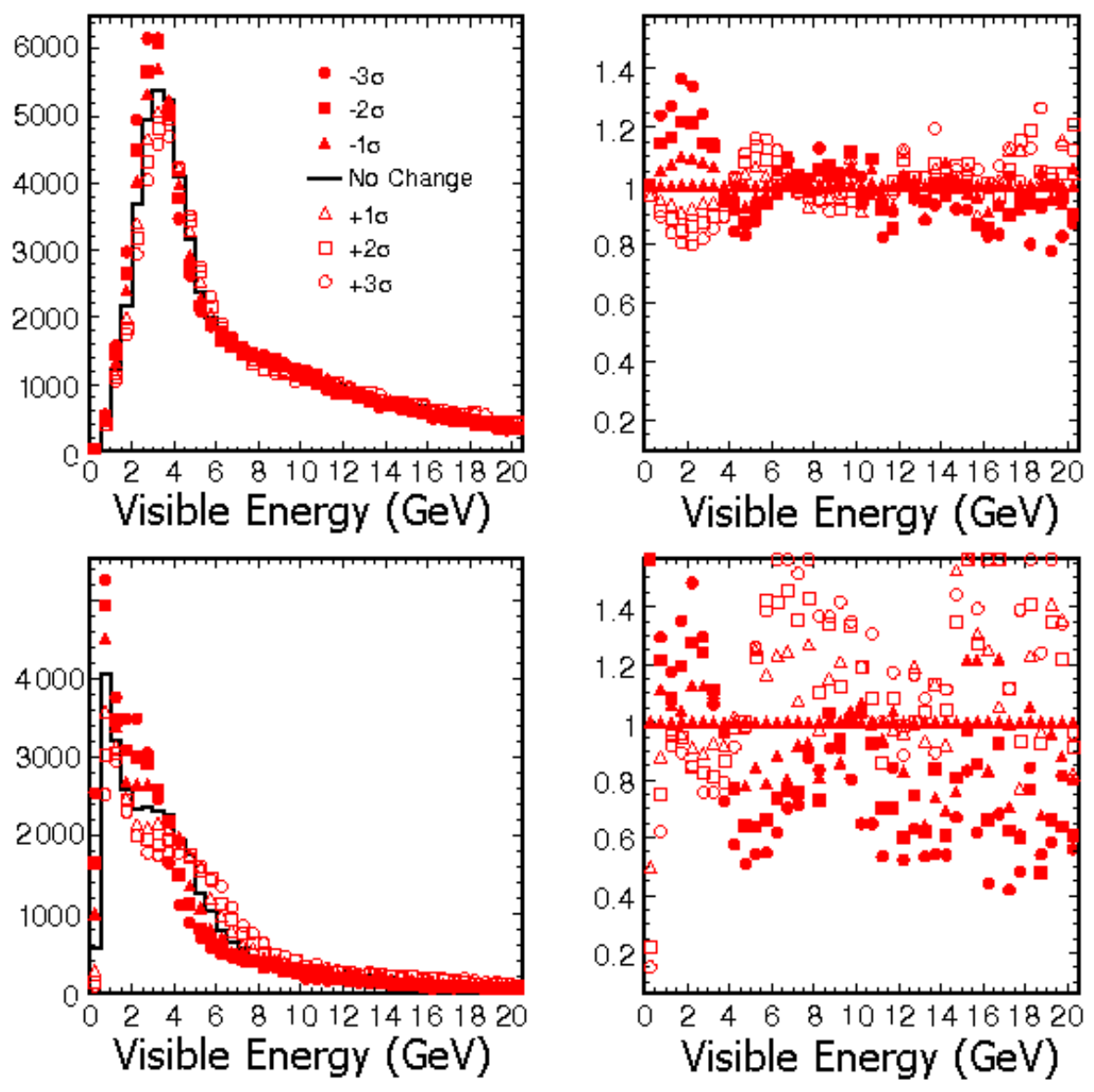

Figure B.9: The effect of changing the shower energy by $\pm 1,2,3 \sigma$. 

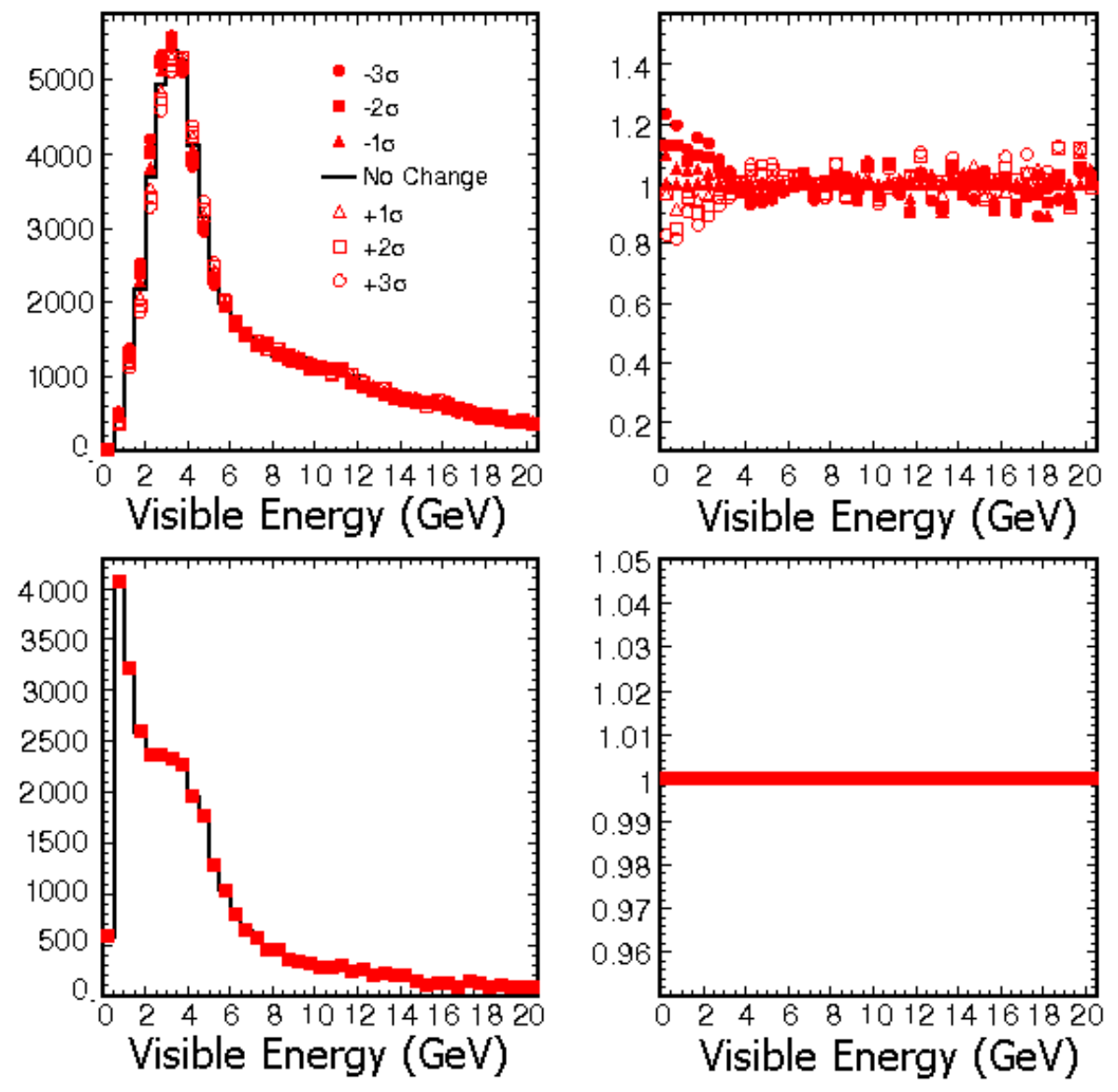

Figure B.10: The effect of changing the track energy by $\pm 1,2,3 \sigma$. 


\section{Appendix C}

\section{Far Detector Energy Spectra}

\section{Composition}

In six tables this appendix outlines the number of events in the final data and Monte Carlo samples for Charged Current and Neutral Current energy spectra in the Far detector for Run I, Run II and combined running. The information is shown in Tables 1 and 2 for Run I, Tables 3 and 4 for Run II, and Tables 5 and 6 for combined running. The first column labeled Energy Bin is the central value of the bin. If the central value is 0.5 , the bin covers $0<\mathrm{E}<1 \mathrm{GeV}$. 


\begin{tabular}{|c|c|c|c|c|c|c|c|c|c|c|c|c|c|c|c|c|c|c|c|c|c|}
\hline 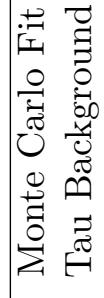 & 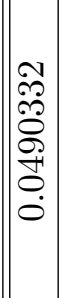 & 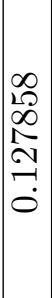 & $\begin{array}{l}\infty \\
2 \\
2 \\
\mathscr{2} \\
\stackrel{2}{+} \\
0 \\
0\end{array}$ & 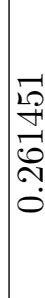 & 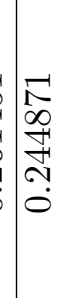 & 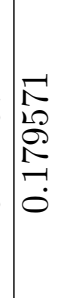 & 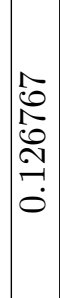 & 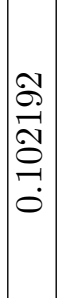 & 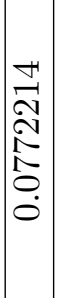 & \begin{tabular}{l}
$\infty$ \\
0 \\
0 \\
0 \\
\hdashline \\
0 \\
0 \\
0 \\
0
\end{tabular} & 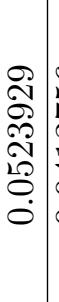 & 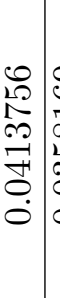 & 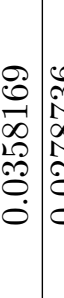 & 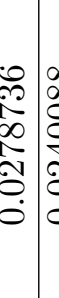 & 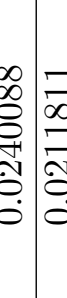 & 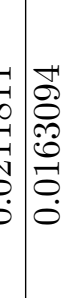 & 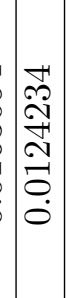 & $\begin{array}{c}\vec{H} \\
\stackrel{-}{\Xi} \\
\vec{\Xi} \\
\overrightarrow{0} \\
\dot{0}\end{array}$ & 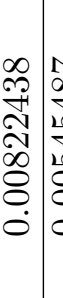 & & 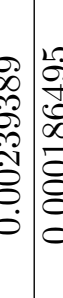 \\
\hline 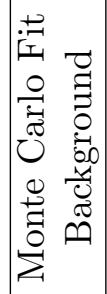 & $\mid \begin{array}{l}\overrightarrow{1} \\
\dot{0} \\
0 \\
2 \\
0 \\
0 \\
0 \\
0 \\
\end{array}$ & 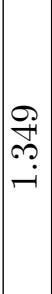 & $\begin{array}{l}\overrightarrow{\tilde{O}} \\
\dot{8} \\
\dot{\delta} \\
\dot{0} \\
\dot{v}\end{array}$ & 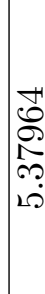 & 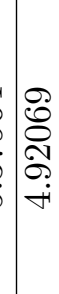 & 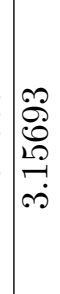 & 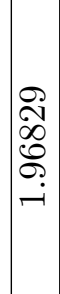 & 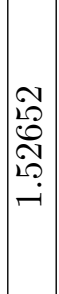 & 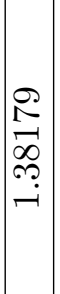 & 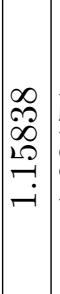 & 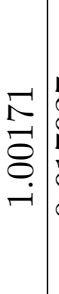 & 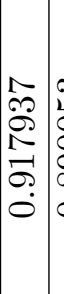 & 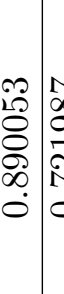 & 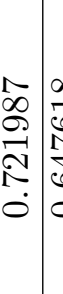 & 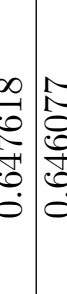 & 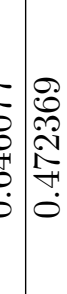 & 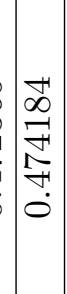 & 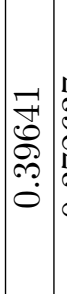 & 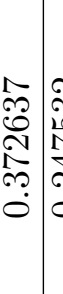 & 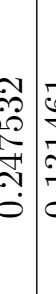 & 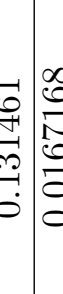 \\
\hline 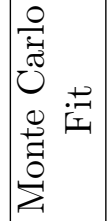 & $\mid \begin{array}{l}20 \\
\stackrel{20}{2} \\
2 \\
20 \\
-1 \\
-1\end{array}$ & $\begin{array}{c}+1 \\
N \\
\sim \\
\sim \\
\infty \\
-1 \\
-1\end{array}$ & $\begin{array}{l}0 \\
2 \\
1 \\
0 \\
0 \\
1 \\
-1 \\
-1\end{array}$ & $\begin{array}{l}\infty \\
0 \\
0 \\
0 \\
ٌ \\
0 \\
-1\end{array}$ & 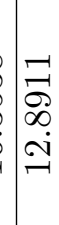 & 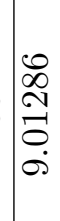 & $\begin{array}{l}\mathscr{2} \\
20 \\
20 \\
20 \\
0 \\
0\end{array}$ & $\begin{array}{l}\Re \\
0 \\
20 \\
0 \\
0 \\
10 \\
10\end{array}$ & $\left|\begin{array}{c}\mathfrak{y} \\
0 \\
1 \\
\infty \\
\sim \\
\sim \\
\forall\end{array}\right|$ & $\begin{array}{l}0 \\
\mathscr{2} \\
\stackrel{2}{D} \\
\stackrel{N}{\infty} \\
\dot{\infty}\end{array}$ & 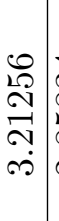 & 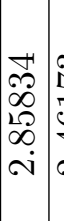 & 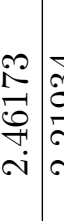 & 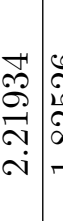 & 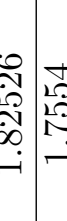 & 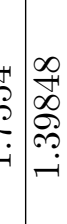 & 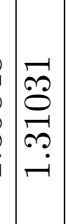 & 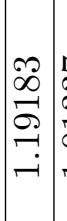 & 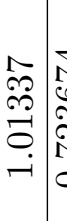 & 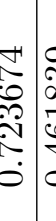 & 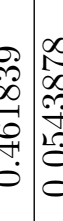 \\
\hline 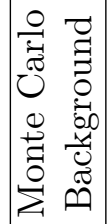 & $\mid \begin{array}{l}0 \\
20 \\
20 \\
10 \\
0 \\
-1 \\
\sim \\
ن\end{array}$ & $\begin{array}{l}13 \\
10 \\
0 \\
\infty \\
\infty \\
20\end{array}$ & 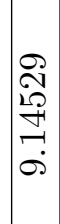 & $\begin{array}{l}\mathscr{2} \\
\infty \\
0 \\
0 \\
0 \\
0 \\
0\end{array}$ & 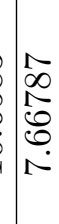 & 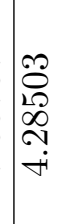 & $\begin{array}{l}\mathscr{L} \\
\infty \\
\infty \\
\infty \\
\approx \\
\sim \\
\sim\end{array}$ & 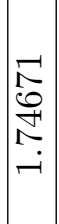 & 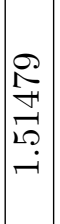 & $\begin{array}{l}\overrightarrow{1} \\
0 \\
\stackrel{+}{1} \\
\stackrel{-}{-}\end{array}$ & 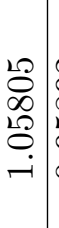 & 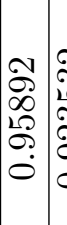 & 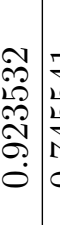 & 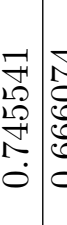 & 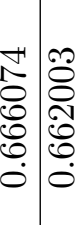 & 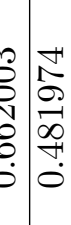 & \begin{tabular}{l}
0 \\
2 \\
0 \\
1 \\
0 \\
0 \\
\hdashline \\
0
\end{tabular} & 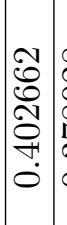 & 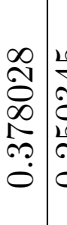 & 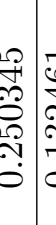 & 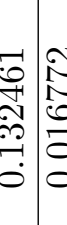 \\
\hline 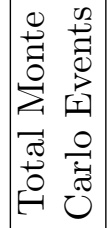 & 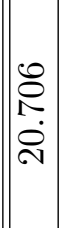 & 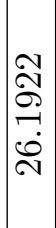 & 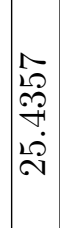 & 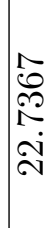 & 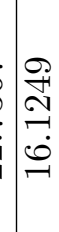 & 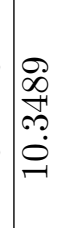 & 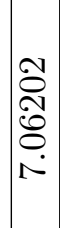 & $\begin{array}{l}0 \\
10 \\
0 \\
0 \\
0 \\
0 \\
10\end{array}$ & 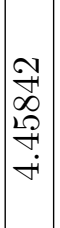 & 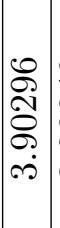 & 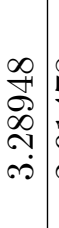 & 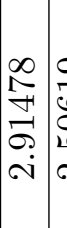 & 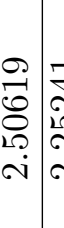 & 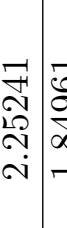 & 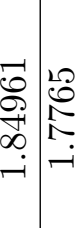 & 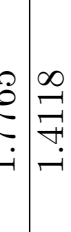 & 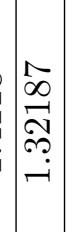 & 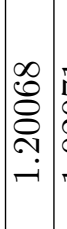 & 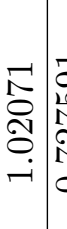 & 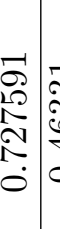 & 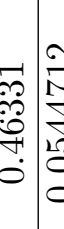 \\
\hline 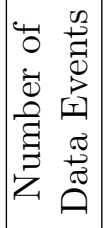 & $\stackrel{\oplus}{-1}$ & $\vec{\sim}$ & & $\infty$ & 0 & $\exists$ & 0 & 0 & $\infty$ & 0 & $\sim$ & $\infty 2$ & 206 & N & -10 & $\supset \sim$ & -1 & -1 & -12 & 20 & $\Rightarrow 25$ \\
\hline 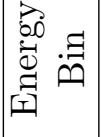 & $\stackrel{20}{0}$ & & & هُ & $\stackrel{L}{+}$ & $\mid \begin{array}{ll}2 \\
20\end{array}$ & $\begin{array}{l}10 \\
0 \\
0\end{array}$ & $\stackrel{20}{1}$ & $\begin{array}{l}20 \\
\infty \\
\infty\end{array}$ & $\begin{array}{c}10 \\
\infty\end{array}$ & $\begin{array}{l}L \rho \\
\stackrel{2}{0}\end{array}$ & 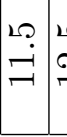 & $\begin{array}{l}\stackrel{2}{\mathrm{I}} \\
\stackrel{2}{I}\end{array}$ & 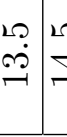 & 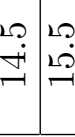 & 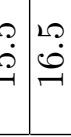 & 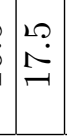 & $\left|\begin{array}{c}20 \\
\infty \\
\infty \\
-1\end{array}\right|$ & 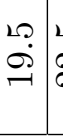 & & זִ \\
\hline
\end{tabular}

Table C.1: Composition of the Run I Neutral Current energy spectrum for both data and Monte Carlo. 


\begin{tabular}{|c|c|c|c|c|c|c|c|c|c|c|c|c|c|c|c|c|c|c|c|c|c|}
\hline 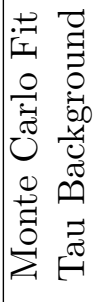 & $\mid \begin{array}{l} \\
0 \\
0 \\
0 \\
1 \\
0 \\
0 \\
8 \\
8 \\
0 \\
0 \\
0\end{array}$ & $\begin{array}{l}\infty \\
0 \\
\infty \\
0 \\
1 \\
20 \\
20 \\
0 \\
0 \\
0\end{array}$ & 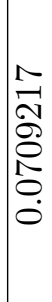 & $\begin{array}{l}3 \\
0 \\
0 \\
0 \\
10 \\
0 \\
0 \\
0 \\
0 \\
0\end{array}$ & 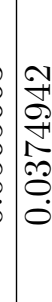 & 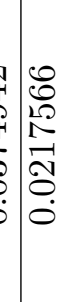 & $\left\{\begin{array}{l}\frac{m}{\vec{N}} \\
\stackrel{N}{N} \\
\frac{1}{0} \\
0 \\
0\end{array}\right.$ & $\begin{array}{l}0 \\
+ \\
0 \\
10 \\
+ \\
\\
0 \\
0 \\
0\end{array}$ & $\begin{array}{l}1 \\
0 \\
0 \\
0 \\
0 \\
0 \\
0 \\
0 \\
0\end{array}$ & $\begin{array}{l}\not{\infty} \\
0 \\
0 \\
0 \\
\infty \\
0 \\
0 \\
0 \\
0\end{array}$ & 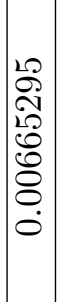 & $\begin{array}{l}0 \\
0 \\
0 \\
0 \\
0 \\
8 \\
0 \\
0 \\
0\end{array}$ & 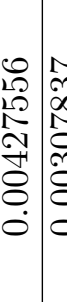 & 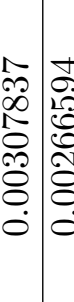 & 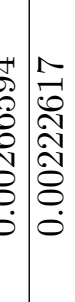 & $\begin{array}{l}\mathbb{1} \\
0 \\
0 \\
0 \\
0 \\
0 \\
0 \\
0\end{array}$ & & 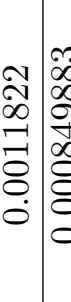 & 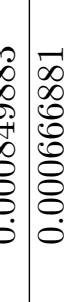 & 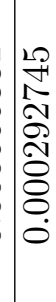 & 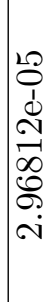 \\
\hline 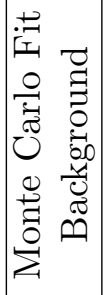 & $\mid \begin{array}{c}2-1 \\
20 \\
20 \\
20 \\
2+1 \\
20 \\
0 \\
0 \\
\end{array}$ & 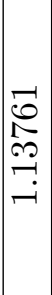 & $\begin{array}{l}\text { ㄱ. } \\
\text { o } \\
\text { N } \\
\stackrel{N}{N} \\
0\end{array}$ & 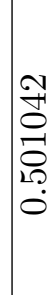 & $\begin{array}{l}\underset{\infty}{\infty} \\
\infty \\
10 \\
\sim \\
0 \\
0\end{array}$ & 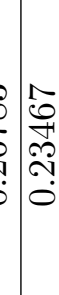 & 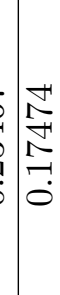 & 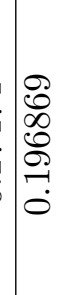 & 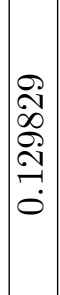 & $\begin{array}{l}\hat{1} \\
2 \\
\stackrel{2}{\mathcal{O}} \\
0 \\
\stackrel{-}{0} \\
0\end{array}$ & \begin{tabular}{|l|}
1 \\
2 \\
20 \\
2 \\
0 \\
0 \\
0 \\
0 \\
0 \\
0
\end{tabular} & \begin{tabular}{l}
\multicolumn{1}{c}{} \\
8 \\
0 \\
8 \\
0 \\
0 \\
0 \\
0
\end{tabular} & 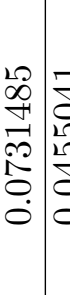 & 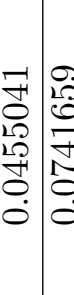 & 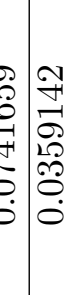 & 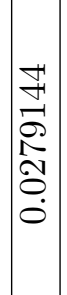 & & 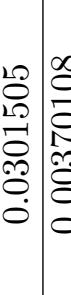 & 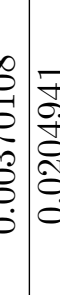 & $\begin{array}{l}2 \\
2 \\
2 \\
2 \\
2 \\
0 \\
0 \\
0 \\
0\end{array}$ & 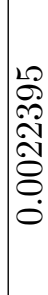 \\
\hline 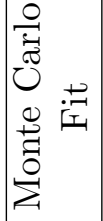 & 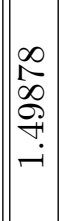 & 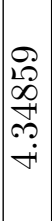 & 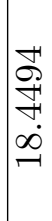 & 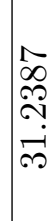 & $\left\{\begin{array}{l}\stackrel{\infty}{\infty} \\
\infty \\
\infty \\
\sim \\
\sim\end{array}\right.$ & 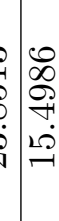 & 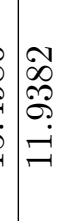 & 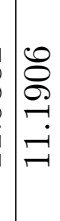 & 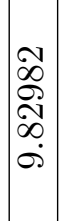 & 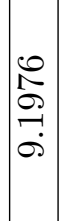 & $\begin{array}{c}\infty \\
\infty \\
\infty \\
\infty \\
\infty \\
\infty \\
\infty\end{array}$ & 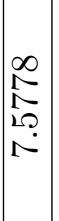 & 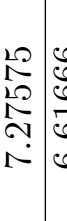 & 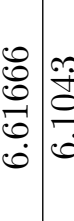 & 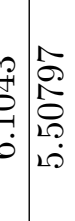 & 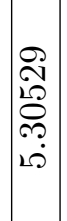 & 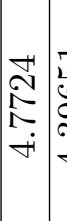 & 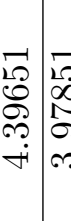 & 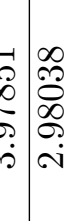 & $\left\{\begin{array}{l}0 \\
0 \\
0 \\
1 \\
\infty \\
-1 \\
-1\end{array}\right.$ & 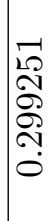 \\
\hline 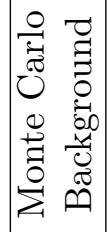 & $\mid \begin{array}{l}20 \\
20 \\
20 \\
0 \\
10 \\
0 \\
0 \\
0 \\
0\end{array}$ & 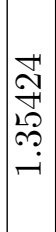 & $\begin{array}{l}0 \\
\mathscr{0} \\
\stackrel{0}{0} \\
0 \\
0 \\
0 \\
0\end{array}$ & 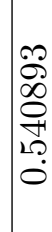 & 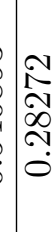 & 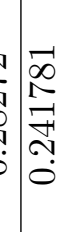 & 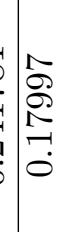 & 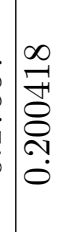 & $\mid \begin{array}{l}\vec{N} \\
\mathbb{N} \\
\vec{m} \\
\stackrel{\sim}{0} \\
\dot{0}\end{array}$ & $\begin{array}{l}20 \\
\stackrel{2}{0} \\
\stackrel{2}{2} \\
\stackrel{0}{0} \\
\stackrel{1}{0}\end{array}$ & \begin{tabular}{|l|}
1 \\
$\infty$ \\
$\infty$ \\
\hdashline \\
$\sigma$ \\
$\infty$ \\
$\infty$ \\
0 \\
0
\end{tabular} & $\begin{array}{l}0 \\
0 \\
0 \\
0 \\
0 \\
0 \\
0 \\
0\end{array}$ & 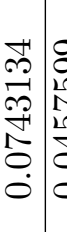 & 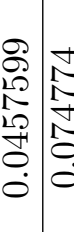 & 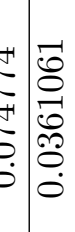 & $\begin{array}{l}0 \\
\stackrel{0}{0} \\
\stackrel{N}{0} \\
\infty \\
\mathbb{1} \\
\dot{0}\end{array}$ & 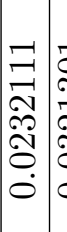 & 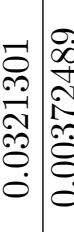 & 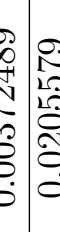 & $\left\{\begin{array}{l}\exists \\
\exists \\
\exists \\
\exists \\
0 \\
0 \\
0\end{array}\right.$ & 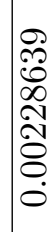 \\
\hline 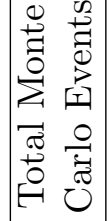 & $\mid \begin{array}{l}\hat{N} \\
i \\
i \\
0 \\
0 \\
0 \\
\sim \\
\sim\end{array}$ & 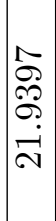 & 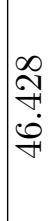 & 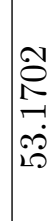 & 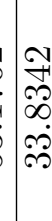 & 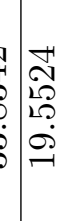 & $\left\{\begin{array}{l}10 \\
210 \\
2 \\
2 \\
20 \\
1\end{array}\right.$ & $\begin{array}{l}0 \\
20 \\
20 \\
\stackrel{20}{-1} \\
\end{array}$ & 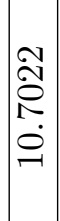 & 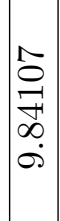 & $\mid \begin{array}{l}0 \\
2 \\
2 \\
2 \\
2 \\
12 \\
\infty \\
\infty\end{array}$ & $\begin{array}{l}0 \\
2 \\
\infty \\
D \\
0 \\
\\
\Gamma\end{array}$ & 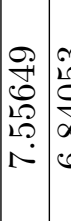 & 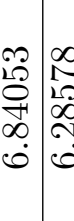 & 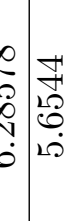 & 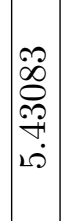 & 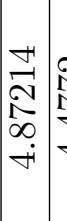 & 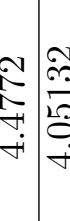 & 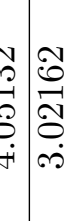 & 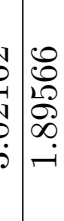 & 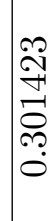 \\
\hline 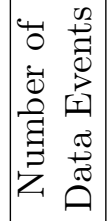 & $\sim$ & 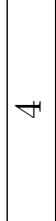 & $\stackrel{\infty}{-1}$ & $\infty$ & $\vec{N}$ & 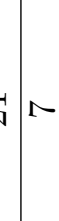 & $\exists$ & 0 & $\stackrel{-}{\ominus}$ & $\infty$ & 0 & os & 0 & $=0$. & $\infty$ & $\infty$ & $\begin{array}{lll}0 & 0\end{array}$ & 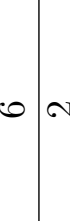 & $v=$ & 10 & ลึ \\
\hline 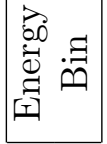 & $\stackrel{20}{0}$ & & & "ִ & $\stackrel{L}{\rightarrow}$ & \begin{tabular}{l|l} 
\\
\end{tabular} & $\mid \begin{array}{l}10 \\
0\end{array}$ & $\stackrel{L}{\stackrel{L}{\sim}}$ & $\begin{array}{l}20 \\
\infty \\
\infty\end{array}$ & $\stackrel{10}{\infty}$ & 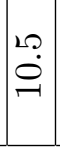 & $\mid$ & 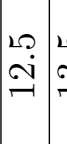 & 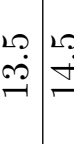 & 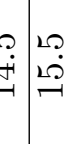 & $\mid \begin{array}{l}10 \\
0 \\
\bullet \\
-1\end{array}$ & 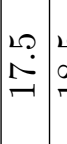 & 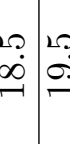 & 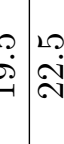 & 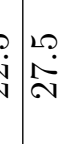 & 0 \\
\hline
\end{tabular}

Table C.2: Composition of the Run I Charged Current energy spectrum for both data and Monte Carlo. 


\begin{tabular}{|c|c|c|c|c|c|c|c|c|c|c|c|c|c|c|c|c|c|c|c|c|c|}
\hline 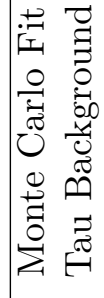 & $\mid \begin{array}{l} \\
20 \\
2 \\
20 \\
20 \\
i \\
20 \\
0 \\
0 \\
0 \\
0\end{array}$ & 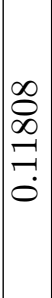 & 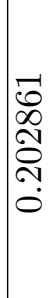 & 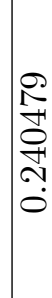 & 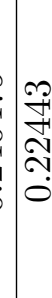 & 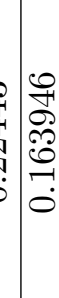 & 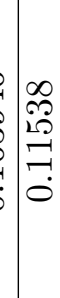 & 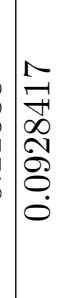 & 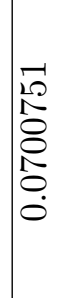 & 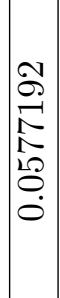 & 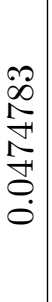 & 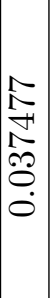 & 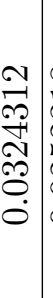 & 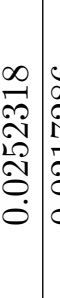 & 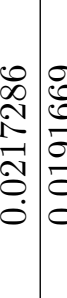 & 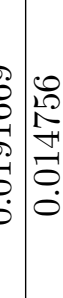 & 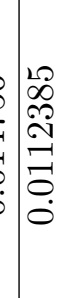 & $\mid \begin{array}{c}1 \\
0 \\
0 \\
0 \\
0 \\
0 \\
0\end{array}$ & 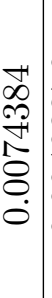 & 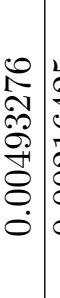 & 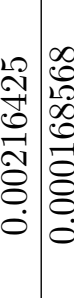 \\
\hline 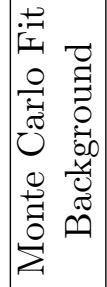 & $\mid \begin{array}{l}1 \\
1 \\
0 \\
0 \\
0 \\
0 \\
0 \\
0 \\
0\end{array}$ & 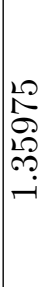 & $\begin{array}{l}10 \\
20 \\
0 \\
0 \\
20 \\
ن \\
0\end{array}$ & \begin{tabular}{l}
\multirow{N}{N}{} \\
\\
20 \\
0 \\
0
\end{tabular} & $\left\{\begin{array}{l}\overrightarrow{1} \\
1 \\
\infty \\
\sim \\
\sim \\
10\end{array}\right.$ & 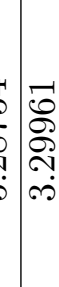 & 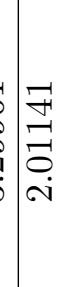 & 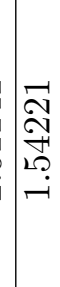 & 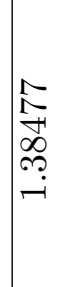 & 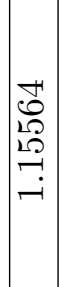 & 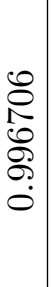 & $\begin{array}{l}0 \\
0 \\
0 \\
\cdots \\
\\
\sigma \\
0 \\
0\end{array}$ & $\begin{array}{l}1 \\
2 \\
2 \\
2 \\
\sim \\
\infty \\
0 \\
0 \\
0\end{array}$ & 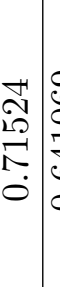 & 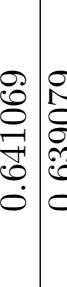 & 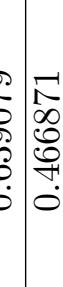 & 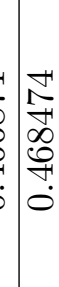 & 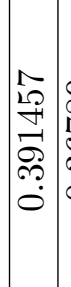 & 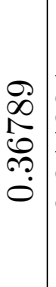 & 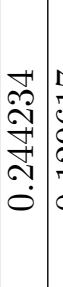 & 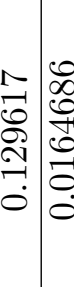 \\
\hline 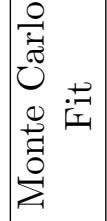 & $\mid \begin{array}{l}\mathscr{2} \\
2 \\
2 \\
20 \\
0 \\
0 \\
-1 \\
-1\end{array}$ & $\begin{array}{l}\infty \\
\mathscr{\sigma} \\
\sigma \\
\infty \\
-1\end{array}$ & $\begin{array}{l}20 \\
\mathscr{\Omega} \\
0 \\
+1 \\
\infty \\
-1 \\
-1\end{array}$ & 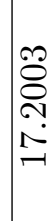 & $\left\{\begin{array}{l}N \\
N \\
N \\
\sim \\
-2 \\
-1\end{array}\right.$ & 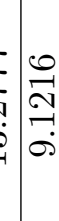 & 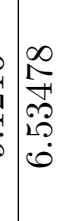 & $\begin{array}{l}\stackrel{P}{1} \\
\infty \\
20 \\
0 \\
\mathcal{L}\end{array}$ & 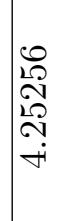 & $\begin{array}{l}0 \\
0 \\
0 \\
i 0 \\
10 \\
\infty \\
\infty\end{array}$ & 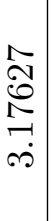 & 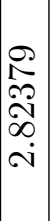 & 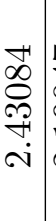 & 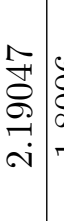 & 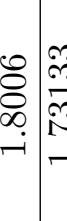 & 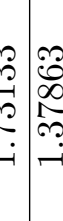 & 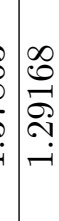 & 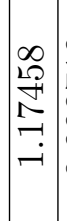 & $\begin{array}{l}\Omega \\
\tilde{D} \\
\infty \\
\mathscr{\Omega} \\
\Omega \\
0\end{array}$ & 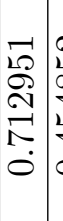 & 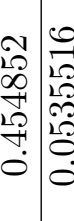 \\
\hline 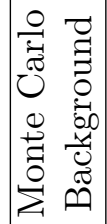 & 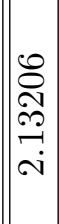 & $\begin{array}{l}\Re \\
0 \\
10 \\
0 \\
1 \\
10 \\
10\end{array}$ & $\begin{array}{l}1 \\
\infty \\
\tilde{0} \\
\tilde{\delta} \\
0 \\
0\end{array}$ & | & 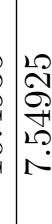 & 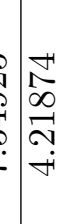 & 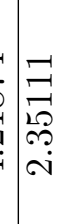 & 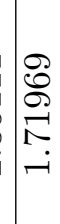 & & 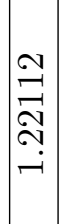 & $\mid \begin{array}{c}\infty \\
0 \\
ت \\
\\
0 \\
-1 \\
-1\end{array}$ & $\begin{array}{l}1 \\
\infty \\
0 \\
\stackrel{+}{1} \\
\\
0 \\
0\end{array}$ & 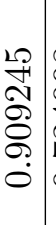 & 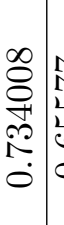 & 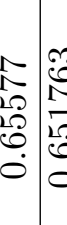 & 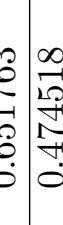 & 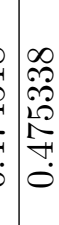 & 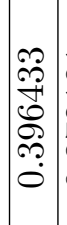 & $\begin{array}{l}\vec{\infty} \\
\stackrel{\sim}{N} \\
\stackrel{N}{o} \\
ٌ \\
0\end{array}$ & 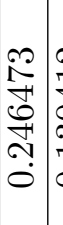 & 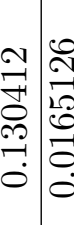 \\
\hline 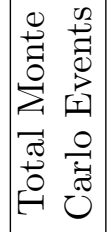 & 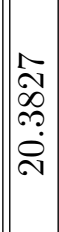 & 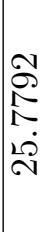 & 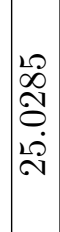 & 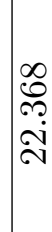 & {$\left[\begin{array}{l}\infty \\
\infty \\
2 \\
\infty \\
\infty \\
20 \\
-1\end{array}\right.$} & 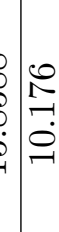 & 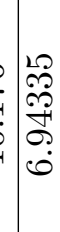 & 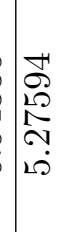 & 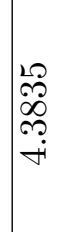 & 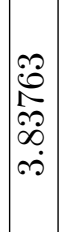 & 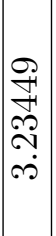 & $\begin{array}{l}\mathbb{H} \\
0 \\
0 \\
0 \\
0 \\
i\end{array}$ & 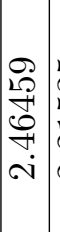 & 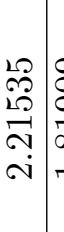 & 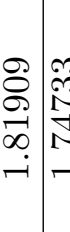 & 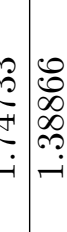 & 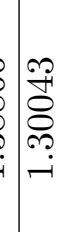 & 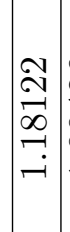 & 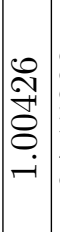 & $\begin{array}{l}\infty \\
\infty \\
\infty \\
\infty \\
10 \\
\\
0\end{array}$ & 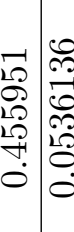 \\
\hline 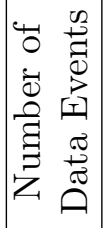 & $\stackrel{0}{0}$ & $\vec{N}$ & & & $\stackrel{0}{0}$ & $+\infty$ & $\infty \infty$ & 0 & 10 & 10 & $\sim$ & $\sim$ & $\infty$. & -1 & $\forall C$ & $b$ & 0 & $\sim$ & -1 & $\neg 0$ & 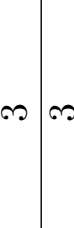 \\
\hline 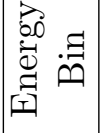 & $\stackrel{20}{0}$ & & & ספר & هُه & 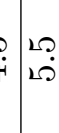 & $: \begin{array}{l}12 \\
0\end{array}$ & $\stackrel{L ?}{\Gamma}$ & $\underset{\infty}{20}$ & $\begin{array}{l}20 \\
\infty \\
\dot{5}\end{array}$ & $\begin{array}{l}20 \\
\stackrel{0}{0}\end{array}$ & $\left|\begin{array}{l}20 \\
\doteq \\
\hdashline\end{array}\right|$ & 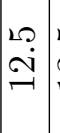 & 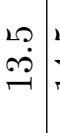 & 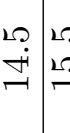 & 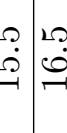 & 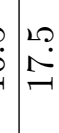 & $\begin{array}{l}20 \\
\infty \\
0 \\
-1\end{array}$ & $\mid \begin{array}{r}2 \rho \\
\ddot{\sigma}\end{array}$ & $\begin{array}{l}10 \\
\stackrel{N}{N} \\
\stackrel{N}{*}\end{array}$ & 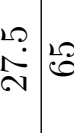 \\
\hline
\end{tabular}

Table C.3: Composition of the Run II Neutral Current energy spectrum for both data and Monte Carlo. 


\begin{tabular}{|c|c|c|c|c|c|c|c|c|c|c|c|c|c|c|c|c|c|c|c|c|c|}
\hline 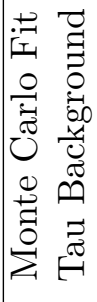 & 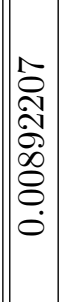 & $\begin{array}{l}20 \\
0 \\
1 \\
0 \\
0 \\
2 \\
0 \\
0 \\
0\end{array}$ & 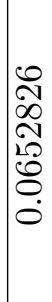 & 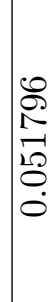 & $\left\{\begin{array}{l}10 \\
\stackrel{1}{I} \\
F \\
\hdashline \\
0 \\
0\end{array}\right.$ & 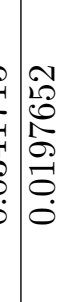 & $\begin{array}{l}0 \\
\stackrel{1}{1} \\
0 \\
0 \\
0 \\
0 \\
0 \\
0\end{array}$ & 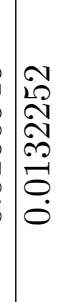 & $\begin{array}{l}0 \\
0 \\
0 \\
0 \\
0 \\
1 \\
0 \\
8 \\
0 \\
0\end{array}$ & $\begin{array}{l}N \\
1 \\
0 \\
0 \\
0 \\
0 \\
0 \\
0 \\
0\end{array}$ & 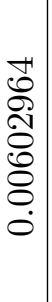 & $\mid \begin{array}{c}0 \\
1 \\
1 \\
1 \\
12 \\
0 \\
0 \\
0 \\
0 \\
0\end{array}$ & 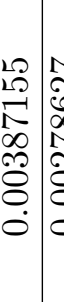 & 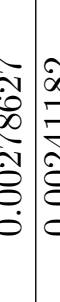 & 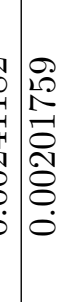 & \begin{tabular}{l}
20 \\
2 \\
0 \\
0 \\
20 \\
\hdashline \\
8 \\
0 \\
0
\end{tabular} & 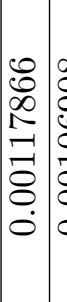 & 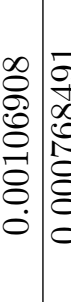 & 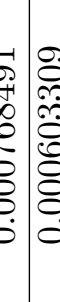 & & 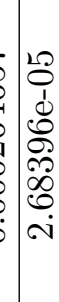 \\
\hline 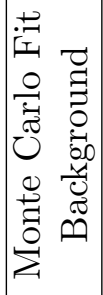 & $\left|\begin{array}{l}2 \\
1 \\
0 \\
0 \\
1 \\
20 \\
0 \\
0\end{array}\right|$ & $\begin{array}{l}\sim \\
i \\
\infty \\
\infty \\
-1 \\
-1 \\
-1\end{array}$ & $\begin{array}{l}10 \\
0 \\
0 \\
0 \\
8 \\
0 \\
0 \\
0\end{array}$ & 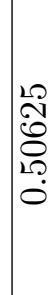 & $\left\{\begin{array}{l}\mathscr{\infty} \\
\tilde{\infty} \\
\infty \\
\sim \\
\sim \\
0\end{array}\right.$ & 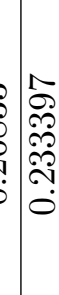 & 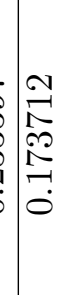 & 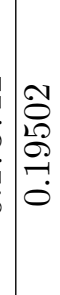 & 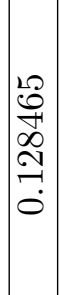 & 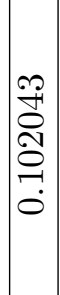 & 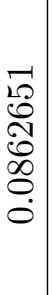 & 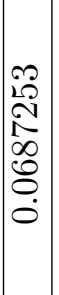 & 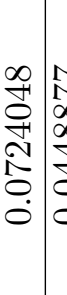 & 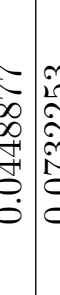 & 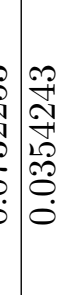 & 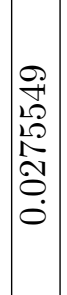 & & 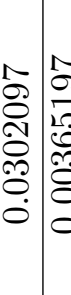 & 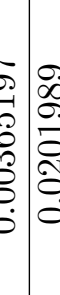 & & 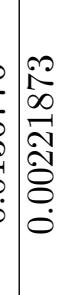 \\
\hline 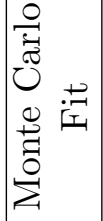 & 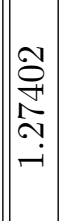 & 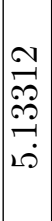 & $\begin{array}{l}\infty \\
\infty \\
\infty \\
\infty \\
\dot{A} \\
\end{array}$ & 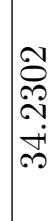 & 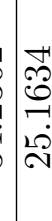 & 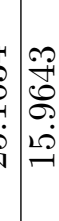 & 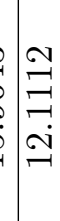 & 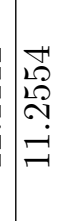 & $\begin{array}{l}\infty \\
\ddot{0} \\
\mathscr{0} \\
\mathscr{0} \\
\infty \\
\dot{0}\end{array}$ & 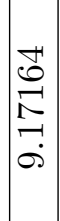 & 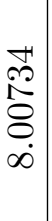 & 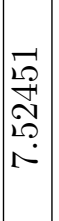 & 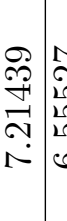 & 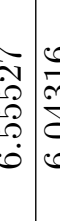 & 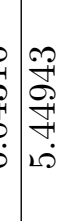 & 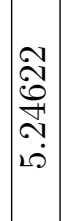 & 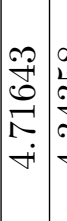 & 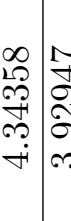 & 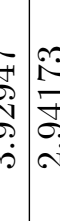 & & 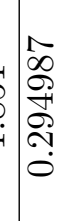 \\
\hline 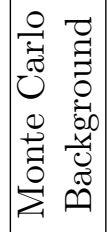 & $\mid \begin{array}{l}0 \\
2 \\
0 \\
0 \\
0 \\
0 \\
0 \\
0 \\
0\end{array}$ & 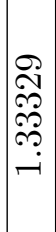 & $\begin{array}{l}2 \\
0 \\
10 \\
10 \\
10 \\
\infty \\
0 \\
0\end{array}$ & 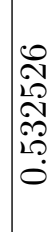 & 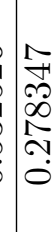 & 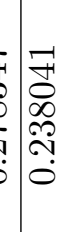 & $\left\{\begin{array}{l}\infty \\
\infty \\
N \\
N \\
\\
0\end{array}\right.$ & 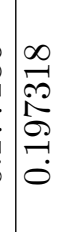 & 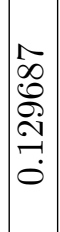 & $\begin{array}{l}\stackrel{+}{ } \\
\ddot{0} \\
\stackrel{-}{0} \\
0\end{array}$ & $\begin{array}{l}\infty \\
2 \\
10 \\
1 \\
1 \\
\infty \\
0 \\
0 \\
0\end{array}$ & $\mid$\begin{tabular}{c|}
0 \\
0 \\
0 \\
0 \\
0 \\
$\dot{0}$ \\
0 \\
0
\end{tabular} & 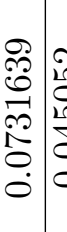 & 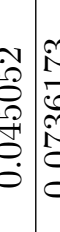 & 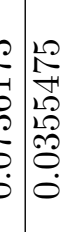 & 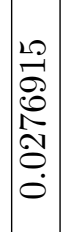 & 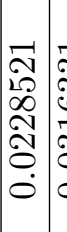 & 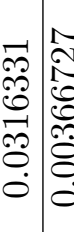 & 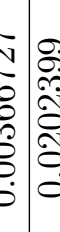 & $\left\{\begin{array}{l}\infty \\
\mathscr{N} \\
\delta \\
\infty \\
0 \\
0 \\
0 \\
0 \\
0\end{array}\right.$ & 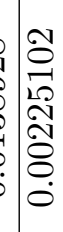 \\
\hline 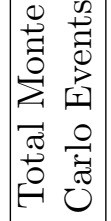 & $\mid \begin{array}{l}1 \\
2 \\
i \\
i \\
0 \\
0 \\
\sim \\
\sim\end{array}$ & 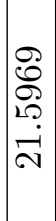 & 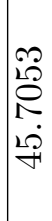 & 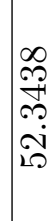 & 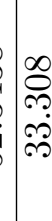 & 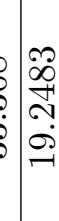 & 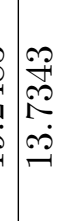 & 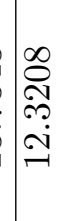 & $\begin{array}{l}\infty \\
1 \\
2 \\
0 \\
0 \\
0 \\
0 \\
-1\end{array}$ & $\begin{array}{l}10 \\
\infty \\
\infty \\
0 \\
0 \\
0\end{array}$ & 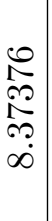 & 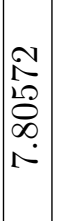 & 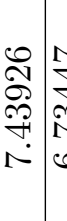 & 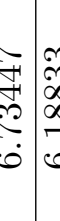 & 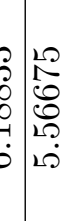 & 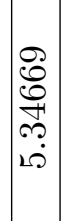 & 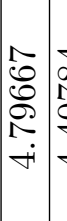 & 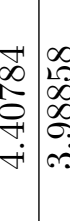 & 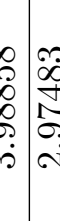 & & 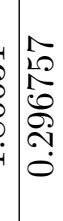 \\
\hline 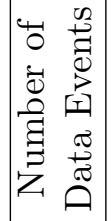 & -1 & 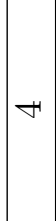 & $\stackrel{N}{N}$ & ब & & $\stackrel{\sim}{-}$ & $\infty$ & $\stackrel{\mathscr{I}}{-}$ & $\stackrel{m}{\rightarrow}$ & $\infty$ & $\infty$ & 0 & 0 & +10 & $\Rightarrow 0$ & $\stackrel{-}{\circ}$ & 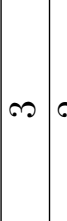 & $\checkmark 0$ & $0 \propto$ & 40 & סת \\
\hline 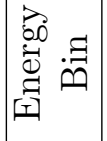 & {$\left[\begin{array}{l}20 \\
0\end{array}\right.$} & & $\stackrel{10}{10}$ & "ִ & $\stackrel{L}{\rightarrow}$ & \begin{tabular}{l|l} 
\\
\end{tabular} & $\mid \begin{array}{l}10 \\
0\end{array}$ & $\stackrel{L}{\sim}$ & $\begin{array}{l}20 \\
\infty\end{array}$ & $\begin{array}{l}10 \\
0\end{array}$ & $\mid \begin{array}{l}109 \\
\dot{0} \\
-1\end{array}$ & $\mid$ & 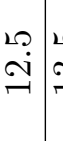 & هُ & 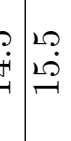 & $\mid \begin{array}{l}10 \\
0 \\
\bullet \\
-1\end{array}$ & م & 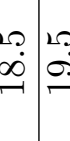 & 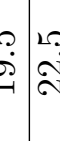 & is & 0 \\
\hline
\end{tabular}

Table C.4: Composition of the Run II Charged Current energy spectrum for both data and Monte Carlo. 


\begin{tabular}{|c|c|c|c|c|c|c|c|c|c|c|c|c|c|c|c|c|c|c|c|c|c|c|}
\hline 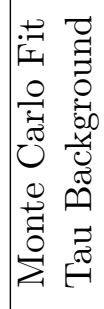 & 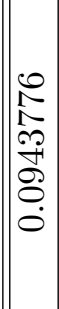 & \begin{tabular}{l}
$\stackrel{0}{0}$ \\
0 \\
0 \\
\multirow{2}{N}{} \\
0 \\
0
\end{tabular} & 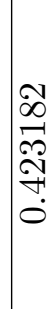 & 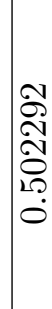 & $\left\{\begin{array}{l}20 \\
20 \\
20 \\
0 \\
0 \\
0 \\
0\end{array}\right.$ & 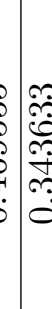 & 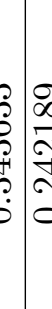 & & 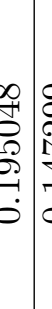 & 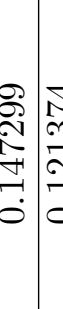 & 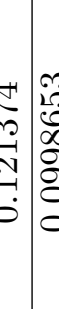 & 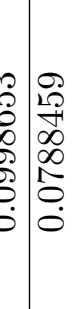 & $\begin{array}{l}\qquad \\
\stackrel{F}{7} \\
\mathbb{N} \\
0 \\
0 \\
0 \\
0\end{array}$ & 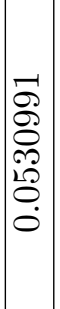 & 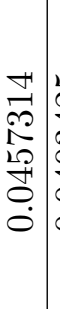 & 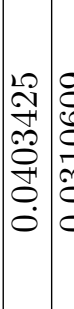 & 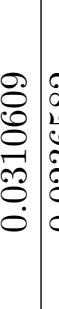 & 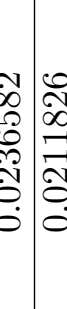 & 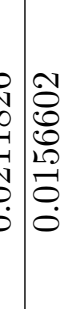 & $\left\{\begin{array}{l}\infty \\
2 \\
\infty \\
\infty \\
0 \\
0 \\
0 \\
0 \\
0 \\
0\end{array}\right.$ & & 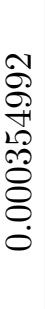 \\
\hline 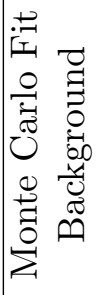 & $\mid \begin{array}{l}-1 \\
\\
2 \\
20 \\
0 \\
-1 \\
-1 \\
\end{array}$ & 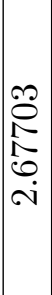 & 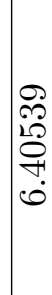 & 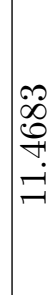 & 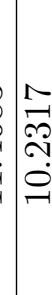 & $\begin{array}{l}-10 \\
1 \\
1 \\
0 \\
0 \\
0 \\
0 \\
0\end{array}$ & 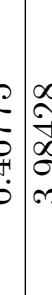 & & 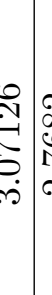 & 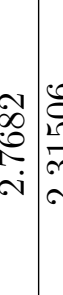 & 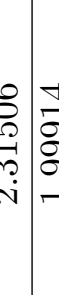 & 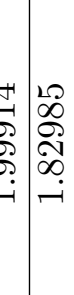 & 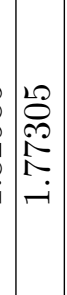 & 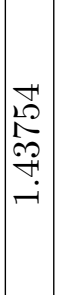 & \begin{tabular}{l|l}
$\mathscr{2}$ & 1 \\
2 & \\
$\infty$ & \\
$\infty$ & \\
- & \\
- &
\end{tabular} & 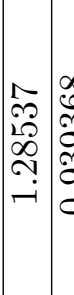 & 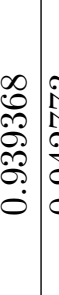 & 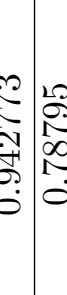 & 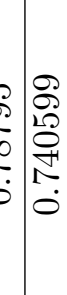 & 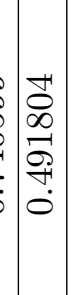 & & 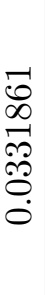 \\
\hline 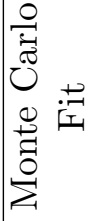 & 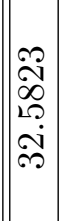 & $\underset{\substack{n \\
\infty}}{\stackrel{\infty}{\infty}}$ & 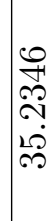 & 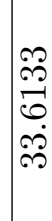 & 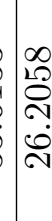 & 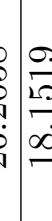 & 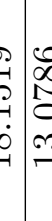 & 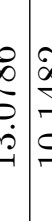 & \begin{tabular}{c|c}
1 \\
0 \\
$\vdots$ \\
$\vdots$ \\
$\vdots$ \\
$\vdots$ \\
\end{tabular} & 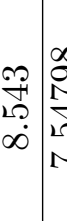 & 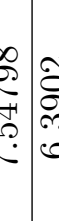 & 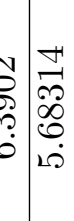 & 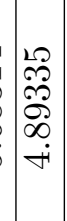 & 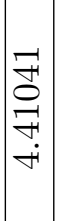 & 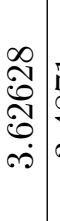 & 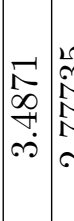 & 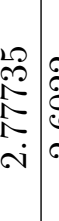 & 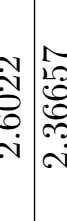 & 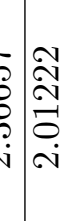 & 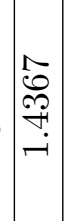 & $\begin{array}{l}2 \\
\overrightarrow{1} \\
0 \\
\sigma \\
0 \\
0\end{array}$ & $\begin{array}{l}\vec{F} \\
\stackrel{F}{2} \\
\text { s. } \\
\vdots \\
0\end{array}$ \\
\hline 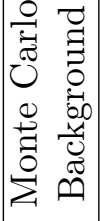 & 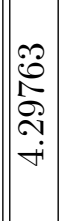 & 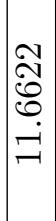 & 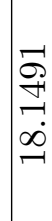 & 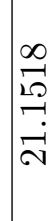 & $\begin{array}{l}\sqrt{\mathrm{N}} \\
\stackrel{2}{2} \\
20 \\
-2\end{array}$ & $\mid$\begin{tabular}{l}
1 \\
$\infty$ \\
$\infty$ \\
\hdashline \\
$\infty$ \\
$\infty$ \\
$\infty$ \\
$\infty$
\end{tabular} & 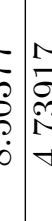 & 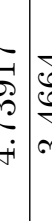 & 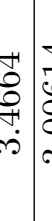 & 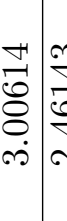 & 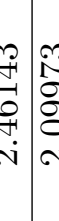 & 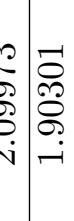 & 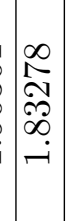 & 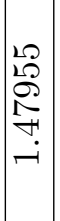 & 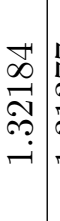 & 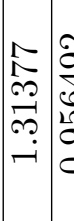 & 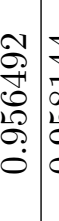 & 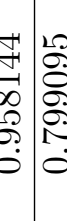 & 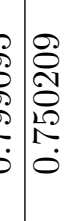 & $\mid \begin{array}{c}\infty \\
0 \\
0 \\
0 \\
\stackrel{5}{+} \\
\dot{0}\end{array}$ & 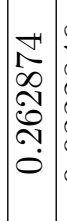 & 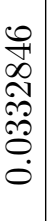 \\
\hline 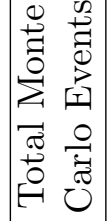 & $\mid \begin{array}{c}\infty \\
\infty \\
\infty \\
0 \\
0 \\
\dot{4} \\
\end{array}$ & 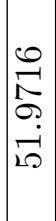 & 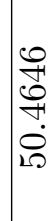 & 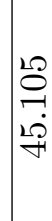 & 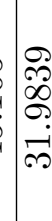 & 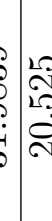 & 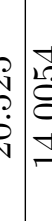 & 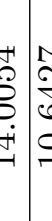 & 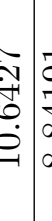 & 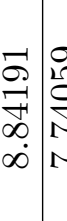 & 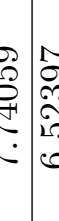 & 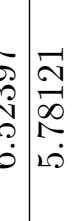 & 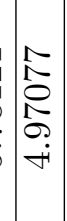 & 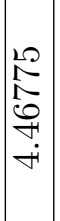 & \begin{tabular}{c|}
$\mathscr{B}$ \\
$\dot{0}$ \\
0 \\
0 \\
0 \\
$\dot{0}$ \\
$\dot{0}$
\end{tabular} & 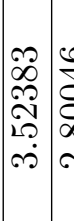 & 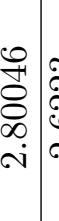 & 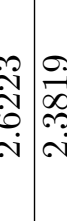 & 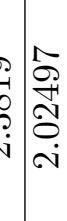 & $\mid \begin{array}{c}\stackrel{g}{+} \\
\stackrel{m}{\Psi} \\
\stackrel{\sim}{\sim}\end{array}$ & & $\begin{array}{l}12 \\
\infty \\
0 \\
0 \\
0 \\
-1 \\
0\end{array}$ \\
\hline 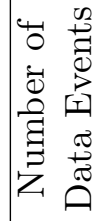 & $\stackrel{2}{2}$ & $\stackrel{\sim}{\not}$ & & 아 & $\stackrel{\mathscr{\Omega}}{\mathrm{N}}$ & 0 & $\rightarrow 1$ & & $\Omega_{1}^{0} 0$ & $\infty=$ & $\exists \mid$ & $\begin{array}{lll}4 & 10\end{array}$ & $\infty$ & $\infty$ & 10 & 0 & 7 & $-\infty$ & $\begin{array}{c}\curvearrowright \\
\end{array}$ & 0 & & $\infty$ \\
\hline 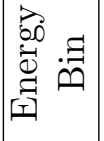 & : & & & ס & $\stackrel{\sim}{\sim}$ & 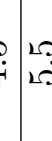 & $\begin{array}{llll} \\
\end{array}$ & \begin{tabular}{l|l} 
\\
$\stackrel{2}{0}$
\end{tabular} & ב & \begin{tabular}{c|c|c}
10 & 5 \\
$\infty$ & 0 & 0
\end{tabular} & 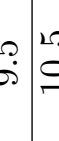 & 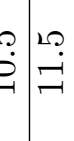 & 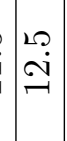 & $\mid$\begin{tabular}{l}
20 \\
$\stackrel{9}{9}$ \\
\hdashline
\end{tabular} & , & \begin{tabular}{l|l}
10 & 5 \\
10 & 5 \\
-1 & 5
\end{tabular} & | & 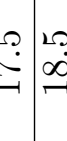 & 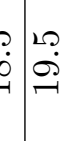 & $\mid \begin{array}{l}20 \\
\stackrel{i}{\mathrm{~N}}\end{array}$ & 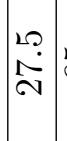 & 8 \\
\hline
\end{tabular}

Table C.5: Composition of the combined Run I and Run II Neutral Current energy spectrum for both data and Monte Carlo. 


\begin{tabular}{|c|c|c|c|c|c|c|c|c|c|c|c|c|c|c|c|c|c|c|c|c|c|}
\hline 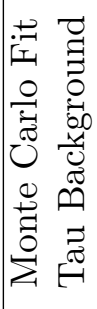 & $\mid \begin{array}{l} \\
0 \\
\dot{0} \\
0 \\
0 \\
\overrightarrow{0} \\
\dot{0} \\
0\end{array}$ & 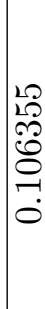 & \begin{tabular}{l}
$\infty$ \\
0 \\
$\infty$ \\
0 \\
0 \\
\hdashline \\
\hdashline \\
0
\end{tabular} & 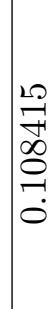 & 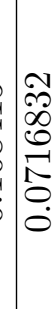 & 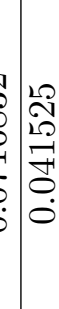 & \begin{tabular}{l}
$\infty$ \\
$\infty$ \\
$\infty$ \\
2 \\
\hdashline \\
0 \\
0 \\
0
\end{tabular} & 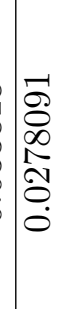 & $\begin{array}{l}20 \\
2 \\
0 \\
2 \\
2 \\
0 \\
0 \\
0\end{array}$ & $\begin{array}{c}1 \\
\infty \\
0 \\
0 \\
0 \\
0 \\
0 \\
0 \\
0\end{array}$ & $\mid \begin{array}{c}0 \\
\overrightarrow{0} \\
0 \\
\stackrel{0}{0} \\
ت \\
0 \\
0 \\
0\end{array}$ & 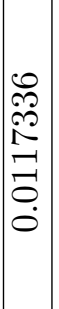 & 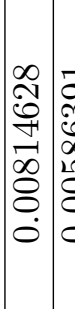 & 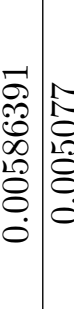 & 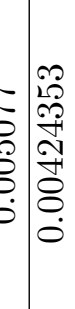 & 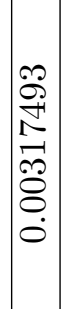 & 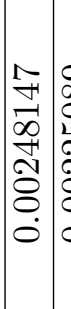 & 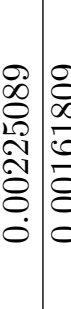 & 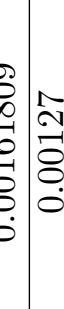 & 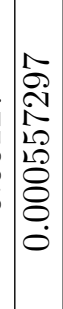 & 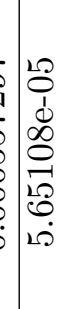 \\
\hline 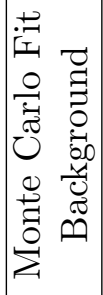 & 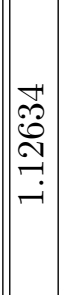 & 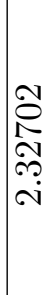 & $\begin{array}{l}20 \\
10 \\
01 \\
0 \\
0 \\
-1\end{array}$ & 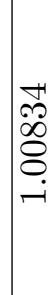 & 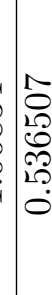 & 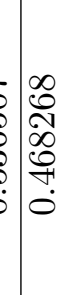 & 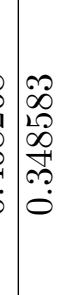 & 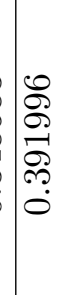 & 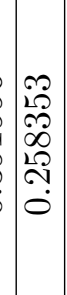 & 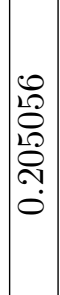 & 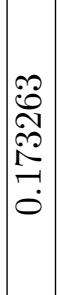 & 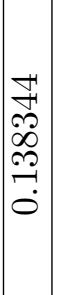 & 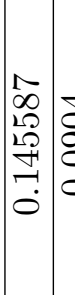 & 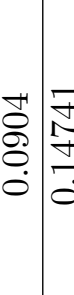 & 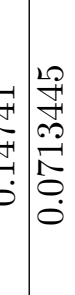 & $\begin{array}{l}0 \\
2 \\
2 \\
21 \\
20 \\
0 \\
0 \\
0\end{array}$ & 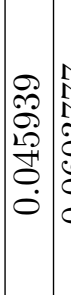 & 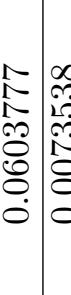 & 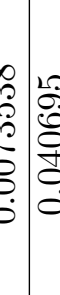 & & 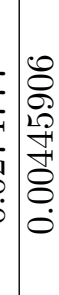 \\
\hline 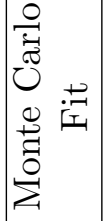 & $\mid \begin{array}{l}2 \\
2 \\
0 \\
0 \\
1 \\
i v \\
\sim\end{array}$ & 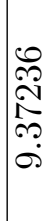 & 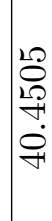 & 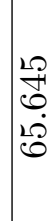 & 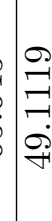 & 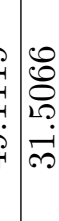 & 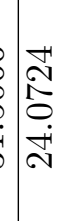 & 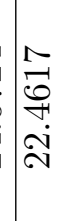 & $\begin{array}{l}\infty \\
0 \\
0 \\
0 \\
0 \\
9 \\
-9\end{array}$ & $\left|\begin{array}{l}\Gamma \\
N \\
\sim \\
\infty \\
\infty \\
\sim\end{array}\right|$ & $\mid \begin{array}{c}\overrightarrow{1} \\
\overline{0} \\
0 \\
\dot{0} \\
-1\end{array}$ & $\begin{array}{c}1 \\
0 \\
0 \\
-1 \\
10 \\
-1 \\
-1\end{array}$ & 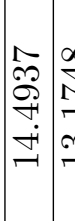 & 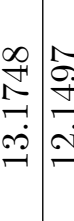 & 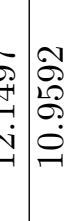 & $\begin{array}{l}\overrightarrow{0} \\
i 0 \\
0 \\
0 \\
0 \\
0 \\
=\end{array}$ & 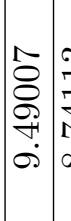 & 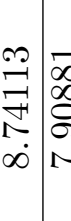 & 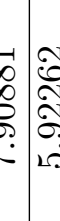 & & 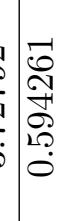 \\
\hline 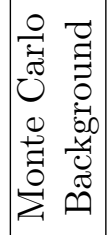 & 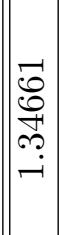 & $\begin{array}{l}\sim \\
1 \\
1 \\
\infty \\
0 \\
0 \\
\sim \\
\sim\end{array}$ & 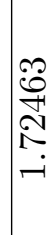 & $\underset{\mathcal{F}}{\mathcal{H}}$ & 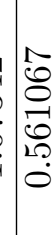 & 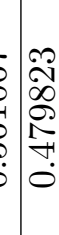 & $\mid \begin{array}{l}0 \\
20 \\
1 \\
1 \\
20 \\
2 \\
0 \\
0\end{array}$ & 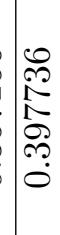 & 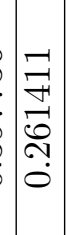 & 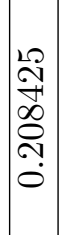 & $\mid$\begin{tabular}{l}
$\mathscr{D}$ \\
$\mathscr{D}$ \\
$\infty$ \\
0 \\
2 \\
\hdashline \\
0 \\
0
\end{tabular} & 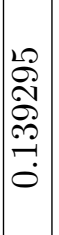 & 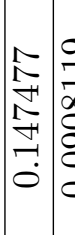 & 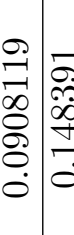 & 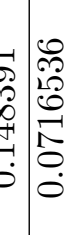 & $\begin{array}{l}\infty \\
0 \\
\infty \\
20 \\
20 \\
0 \\
0\end{array}$ & 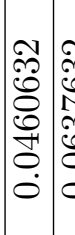 & 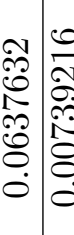 & 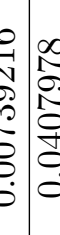 & 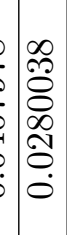 & 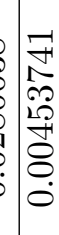 \\
\hline 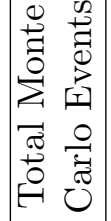 & 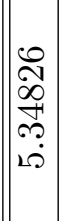 & 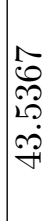 & 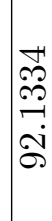 & ف․ & $\underset{\stackrel{N}{\sim}}{\stackrel{\sim}{\sim}}$ & 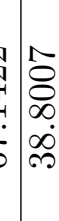 & 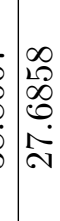 & 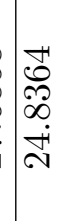 & 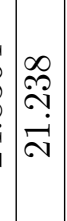 & 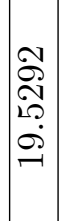 & $\mid \begin{array}{l}0 \\
\mathscr{2} \\
\infty \\
0 \\
0 \\
-1\end{array}$ & $\mid \begin{array}{l}0 \\
21 \\
2 \\
20 \\
10 \\
-1\end{array}$ & 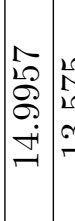 & 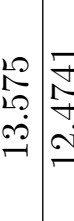 & 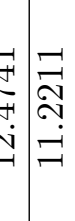 & $\left|\begin{array}{l}10 \\
1 \\
1 \\
1 \\
0 \\
-1\end{array}\right|$ & \begin{tabular}{|l|l}
$\infty$ & \multicolumn{1}{c}{} \\
$\infty$ & 2 \\
0 & 1 \\
0 & $\alpha$ \\
0 & $\alpha$ \\
0 & $\alpha$ \\
& $\alpha$
\end{tabular} & 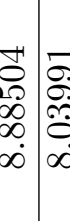 & 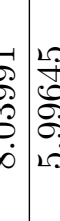 & & $\begin{array}{l}\infty \\
-1 \\
\infty \\
0 \\
0 \\
0 \\
0 \\
0\end{array}$ \\
\hline 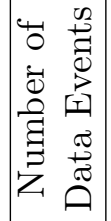 & $\infty$ & $\infty$ & $\stackrel{ }{\not}$ & $\frac{1}{6}$ & 8 & $\stackrel{\Omega}{=}$ & ন & $\stackrel{\infty}{=}$ & $\stackrel{9}{\sim}$ & $\stackrel{ }{\sim}$ & $\sigma$ & $\stackrel{20}{-1}$ & $\stackrel{0}{-1}$ & 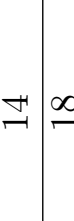 & $\begin{array}{lll}0 & 0 \\
-1 & 0\end{array}$ & $\underset{\infty}{\infty}$ & $\begin{array}{ll}0 & 0\end{array}$ & $\infty \mid \infty$ & $\circ:$ & & $\begin{array}{l}\infty \\
10\end{array}$ \\
\hline 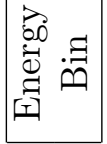 & : & & $\stackrel{10}{i}$ & סִ & $\stackrel{\sim}{\sim}$ & \begin{tabular}{l|l} 
\\
\end{tabular} & $\mid \begin{array}{l}10 \\
0\end{array}$ & $\stackrel{L}{\stackrel{L}{\sim}}$ & 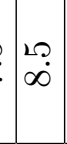 & $\stackrel{10}{\infty}$ & 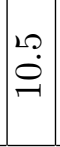 & $\mid$ & 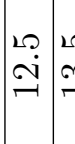 & 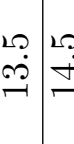 & 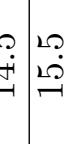 & $\mid \begin{array}{l}10 \\
0 \\
\bullet \\
-1\end{array}$ & 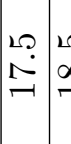 & 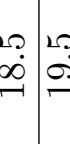 & 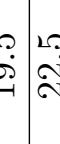 & $\mid \begin{array}{ll}n \\
\stackrel{n}{N}\end{array}$ & ס \\
\hline
\end{tabular}

Table C.6: Composition of the combined Run I and Run II Charged Current energy spectrum for both data and Monte Carlo. 


\section{Bibliography}

[1] W. Pauli, Letter to a physicists' gathering at Tubingen. December 4, 1930, reprinted in Wolfgang Pauli, Collected Scientific Papers, edited by R. Kronig and V. Weisskopf, vol. 2, p. 1313, New York, Interscience, 1964.

[2] E. Fermi, An attempt of a theory of beta radiation, Z. Phys. 88 (1934) 161-177.

[3] C.L. Cowan and F. Reines, Detection of the free neutrino, Phys. Rev. 92, 830831 (1953).

[4] The LEP Collaboration and the LEP Electroweak Working Group, as reported by J. Dress at the XX International Symposium on Lepton and Photon Interactions at High Energy, Rome, Italy (July 2001).

[5] G. Danby et al., Observation of high-energy neutrino reactions and the existence of two kinds of neutrinos, Phys Rev. Lett. 9 (1962) 36-44.

[6] DONUT Collaboratino, K. Kodama et al., Observation of tau-neutrino interactions, Phys. Lett. B. 504 (2001) 218-224.

[7] Particle Data Group 2006.

[8] Lane, J. Homer 1869, Amer. J. Sci., 2nd ser., 50, 57.

[9] von Weizscker, C. F., 1937, Phys. Z., 38, 176. 
[10] Gamow, G. 1938, Phys. Rev., 53, 595.

[11] Bethe, H. 1939, Phys. Rev., 55, 434

[12] Oke, J. B. 1950, J. Roy. Astron. Soc. Canada, 44, 135

[13] J. N. Bahcall, M. H. Pinsonneault and S. Basu, Solar Models: Current epoch and time dependences, neutrinos, and heliuseismological properties, Astrophys. J. 555 (2001) 990-1012.

[14] B. Pontecorvo, Mesonium And Antimesonium, Sov. Phys. JETP 6, 429 (1957).

[15] B. Pontecorve, Inverse Beta Processes And Nonconservation Of Lepton Charge, Sov. Phys. JETP 7, 172. (1958)

[16] B. T. Cleveland, et al., Measurement of the solar electron neutrino flux with the Homestake chlorine detector, Astrophys. J. 496 (1998) 505-526.

[17] Constraints on neutrino oscillation parameters from the Kamiokande-II solar neutrino data, Hirata, K. S. et al. (Kamiokande), Phys. Rev. Lett. 65 (1990) 1301-1304.

[18] Measurement of the Solar Neutrino Capture Rate by the Russian-American Gallium Solar Neutrino Experiment During One Half of the 22-Year Cycle of Solar Activity, Abdurashitov, J. N. et al. (SAGE), J. Exp. Theor. Phys. 95 (2002) 181-193, arXiv:astro-ph/0204245.

[19] GALLEX solar neutrino observations: Results for GALLEX IV, Hampel, W. et al. (GALLEX), Phys. Lett. B447 (1999)

[20] Solar neutrino measurements in Super-Kamiokande-I, Hosaka, J. et al. (SuperKamkiokande), Phys. Rev. D73 (2006) 112001, arXiv:hep-ex/0508053. 
[21] Y. Fukuda, et al., Evidence for oscillations of atmospheric neutrinos, Phys. Rev. Lett. 81, 1562-1567 (1998).

[22] Q.R. Ahmad, et al. Direct evidence for neutrino flavor transformation from neutral-current interactions in the sudbury neutrino observatory, Phys. Rev. Lett. 89 (2002) 011301.

[23] F. Reines, H.W. Sobel, and E. Pasierb. Evidence for Neutrino Instability. Phys. Rev. Lett. 45, 1307-1311 (1980).

[24] Indication for neutrino oscillation from a high statistics experiment at the bugey reactor, Cavaignac, J. F. et al. (Bugey), Phys. Lett. B148 (1984) 387-394.

[25] Study of reactor anti-neutrino interaction with proton at Bugey nuclear power plant, Declais, Y. et al. (Bugey), Phys. Lett. B338 (1994) 383-389.

[26] Neutrino oscillation experiments at the Gosgen nuclear power reactor, Zacek, G. et al. (CalTech-SIN-TUM), Phys. Rev. D34 (1986) 2621-2636.

[27] M. Apollonio et al., Search for neutrino oscillations on a long base-line at the CHOOZ nuclear power station, Eur. Phys. Nucl. Phys. 48 (2002) 113-121.

[28] G. Fogli, Neutrino parameters, in Next Generation of Nucleon Decay and Neutrino Detectors 2005 (NNN05), Aussois, Savoie, France 2005.

[29] KamLAND Collaboration, T. Araki et al., Measurement of neutrino oscillation with KamLAND: Evidence of spectral distortion, hep-ex/0406035.

[30] K2K Collaboration, E. Aliu et al., Evidence for muon oscillation in an accelerator-based experiment, hep-ex/0411038.

[31] The MINOS Collaboration. arXiv:0708.1495v2. 
[32] Z. Maki, M. Nakagawa and S. Sakata, Remarks on the unified model of elementary particles, Prog. Theor. Phys. 28 (1962) 870.

[33] B. Kayser, On the Quantum Mechanics of Neutrino Oscillation, Phys. Rev. D24, 110 (1981).

[34] B. Pontecorvo, JETP, 53, 1717 (1967)

[35] P. Minkowski, Phys. Lett B67, 421 (1977).

[36] A. de Gouvea, Phys. Rev. D 72, 033005 (2005).

[37] G. C. McLaughlin, J. M. Fetter, A. B. Balantekin and G. M. Fuller, Phys. rev. C 59, 2873 (1999); D. O. Caldwell, G. M. Fuller and Y. Z. Qian, Phys. Rev. D 61, 123005 (2000); J. Fetter, G. C. McLaughlin, A. B. Balantekin and G. M. Fuller, Astropart. Phys. 18, 433 (2003).

[38] A. Kusenko and G. Segre, Phys. Lett B 396, 197 (1997); A. Kusenko and G. Segre, Phys. Rev. D 59, 061302 (1999).

[39] G. M. Fuller, A. Kusenko, I. Mocioiu, and S. Pascoli, Phys. Rev. D 68, 103002 (2003); M. Barkovich, J.C. D’Olivo and R. Montemayor, Phys. Rev. D 70, 043005 (2004).

[40] A. Kusenko, Int. J. Mod. Phys. D 13, 2065 (2004).

[41] S. Dodelson and L. M. Widrow, Phys. Rev. Lett. 72, 17 (1994).

[42] K. Abazajian, G. M. Fuller and M. Patel, Phys. Rev. D 64, 023501 (2001); A. D. Dolgov and S. H. Hansen, Astropart. Phys. 16339 (2002).

[43] X. d. Shi and G. M. Fuller, Phys. Rev. Lett. 822832 (1999).

[44] T. Asaka, S. Blanchet and M. Shaposhnikov, Phys. Lett. B 631, 151 (2005). 
[45] F. Munyanesa, P. L. Biermann, Astron and Astrophys., 436, 805 (2005).

[46] P.L. Biermann and A. Kusenko, Phys. Rev. Lett. 96, 091301 (2006); M. Mapelli, A. Ferrara and E. Pierpaoli, Mon. Not. Roy. Astron. Soc. 369, 1719 (2006); J. Stasielak, P. L. Biermann and A. Kusenko, Astrophys. J. 654, 290 (2007); E. Ripamonti, M. Mapelli and A. Ferrara, Mon. Not. Roy. Astron. Soc. 375, 1399 (2007).

[47] P. Candelas and S. Kalara, Nucl. Phys. B 298, 357 (1988). D. Gepner, Nucl. Phys. B 311, 191 (1988).

[48] O. J. Eyton-Williams and S. F. King, JHEP 0506, 040 (2005).

[49] E. A. Mirabelli and M. Schmaltz, Phys. Rev. D 61, 113011 (2000).

[50] M. Fukugita and T. Yanagida, Phys. Lett. B 174 (1986) 45.

[51] E. K. Akhmedov, V. A. Rubakov and A. Y. Smirnov, Phys. Rev. Lett. 81, 1359 (1998); T. Asaka and M. Shaposhnikov, Phys. Lett. B 620, 17 (2005).

[52] R. Barbieri and A. Dolgov, Phys. Lett. B 237, 440 (1990); K. Kainulainen, Phys. Lett. B 244, 191 (1990); K. Enquist, K. Kainulainen and M. J. Thomson, Nucl. Phys. B 373, 498 (1992); D. P. Kirilova and M. V. Chizhov, Phys. Rev. D 58, 073004 (1998); A. D. Dolgov, Phys. Lett B. 506, 7 (2001); M. Cirelli, G. Marandella, A. Strumia and F. Vissani, Nucl. Phys. B 708, 215 (2005).

[53] C. Y. Cardall and G. M. Fuller, Phys. Rev. D 541260 (1996); X. D. Shi and G. M. Fuller, Phys. Rev. D 59, 063006 (1999).

[54] A. Y. Smirnov and R. Zukanovich Funchal, Phys. Rev. D 74, 013001 (2006).

[55] C. Athanassopoulos et al. [LSND Collaboration], Phys. Rev. Lett. 77, 3082 (1996); Phys. Rev. C 58, 2489 (1998); Phys. Rev. Lett. 81, 1774 (1998). 
[56] M. Sorel, J. M. Conrad, and M. Shaevitz, Phys. Rev. D 70, 073004 (2004).

[57] S. Palomares-Ruiz, S. Pascoli and T. Schweta, JHEP 0509, 048 (2005) [arXiv:hep-ph/0505216]

[58] http://www-boone.fnal.gov/publicpages/detector_tdr.ps.gz

[59] MiniBooNE Collaboration. [arXiv:hep-ex/0704.1500v3]

[60] B. Armbruster et al., Phys. Rev. D 65, 112001 (2002).

[61] B. Achkar et al. Nucl. Phys. B434, 503 (1995).

[62] arXiv:hep-exp/0009001

[63] Shiozawa, M. for the Super-K Collaboration, talk presented at the 20th International Cosmic Ray Conference, May 2002, Munich, Germany.

[64] Fogli et al., PRD 63 (053008) 2001.

[65] R. E. Shrock, Phys. Rev. D 241232 (1981).

[66] S. R. Elliott and P. Vogel, Ann. Rev. Nucl. Part. Sci. 52, 115 (2002).

[67] G. Finocchiaro and R. E. Shrock, Phys. Rev. D 46, 888 (1992); F. Bezrukov and M. Shaposhnikov, Phys. Rev. D 74, 053005 (2007).

[68] A. Kusenko, S. Pascoli and D. Semikoz, JHEP 0511, 028 (2005).

[69] A. Kusenko [arXiv:hep-ph/07031161]

[70] Y. Z. Chu and M. Cirelli, Phys. Rev. D 74, 085015 (2006).

[71] G. Gelmini, S. Palomares-Ruiz and S. Pascoli, Phys. Rev. Lett. 93, 081302 (2004).

[72] M. Shaposhnikov and I. Tkachev, Phys. Lett. B 639, 414 (2006). 
[73] A. Kusenko, Phys. Rev. Lett. 97, 241301 (2006).

[74] K. Abazajian, G. M. Fuller and W. H. Tucker, Astrophys. J. 562, 593 (2001); A. Boyarsky, A. Neronov, O. Ruchayskiy and M. Shaposhnikov, JETP Lett. 83, 133 (2006).

[75] M. Viel, et al. Phys. Rev. D. 71, 063534 (2005); M. Viel, J. Lesgourgues, M. G. Haehnelt, S. Matarrese and A. Riotto, Phys. Rev. Lett. 97, 071301 (2006).

[76] P. B. Pal and L. Wolfenstein, Phys. Rev. D 25, 766 (1982).

[77] K. Abazajian, Phys. Rev. D. 73063506 (2006).

[78] M. Valdes, A. Ferrara, M. Mapelli and E. Ripamonti, ArXiv:astro-ph/0701301

[79] A. Kusenko and G. Segre, Phys. Lett B 396, 197 (1997); A. Kusenko and G. Segre, Phys. Rev. D 59, 061302 (1999).

[80] G. M. Fuller, A. Kusenko, I. Mocioiu, and S. Pascoli, Phys. Rev. D 68, 103002 (2003); M. Barkovich, J.C. D’Olivo and R. Montemayor, Phys. Rev. D 70, 043005 (2004).

[81] A. Kusenko, Int J. Mod. Phys. D 13, 2065 (2004).

[82] A. Kusenko, G. Segre and A. Vilenkin, Phys. Lett. B 437, 359 (1998).

[83] J. Hylen et al., "NuMI Facility Technical Design Report," Fermilab-TM-2018, Sept., 1997.

[84] The MINOS Collaboration, Fermilab NuMI-337, October 1998, S. Wojcicki, spokesperson.

[85] Zwaska, Robert M. "Accelerator systems and instrumentation for the NuMI neutrino beam". PhD Thesis, University of Texas at Austin, December 2005. 
[86] N. Tagg et al., Performace of Hamamatsu 64-anode photomultipliers for use with wavelength-shifting optical fibers, Nucl. Instrum. Meth. A539, 668 (2005).

[87] K. Lang et al., Characterization of 1600 Hamamatsu 64-anode photomultipliers for the MINOS Far detector, Nucl. Intrum. Meth. A545, 852 (2005).

[88] T. Cundiff et al., The MINOS Near detector front end electronics, IEEE Trans. Mucl. Sci 53, 1347 (2006).

[89] Anatael Cabrera, Jeffrey Hartnell and the MINOS Collaboration. Nucl. Phys. B 143, 533 (2005).

[90] Kordosky, Michael A. "Hadronic interactions in the MINOS detectors". PhD Thesis, University of Texas at Austin, August 2004.

[91] Vahle, Patricia L. "Electromagnetic interactions in the MINOS detectors". PhD Thesis, University of Texas at Austin, August 2004.

[92] Cabrera, Anatael. "Systematic Comparison of the MINOS Near and Far Detector Readout Systems". PhD Thesis, University of Oxford, May 2005.

[93] H. Gallagher, Nucl. Phys. Proc. Suppl. 112: 188-194, 2002.

[94] D. Rein and L. M. Sehgal, Ann. Phys. 133, 79 (1981).

[95] A. Bodek and U. K. Yang, AIP Conf. Proc. 721, 358 (2004).

[96] Dierckxsens, M. "Accessing Beam Monitoring for Physics Analyses", MINOSdoc-1486, January 30, 2006.

[97] Indurthy, D. "Beam Monitoring Devices in the NuMI Beamline", Master's Thesis, University of Texas at Austin.

[98] Raufer, T. MINOS Internal Document 2588. 
[99] Plot provided by Sacha Kopp.

[100] Petyt, D. The "NDFit" Method. MINOS Internal Document 1538.

[101] S. Kopp, Z. Pavlovich, and P. Vahle, Effect of Beam Uncertainties on NuMI Analyses, Presentation at NuFact 06, UC Irvine, Aug 24-30, 2006.

[102] Rebel, B. MINOS Internal Document 2884.

[103] Petyt, D. MINOS Internal Document 3179.

[104] Feldman, G. "MINOS Blind Analysis Policy" MINOS Internal Document 1093.

[105] Litchfield, P. "Far Detector event preselection (data cleaning) for NC analysis" MINOS Internal Document 3377.

[106] Blake, A. "Far Detector Data Quality Software" MINOS Internal Document 3486.

[107] Press, W. H. et al. "Numerical Recipes in C++", Cambridge, UK: Univ. Pr. (1992) 994p.

[108] Hallagher, H., et al. Event Generator Uncertainties and the $1 \mathrm{e} 20 \mathrm{POT} \nu_{\mu} \mathrm{CC}$ Analysis. MINOS Internal Doc 1288

[109] Estimated Uncertainty on the Shower Energy Scale in Muon Neutrion CC Events, MINOS Internal Document 3362.

[110] Tracking Efficiency 'position paper', MINOS Internal Document 3356. Range Curvature Task Force Position Paper, MINOS Internal Document 3134.

[111] A Data-Driven Correction to the MC NC Background, MINOS Internal Document 3307. 
[112] Nelson, J. The density of MINOS Steel, MINOS Internal Document 1431.

[113] Osiecki, T. CC and Low Completeness Background in the NC Spectrum, MINOS Internal Document 2244.

[114] Osiecki, T. Marquardt Style NDFit For an $f_{\text {sterile }}$ Measurement in MINOS. MINOS Internal Document 3166.

[115] Kordosky, M. Effect of Intranule/Hadronization Simulations on Energy Carried by Showers. MINOS Internal Document 3152.

[116] Petyt, D. Far Detector Data Quality Checks. MINOS Internal Document 2903.

[117] Vahle, P. Beam Systematics Update. MINOS Internal Document 2941. 


\section{Vita}

Thomas Henry Osiecki was born on August 14, 1980 in Chicago, Illinois to Leszek and Genowefa Osiecki. After graduating as Salutatorian from Lemont Township High School he attended the University of Chicago. At Chicago he graduated with a Bachelor of Arts in physics and a Bachelor of Science in mathematics with specialization in computer science in the spring of 2002. Immediately after graduation, he entered the physics department at the University of Texas at Austin for graduate studies. His entire graduate career has been spent working on the MINOS experiment which has included over a year's residence at Fermilab National Accelerator Laboratory. He currently resides in Austin, but will be moving soon to Los Angeles to start work in the aerospace industry.

Permanent Address: 4705 Depew Ave

Austin, TX 78751

This dissertation was typeset with $\mathrm{LT}_{\mathrm{E}} \mathrm{X} 2 \varepsilon^{1}$ by the author.

\footnotetext{
${ }^{1} \mathrm{~L}_{\mathrm{E}} \mathrm{X} 2 \varepsilon$ is an extension of $\mathrm{IAT}_{\mathrm{E}} \mathrm{X}$. $\mathrm{L}_{\mathrm{A}} \mathrm{T}_{\mathrm{E}} \mathrm{X}$ is a collection of macros for $\mathrm{T}_{\mathrm{E}} \mathrm{X}$. $\mathrm{T}_{\mathrm{E}} \mathrm{X}$ is a trademark of the American Mathematical Society. The macros used in formatting this dissertation were written by Dinesh Das, Department of Computer Sciences, The University of Texas at Austin, and extended by Bert Kay, James A. Bednar, and Ayman El-Khashab.
} 